\title{
Miniature Implantable Telemetry System for Pressure-Volume Cardiac Monitoring
}

Kyle G. Fricke, The University of Western Ontario

Supervisor: Dr. Robert Sobot, The University of Western Ontario

A thesis submitted in partial fulfillment of the requirements for the Doctor of Philosophy degree in Electrical and Computer Engineering

(C) Kyle G. Fricke 2016

Follow this and additional works at: https://ir.lib.uwo.ca/etd

Part of the Biomedical Devices and Instrumentation Commons

\section{Recommended Citation}

Fricke, Kyle G., "Miniature Implantable Telemetry System for Pressure-Volume Cardiac Monitoring" (2016). Electronic Thesis and Dissertation Repository. 4303.

https://ir.lib.uwo.ca/etd/4303

This Dissertation/Thesis is brought to you for free and open access by Scholarship@Western. It has been accepted for inclusion in Electronic Thesis and Dissertation Repository by an authorized administrator of Scholarship@Western. For more information, please contact wlswadmin@uwo.ca. 


\begin{abstract}
Safety Pharmacology is becoming an increasingly important field to pharmaceutical companies in order to determine if medical treatments (drugs) are safe for use in human subjects. In the pre-clinical phase, test models, most commonly small rodents, undergo various gene alterations to affect, for example, their cardiovascular system in order to emulate human diseases. Pharmaceutical companies rely on these models to determine the drug efficacy and to ensure minimal side effects and safety for human subjects. Currently, no fully implantable left-ventricle pressure-volume (PV) telemetry systems have been implemented for Safety Pharmacology assessment applications. A fully implantable PV telemetry system would not only allow safety pharmacologists to better determine a drugs effect on the heart over traditional assessment methods (heart rate, blood pressure, ECG) but would also improve drug efficacy with the additional physiological parameters that PV loops provide.

The research in this thesis focuses on the design, implementation, and testing phases of a wireless telemetry system for pressure-volume cardiac monitoring in a live animal subject. A commercial MEMS PV sensor is implanted into the subject's left-ventricle (LV), while the telemetry system is implanted outside of the heart and connected to the sensor with a 7microwires tether. The RF telemetry system is suitable for commercial application in medium sized subjects, its total volume of $2.475 \mathrm{~cm}^{3}$ and a weight of $4.0 \mathrm{~g}$. The designed system is $58 \%$ smaller in volume, $44 \%$ in weight and has a $55 \%$ reduction in sampling power over the last reported research in PV telemetry. In-vivo data was captured in both an acute and a freely moving setting over a 24 hour period.

This real-time implantable telemetry system allows researchers to continuously monitor cardiac health by collecting real-time chronic PV data from freely moving conscious animals. Thus, enabling safety pharmacologists to utilize the gold standard of PV loops in their drug studies by using PV implants, facilitating safer and more effective drugs for human use.
\end{abstract}

Keywords: mixed-signal, implantable, bio-medical applications, radio frequency, sensors, pressure-volume, low-power, admittance, lock-in amplifier, conductance based catheter, 
swine, cardiac, safety pharmacology, contractility, biomedical telemetry, implantable medical devices, blood pressure, blood volume, left-ventricle 


\section{Acknowledgements}

First and foremost, I would like to thank my supervisor Dr. Robert Sobot. I greatly appreciate his guidance and mentorship throughout my graduate career. As my professor, supervisor, and mentor he has provided me with invaluable knowledge both in engineering and life. His continued support and patience has allowed me to excel in graduate studies and obtain many great achievements.

I would like to express my thanks to my present and past colleagues in our implantable systems research group, Sneha Lele, Kyle De Gannes, Shawon Senjuti, Mengye Cai, Ziyu Wang, Naeeme Modir, Brett Poulsen, and Mike Zylstra for their care, assistance and fellowship. Furthermore, I appreciate the many undergraduate students that I had the opportunity to mentor: Sorin Popa, Gail Hayhoe, Ryan Maksymic, Elen Badel, Rangababu Ganta, and Joilen Van Gaalen. All of you have have made the process of graduate studies more enjoyable and I am happy to see everyone so successful in their carriers around the world. To my friends and colleagues who have supported me throughout the years, specifically Kevin Brightwell, Justin Szoke-Sieswerda, Michael Gverzdys, Joesph Donohue, and Steve Toth, I greatly value your friendship and support.

I am immensely thankful for the support, guidance, resources, and industry knowledge that the research and engineering team at Transonic Scisense Inc. provided throughout my graduate studies. More specifically, special thanks to Peter Plouf, Chad Hodgson, Kim Wood, Filip Konecny, and Morteza Araghi. I would like to express my gratitude to the surgical team at Western University Veterinary Services, specifically, Alexander El-Warrak, Tracy Hill, and Heather Cadieux-Pitre. Without their veterinary surgical expertise, this research would not have occurred.

I would like to acknowledge the Natural Sciences and Engineering Research Council of Canada (NSERC), Ontario Graduate Scholarship, Ontario Centres of Excellence, CMC Microsystems, CFI, our industrial sponsor Transonic Scisense Inc. and the Electrical and Computer Engineering department at Western University for providing the facilities and funding 
that made this research possible. To all my course instructors, staff, faculty, and electronics shop who have helped me along the way, your support does not go unnoticed. I am grateful to all the examiners who volunteered to provide me with useful and valuable feedback on my research.

I would like to thank my mother, father, and sister for everything they have done for my education and for always believing in me throughout my university career. Thanks to all my friends and relatives for always being helpful and encouraging during my studies. Finally, I would like to thank my wife, Jessica, who has been here with me throughout my whole university career. She supports me in anything that I do and has been a constant source of inspiration. I am eternally grateful for her love and support. 


\section{Table of Contents}

\begin{tabular}{lll}
\hline Abstract & i
\end{tabular}

\begin{tabular}{|ll}
\hline Acknowledgements & iii
\end{tabular}

\begin{tabular}{|l|l}
\hline List of Figures & ix
\end{tabular}

\begin{tabular}{ll}
\hline List of Tables & xvi
\end{tabular}

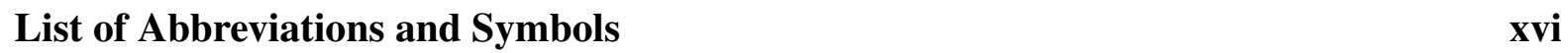

$\begin{array}{lll}1 & \text { Introduction } & 1\end{array}$

1.1 Motivation . . . . . . . . . . . . . . . . . . . . . . . . . . . . . . . . .

1.2 Research Problem . . . . . . . . . . . . . . . . . . . . . . . 99

1.3 Research Objectives . . . . . . . . . . . . . . . . . . . . . . . . . . . . . . . . . . . . . . . .

1.4 Organization of the Thesis $\ldots \ldots \ldots \ldots \ldots$

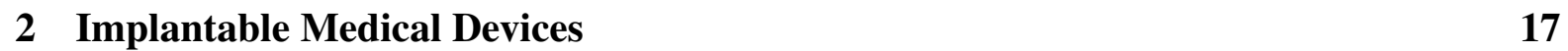

2.1 Overview of Implantable Medical Devices . . . . . . . . . . . . . . . . . . . 17

2.1.1 Implant Design Challenges and Concerns . . . . . . . . . . . . . . . 24

2.2 Blood Pressure . . . . . . . . . . . . . . . . . . . 27

2.2 .1 Blood Pressure Measurement Techniques in Animals . . . . . . . . . . 29

Indirect Measurement Methods . . . . . . . . . . . . . . . . . . . . 29

Direct Measurement Methods . . . . . . . . . . . . . . . . . . 30

2.3 Blood Volume Measurement in Animals . . . . . . . . . . . . . . . . . . . 39

2.3 .1 Conductance . . . . . . . . . . . . . . . . . . 39

2.3 .2 Admittance . . . . . . . . . . . . . . . . . . . 42

2.3 .3 Pressure-Volume Systems . . . . . . . . . . . . . . . 44

2.4 Conclusion $\ldots \ldots \ldots \ldots \ldots \ldots$

3 Numerical Comparison and Calibration of Conductance Catheter Models 50

3.1 Conductance Based PV Measurements . . . . . . . . . . . . . . 51

3.1.1 Review of Ideal Conductance-to-Volume Technique . . . . . . . . . . 51

3.1 .2 Conductance-to-Volume Equations . . . . . . . . . . . . . 53

3.1 .3 Calibration Techniques . . . . . . . . . . . . . . 55

3.2 Numerical Comparison of Equations . . . . . . . . . . . . . . . . 56

$3.2 .1 \quad$ FEM Numerical Conductance Model Setup . . . . . . . . . . . . . . . 56

3.2.2 Analytical Conductance-to-Volume Model Accuracy . . . . . . . . . . 57 
3.2.3 Comparison of Conductance-to-Volume Equations . . . . . . . . . . . 61

3.3 In Vitro Catheter Experiments . . . . . . . . . . . . . . . 64

$3.3 .1 \quad$ In-Vitro Conductance Catheter Experimental Setup . . . . . . . . . . . 64

3.3.2 In-Vitro Conductance Catheter Experimental Results . . . . . . . . . . 67

3.4 Catheter Geometric Sensitivity Analysis . . . . . . . . . . . . . . . . 70

$3.4 .1 \quad$ Catheter Sensitivity Geometric Setup . . . . . . . . . . . . . . . . . 71

3.4 .2 Sensitivity Simulation Results . . . . . . . . . . . . . . . . . . . . . . . . . 72

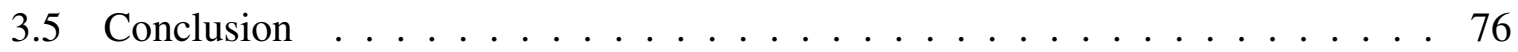

\begin{tabular}{|ll|}
\hline Wireless PV Telemetry System & 78
\end{tabular}

4.1 DC Power Module $\ldots \ldots \ldots \ldots$. . . . . . . . . . . . . . . . 79

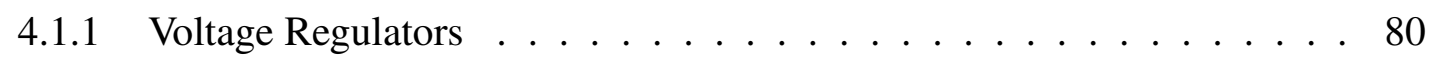

Microcontroller and Transceiver Power Supply . . . . . . . . . . . . 80

Interface Power Supply . . . . . . . . . . . . . . . . . . . . . . . . . . . . . . . . . .

Virtual Ground . . . . . . . . . . . . . . . . . . . . . 8 81

4.1 .2 Supervisor Device. . . . . . . . . . . . . . . . . . . . . . . . . . . . . . 82

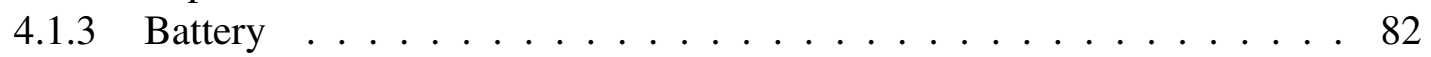

4.1 .4 Power Module Implementation . . . . . . . . . . . . . . . . . . . . . . . . . . . . . . 83

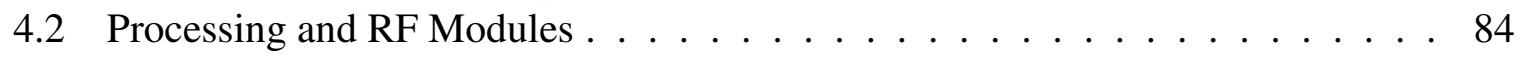

4.2 .1 Microcontroller Module . . . . . . . . . . . . . . . . . . . 84

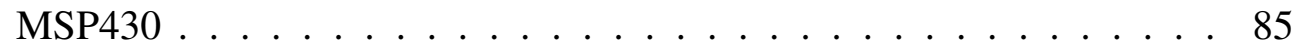

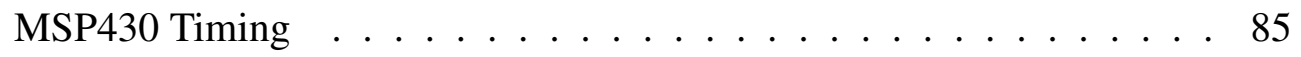

Analog to Digital Conversion in the MSP430 . . . . . . . . . . . . . . 86

Power Management . . . . . . . . . . . . . . . . . . . . . 87

Firmware ........................... . . . . . . . . . . . . 88

Microcontroller Module Implementation . . . . . . . . . . . . . . . . . 88

4.2 .2 Radio Module . . . . . . . . . . . . . . . . . . . . . . 89

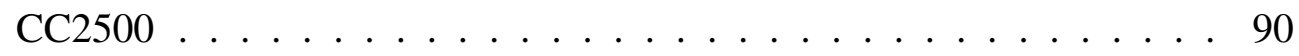

Communication Between Microcontroller and CC2500 . . . . . . . . . 91

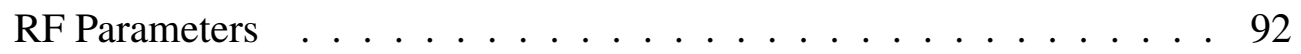

RF Module Implementation . . . . . . . . . . . . . . . . . . . . . . . 92

4.3 Communications Bus . . . . . . . . . . . . . . . . . . . 94

4.3 .1 Vertical Connectors - Physical Construction: . . . . . . . . . . . 96

4.4 PV Sensor Interface Module $\ldots \ldots \ldots 6$

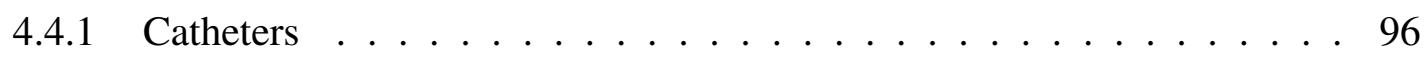

4.4 .2 Pressure Measurement . . . . . . . . . . . . . . . . . . . . . . 97

Traditional Wheatstone Bridge . . . . . . . . . . . . . . . . . 999

Digital Pressure IC . . . . . . . . . . . . . . . . . . . . . . . . . . . . . . . . . . . . . . . .

4.4 .3 Volume Measurements . . . . . . . . . . . . . . . . . . 104

Lock-In Amplifier Background Theory . . . . . . . . . . . . . . . . . 104

Lock-In Amplifier Circuit Implementation. . . . . . . . . . . . . . . . 110

4.4 .4 Interface Module - Implementation . . . . . . . . . . . . . . . . 116

4.5 Testing Platform . . . . . . . . . . . . . . . . . . . 118

4.5 .1 Breakout Board . . . . . . . . . . . . . . . . . . 118

4.5 .2 Development Board . . . . . . . . . . . . . . . 119 
Battery Charging . . . . . . . . . . . . . . . . . . . . . 119

Power Mangement - Development Board . . . . . . . . . . 120

Programming and Testing - Development Board . . . . . . . . . 120

4.6 Telemetry System Assembly Implementation $\ldots \ldots$. . . . . . . . . . . . . 121

4.7 Control Architecture and Current Consumption . . . . . . . . . . . . . . 123

4.7 .1 Implant Initialization $\ldots \ldots \ldots \ldots \ldots \ldots$

4.7 .2 Data Acquisition . . . . . . . . . . . . . . . . . 125

Continuous . . . . . . . . . . . . . . . . . 127

Duty Cycle . . . . . . . . . . . . . . . . . . . . 127

Sleep Cycle . . . . . . . . . . . . . . . . . . . . . . . . 127

4.7 .3 Current Consumption . . . . . . . . . . . . . . . . . 128

4.8 External Receiving Unit $\ldots \ldots \ldots \ldots$

4.8 .1 Wireless Receive Block . . . . . . . . . . . . . . . . . . 131

4.8 .2 UART Module $\ldots \ldots \ldots \ldots \ldots$

$4.8 .3 \quad \mathrm{PC} /$ External Unit . . . . . . . . . . . . . . . . . . . . . . . 134

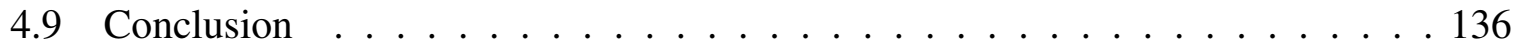

$\begin{array}{|lr|}5 \text { In-Vitro Characterization } & 137\end{array}$

5.1 Phantom Tissue Evaluation . . . . . . . . . . . . . . . . . . . 137

$5.1 .1 \quad$ Phantom Tissue Study Setup . . . . . . . . . . . . . . . . . . . 138

5.1 .2 EM Simulation Model $\ldots \ldots \ldots$. . . . . . . . . . . . . . . . 141

5.1 .3 Phantom Tissue Results. . . . . . . . . . . . . . . . . . . . . . . 142

5.2 Implant Package Design and Evaluation . . . . . . . . . . . . . . . . 147

$5.2 .1 \quad$ Biomedical Implant Capsule Design . . . . . . . . . . . . . . 147

5.2 .2 Implant Capsule Water Bath Test . . . . . . . . . . . . . . . . 151

Implant Capsule Preparation . . . . . . . . . . . . . . . . . . 151

Experimental Setup . . . . . . . . . . . . . . . . . . . 153

Water Bath Results . . . . . . . . . . . . . . . . . . . 156

5.2 .3 Enclosure for In-Vivo Study . . . . . . . . . . . . . . . . . 158

5.3 PV System Calibration . . . . . . . . . . . . . . . . . . . . . . . . 160

5.3 .1 Pressure Calibration. . . . . . . . . . . . . . . . 160

Electronic Setup . . . . . . . . . . . . . . . . . . 160

Pressure Calibration Chamber Setup . . . . . . . . . . . . . . 162

Pressure Calibration Process $\ldots \ldots \ldots$. . . . . . . . . . . 163

5.3 .2 Volume Calibration . . . . . . . . . . . . . . . . . . . . . . . 164

Volume Calibration Setup. . . . . . . . . . . . . . . . . . . . . . 164

Calibration Matrix . . . . . . . . . . . . . . . . . . . 164

Calibration Curves . . . . . . . . . . . . . . . . . 167

Calibration Methods Calculation Summary _. . . . . . . . . . . . 173

5.4 Dynamic PV Loop Evaluation $\ldots \ldots$. . . . . . . . . . . . . . . 175

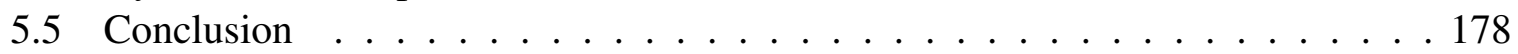

6 In-Vivo Implant Characterization 180

$6.1 \quad$ LabView Custom Measurement Application . . . . . . . . . . . . . . . 180

$6.1 .1 \quad$ Serial Port Setup $\ldots \ldots \ldots \ldots \ldots \ldots$ 
6.1 .2 Data Read . . . . . . . . . . . . . . . . . . . 183

6.1 .3 Remote Implant Data Write . . . . . . . . . . . . . . . . . . 186

6.1 .4 Limitations of Application V1 . . . . . . . . . . . . . . . . . . . . . . 189

6.2 In-Vivo Study . . . . . . . . . . . . . . . . . . . . . . . . . . . . . 189

6.2 .1 Experiment Overview . . . . . . . . . . . . . . . . . . . 190

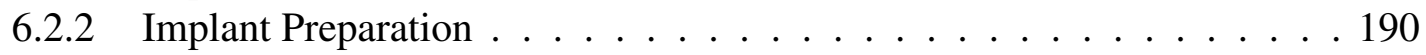

6.2 .3 Telemetry System Implantation Procedure . . . . . . . . . . . . . . . . 191

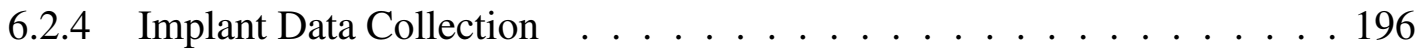

Acute Observation . . . . . . . . . . . . . . . . 196

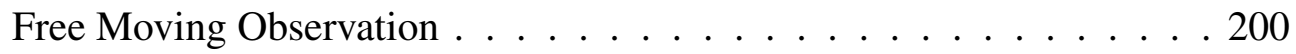

6.2 .5 Implant and Catheter Tissue Impact ～. . . . . . . . . . . . . . . . . 204

Macroscopic observation . . . . . . . . . . . . . . . . 204

Tissue sample preparation for microscopic evaluation: . . . . . . . . . 205

Histopathology Results . . . . . . . . . . . . . . . . . . . 208

6.2 .6 In-Vivo Study Discussion . . . . . . . . . . . . . . . . . 210

6.3 Conclusion . . . . . . . . . . . . . . . . . . 213

\begin{tabular}{lll}
\hline & Conclusion & 214
\end{tabular}

7.1 The Contribution of the Thesis . . . . . . . . . . . . . . . . . 214

7.2 Future Work . . . . . . . . . . . . . . . . . . . . . 219

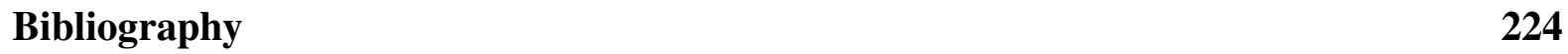

$\begin{array}{lr}\text { Curriculum Vitae } & 260\end{array}$ 


\section{List of Figures}

1.1 Diagram of a human heart, displaying the right and left atriums and ventricles. Licensed by CC BY-SA 3.0. . . . . . . . . . . . . . . . . . 2

1.2 Pressure vs Volume (PV) Loop. Stroke Volume (SV) is calculated by subtracting ES volume from ED volume. This is used to calculate the Cardiac Output (CO) in conjunction with the heart rate. A larger SV indicates healthier heart. . 3

1.3 Traditional Wiggers diagram that shows the relationships between Aortic pressures, left ventricle pressures, left ventricle volume, and ECG. [1,2] Licensed

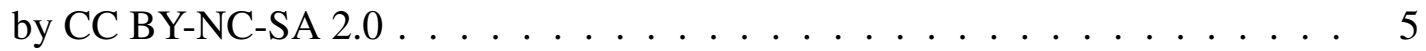

1.4 Commercial Pressure-Volume Tethered Measurement System from Transonic Scisense Inc. Interface Module, 1.2F Catheter, External Processing Unit [3]. . . 11

1.5 RF telemetry system shown inside a rabbit model. Note: implant location not

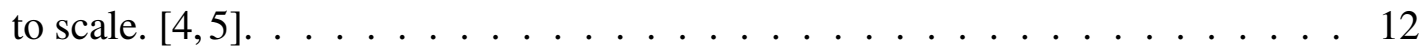

$1.6 \quad$ Wireless implantable system active cage measurement environment, [4] . . . . 13

2.1 Implantable Neural Monitoring System by [6] where a) the block diagram and PCB implementation; b) system on mounted on rat's head. Images from [6]. Copyright (c)2011 IEEE. . . . . . . . . . . . . . . . . . . . . . . . 19

2.2 Wireless headstage for combined optogenetics and multichannel electrophysiological recording by [7] where a) is the two-sided view of the rigid-flex system, b) top system view folded with battery, c) side view attached to the implantable electrode module. Images from [7]. Copyright (c)2016 IEEE. . . . . . . . . . . 20

2.3 Implantable neural recording system proposed by [8] where the SOC is located under the skin with a larger external device located on the head of the subject. Images from [8]. Copyright (c)2016 IEEE. . . . . . . . . . . . . . 20

2.4 Implantable system by [9] for spike-triggered microstimulation in rats. Images from [9]. Copyright (c)2011 IEEE. . . . . . . . . . . . . . . . . . 21

2.5 Artificial pancreas for treatment of diabetes from [10]. Images from [10]. Copyright (c)2014 IEEE. . . . . . . . . . . . . . . . . . . . . 22

2.6 Three versions of Baj-Rossi's fully implantable remote monitoring system [11]. Implant contains custom IC measuring and processing the molecule concentrations as well as wireless data and power circuits. Images from [11]. Copyright (c)2016 IEEE. . . . . . . . . . . . . . . . . . . 23

2.7 Implantable telemetry system for cardiac pacemaker applications designed in [12]. Images from [12]. Copyright (c)2011 IEEE. . . . . . . . . . . . . . . 23

2.8 Smart hip implant proposed by [13]. Images from [13]. Copyright (C)2016 IEEE. 24

$2.9 \quad$ First solid state based implantable pressure system in 1989 [14, 15]. . . . . . . 32 
2.10 MEMS based pressure catheters for small animals from two manufactures: a) Millar Inc. and b) Transonic Scisense. . . . . . . . . . . . . . . . . . 33

2.11 Millar TRM54P implantable pressure telemetry system [16]. Copyright (C)Millar Instruments Inc. . . . . . . . . . . . . . . . . . . . . . . 34

2.12 Transonic EndoGear for a) large animal EndoGear1 [17] and b) small animal EndoGear 3 [18]. Copyright (c)Transonic Inc. . . . . . . . . . . . . . . . . 35

2.13 DSI HD-S21 small animal left ventricle pressure measurement system [19]. Copyright (CDSI. . . . . . . . . . . . . . . . . 36

2.14 DSI L11 large animal left ventricle pressure measurement system [20]. Copy-

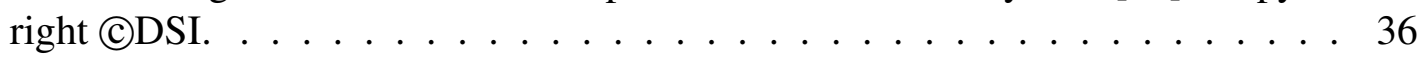

2.15 Implantable wireless telemetry system featuring a cuff blood pressure sensor: a) before packaging, b) after encapsulation, Cong [21]. Copyright (c)2010 IEEE. 37

2.16 Implantable cardiac monitoring system developed by Volk [22] incorporating ECG, arterial pressure, and temperature sensors. Copyright (c)2015 IEEE. .. . 38

2.17 Example of the physical size of a implantable catheter, along with the electrode spacing for volume measurements and pressure sensor location. . . . . . . . . . 39

2.18 Conductance $\left(G_{b}\right.$ and $\left.G_{m}\right)$ and Admittance $\left(G_{b}, G_{m}\right.$, and $\left.C_{m}\right)$ electrical heart model, where $I_{\text {in }}$ represents the injected current, $G_{b}$ blood conductance, $G_{m}$ muscle conductance, and $C_{m}$ as the capacitive component of the muscle. . . . . . 41

2.19 Implantable PV system developed by Raghavan [23] that is $17.67 \mathrm{~cm}^{2}$ and $27 \mathrm{~g}$. . 45

2.20 Implantable PV system developed by Schuhatovich [24] that weighs $4 \mathrm{~g}$ and has a volume of $10 \mathrm{~cm}^{3} . \ldots \ldots \ldots \ldots$. . . . . . . . . . . . . . .

2.21 Implantable PV system developed by Loeffler [25, 26] where a) shows the implant assembled with the battery; b) implant PCBs; and c) fully encapsulated implants. Implant is $5.92 \mathrm{~cm}^{3}$ in volume and has a weight of $7.2 \mathrm{~g} . \quad$. . . . . . . 47

2.22 System designed by Holt [27] to measure volume in the right ventricle. Volume

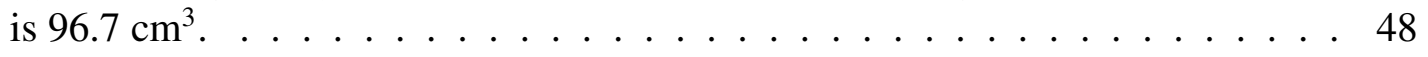

3.1 Geometry of a general conductance based measurement environment, where specific material properties can be modelled by $\sigma_{m}, \sigma_{b}, \epsilon_{r}, \epsilon_{m}$ and overall shape by R. Summary for eight catheter sizes used along with nominal $1.2 \mathrm{~F}$ is in

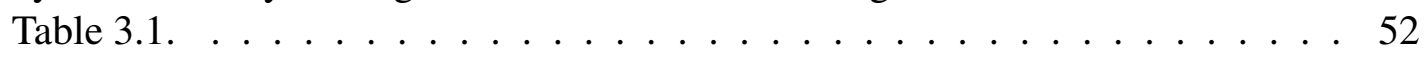

3.2 COMSOL FEM simulation of a standard geometry catheter in the standard

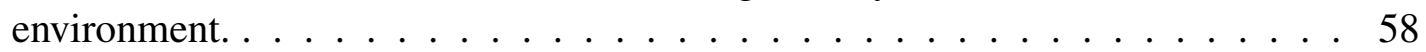

3.3 Conductance-volume data sets: a) Analytically calculated $G_{b}$ from known volumes (3.9), b) Simulated cylindrical electrodes, c) Spherical low conductance electrodes, d) Simulated spherical electrodes, e) Spherical low conductance point probes; are compared to analytical data for (3.9). . . . . . . . . . . . . 60

3.4 Comparison of calculated volumes from simulated data. . . . . . . . . . . . 62

3.5 Comparison conductance to volume equations vs known cylindrical volumes. Ideal relationship should be linear. . . . . . . . . . . . . . . . . 64

\begin{tabular}{ll}
3.6 & Radially organized cuvette tray with an integrated guide for various cylindrical \\
\hline & volume etalons. $\ldots \ldots \ldots \ldots \ldots$
\end{tabular}

$3.7 \quad$ Catheter 190 under microscope measuring source electrode rings. . . . . . . . . 66

3.8 Experimental and comparison methodology flow. . . . . . . . . . . . . 67 
3.9 Measured conductance vs known cuvette volumes for each catheter. Each data set also includes curve fit. . . . . . . . . . . . . . . . . . . . 68

3.10 Measured vs. simulated conductance for five catheters (volume only) used in

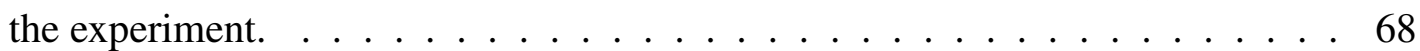

3.11 Measured Conductance for each different conductivity used vs measured conductance using nominal conductivity value of 0.77 . Conductivity values swept: $0.74,0.785,0.8,0.83$, and nominal $0.77 . \ldots \ldots \ldots$. . . . . . . . 69

3.12 COMSOL FEM simulation of a standard geometry catheter in an elliptical cu-

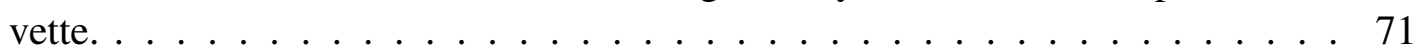

3.13 Root-mean square error versus catheter dimension $\mathrm{a} . \quad \ldots \ldots$. . . . . . . 73

3.14 Root-mean square error versus catheter dimension L. . . . . . . . . . . . 73

3.15 Root-mean square error versus catheter dimension a versus catheter dimension b versus catheter dimension L. . . . . . . . . . . . . . . . . . . . . . 74

3.16 Simulated calibration factor versus catheter dimension $\mathrm{d}$ and $\mathrm{L}$ for 27 simulated catheter geometries. . . . . . . . . . . . . . . . . . 75

3.17 Calibration factor versus catheter dimensions $d$ and L, five volume catheters only. 75

4.1 Block diagram of the wireless telemetry system environment consisting of the external side (PC, microcontroller, RF transceiver) and the implant (Power, RF transceiver, microcontroller, and analog front end) as well as the pressurevolume sensor. . . . . . . . . . . . . . . . . . . . . . 79

4.2 Full schematic of the Power Module. . . . . . . . . . . . . . . . 80

4.3 a) Dimensions of the battery used in the implantable system; b) Physical battery. 83

4.4 Power Module Physical Implementation. . . . . . . . . . . . . . . . . . 83

4.5 Microcontroller Module schematic. Input and output communication through

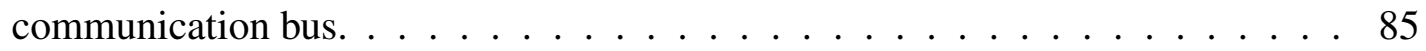

$4.6 \quad$ Microcontroller Module Physical Implementation. . . . . . . . . . . . . . . 88

$4.7 \quad$ Radio Module schematic with SPI communication through the bus. Matching network and RF antenna are also included. . . . . . . . . . . . . . . . . . 89

4.8 Frequency bandwidth of RF transceiver at a distance of $12 \mathrm{~cm}$, Tx output power $=-12 \mathrm{dBm} \mathrm{F}_{\text {Centre }}=2.433592 \mathrm{GHz} . \ldots \ldots \ldots \ldots \ldots$

$4.9 \quad$ SPI digital communication link between MSP430 and CC2500. . . . . . . . . 91

4.10 Radio Module: Left with external antenna, Right with PCB antenna. . . . . . . 93

4.11 Simplified block diagram of PV interface module, and the equivalent electrical diagram of MEMS pressure and conductance based volume sensors themselves; (in reality, ground line is shared). . . . . . . . . . . . . 97

4.12 Pressure Volume catheter a) inserted into the LV of a heart; b) physical implementation of the $5 \mathrm{~F}$ catheter; and c) dimensions of the $5 \mathrm{~F}$ catheter. $\ldots \ldots .98$

4.13 Traditional pressure measurement block. . . . . . . . . . . . . . . 99

4.14 Current source design. . . . . . . . . . . . . . . . . . . . . 100

4.15 ZDMI ZSC 31015 based Digital Pressure Measurement Method. . . . . . . . 102

4.16 ZDMI ZSC 31015 Internal Block Diagram [28]. Copyright (cZMDI. . . . . . . 103

4.17 Block diagram of a Lock-In Amplifier. . . . . . . . . . . . . . . . 105

4.18 Ideal mixer implementation. . . . . . . . . . . . . . . . . 106 
4.19 Phase Sensitive detector examples where the output $\mathrm{V}_{P S D}$ (black) is shown with $\mathrm{V}_{a c}(\mathrm{red})$ and $\mathrm{V}_{\text {ref }}\left(\right.$ blue) at different phase shifts: a) in phase; b) $180^{\circ}$; c) $90^{\circ}$; and d) $60^{\circ}$. The output of the low pass filter is not shown. . . . . . . . . . 109

4.20 Dual Lock-In Amplifier block diagram. . . . . . . . . . . . . . . . . . . . 110

4.21 Block Diagram of Volume Measurement Processing. . . . . . . . . . . . . . . 111

4.22 Quadrature Oscillator (outputs sine and cosine signals) designed with a $\mathrm{F}_{0}=20$

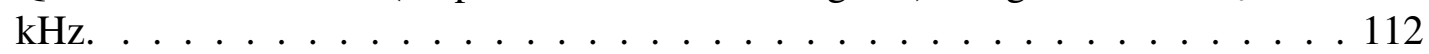

4.23 Voltage to Current converter implemented. . . . . . . . . . . . . . . 113

4.24 Instrumentation Amplifier and Gain Stage configuration. . . . . . . . . . . . . 114

4.25 ECG bandpass filter to remove ECG signals measured from surrounding heart

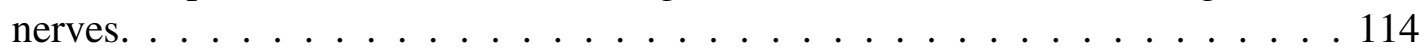

4.26 Inverter and analog switch, represents the phase sensitive detector in the lock-in

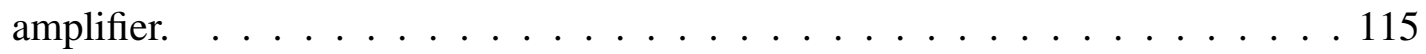

$4.27127 \mathrm{~Hz}$ Low Pass filter with gain and span included. . . . . . . . . . . . . . . . 116

4.28 Interface Module - Pressure and Volume PCB: Top PCB, left, and right inner PCB. . . . . . . . . . . . . . . . . . 117

4.29 (a) Breakout board and (b) Development boards of the second physical prototype. 119

4.30 Development and Breakout Module connected via flex cables. . . . . . . . . 120

4.31 Close up 3D rendering of stacked vertical connectors (left), and exploded view of the telemetry system with 5 PCBs from the PV sensor interface at the top, to the power supply module at the bottom (right). . . . . . . . . . . . . 122

4.32 Full 3D rendering of assembled wireless telemetry system with battery (left), and photograph of fully assembled prototype (without battery) next to a Canadian dime (right). . . . . . . . . . . . . . . . . . 123

4.33 Control Architecture flow of timer interrupt (Data Acquisition) and main CPU

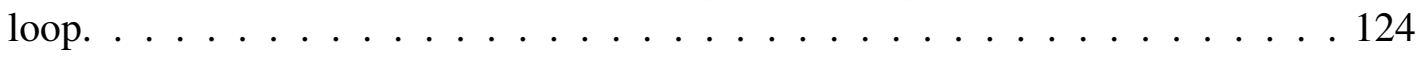

4.34 Implant initialization: Board Initialization, Calibration of VLO, and Transceiver

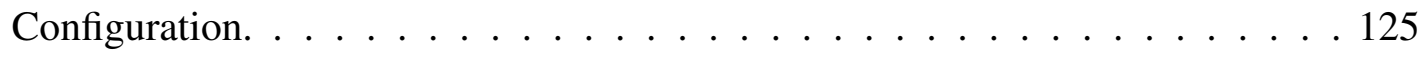

4.35 Instantaneous current and power consumption of the full telemetry system during a single data acquisition and transmission cycle through air with a load of $R_{\mathrm{L}}=100 \Omega$ and output power of $-12 \mathrm{dBm}$. Total time per acquisition: Sampling

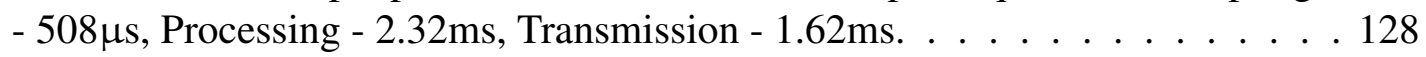

4.36 RX Unit Block Diagram that includes: the Wireless Receive Block, UART Module, and PC. . . . . . . . . . . . . . . . . . . . 130

4.37 Wireless receive block including MSP430 programmer (right) and ez430-RF2500 (left) [29]. Copyright (c)Texas Instruments. . . . . . . . . . . . . . . . 131

4.38 UART Hardware Module Block Diagram that includes the USB connector to send the PC data, FTDI IC, and Program connector to interface with the MSP430.133

4.39 UART Hardware Module PCB. . . . . . . . . . . . . . . . . . . . . . . . . . . . . . . . . . . . . . . . .

4.40 Raw Data Streamed to PC via Putty. . . . . . . . . . . . . . . . . . 135

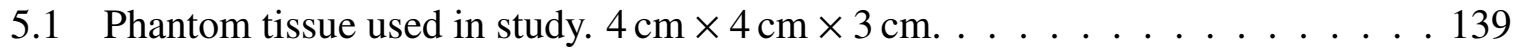

5.2 Implant antenna inserted into phantom tissue. . . . . . . . . . . . . . . 140 
5.3 Experimental Measurement Environment used in study. Shown: Spectrum analyzer, $\mathrm{R}_{x}$ antenna, $\mathrm{PV}$ prototype with antenna inserted into $4 \mathrm{~cm}$ phantom tissue $10 \mathrm{~cm}$ away. . . . . . . . . . . . . . . . . . 140

5.4 Designed $2.4 \mathrm{GHz}$ monopole antenna used in EM simulations. Left: Electric Field Distribution with 0dBm input power. . . . . . . . . . . . . . . . . 141

5.5 VSWR of 3D simulated antenna. . . . . . . . . . . . . . . . . . . 142

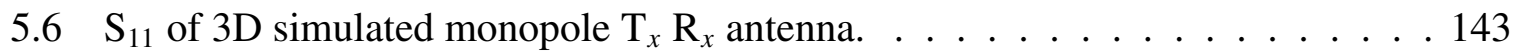

$5.7 \quad \mathrm{~S}_{21}$ of 3D simulated phantom tissue environment at a distance of $20 \mathrm{~cm}$ with $\mathrm{T}_{x}$ antenna inserted into phantom tissue model. . . . . . . . . . . . . . 143

5.8 Calculation of absorption losses of phantom tissue. . . . . . . . . . . . . . . 144

5.9 Comparison of different RF output power levels with and without the phantom tissue inserted. Without phantom tissue: (a) 0dBm, (b) $-12 \mathrm{dBm}$, (c) $-16 \mathrm{dBm}$, (d) -20dBm; With Phantom Tissue: (e) 0dBm, (f) -12dBm, (g) -16dBm, (h) $-20 \mathrm{dBm} \ldots \ldots \ldots \ldots \ldots \ldots \ldots \ldots$

5.10 Receive power with and without phantom tissue for output $\mathrm{T}_{x}$ power level of $-12 \mathrm{dBm}$. (a) $P_{\text {in }}-$ Incident Power, (b) $P_{r}-$ Power received without tissue, (c) $P_{\text {rt }}-$ Power received with phantom tissue, (d) $P_{\text {loss }}-$ Power loss without phantom tissue, (e) $P_{\text {losst }}-$ Power loss with phantom tissue, (f) $P_{\text {absorb-sim }}-$ Power absorbed by tissue simulation, $(\mathrm{g}) P_{\text {absorb-exp }}-$ Power absorbed by tissue

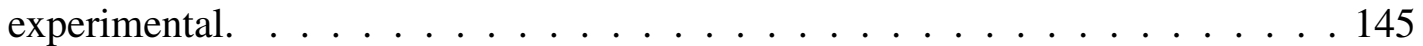

5.11 3D Printed Plastic Packages for Pressure-Volume Implant from left to right: PV Telemetry system [30, 31], thin wall acrylic, thick wall acrylic, thick wall ABS-M30i (ABS not shown). . . . . . . . . . . . . . . . . . . 148

5.12 3D Printed metal packages for pressure-volume implant: thin wall 316L stainless steel top (left) and bottom (right). . . . . . . . . . . . . . . . . . . . 149

5.13 Examples of errors when 3D printing the thin wall version in plastic. . . . . . 150

5.14 Implant package cross-section view: Thin wall package with $24 \mathrm{~mm}$ by $18 \mathrm{~mm}$ outer dimensions (left); and thick wall package with $32 \mathrm{~mm}$ by $20 \mathrm{~mm}$ outer dimensions (right). Outer wall thickness noted by hatched lines. . . . . . . . . 150

5.15 Experimental test setup - six liter water bath set to $(40 \pm 0.2)^{\circ} \mathrm{C}$, implants are submersed into water and held there for the duration of the study. . . . . . . . . 152

5.16 (a) Chlorophenol water test strips placed inside top and bottom cavities of implant capsule. The paper turns purple when in contact with water. (b) Fully sealed implant capsules with Loctite 4311 epoxy and Loctite 5091 silicone. . . 154

5.17 A closeup of the thick walled capsule 3D printed in RDG720 transparent acrylic material displaying internal chlorophenol water strips. . . . . . . . . . . . . . . 154

5.18 Internal view of water bath setup with each implant weighted down to prevent

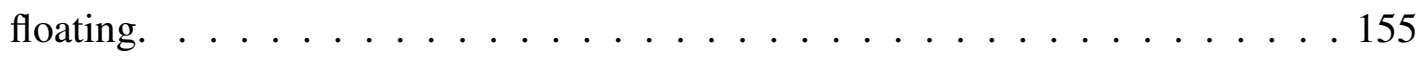

5.19 Water Bath Study Detection Paper Results: from left to right : Stainless Steel 316L, RDG720 Transparent Acrylic, ABS-M30i, ABS. Water moisture located in corner of ABS-M30i package. . . . . . . . . . . . . . . . 157

5.20 Stainless Steel 316L reaction locations at the catheter port hole and along the

5.21 a) 3D rendering of the final package design with internal PCB boards, catheter and suture holes shown; b) 3D printed prototype prior to surgery. . . . . . . . . 159 
5.22 Pressure calibration experimental setup. Shown: PV system, Development Module / Breakout board, PC, Software. Not shown: ZMDI Communication

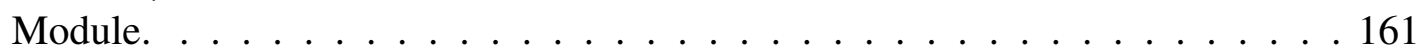

5.23 ZMDI Communication Module connection to Development module and PV

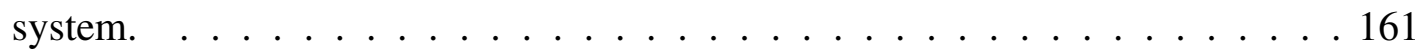

5.24 ZMDI evaluation software kit software [28]. Copyright CCZMDI. . . . . . . . . 162

5.25 Pressure chamber used in pressure calibration. . . . . . . . . . . . . . 163

5.26 Ideal $|Z|$ vs a) $|Z|_{\text {Calc }}$; b) $|Z|_{\text {Cal }}$ Method 1; c) $|Z|_{\text {cal }}$ Method 2. The trend line is also shown. . . . . . . . . . . . . . . . . 166

5.27 Ideal $\phi_{Z}$ vs a) $\phi_{\text {Calc }}$; b) $\phi_{\mathrm{ZCal}}$ Method 1; c) $\phi_{\mathrm{ZCal}}$ Method 2. The trend line is also shown. . . . . . . . . . . . . . . . . . 167

5.28 Ideal Load Resistance vs Ideal Load Capacitance vs System Phase Shift $\phi_{\text {System. }} 168$

5.29 Calculated Resistance $\mathrm{R}_{\text {Calc }}$ vs Calculated Capacitance $\mathrm{C}_{\text {Calc }}$ vs System Phase Shift $\phi_{\text {system. }} \ldots \ldots \ldots \ldots$. . . . . . . . . . . . . . . . . 169

5.30 Calculated Resistance $\mathrm{R}_{\text {Calc }}$ vs Calculated Capacitance $\mathrm{C}_{\text {Calc }}$ vs $|V|_{\text {Difference. }}$. . 170

5.31 Measured Voltage Magnitude $|V|_{\text {Measured }}$ Vs Measured Voltage Phase $\phi_{\text {Measured }}$

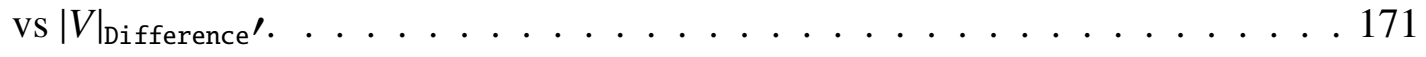

5.32 Measured Voltage Magnitude $|V|_{\text {Measured }}$ vs Measured Voltage Phase $\phi_{\text {Measured }}$

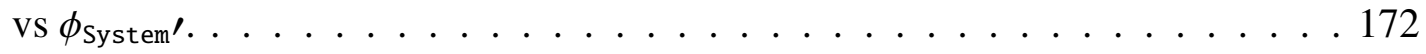

5.33 Volume Pulser with PV implant, Catheter, PV chamber, Labview interface shown. 176

5.34 Measured battery life with a duty cycle of 2 min on and 2 min with various $\mathrm{Rx}$

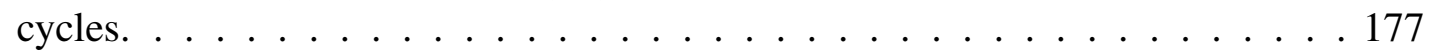

5.35 Battery life of implant in an extended duty cycle. . . . . . . . . . . . 178

6.1 Custom application front end: Receive data interface. . . . . . . . . . . . . . 181

6.2 Custom application front end: Send data interface. Application can change Tx power, acquisition modes, receive and duty cycle times using a single byte transmission. . . . . . . . . . . . . . . . . 182

6.3 LabView application block diagram including sub-blocks: Rx Unit, serial config, read, split string, convert to voltage, convert to $|Z|_{\text {Meas }}$ and $\phi_{\text {Meas }}$, calibrate pressure, and XY plot. . . . . . . . . . . . . . . . . 184

6.4 Combinational logic used such Rx changes take precedence over Duty cycle

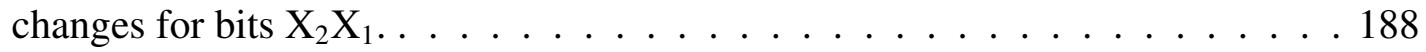

6.5 Seven sensor wires fed through the catheter into the enclosure attached to the telemetry system. . . . . . . . . . . . . . . . . 191

6.6 PV telemetry system fully enclosed in ABS-M30i package and dipped in silicone. Package used for transport. . . . . . . . . . . . . . . . . . . . 192

6.7 Fluoroscope image of 5F PV catheter inserted in the left ventricle. . . . . . . . 193

6.8 PV system implanted in neck region of test subject PV catheter inserted into LV via carotid artery. . . . . . . . . . . . . . . . . . . 194

6.9 Fluoroscope image of wireless pressure-volume system implanted inside swine

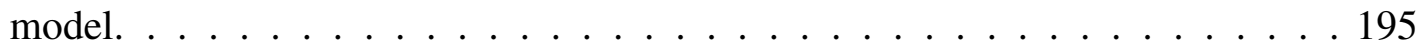

6.10 A sample of data collection during acute measurement phase where a) LV pressure; b) LV Volume; and c) PV loop. . . . . . . . . . . . . . . . . . . . . . . . 198 
6.11 Live data collection of left-ventricle pressure-volume signals from a freely moving swine model. . . . . . . . . . . . . . . 201

6.12 A sample of 12 seconds of data collection during free movement where a) LV pressure; b) LV Volume; and c) PV loop. . . . . . . . . . . . . . . . . . . . . 202

6.13 A four second sample demonstrating a) distorted PV loops; b) LV pressure. . . 203

6.14 Initial implant explant. Tissue growth observed on catheter coil. . . . . . . . . 205

6.15 Explanted implant, heart and portions of the carotid artery. Close-up of implant and tissue growth is shown. . . . . . . . . . . . . . 206

6.16 Dissection of left ventricle displaying broken catheter tip and aortic valve. . . . 207

6.17 Carotid artery with clotted blood. Tissue sample used in microscopic evaluation. 207

6.18 An example of Left ventricle: a) ulceration and hemorrhage with b) early granulation tissue as noted by a pathologist. . . . . . . . . . . . . . . 208

6.19 An example of Aortic Valve with ulveration and hemorrhage with early granulation tissue. Issues of concern as noted by a pathologist: (a) fibrin, (b) activated fibroblast, and c) hemosiderin. . . . . . . . . . . . . . . . . 209

6.20 An example of Carotid Artery with ulceration and hemorrhage, necrosis of intima, disruption and inflammation of the tunica adventitia as noted by a pathol-

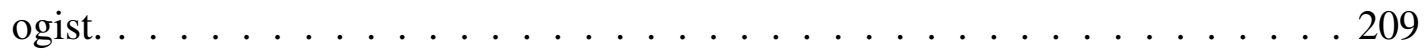

7.1 Improved version of PV telemetry system designed on a single PCB optimized for long-term studies. . . . . . . . . . . . . . . . . 220

7.2 Improve LabView measurement application interface. . . . . . . . . . . . . . 220

7.3 Custom test IC with Wireless power sub-blocks and ADC test blocks. . . . . . 222 


\section{List of Tables}

$1.1 \quad$ Various cardiac parameters $\ldots \ldots \ldots \ldots \ldots \ldots$

3.1 Physical dimensions of used catheters. . . . . . . . . . . . . . . 52

3.2 Data sets used to generate Fig. 3.3 Conductance-volume data sets: a) Analytically calculated $G_{b}$ from known volumes (3.9), b) Simulated cylindrical electrodes, c) Spherical low conductance electrodes, d) Simulated spherical electrodes, e) Spherical low conductance point probes; are compared to analytical data for (3.9). . . . . . . . . . . . . . . . . 60 60

3.3 Comparison between known cuvette volumes (simulated) and volumes calculated using (3.1), (3.4), and (3.8). Graphical representation Fig. 3.4] . . . . . . 63

3.4 Average Error of Wei's equation vs known volumes for each catheter when

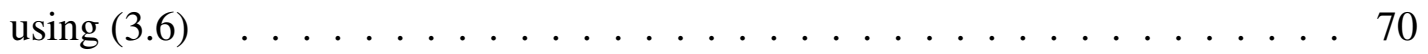

3.5 Fitting curve coefficients. . . . . . . . . . . . 76

4.1 TI MSP430 Low Power Modes and Clock Status . . . . . . . . . . . . . . . . 87

4.2 Communication Bus in the second prototype . . . . . . . . . . . . . . . 95

$4.3 \quad$ Average Current Consumption and Battery Life . . . . . . . . . . . . . . . . . 130

5.1 Phantom Tissue Ingredients [32] . . . . . . . . . . . . . . . . . . 138

5.2 Power consumption of Transmitter (CC2500) at various $T_{x}$ output powers, $\mathrm{VDD}=3.6 \mathrm{~V} \ldots \ldots \ldots \ldots \ldots \ldots$

5.3 Summary of 3D capsules printed . . . . . . . . . . . . . . . . 151

5.4 In-Vivo Calibration Coefficients for PV Volume Interface. . . . . . . . . . . 166

5.5 In-Vivo Calibration Data for PV Volume Interface. . . . . . . . . . . . . . 166

$6.1 \quad$ Rx to Implant Packet Format . . . . . . . . . . . . . . . . . . . . . . . . 187

6.2 Combinational Logic Truth Table . . . . . . . . . . . . . . . . . . . . . 188

6.3 Duty Cycle and Rx On and Off times used in in-vivo experiment . . . . . . . . 188

6.4 Physiological and Physical Paramaters used in Volume Conversion Using Wei's

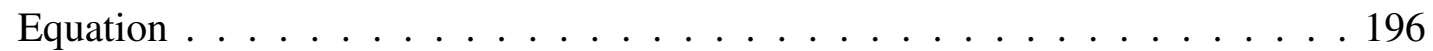

6.5 Extracted Physiological Data From PV Loops ～. . . . . . . . . . . . . . . . . 197

6.6 Comparison of Physiological Paramaters with those from [33] . . . . . . . . . 199

6.7 Comparison of relevant works f . . . . . . . . . . . . . . 212 


\section{List of Abbreviations, Symbols, and Nomenclature}

$\begin{aligned} \text { ABS } & \text { Acrylonitrile Butadiene Styrene } \\ \text { ABS - M30i } & \text { Stronger Bio-Compatible Version of ABS } \\ \text { AC } & \text { Alternating Current } \\ \text { ADC } & \text { Analog-to-Digital Converter } \\ \text { AFE } & \text { Analog Front End } \\ \text { Ah } & \text { Ampere Hour } \\ \text { BALUN } & \text { Balanced-Unbalanced } \\ \text { BNC } & \text { Bayonet Neill-Concelman Connector } \\ \text { CAD } & \text { Computer Aided Design } \\ \text { CMOS } & \text { Complementary Metal Oxide Semiconductor } \\ \text { CO } & \text { Cardiac Output } \\ \text { CPU } & \text { Center Processing Unit } \\ \text { CT } & \text { Computerized Tomography } \\ \text { dB } & \text { Decibel } \\ \text { DC } & \text { Direct Current } \\ \text { DCO } & \text { Digital Controlled Oscillator } \\ \text { DSP } & \text { Digital Signal Processing } \\ \text { ECG } & \text { Electrocardiogram } \\ \text { ED } & \text { End Diastolic } \\ \text { EDP } & \text { End Diastolic Pressure } \\ \text { EDV } & \text { End Diastolic Volume } \\ \text { EEG } & \text { Electroencephalogram } \\ \text { EF } & \text { Ejection Fraction } \\ \text { EMG } & \text { Electromyography } \\ \text { ES } & \text { End Systolic } \\ \text { ESP } & \text { End Systolic Pressure } \\ \text { ESV } & \text { End Systolic Volume } \\ \text { FCC } & \text { Federal Communications Commission } \\ \text { FDA } & \text { Food and Drug Administration, USA } \\ \text { FFC } & \text { Flat Flex Cable } \\ \text { FSK } & \text { Frequency Shift Keying } \\ \text { GPIO } & \text { General Digital Input and Output } \\ \text { HF } & \text { High Frequency } \\ & \end{aligned}$




$\begin{aligned} \text { IA } & \text { Instrumentation Amplfier } \\ \text { IC } & \text { Integrated Circuit } \\ \text { IDE } & \text { Integrated Development Environment } \\ \text { IEEE } & \text { Institute of Electrical and Electronics Engineers } \\ \text { IMD } & \text { Implanted Medical Device } \\ \text { Iq } & \text { Current Consumption } \\ \text { ISM } & \text { Industrial, Scientific and Medical } \\ \text { LDO } & \text { Low Drop Out } \\ \text { LED } & \text { Light Emitting Diode } \\ \text { LPF } & \text { Low Pass Filter } \\ \text { LPM } & \text { Low-Power Mode } \\ \text { LV } & \text { Left Ventricle } \\ \text { MEMS } & \text { Micro-Electro-Mechanical System } \\ \text { MICS } & \text { Medical Implant Communication Service } \\ \text { MRI } & \text { Magnetic Resonance Imaging } \\ \text { OP-Amp } & \text { Operational Amplifier } \\ \text { PCB } & \text { Printed Circuit Board } \\ \text { POT } & \text { Potentiometer } \\ \text { PSD } & \text { Phase Sensitive Detector } \\ \text { PSSR } & \text { Power Supply Rejection Ratio } \\ \text { PV } & \text { Pressure - Volume } \\ \text { RDG720 } & \text { Transparent Plastic from Stratasys Inc. } \\ \text { RF } & \text { Radio Frequency } \\ \text { RMS } & \text { Root Mean Square } \\ \text { RV } & \text { Right Ventricle } \\ \text { SMA } & \text { Low-Carbon Version of 316 Stainless Steel } \\ \text { SMA } & \text { Sub Miniature Connector Version A } \\ \text { SNR } & \text { Signal to Noise Ratio } \\ \text { SoC } & \text { System on Chip } \\ \text { SPI } & \text { Serial Peripheral Interface } \\ \text { SPICE } & \text { Simulation Program with Integrated Circuit Emphasis } \\ \text { SPST } & \text { Single Pole Single Throw } \\ \text { SRD } & \text { Short Range Device } \\ \text { SV } & \text { Stroke Volume } \\ \text { SW } & \text { Stroke Work } \\ \text { UART } & \text { Texas Instruments } \\ \text { USB } & \text { Universal Asynchronous Receiver/Transmitter } \\ \text { VCO } & \text { Voltage Controlled Oscillator } \\ \text { WPT } & \text { Wireless Power Transmission } \\ \text { XOR } & \text { Exclusive OR } \\ & \end{aligned}$




\section{Chapter 1}

\section{Introduction}

This chapter introduces the motivation behind this thesis. The research problem is outlined and solutions are explored. This is followed by research objectives and organization of the thesis.

\subsection{Motivation}

Safety Pharmacology is becoming an increasingly important field to pharmaceutical companies in order to determine if medical treatments are safe for use in human subjects [34, 35]. More specifically, in the cardiac field, Safety Pharmacology refers to the drug's (medical treatment) effects on the cardiovascular system [35]. It is extremely important for all parties involved (drug developer, clinical trial patient, end user, etc) for medical drugs to be safe and effective. Unsafe drugs that get released to the general population can cause adverse side-effects to patients and worse can cause death [36]. Pharmaceutical companies have a vested financial and ethical interest in the safety of the designed drug, as they have the possibility of gaining a high return on investment and novelty if found safe and effective, on the contrary they face consequential financial loss or company closure if unsafe. In the pre-clinical phase, the test models, most commonly small rodents, undergo various gene alterations to affect, for example, their cardiovascular system in order to emulate human cardiovascular diseases. Pharmaceutical companies rely on these models to determine the drug efficacy and to ensure minimal side 


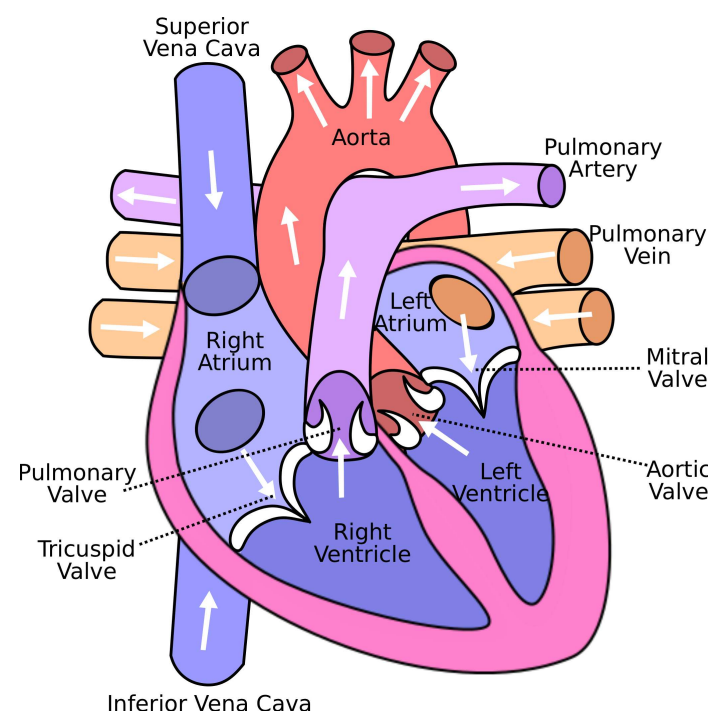

Figure 1.1: Diagram of a human heart, displaying the right and left atriums and ventricles. Licensed by CC BY-SA 3.0.

effects and safety for human subjects. Furthermore, the insights gained in Safety Pharmacology during clinical trials can greatly impact medical treatments for human diseases including cardiovascular disease and heart failure [37, 38].

The main organ in the cardiovascular system is the heart, as it regulates and pumps blood throughout the living body. The heart contains four chambers, two atria and two ventricles (left and right), Fig. 1.1. The left ventricle (LV) is generally studied over the right ventricle because it supplies blood to the whole body rather than just the lungs, as the right ventricle does, therefore accomplishes the majority of work in the heart [39]. In addition, the left ventricle pumps blood at a much higher pressures than the right ventricle. Two measurements, blood pressure and volume, of the LV provide insight into the efficiency of the living heart. Combining the measurement of the LV volume with measured LV pressure, pressure-volume (PV) loops can be created, Fig. 1.2, allowing for the extraction of various medical parameters.

A pressure-volume loop can be described as the PV relationship during a cardiac cycle. Here, the four stages of the cardiac cycle: diastolic filling, contraction, ejection (systolic phase), relaxation are represented by various blood pressure and volume values [40] [41], Fig. 1.2. A 


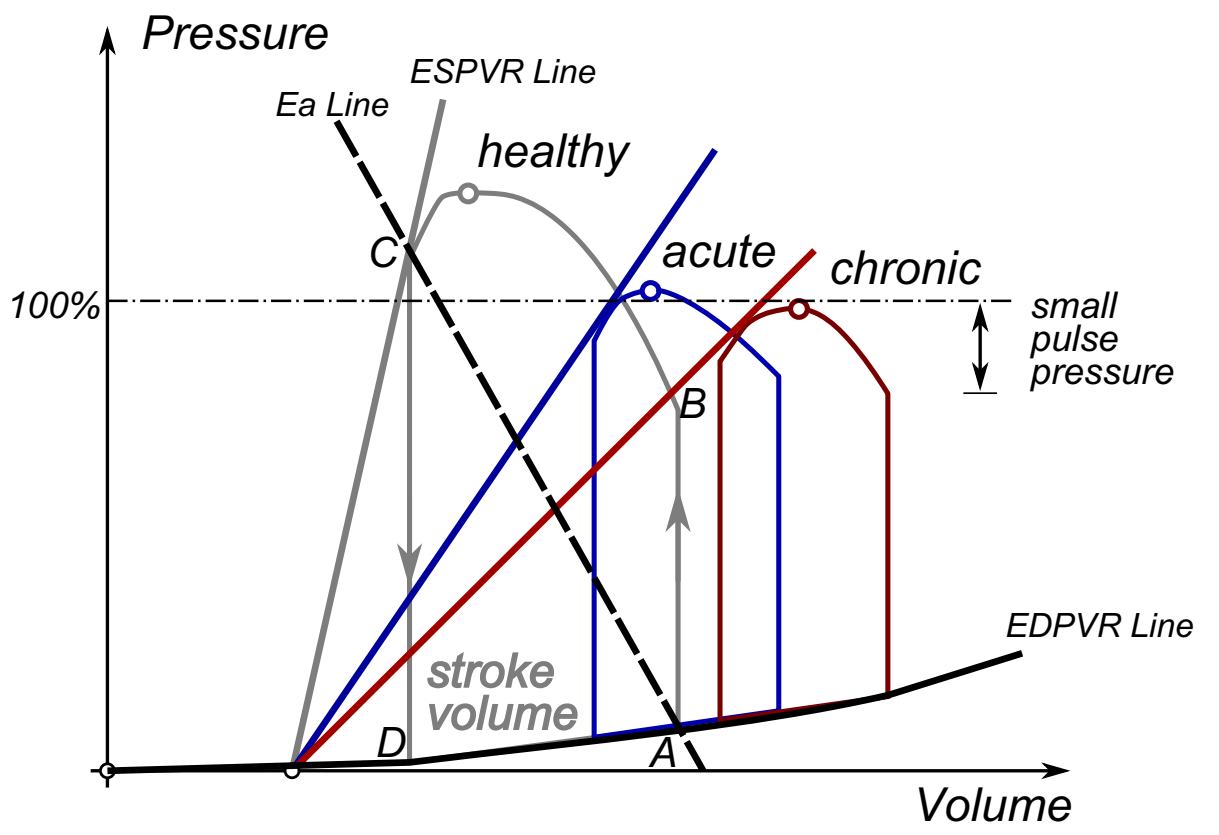

Figure 1.2: Pressure vs Volume (PV) Loop. Stroke Volume (SV) is calculated by subtracting ES volume from ED volume. This is used to calculate the Cardiac Output (CO) in conjunction with the heart rate. A larger SV indicates healthier heart.

brief overview of these four stages is given below:

- Stage 1: Diastolic Filling (between point D and A in Fig. 1.2): During this stage the LV muscles relax to allow for the increase in blood volume. The blood pressure during this stage is relatively low and constant and the blood volume increases to its highest level.

- Stage 2: Contraction (between point A and B in Fig. 1.2): This stage begins when the LV blood filling is complete. The mitral valve closes and the cardiac muscle starts to contract to increase blood pressure inside the LV. The blood volume during this stage is constant at its highest value (as both valves mitral and aortic are closed) and the blood pressure is constantly increasing. At the beginning of this stage, two important parameters are measured, A in Fig. 1.2, end-diastolic volume (EDV) and end-diastolic pressure (EDP). EDV and EPV represent the value of blood volume and pressure in the heart at the time filling is complete [40].

- Stage 3: Ejection (Systolic Phase): This stage begins at B and ends at C in Fig. 1.2, where 
the aortic valve opens up and the blood is ejected into the Aorta. This occurs as the left ventricular pressure is greater than the aortic pressure, thus causing the aortic valve to open. The heart muscles continue to contract to get the maximum blood ejection. The blood volume reduces to its minimum value during this stage

- Stage 4: Relaxation: The start of stage four ends the Systolic phase and the heart muscles begin to relax (i.e., reduce blood pressure) to allow the LV to refill and repeat the cycle (between point $\mathrm{C}$ and $\mathrm{D}$ in Fig. 1.2). At point $\mathrm{C}$, the aortic valve closes as aortic pressure is greater than LV pressure. This in conjunction with the heart relaxing causes the LV pressure to drop quickly. Note from $\mathrm{C}$ to $\mathrm{D}$, the mitral valve is still closed (i.e, no filling) just pressure reduction. At the beginning of this stage, two important parameters are measured, C in Fig. 1.2, end-systolic volume (ESV) and end-systolic pressure (ESP). ESV and ESP represent the value of blood volume and pressure in the heart at the time ejection is complete or at end of systole [40]. During this stage the blood volume is at its lowest and the pressure is reduced to its minimum. At the end of this stage, Point D, the LV pressure is below left atrium pressure which causes the mitral valve to open, thus causing diastolic filling and repeating the heart cycle.

The relationship between aortic pressure, LV pressure, LV blood volume, and the Electrocardiogram (ECG) is commonly described in the traditional Wiggers diagram, Fig. 1.3. This describes each major physiological signal in the time domain as it relates to the heart cycle. A valuable feature of PV loops is that various cardiac parameters can be calculated from just two measurements, LV pressure and volume. Some of these include: stroke volume (SV), stroke work (SW), cardiac output (CO), ejection fraction (EF), max and $\min \frac{d P}{d t}$, etc, outlined in Table 1.1] [40].

In addition, by plotting a series of loops, one can obtain valuable measurements of heart contractility and elastance. These are found by plotting the End Diastolic Pressure-Volume Relationship (EDPVR) line, End Systolic Pressure-Volume Relationship (ESPVR) line, and the Ea (Arterial Elastance) line. EDPVR describes the passive properties of the myocardium 


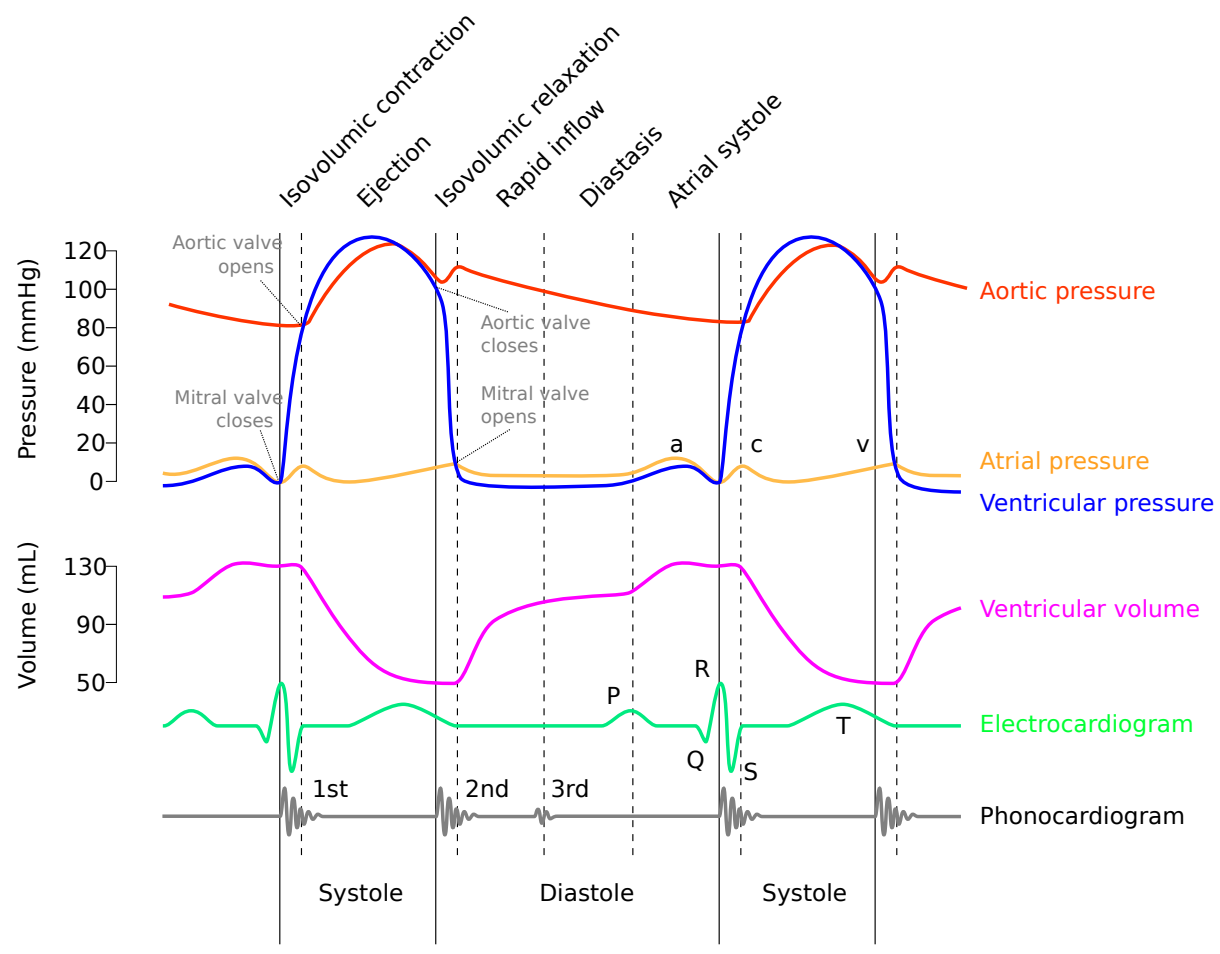

Figure 1.3: Traditional Wiggers diagram that shows the relationships between Aortic pressures, left ventricle pressures, left ventricle volume, and ECG. [1, 2] Licensed by CC BY-NC-SA 2.0

Table 1.1: Various cardiac parameters

\begin{tabular}{lll}
\hline Cardiac Parameter & Description & Formula \\
\hline SV - Stroke Volume & $\begin{array}{l}\text { Volume of blood being pumped } \\
\text { with each beat }\end{array}$ & $S V=E D V-E S V$ \\
\hline SW - Stroke Work & $\begin{array}{l}\text { Mechanical work done by the heart } \\
\text { muscle }\end{array}$ & $S W=$ Area inside PV loop \\
\hline CO - Cardiac Output & $\begin{array}{l}\text { Volume of blood being pumped by } \\
\text { the heart in 1 min }\end{array}$ & $C O=S$ V $x$ Heart Rate \\
\hline EF - Ejection Fraction & $\begin{array}{l}\text { Volumetric fraction of blood } \\
\text { pumped out of the LV with each heart beat }\end{array}$ & $\frac{S V}{\text { End Diastolic Volume }} \times 100 \%$ \\
\hline
\end{tabular}


and the slope of the EDPVR line represents the reciprocal of ventricular compliance [42]. If the ventricle is less compliant (stiffer), higher EDP will result at a given EDV. If compliance increases, EDV may become high but EDP may not increase substantially [42,43]. ESPVR describes the maximum pressure that can be developed by the left ventricle at any given volume, i.e, PV relationship at end of systole [42]. The slope of this line represents the end systolic elastance, which is used to provide a measure of contractility. Its volume intercept point is fixed as the left ventricle always has set amount of blood left after ejection. As the ESPVR shifts leftward, contractility increases, this results in increased SV, SW and ESP. If shifted rightward, contractility decreases, causing a decrease in SV, SW and ESP. The ESPVR is insensitive to changes in heart rate (HR), preload, and afterload, providing a highly accurate measure of contractility [44-47]. Preload is defined as the LV wall stress at end diastole and can be calculated directly from the PV loop. Another important paramater is Afterload which refers to the LV wall stress during ejection. The descriptions of these paramaters are just brief introductions into the topic of cardiovascular research and provide the reader with a basic understanding for this thesis. For additional reading, see [42,48]. The arterial elastance (Ea) line is a measure of the arterial load and can be pivoted (ie moved left or right at same volume intercept point) and/or can move volume intercept point [42]. By pivoting the Ea line up, there will be a decrease in SV but an increase in ESP, and if pivoted down, SV will increase but ESP will decrease. Additionally, by moving the volume intercept point left or right, there will be a decrease or increase in Preload, respectively. When Preload increases, ESP, SV and SW will increase and the opposite will occur when Preload is decreased. The analysis of in-vivo measured LV PV loops as an indicator of the cardiac functionality is remarkably useful, as it allows researchers to determine load independent indexes of contractility and chamber stiffness [37,49]. In addition, accurate real-time PV loops allow researchers to quantify cardiac pathology, e.g., congestive heart failure, by extracting stroke volume, cardiac output and various other parameters with just two physiological measurements, pressure and volume [37,38,50-55]. Currently, PV loop analysis is the gold standard for investigation of cardiac function with respect to clinical disease [56]. 
Cardiac contractility is an inherent property of the heart muscle that enables and affects the heart's ability to generate force [56,57]. Contractility can be altered by the nervous system, hormones, drugs or diseases and it is critical to understand the affects on the heart that changes in contractility can create. If contractility increases, the hearts energy consumption will increase causing increased oxygen consumption and increased blood flow [57]. If the patient has heart disease or other cardiovascular conditions, increases in blood flow and oxygen consumption can cause significant health issues. On the other hand, if contractility decreases, blood pressure will be decreased as well as blood flow. This can cause significant issues for patients with pre-existing heart conditions and can cause clinical heart failure. It is essential in drug studies to determine the affect on contractility that a drug has and to ensure that changes in contractility are discovered and accounted for, failure to do so could result in adverse heart effects or death. Currently, the FDA and the ICH (International Conference on Harmonisation of Technical Requirements for Registration of Pharmaceuticals for Human Use) provide guidelines to pharmaceutical companies for safety pharmacology studies, these guidelines are known as "The S7A: Safety Pharmacology Studies for Human Pharmaceuticals" [58, 59] and "The S7B: The Non-Clinical Evaluation of The Potential for Delayed Ventricular Repolarization (qt interval prolongation) by Human Pharmaceuticals" [60,61]. These two documents provide guidelines for which measurements should be done to assess the potential risks of novel pharmaceuticals for human use [57-61]. These guidelines only require the assessment of blood pressure (central arterial pressure), heart rate (HR), and electrocardiogram (ECG) in nonclinical in-vivo safety pharmacology studies. Contractility effects are not required by the guidelines and only are a suggestion for additional studies, thus may be missed if traditional measurements are used. Nevertheless, by measuring contractility during drug safety testing, will reduce the human risk during clinical trials and the possible failure of the drug to market.

Traditionally, two main methods are used to assess contractility in pre-clinical safety pharmacology using the suggested measurement techniques from the S7A guidlines: 1) ECG with blood pressure and 2) HR with blood pressure. The majority of researchers [62-64] use ECG 
with a LV blood pressure sensor to study the changes in the QA interval (measured from the Q wave point on the ECG to when the Aortic valve opens) and LV $\frac{d P}{d t_{\max }}$ (peak positive rate of pressure change inside the left ventricle) as an index of contractility change. Both, QA interval and LV $\frac{d P}{d t_{\max }}$ should change when contractility changes, however, some drugs can delay the ECG signal and not effect LV $\frac{d P}{d t_{\max }}$, thus, extend the QA interval and not actually change contractility [62-64]. This would provide a false assessment of contractility change and may effect other functions of the heart. In addition, $\mathrm{LV} \frac{d P}{d t_{\max }}$ is also affected by preload, afterload, and heart rate (HR) [65]. Consequently, changes in LV $\frac{d P}{d t_{\max }}$ may not directly indicate a drug's affect on contractility, rather its affects on preload, afterload, or HR. Furthermore, this method is known to capture reduced contractility more accurately than increased contractility due to the accuracy of the QA interval. Many drugs will directly or indirectly affect the HR, thus impacting $L V \frac{d P}{d t_{\max }}$. As a result, HR must be taken into account when using LV $\frac{d P}{d t_{\max }}$ to accurately determine changes in contractility. The second method, presented in [65], introduces a HR correction factor for $\mathrm{LV} \frac{d P}{d t_{\max }}$, producing a more accurate contractility assessment. This method does not account for loading conditions (preload and afterload), thereby possibly providing a incorrect assessment of changes in contractility.

As mentioned above, PV loops are used to measure many hemodynamic parameters and are considered the gold standard for assessing contractility in the heart due to ESPVR's insensitivity to preload, afterload, and HR [66]. PV loops are typically limited to clinical disease testing environments [67], however not until recently have they been used in safety pharmacology studies [67,68]. The main reason why the traditional methods for contractility assessment (LV $\frac{d P}{d t_{\max }}$, QA interval) are used over PV loops in safety pharmacology studies is the lack of a fully implantable PV loop measurement solution [65,67]. These traditional methods can be implemented in small implantable telemetry systems allowing many animals to be monitored remotely and in free moving environments. Currently, no fully implantable PV telemetry system has been implemented for Safety Pharmacology assessment applications. A fully implantable PV telemetry system would not only allow safety pharmacologists to better deter- 
mine a drugs effect on the heart over traditional assessment methods (heart rate, blood pressure, ECG) but would also improve drug efficacy with the additional physiological parameters that PV loops provide. Therefore, it is critical to obtain real-time PV telemetry data to allow for the calculation of these parameters instantaneously.

\subsection{Research Problem}

Left ventricle volume measurements in small laboratory animals is a difficult task to achieve due to the size restrictions and heart rate of these animals. For example, a small mouse heart's LV volume can be as small as $40 \mu \mathrm{l}$ and have a heart rate of 500-600 beats per min (bpm) with heart rates reaching as low as $300 \mathrm{bpm}$ for an anesthetized mouse [69]. In addition, the weight of these animals is quite small (mice: $20 \mathrm{~g}-40 \mathrm{~g}$, rats: $150 \mathrm{~g}-400 \mathrm{~g}$ ). This requires measurement systems to be quite small, impacting the effectiveness and overall functionality of the measurements. Methods to obtain PV data can be categorized into either non-invasive or invasive measurement techniques. Multiple non-invasive approaches on the measurement of instantaneous blood volume from small laboratory animals have been accomplished, these include echocardiogram [70,71], X-Ray computed tomography (CT), [72], Magnetic Resonance Imaging (MRI) [71,73-75], and piezoelectric crystals [76].

With each of these techniques, detailed images of the heart are created indicating the volume of the heart as well as many other physical features including possible tissue tears and the surrounding heart muscle. However providing very accurate LV volumes, these techniques require large-scale costly physical measurement equipment (CT scanner, MRI, etc.) along with imaging technicians to operate the equipment. Furthermore, extensive post-processing imaging methods are required to generate a detailed cardiac image. These factors prevent economical large-scale, long-term cardiac data collection. Currently, the predominate invasive measurement technique is the conductance catheter measurement method [70, 77, 78]. This technique provides a method for the accurate measurement of LV blood volume from small lab 
animals in a cost effective and accurate fashion.

To obtain cardiac PV data with the conductance method, a small catheter is inserted into the LV of the heart where measurements are taken. Pressure and volume measurements are captured separately by different electronic systems. A pressure measurement is acquired by a solid state pressure sensor located at the tip of the catheter where the pressure from the blood will react with the sensor to cause a potential difference representing the pressure signal [79]. A volume measurement is obtained using a four electrode catheter system inserted into the subject's LV to generate an electric field and to continuously measure the conductance or admittance from the animal which is converted to volume [80]. These PV catheters are connected to measurement systems to fully capture and process the cardiac data for physicians and researchers to use.

Commercial PV measurement systems employ a tethered approach to measure cardiac signals from small animals. A moderate sized PCB $(60 \mathrm{~mm} \times 35 \mathrm{~mm})$ is used as an interface from a conductance catheter to a larger external base station where main processing occurs, Fig. 1.4 [3]. Although accurate, this method does not allow for long-term and large scale realistic cardiac data collection from animal subjects as they are generally under anesthesia during the procedure, thus not allowing the animal subject to move naturally in their normal environment.

To solve this critical problem, implantable telemetry systems are used. Telemetry systems are able to capture, process and transmit specific information to an end device, wired or wirelessly. These systems contain sensors to measure the biomedical data from inside the living body and can be external, for example in ECG, EEG monitoring or implantable in the case of most cardiac monitoring, Fig. 1.5. Implantable telemetry systems are found in many different areas of medicine including diagnostics and monitoring [81], drug delivery systems [82], neurology [83], audiology [84], and cardiology [85].

Implantable wireless telemetry systems have many advantages over traditional wired solutions especially in safety pharmacology applications including: freely moving conscious ani- 


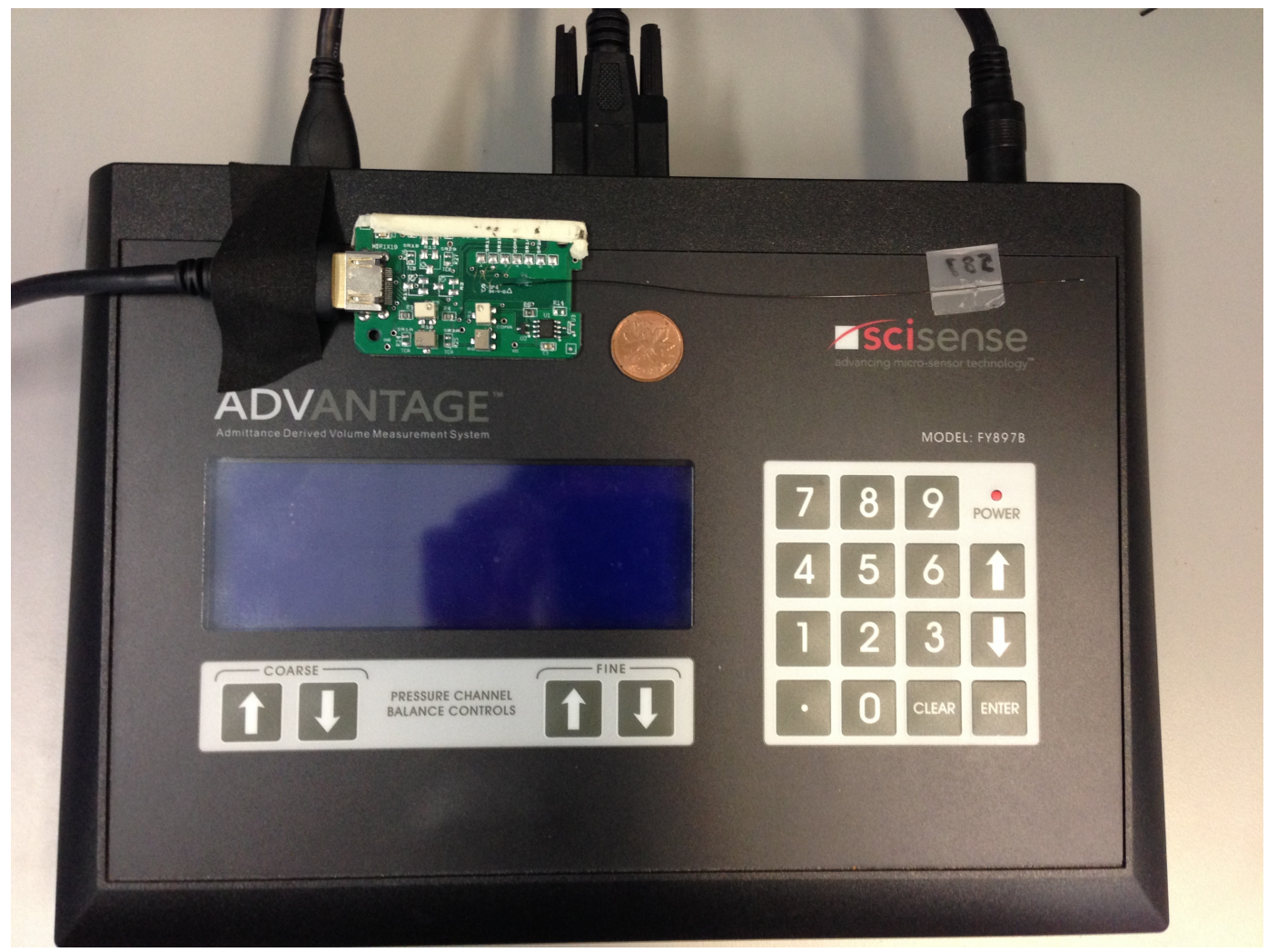

Figure 1.4: Commercial Pressure-Volume Tethered Measurement System from Transonic Scisense Inc. Interface Module, 1.2F Catheter, External Processing Unit [3]. 


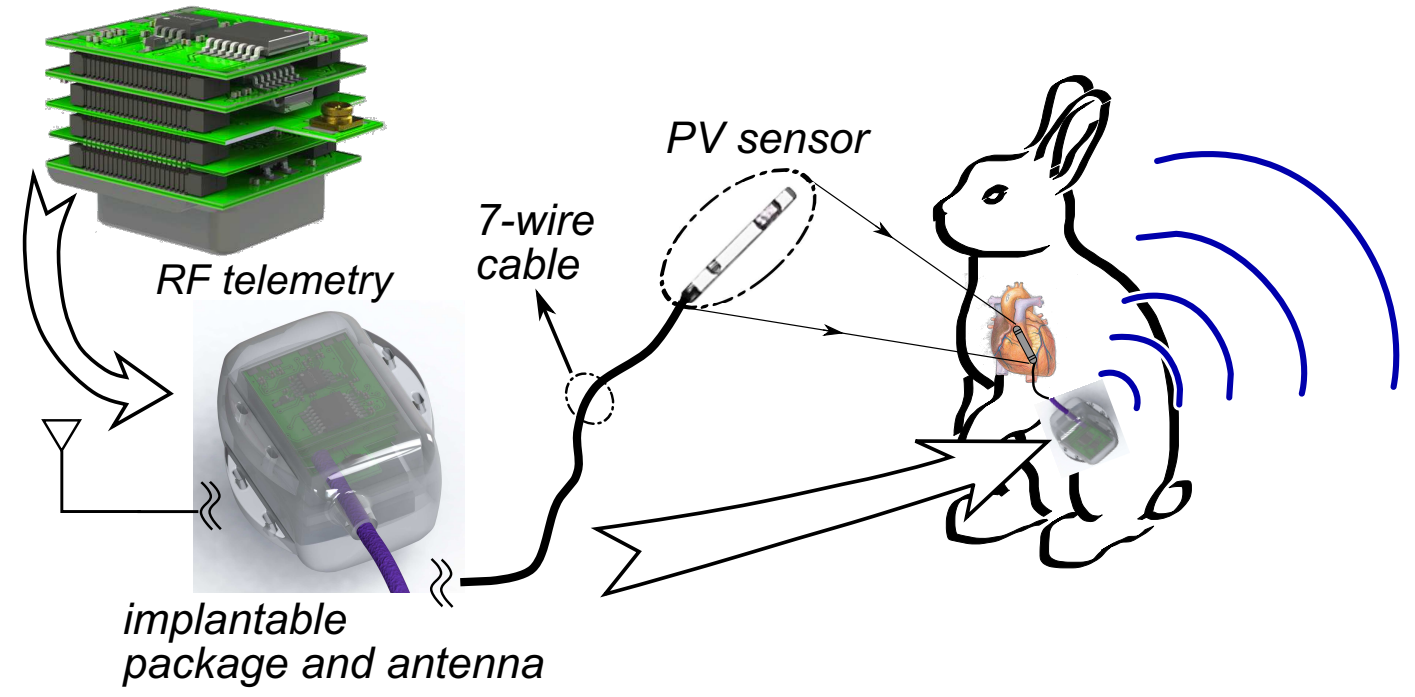

Figure 1.5: RF telemetry system shown inside a rabbit model. Note: implant location not to scale. [4,5].

mal data collection, chronic data collection, fewer measurement site infections, decreases costs as less personal are required, enhancement of safety of lab personal as fewer animals have to be physically handled to collect data, etc. Many issues exist with the current implementations of such systems including size restrictions, power consumption, reliability, number of sensors used etc. These issues are significantly more important for cardiac research due to the size of the animals involved.

A cardiac PV implantable telemetry system would contain all sensor electronics as well as power and data transmission electronics. However, realistic design constraints are driven by the subject's body size and the implant's power consumption. Larger subjects (e.g. bovine or human) can accept an implant whose volume is in the order of a few tens of cubic centimetres, while a tiny mouse body ( $20 \mathrm{~g}-40 \mathrm{~g}$ weight) can only tolerate a fully implanted object with the volume in the order of a few tens of cubic millimetres. Existing discrete components are not suitable for small subjects due to the package size. Thus, micro-sized implantable telemetry systems are an active area of research [79, 86, 87]. Power consumption is also of great importance to the implantable system design as decreased power consumption can lead to smaller battery sizes, further reducing the systems volume. A low-power telemetry system 


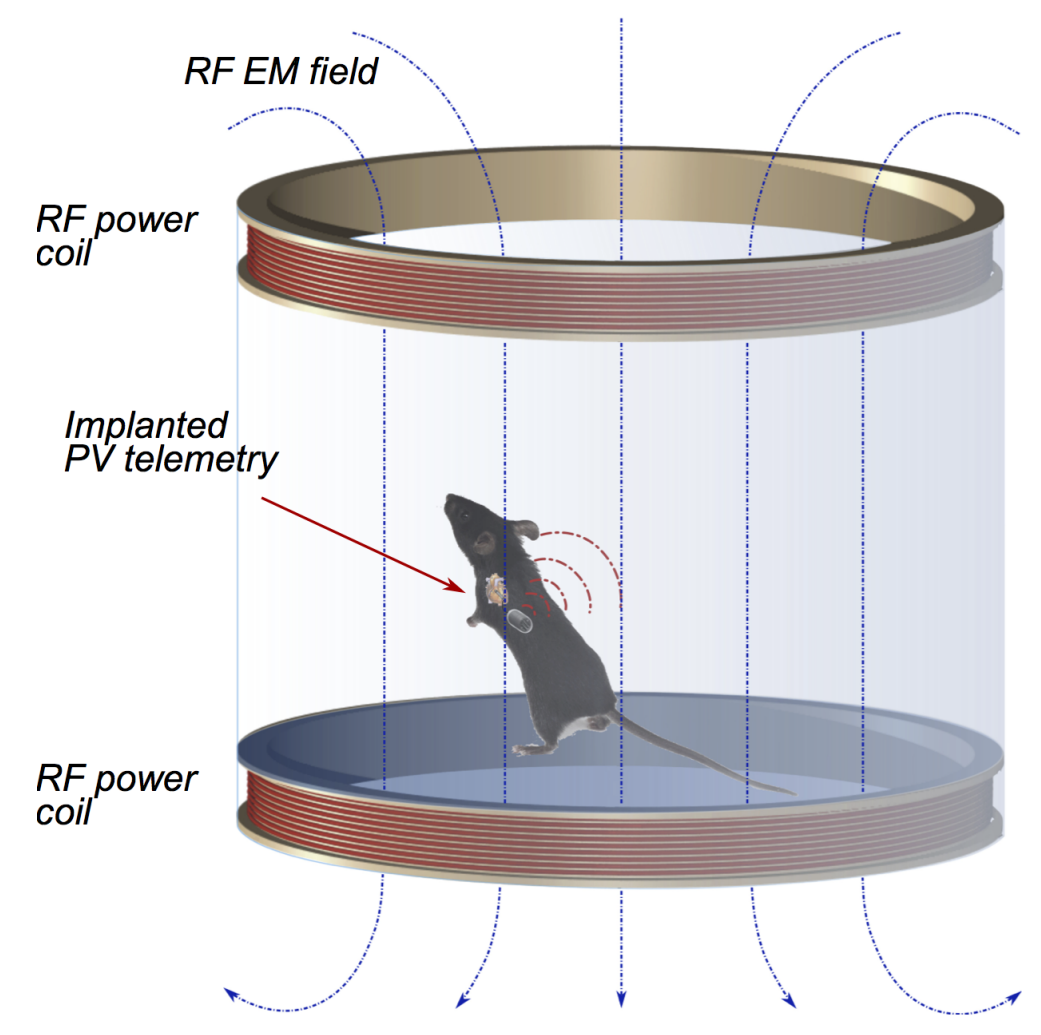

Figure 1.6: Wireless implantable system active cage measurement environment, [4]

is capable of being implanted for longer periods of time while operating off of smaller capacity batteries. Active measurement cages have been used to provide wireless power to the implants enabling long-term data collection without the need for batteries [21, 88], Fig. 1.6 .

Current state-of-the-art wireless cardiac telemetry systems typically employ blood pressure (aortic), ECG, and temperature sensors [21, 22, 89]. Although small and advanced, these systems currently do not measure left ventricular blood volume, thus cannot be used to measure PV loops. In order to generate accurate PV loops it is essential to measure both pressure and volume data at the same time, thus a dual sensor approach is required. Limited research has been completed on implantable PV telemetry systems in literature, where only four systems have been developed [23-25, 90], a detailed review of these systems along with other relevant works is provided in the following chapter. These devices are all limited by their size, weight, power consumption, and battery life to be implanted for long periods of time. It is extremely important to develop a fully implantable cardiac pressure-volume telemetry system 
that is small enough to fit into mice and rats. This would enable researchers to continuously monitor cardiac health by collecting real-time chronic PV data from freely moving conscious animals. Thus, enabling safety pharmacologists to utilize the gold standard of PV loops in their drug studies. By using PV implants along with other diagnostic tools, pharmaceutical companies can create safer and more effective drugs for human use.

\subsection{Research Objectives}

The aim of this thesis is to develop a miniature, low-power wireless implantable telemetry system that is designed to measure left ventricular pressure and volume physiological data. This device should be suitable for small to medium sized animals (e.g., rats, rabbits). The objectives of this thesis are:

- Existing commercial PV telemetry systems rely on tethered data from large external processing units, preventing natural environment data collection, hence an implantable telemetry system is required. This system should contain all lower level blocks to allow for data collection, processing, and transmission to an end device for post processing. To acquire pressure-volume measurements, a sensor interface must be developed to deliver excitation signals to the test subject and convert the measure voltages to valid application data.

- The implantable system must be very small and light weight to ensure no impact on the animal subject. Therefore, external components should be minimized and stateof-the-art printed circuit board (PCB) manufacturing techniques should be employed. Furthermore, different packaging technologies should be explored to create an optimum implant enclosure for the telemetry system.

- A realistic phantom tissue is required to evaluate and characterize the system prior to implantation. This phantom should have similar electrical properties to animal tissue, 
allowing RF transmission to be evaluated. In addition, the system must be calibrated to ensure valid measurement data.

- Design a measurement application to receive physiological signals and log data. An external base station is required along with a software interface to view, collect, and analyze the data.

- Evaluate the implantable PV telemetry system in-vivo in a non-anaesthetized test subject. Live physiological signals should recorded and compared with existing recording techniques.

- This research should advance the knowledge of implantable medical devices by providing a detailed literature review and insights on the trends in this research field.

\subsection{Organization of the Thesis}

In this thesis an implantable telemetry system is developed and manufactured that measures both left ventricle pressure-volume. The telemetry system is tested and evaluated in-vitro and in-vivo in a free moving swine.

In Chapter 2, background info on implantable medical devices is presented, discussing application areas, challenges, and other important concerns in the design of implantable medical devices. Furthermore, background on blood pressure and blood volume measurement techniques in small laboratory animals is presented. State-of-the art in pressure-volume systems is discussed in this chapter.

In Chapter 3, a comparison study of all three conductance-to-volume equations along with the accuracy of the analytical model of the conductance catheter is presented. In addition, the overall accuracy of the admittance catheter volume measurements is studied.

In Chapter 4, the wireless telemetry system design is discussed in detail. The system captures, processes PV data and transmits this data wirelessly to an end base station for further 
processing.

In Chapter 5, the in-vitro (non-implanted, external of body) characterization of the implantable telemetry system is presented. A phantom tissue study to model the effects of implant conditions, followed by details of the enclosure design and study. Calibration of the system is also presented.

In Chapter 6, details of the in-vivo study are presented. The study was designed to evaluate the PV system in a non-anaesthetized test subject, i.e., freely moving animal. In addition, the study was completed to determine initial catheter-heart interaction. Furthermore, a comparison of previous works is discussed in this chapter.

The research work is summarized in Chapter 7. Main achievements and milestones are listed, and suggestions for future work are presented. 


\section{Chapter 2}

\section{Implantable Medical Devices}

In the previous chapter a brief overview of the main research problems, motivation, and objectives for this thesis were stated. In this chapter, implantable medical devices are introduced providing the reader with a general knowledge of the typical applications, challenges, and research problems that exist in this field. Pressure and volume measurement techniques in small laboratory animals are discussed in Section 2.2 and 2.3. In addition, a discussion of state-ofthe-art in both implantable pressure telemetry systems as well as those that incorporate both pressure and volume sensors. This background knowledge provides a basic understanding of the application subject and the required theoretical knowledge for the remaining chapters in this thesis.

\subsection{Overview of Implantable Medical Devices}

An implantable system is generally referred to as any device/system that is intended to function inside a living body for either short or long term use [91]. Implantable systems provide a method to improve the quality of life of living beings as well as provide monitoring solutions for various medical applications. The specific advantages of implantable devices in comparison to other monitoring methods such as magnetic resonance imaging (MRI), and CT scans are their small size [92], low weight [93], low power [94], and increased functionality [92]. As 
these micro-systems are dealing with living bodies, they require additional research into areas such as packaging [95], reliability [96], and bio-compatibility [97]. To achieve these various measurements, different types sensors are utilized to convert the bio-signals to electrical data for processing. One of the most common sensors used is a pressure based sensor. These different sensors are utilized in many application areas such as retinal [87,98], neurological [83, 86, 99], and cardiac [85]. In addition, the focus on power consumption, small size and wireless power are common themes found throughout the different application areas that implantable telemetry systems are utilized.

For example, one of the more recent advancements in retinal implantable systems has been achieved by Shih [94]. Here, Shih presents a $2.3 \mu \mathrm{W}$ wireless intraocular pressure/temperature implant. This implant is used to monitor intraocular pressures that are used in the diagnosis and treatment of glaucoma. In addition, Yang [98] has studied inductor modelling to wirelessly power such retinal implants. These retinal implantable systems have the potential to greatly improve the monitoring of such diseases as well as the possibility of early detection before current diagnostic methods can.

Another major application area for implantable systems is in neurological research. For instance, Neihart [86] presents a low-power bi-directional telemetry system that is designed to be fully implanted inside a human body for neural recording applications. Additional research was achieved by Ghovanloo [99] who developed an implantable system which contained a multichannel mircostimulating system-on-a-chip (SOC). This system was designed to work as a neural protheses. These systems are able to emulate natural neurological functions that are damaged and provide rehabilitation to the patient. Auditory and visual prostheses are examples that this device strives to emulate. Furthermore, Lee [83] presented an inductively powered wireless neural recording system, that contained a $14.19 \mathrm{~mm}^{2}$ integrated circuit (IC) that consumes $5.85 \mathrm{~mW}$. This publication focused on the wireless power transfer of the custom IC and system.

More recent research has been completed by Greenwald et al. [6] who developed a low- 


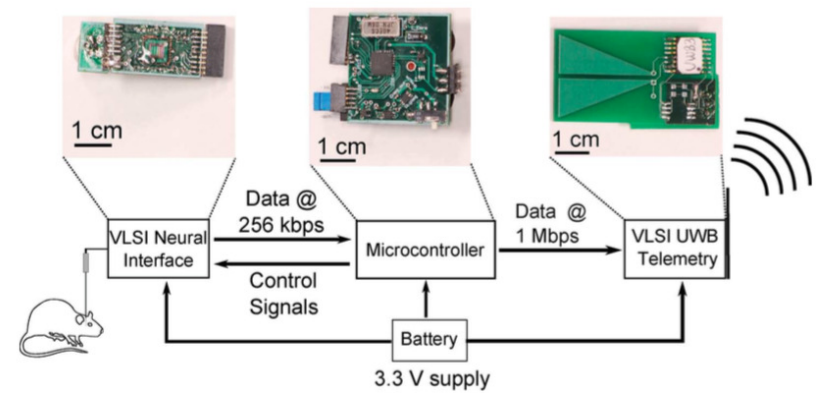

(a)

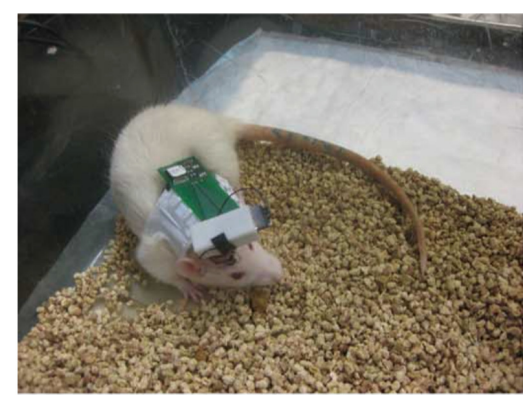

(b)

Figure 2.1: Implantable Neural Monitoring System by [6] where a) the block diagram and PCB implementation; b) system on mounted on rat's head. Images from [6]. Copyright (C)2011 IEEE.

power neural monitoring system for small animals that contains three PCBs configured in a headstage with a weight of $24 \mathrm{~g}$, Fig. 2.1. This device was evaluated in-vivo in awake rats. Zhang et al. [100] improved upon this research by creating a smaller, lighter backpack device for neuro recording at $10 \mathrm{~g}$ and sub $1 \mathrm{~cm}^{3}$, however, battery life was greatly reduced to achieve the smaller sized device. The most recent advancements in neuro recording have come from Gagnon-Turcotte et al. [7] and Liu et al. [8]. In [7], they present a wireless headstage for combined optogenetics and multichannel electrophysiological recording. The headstage is able to perform readings and stimulation simultaneously. The headstage itself is $3.06 \mathrm{~cm}^{3}$ in volume and weighs $2.8 \mathrm{~g}$, both volume and weight are measured without battery. It is designed with a foldable semi-ridged structure and is placed on the mouse's head while in use, Fig. 2.2. In [8] an optimized wireless sensing neural recording system is presented. Their system includes a custom SOC with integrated ADC, filters, and sensing module. The implant is placed under the skin on the head where wireless power and data is transfered from an external device placed on the exterior of the skin, Fig. 2.3. Although only tested in-vitro, this system has the capability to become a fully integrated neural measurement solution for monkeys. Another area of research in neurological implants is microstimulation. Two of the more recent publicatons in this area are from Azin et al. [9] and Khalifa et al. [101]. In [9] a miniaturized system for spike-triggered intracortical microstimulation in rats is presented. Azin's system operates on 


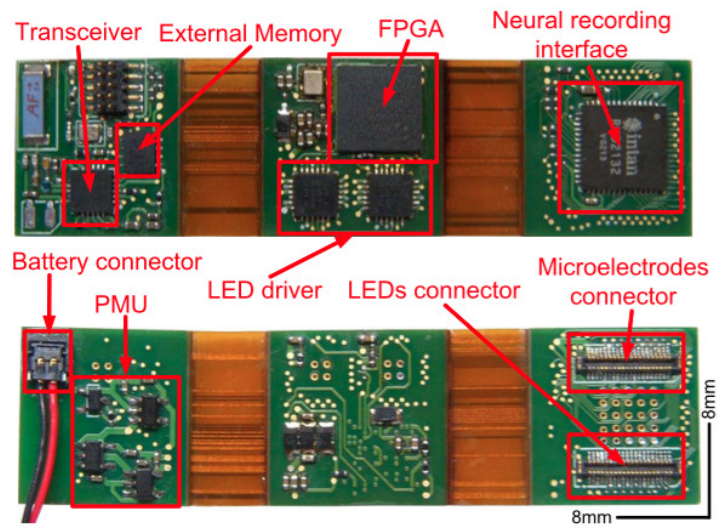

(a)

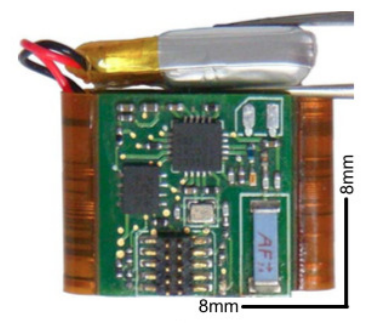

(b)

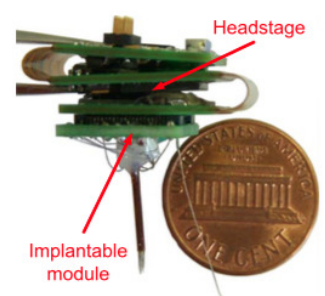

(c)

Figure 2.2: Wireless headstage for combined optogenetics and multichannel electrophysiological recording by [7] where a) is the two-sided view of the rigid-flex system, b) top system view folded with battery, c) side view attached to the implantable electrode module. Images from [7]. Copyright (C)2016 IEEE.

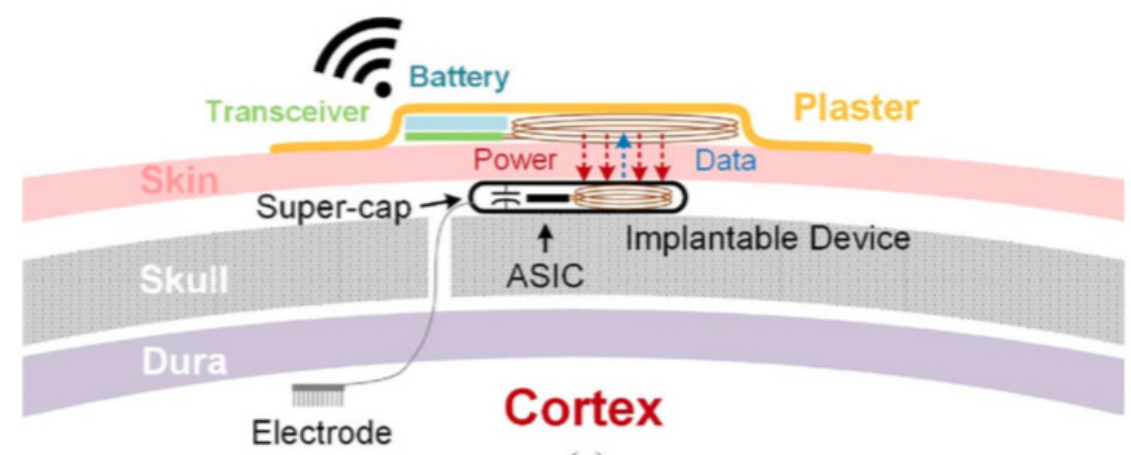

(a)

Figure 2.3: Implantable neural recording system proposed by [8] where the SOC is located under the skin with a larger external device located on the head of the subject. Images from [8]. Copyright (C)2016 IEEE. 


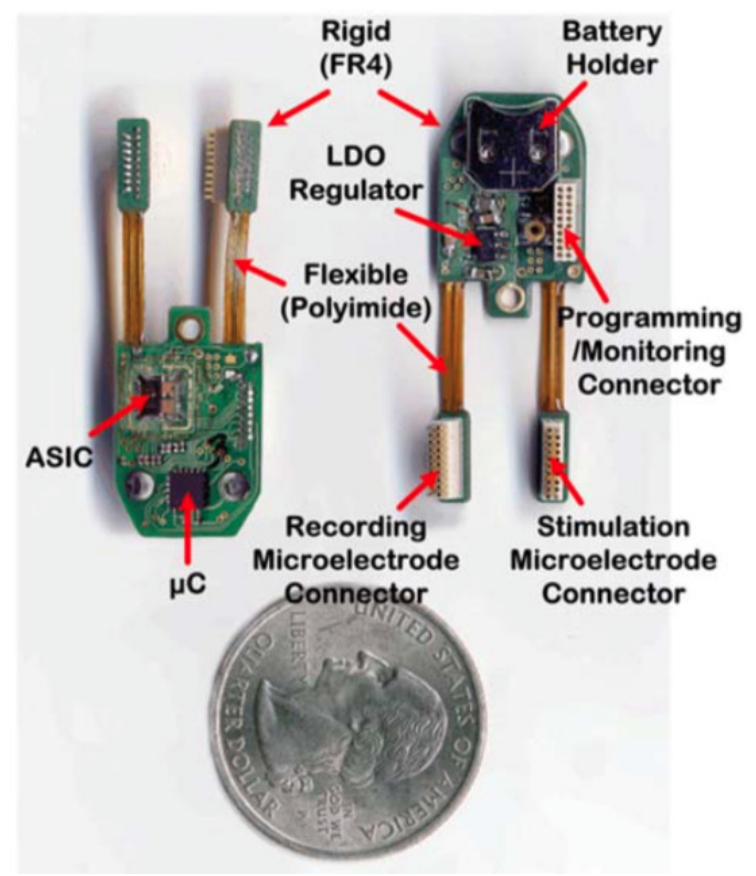

Figure 2.4: Implantable system by [9] for spike-triggered microstimulation in rats. Images from [9]. Copyright (C)2011 IEEE.

battery power with a volume and weight of $2.8 \mathrm{~cm}^{3}$ and $1.7 \mathrm{~g}$, respectively, Fig. 2.4. Most recently, Khalifa presented initial results in a novel microstimulator IC that consumes $16 \mu \mathrm{W}$, however, this system has yet to be tested in-vivo. Neurological implantable systems represent one of the most active areas of research [102-105], however, significant challenges still exist including miniaturization and power consumption. Implantable systems for neurological research provide methods to monitor and stimulate brain functions to improved diagnosis and long term health care of patients with neurological disorders.

Implantable systems are actively researched in other application areas as well such as blood glucose monitoring [106], drug delivery systems [107], and blood flow measurements [108]. A wireless implantable microsystem for blood glucose monitoring is presented in 2009 by Ahmadi [106]. Furthermore, Georgiou's group from Imperial College London is working on an artificial pancreas for treatment of diabetes, Fig. 2.5, [10, 109]. This is a closed-loop system that incorporates a glucose sensor, controller and insulin pump. These types of implants allow diabetic patients to monitor their glucose levels in real time and can help reduce symptoms 


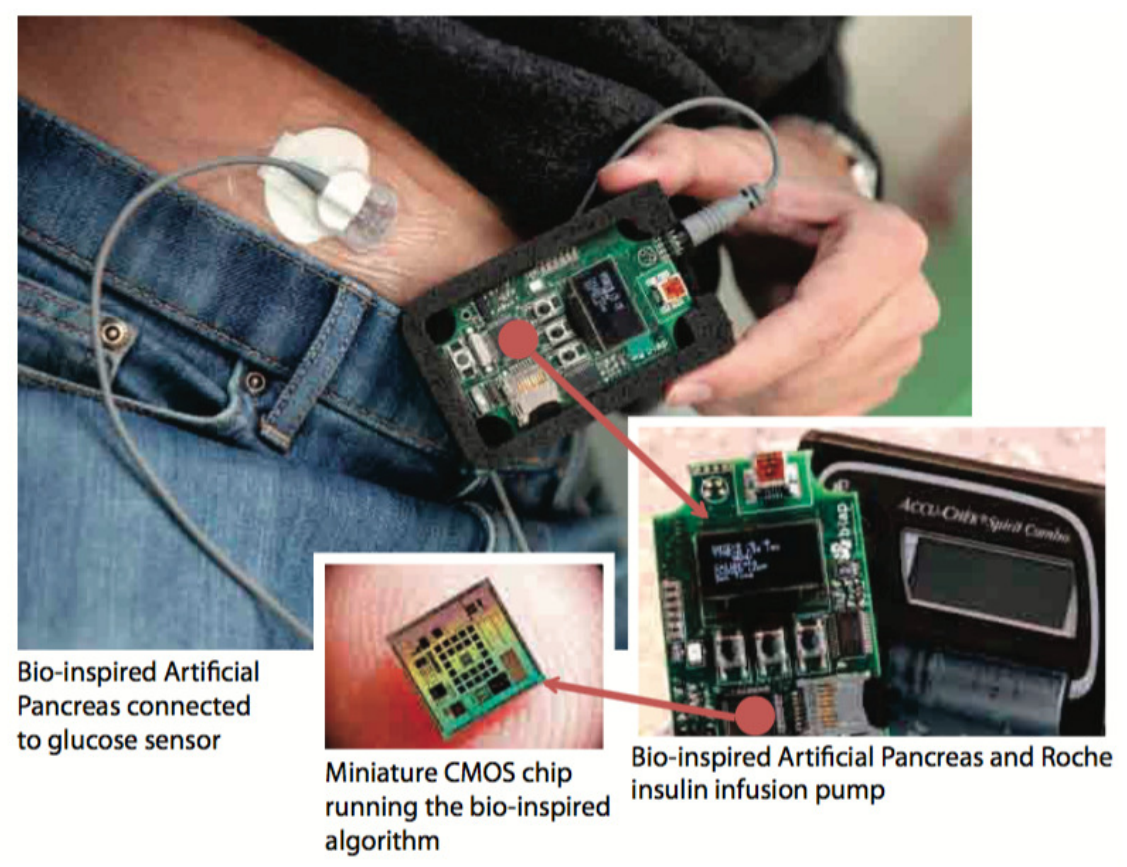

Figure 2.5: Artificial pancreas for treatment of diabetes from [10]. Images from [10]. Copyright (C)2014 IEEE.

associated with diabetes [110]. Biosensors are also a heavily researched area for implantable medical devices [11,111, 115]. One such example was developed by Baj-Rossi [11] et al. Here she presented a fully implantable system for remote monitoring of glucose and acetaminophen that was tested in-vivo in mice, Fig. 2.6. The implant itself contained custom ICs for measuring and processing the molecule concentrations as well as wireless data and power. In addition, a detailed examination into implant bio-compatibility was also provided in this paper. A full litrature review on biosensor development is found in [115]. Related to the development of biosensors are drug delivery systems. Smith [107] proposed a miniaturized drug delivery system for delivering medications to the eye. These types of systems allow doctors to control the exact dosage and delivery areas for maximum effectiveness.

In 2010, Khannur [108] presented an implantable IC for blood flow measurements within prosthetic grafts. Additionally, Lee et al. [12] developed a programmable implantable telemetry system for cardiac pacemaker applications. This device features a fully wireless recharging system and a custom IC to perform measurement and stimulus actions, Fig. 2.7. The device 


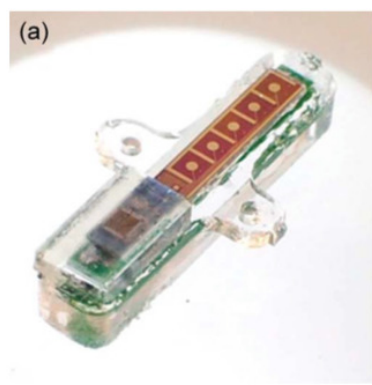

(a)

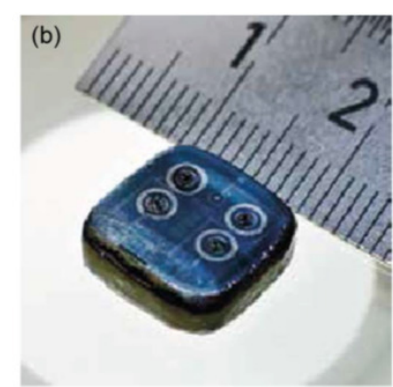

(b)

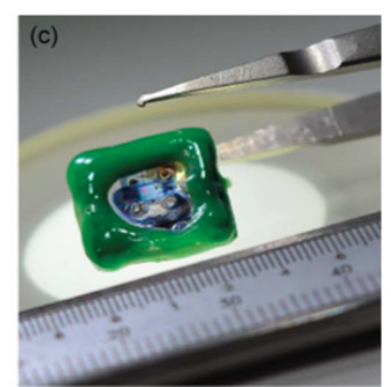

(c)

Figure 2.6: Three versions of Baj-Rossi's fully implantable remote monitoring system [11]. Implant contains custom IC measuring and processing the molecule concentrations as well as wireless data and power circuits. Images from [11]. Copyright (c)2016 IEEE.

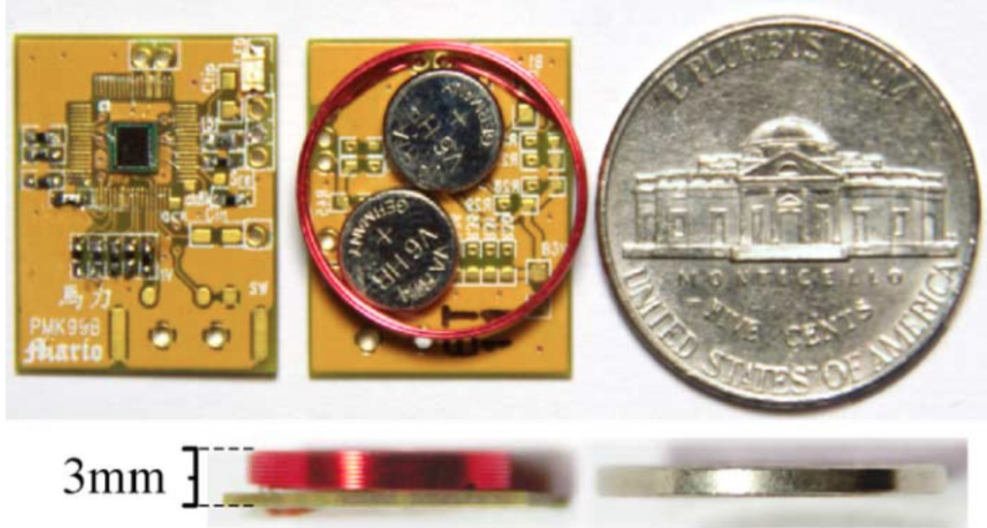

Figure 2.7: Implantable telemetry system for cardiac pacemaker applications designed in [12]. Images from [12]. Copyright (C)2011 IEEE. 


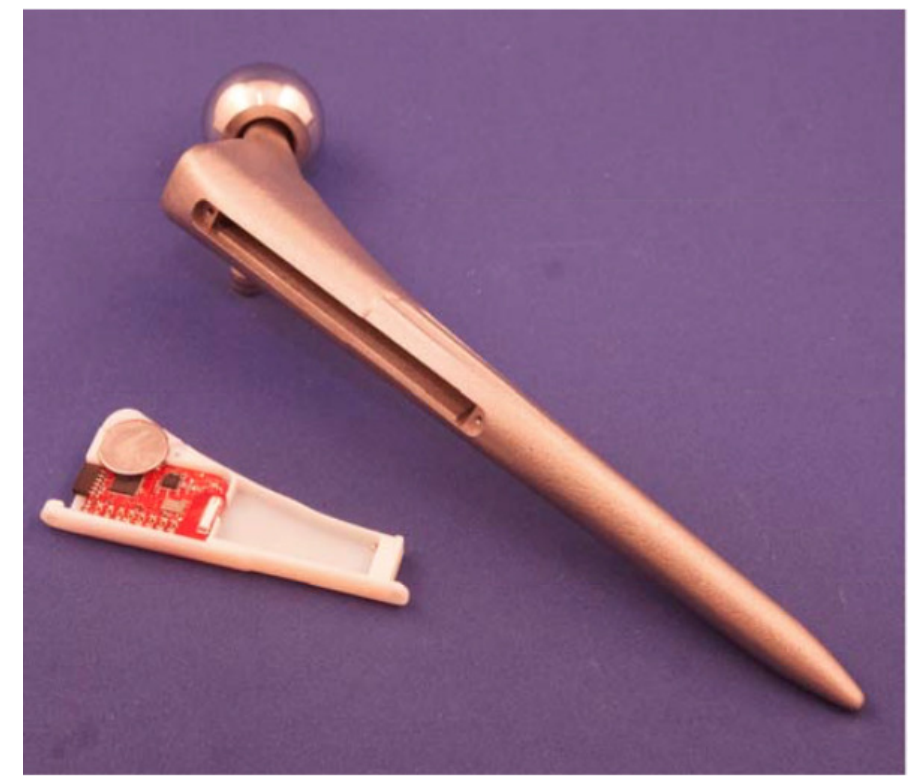

Figure 2.8: Smart hip implant proposed by [13]. Images from [13]. Copyright (C)2016 IEEE.

was tested in-vivo in large rats, with reported power consumption of $48 \mu \mathrm{W}$. These systems allow doctors to monitor and create better treatments for vascular based diseases. A full review of blood pressure and blood volume systems is provided in Section 2.2 and 2.3 . Implantable systems have also been developed for other application areas including smart pills for the gastrointestinal tract [116] and artificial bladder applications [117, 118]. More recently, implantable systems are beginning to be developed for hip and bone applications [13], Fig. 2.8 In addition to the major application areas above, much research is being conducted on specific features of implantable telemetry systems such as antenna design [102, 119, 120], analog front end circuits [121, 122], wireless powering [113, 123, 124], and RF transmitters [81, 125].

\subsubsection{Implant Design Challenges and Concerns}

Implantable medical device designers are presented with numerous design challenges and concerns when developing an implantable solution for their specific medical application. This section will introduce a few of the main challenges including power management, data transfer, security, and packaging.

Power management and implant powering are of great concern to medical device manufac- 
tures as implants are required to remain functional for long periods of time while collecting data. Traditionally, medical devices are powered off of small batteries which offer limited power, high toxicity, and must replaced in chronic long term patients, i.e., cardiac pacemakers. These batteries are typically replaced via a surgical procedure that can cause complications with patients. Research into different battery technologies has yielded smaller, higher density batteries, however, these are still only available in research applications [126-129]. Higher power applications including pacemakers, neurostimulators, etc, will often utilize rechargeable batteries to extend the life of the implant. These can be recharged using wired or wireless methods.

An alternative to battery power is to use wireless energy transfer and energy harvesting techniques. Wireless energy transfer typically refers to an inductive link involving small coils, power amplifiers, an external power source, and AC to DC circuits [129, 130]. The link must be optimized for power efficiency to ensure maximum power transfer. This process is not trivial as all factors of the design must be accounted for including the tissue medium [113,131133]. Additional power transfer technologies exist including Ultrasound [134,135], Resonance based coupling where coils are resonant at a specific frequency allowing transfer range to increase versus traditional inductive coupling [131,136], RF [133 137], etc, each with their own advantages and drawbacks. A more recent field of research is in direct energy harvesting from within the body. Energy harvesting refers to harvesting ambient energy from another source that is not actively delivering power to the implant [138-141]. Examples of power harvesting solutions published in literature are optical harvesting [138], bio-fuel (glucose) [139], thermal [140], and kinetic [141]. The main drawback of power harvesting solutions is the limited power density achieved, typically in the sub $20 \mu \mathrm{W} / \mathrm{cm}^{2}$ range. This power density is not practical for todays high power implants, thus these solutions are typically part of a rechargeable battery solution or powering the sensor interface only [142]. The implant's size, function, expected lifetime, distance from base station, and reliability all determine the power source (battery, power transfer, energy harvesting) used [143]. This is significantly important for small animal 
implant design as the size is severely limited, thus the applicable power sources are limited [4]. Nevertheless, small animal power transfer is an active area of research focusing on coil misalignment [136] and full power cage solutions [21, 126, 127, 144-146].

Another design consideration is wireless data transfer from the implant to the external base station. In order to determine the operation frequency for data transfer, implant designers must first evaluate the final implant location to determine the tissue properties for which the wireless signal must pass through. Lower frequencies pass through tissue better than higher frequencies, however, the structure of the medium (muscle, fat, bone) will also dictate the frequency choice [147--149]. The application will determine the data-rate required for data transfer which will impact frequency choice. Finally, the size of the implant must be considered as higher frequencies $(2.4 \mathrm{GHz})$ allow for much smaller antennas than lower frequencies (915 $\mathrm{MHz}, 13.56 \mathrm{MHz}$ ). In addition, frequency choice will impact power consumption. Although, one may be able to find an optimal frequency of use, only specific frequencies mandated by the FCC are for use in medical applications [150, 151], known as the Industrial, Scientific, and Medical (ISM) band [152]. The most common ISM frequencies used in implantable medical devices are 13.56 MHz, 433 MHz, 915 MHz, and 2.5 GHz [150]. Another frequency band designed specifically for small low-power, low-bandwidth implants is the MedRadio band at various points in the $400 \mathrm{MHz}$ frequency range [151]. Most of the research in this band is on custom MedRadio antennas for implant applications [153,154]. Therefore, a trade off must be made between the required data rate, antenna size, tissue absorption, allowed frequency and power consumption when deciding operational frequency.

Although much consideration goes into the operational frequency and power supply choices, data security is often overlooked. Implantable medical devices commonly use existing wireless protocols for data transfer, bluetooth, MSK, FSK, OOK, etc, which are unencrypted and can be viewed with standard electrical equipment [155]. This may not be a large concern for animal testing, not withstanding safety pharmacology studies, however, this poses a large security and privacy issue for human implantable devices. Furthermore, it is not only the data collection se- 
curity issue, but the base station to implant data can also be intercepted and compromised. It is common for pacemakers, drug delivery devices, insulin pumps, and other implantable devices to receive external commands, thus the possibility of an external attack, for example cause a electric shock from a pacemaker [155]. Currently, the limited power and range of implantable devices removes much of the the data threat, however, newer longer range devices are prone to attack. Encryption is not commonly used on implantable devices due its complexity, power consumption, and slower data rate [155], however, recently memristors have shown promise to be used in implantable encryption schemes [156,157]. Implant designers must be aware of all data security and privacy concerns when designing medical devices.

Implant designers are also faced with the challenge of medical device package design. The package of an implantable system must protect the internal electronics from being damaged and must also protect the living body from tissue damage and other undesirable effects caused from mechanical, thermal or biological stresses, [11,158]. The harsh environment puts extreme requirements on the overall system design, including electronic and package design. For example, the design of an intraocular implant is bound by the specific shape of the eye impacting the electronics and antenna design [85]. However, the volume of the eye cavity is still suitable for implanting a high frequency antenna [94]. Furthermore, research into custom antenna solutions, e.g., flexible electronics, remains a heavy area of research [159,160]. Custom solutions allow for the system design to be minimized for the specific implant application, that is, to reduce the size and weight of the overall implant. Thus, biomedical implant package design is a very important stage in the design process. Every implant designer is faced with these issues and must determine which is the most critical to their particular application.

\subsection{Blood Pressure}

Blood Pressure is one of the most important vital signals [161] in the study of the living body. Blood pressure refers to the force exerted by circulating blood on the walls of blood vessels 
[162]. Pressure can be measured in various parts of the body including the aorta, known as arterial blood pressure [42], the left ventricle [163], the femoral artery [42, 164], intracranial [165], etc. Recall from Chapter 1 that the relationship between aortic pressure, LV pressure, LV blood volume, and ECG is described using the traditional Wiggers diagram, Fig. 1.3 .

Typically, when referring to blood pressure, without the measurement location known, arterial pressure is assumed. This is generally the case when referring to human blood pressure measurements, where a pressure cuff located on the upper arm and is used to measure systolic and diastolic (max and $\min$ ) pressures. This measurement represents the pressure exerted within the brachial artery [42], and is slightly different if measured directly in the Aorta. More specifically, the brachial pressure typically has higher systolic and lower diastolic pressures compared to a direct aortic pressure measurement [42]. A brachial artery or aortic pressure measurement provides doctors with valuable health information and can determine if the patient has hypotension (low blood pressure) or hypertension (high blood pressure) further determining their risk for cardiovascular desease. Similar to brachial pressure, the pressure in the femoral can be measured with a pressure cuff and can indicate the onset of various diseases including peripheral vascular disease [166, 167]. Femoral pressures exhibit slightly higher magnitudes of pressures compared to brachial pressures.

More importantly, the measurement of left ventricle (LV) pressure can provide insight into cardiac contractility. As mentioned in Chapter 1, cardiac contractility is very important when determining the safety of a new drug in the field of pharmacology safety. Furthermore, measuring LV pressures provides researchers with the most accurate real-time measurements of the heart filling cycle. Various important cardiovascular parameters are extracted from LV pressure measurements including End Diastolic Pressure (EDP), End Systolic Pressure (ESP), etc. One important LV pressure parameter is $\frac{d P}{d t_{\max }}$, known as the peak positive rate of pressure change inside the left ventricle $[34,168]$. As mentioned in Chapter $1 \frac{d P}{d t_{\max }}$ is one of the traditional methods to measure contractility. To measure $\frac{d P}{d t_{\max }}$ accurately, the sampling rate of the pressure measurement circuit must be much higher than the signal of interest. The remaining 
subsections will focus on measurement techniques for left ventricle pressure in animals

\subsubsection{Blood Pressure Measurement Techniques in Animals}

Blood pressure monitoring has been around since 1733 [48, 169] and has advanced from invasive wired techniques [170] [171] to fully wireless solutions [79]. In 2005, the American Heart Association (AHA) published recommendations for measuring blood pressure in animals [168, 172]. The AHA compared both indirect and direct measurement methods. Direct LV pressure measurement methods include fluid-filled catheters [173], MEMS based catheters [174], and full implantable systems [79]. Indirect pressure measurement methods for animals typically use a tail cuff device [175-177]. Direct methods allow for continuous real-time measurements whereas indirect methods typically will only provide major changes throughout the measurement cycle.

\section{Indirect Measurement Methods}

Traditional indirect measurement techniques for blood pressure monitoring include Doppler sphygmomanometry [178,179] and oscillometry [180-184]. In both approaches, a small cuff is placed around the animal's tail to measure the arterial blood pressure, similar to a human blood pressure cuff. Unlike when humans use blood pressure cuffs, animals require confinement and restraint that can create stress on the animal, further impacting measurement results [34, 184]. Furthermore, the blood pressure cuff must be located along the same plane (height) as the bottom of the heart to achieve accurate pressure readings which is difficult to achieve in laboratory animals compared to a human [34]. As opposed to pressure sensors inside of catheters, tail cuffs cannot obtain continuous blood pressure measurements, only systolic blood pressure data [79] and mean arterial pressures [183], thus non-LV pressures. Moreover, due to the infrequent measurement cycles of pressure cuffs, minor changes in blood pressure will not be detected. These methods are typically much less expensive and non-invasive than the direct based methods. A full comparison between Doppler and Oscillometry indirect pressure 
measurement methods is found in [179, 185, 186].

For larger animals, a newer measurement method known as high definition oscillometry (HDO) can be used [34, 187]. This technique still relies on a tail or leg cuff to perform the measurement, however, it is able to more accurately detect systolic, diastolic and mean arterial pressures over Doppler sphygmomanometry and oscillometry [34,188]. In addition, this method provides a quicker measurement rate and allows for measurement data to be viewed on a computer screen, allowing measurements to be rejected if required [34, 168, 188]. Various studies [176, 177, 187, 189] have been completed comparing HDO to direct methods for measuring arterial pressures. These studies indicate good correlations in arterial pressures when movement is kept to a minimum. As with traditional tail cuff methods, if the animal moves while measurements are taken, artifacts will be created on the measurements [34,190]. Additional stress on the animal can occur if extra restraints are involved, further impacting blood pressure results [34,190].

All three of these indirect methods will only provide systolic, diastolic and mean arterial pressures and will not provide LV pressures. Thus, indirect methods cannot be used when creating a LV pressure-volume loop, only direct methods can provide real-time LV pressures.

\section{Direct Measurement Methods}

Direct methods refer to measuring blood pressure at the specific internal location, i.e., arterial pressure [177, 191], femoral pressure [192], LV pressure [41], etc. In addition, the blood itself is in direct contact with the measurement device. The direct pressure measurement methods can be separated into two distinct categories: 1) catheters with external signal processing and conditioning; and 2) fully implantable telemetry solutions. Direct measurement methods allow for real-time monitoring of blood pressure such that minute changes in pressure can be detected [172]. This method allows researchers to obtain more comprehensive measurements for their studies. 
Catheters with external signal processing and conditioning: One of the first recorded direct pressure measurements was performed by Stephan Hales in 1733 [48, 169] where he measured arterial pressure in a horse. This was an extremely crude measurement where a glass tube was directly inserted into the femoral artery and the height of the blood in the tube above the LV of the blood was recorded. He measured both systolic and diastolic arterial pressures with this procedure [34]. The next major improvement in direct pressure monitoring was in 1828 when Poiseuille invented the U-Tube mercury manometer [48] where pressure measurement units $(\mathrm{mmHg})$ were first used. The U-Tube was replaced by a strain gauge pressure transducer that was connected to a fluid filled catheter by Statham in 1943 [15,34]. The system used by Statham and solutions similar to this had various limitations including low frequency response, pressure delay through the catheter tip to the transducer, etc [34].

To solve these issues, researchers moved the pressure sensor directly to the tip of the catheter to make pressure measurements. This was first completed by Van Citters and Franklin in large animals in 1965 [193]. Here, the sensor was placed at the tip of the catheter with additional external wires to complete the measurement. These fluid filled catheter tip sensors have improved and miniaturized greatly over the years and have been used in numerous animal studies [173, 191, 193, 195].

With the arrival of MEMS (Microelectromechanical Systems) based pressure sensors, traditional fluid-filled catheter pressure solutions have been replaced. These systems utilize either solid-state resistive [196] or capacitive [197] sensors to measure blood pressure. The sensors are assembled on the tip of the catheter and create an electrical voltage when the blood pressure changes. As with the fluid-filled catheters, these require a surgical procedure to guide the sensor to the measurement location, an invasive procedure. The first solid state based pressure sensor solution was invented in 1989 by Brockway et al. [14], Fig. 2.9. It was connected to a short fluid-filled catheter to complete the measurement [14, 15].

Solid state pressure sensors have rapidly advanced to their current form of using MEMS based sensors and are now the gold standard when measuring blood pressure in animals. These 


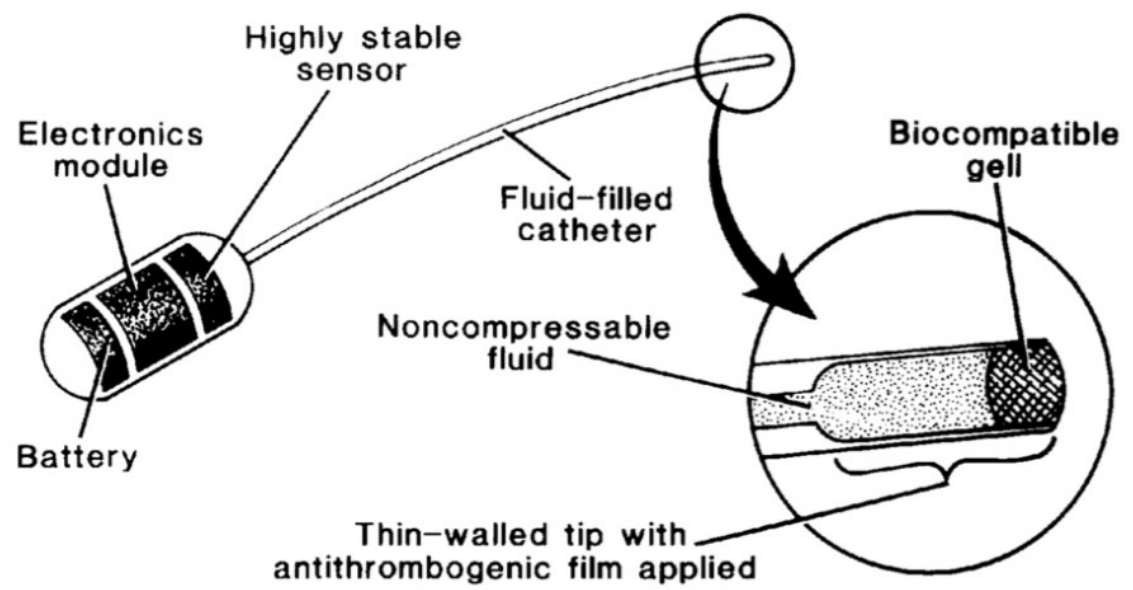

Figure 2.9: First solid state based implantable pressure system in 1989 [14, 15].

solutions still require external measurement equipment to provide stimulus to the MEMs sensor (note: capacitive based solutions can be read without any direct voltage or current stimulus [142]). MEMS catheter based pressure systems are very accurate however they allow for minimal overall movement of the test subject due to its direct connection with a larger measurement source. Similar to other direct methods, the surgical procedure can also cause possible complications such infection or trauma to the heart [198-202]. Several commercial catheter options are available for large and small animal from Transonic Scisense [196] and Millar Instruments [203] that are available down to the $1.2 \mathrm{~F}$ size to fit in a mouse left ventricle, Fig. 2.10. These catheters are used in acute hemodynamic studies today [175, 191, 200]. The catheter used in this thesis utilizes a MEMS based pressure sensor. More info on the specific catheter used is provided in Section 4.4.2

Implantable Pressure Measurement: From Citters and Franklin's first implantable transducer in 1965 [193], implantable pressure telemetry has advanced a long way. The first fully implantable system for animal research, mentioned above, was completed by Brockway et al. [14], Fig. 2.9, however, various aspects of implantable telemetry system research have existed for human and animal research prior to this [204-207]. When referring to implantable blood pressure telemetry, a pressure sensor, typically MEMS based, is attached to a processing 


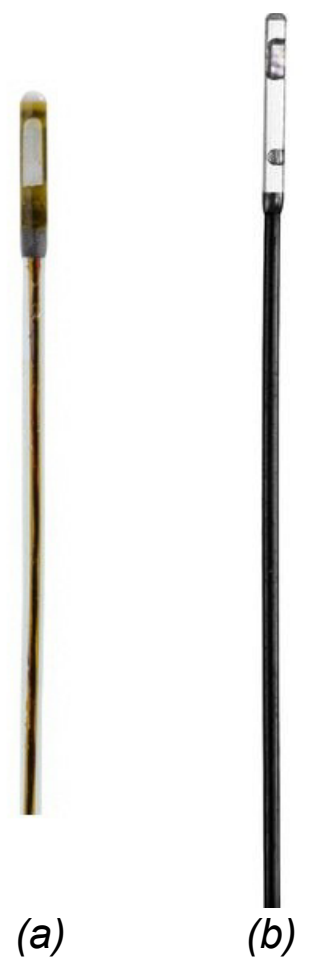

Figure 2.10: MEMS based pressure catheters for small animals from two manufactures: a) Millar Inc. and b) Transonic Scisense.

and control system where the data is transmitted external from the body, [197, 208]. Currently, implantable telemetry is considered the gold standard for large animal blood pressure measurement [168]. Various toxicology and pharmacology studies have been completed using implantable pressure telemetry [209.211].

Commercial pressure telemetry solutions exist for small and large animal research. Three main companies provide these systems: Millar Inc., Transonic Inc., and Data Science International (DSI).

Millar Instruments: Millar offers a single implant for the mice range [212] and 7 implants over its rat range [213]. In the mice range, the implant can only measure biopotential signals (ECG, EMG, EEG) and weighs $2.5 \mathrm{~g}$ with a volume of $1.4 \mathrm{~cm}^{3}$. These implants can only be used in animals larger than $22 \mathrm{~g}$. In the larger rat range, four types of signals can be measured with the available implants: pressure (arterial, venous, ventricular, intracranial), biopotential, tissue oxygen, and sympathetic nerve activity [213]. As the work in this thesis focuses on LV 


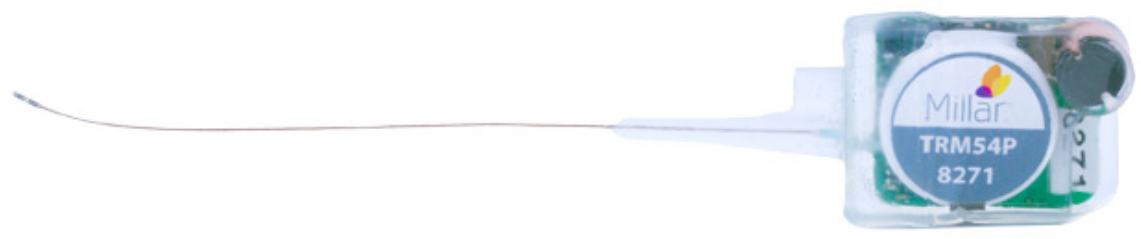

Figure 2.11: Millar TRM54P implantable pressure telemetry system [16]. Copyright (CMillar Instruments Inc.

blood pressure and volume monitoring, only single LV pressure implant, TRM54p [16], will be discussed for comparison. The TRM54p has a weight of $12 \mathrm{~g}$, a volume of $7 \mathrm{~cm}^{3}$, and a battery life of 4 to 7 h, Fig. 2.11. All Millar implants can be recharged via an external power station and are single use implants, i.e., cannot be interchanged with different sensors. Millar does not offer telemetry volume monitoring.

Transonic Inc.: Transonic Inc. offers two implants: EndoGear1 [17], Fig. 2.12a and EndoGear3 [18] Fig. 2.12p, large and small animal respectively. Unlike Millar's offerings, the EndoGear is interchangeable and configurable with various senors including doppler flow velocity, LV blood pressure, ECG, and temperature. Both the EndoGear 1 and 3 offer the same sensor options, however, for measuring LV pressure EndoGear3 can utilize a $1 \mathrm{~F}$ catheter size. Each system can be used with different batteries ranging from $2400 \mathrm{mAh}$ to $7200 \mathrm{mAh}$, providing 200 to 600 hours of use. The main difference between the EndoGear 1 and 3 are the size of the main implant and the ability to use wireless power. The EndoGear 1 is $6.35 \mathrm{~cm} \mathrm{x} 3.175 \mathrm{~cm}$ x $1.27 \mathrm{~cm}\left(25.60 \mathrm{~cm}^{3}\right)$ compared to the smaller EndoGear 3 at $2.8 \mathrm{~cm}$ x $3.0 \mathrm{~cm} \mathrm{x} 0.9 \mathrm{~cm}(7.5$ $\mathrm{cm}^{3}$ ). The EndoGear 3 can be powered wirelessly via an attached coil. Although, much larger than the Millar TRM54P, the EndoGear 3 is much more versatile in its measurement options. There is no implantable volume telemetry offered by Transonic Inc.

Data Science International: Data Science International (DSI) offers the most commercially available implantable telemetry systems for a variety of measurement signals (pressure, biopotential, glucose, temperature, heart rate, respiration) and across small, medium, and large an- 


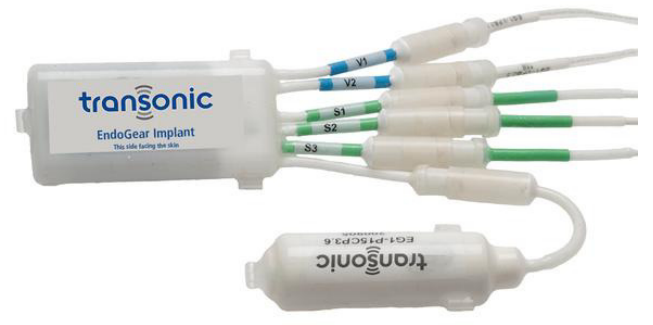

(a)

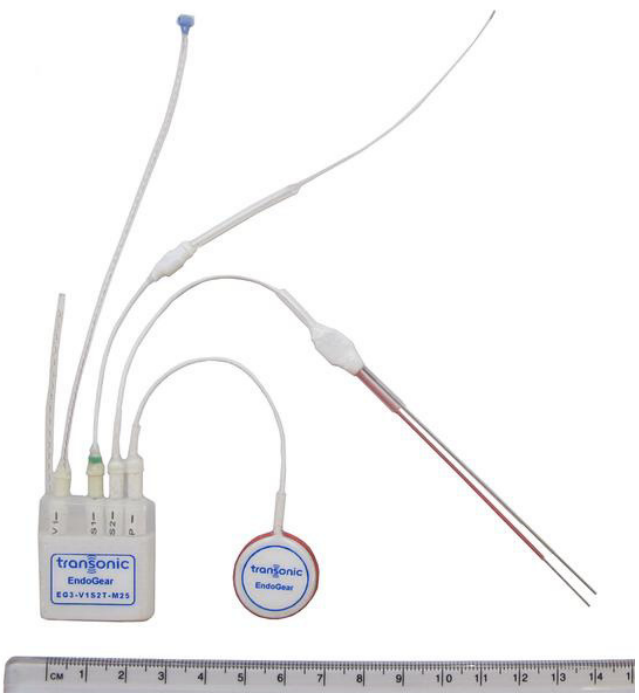

(b)

Figure 2.12: Transonic EndoGear for a) large animal EndoGear1 [17] and b) small animal EndoGear 3 [18]. Copyright (C)Transonic Inc.

imal ranges [214]. DSI's implants are also offered in different shapes and battery sizes to suite the researchers requirements. Only two systems offered measure LV pressure, DSI HDS21 [19] and L11 [20], for medium (rat) and large animal, respectively. The DSI HD-S21 is able to measure LV pressure, ECG, temperature and heart rate. It weighs $8 \mathrm{~g}$ with a volume of $5.9 \mathrm{~cm}^{3}$ and has a 2 month battery life, Fig. 2.13 . The large animal implant, L11, measures the same signals however, is much larger in size at $5.9 \mathrm{~cm} \times 3.8 \mathrm{~cm} \times 1.5 \mathrm{~cm}\left(33.63 \mathrm{~cm}^{3}\right)$ and has a weight of $56 \mathrm{~g}$, Fig. 2.14. The L11 has a battery life of 105 days. There is no implantable volume telemetry offered by DSI.

Academic Research Devices: More recently, researchers have focused on creating fully custom wireless implantable system with integrated blood pressure sensors [89, 127, 137, 192, 208210, 215, 216]. These systems range from creating a fully implantable cardiovascular pressure monitor with a medical stent [85] to a fully implantable blood pressure sensor for hypertonic patients [217]. Furthermore, much research has been placed on miniaturizing and improving the pressure sensor itself [197, 215, 218, 219].

The most recent research in this application area is in long term cardiac monitoring of small 


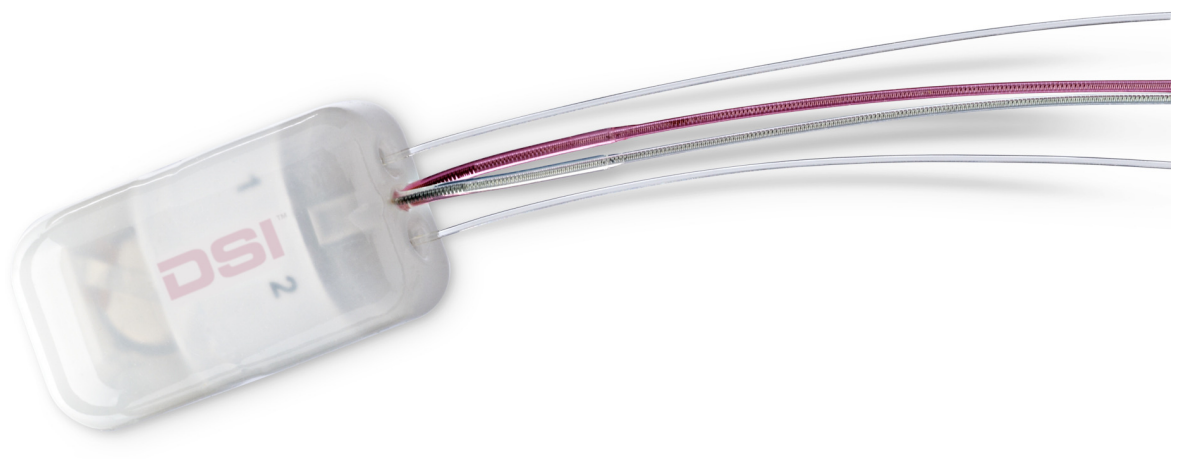

Figure 2.13: DSI HD-S21 small animal left ventricle pressure measurement system [19]. Copyright (C)DS.

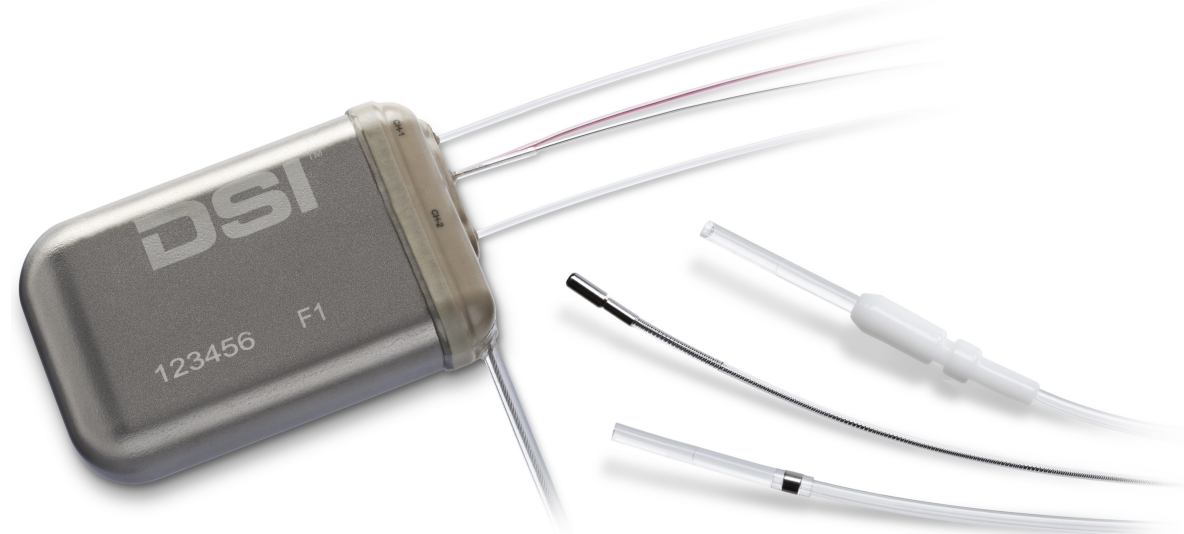

Figure 2.14: DSI L11 large animal left ventricle pressure measurement system [20]. Copyright (C)DSI. 


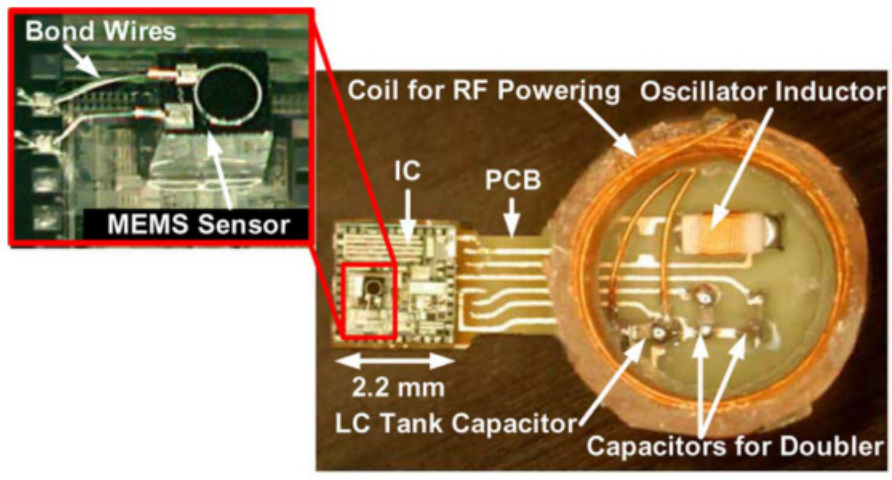

(a)

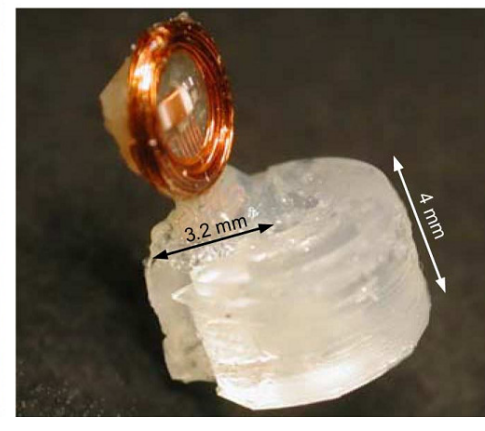

(b)

Figure 2.15: Implantable wireless telemetry system featuring a cuff blood pressure sensor: a) before packaging, b) after encapsulation, Cong [21]. Copyright (c)2010 IEEE.

animals. New research challenges occur such as reduced implantable area when dealing with smaller sized subjects over larger ones (e.g., non-human primates, humans, etc), as well as sensor drift and frequency resolution. This prevents larger external components from being used along with larger power sources. In [197], a pressure monitoring system is proposed that uses a passive telemetry link to send and receive power and data. Further advancement in miniaturization and packaging techniques has lead to the implantable system developed in [217]. In [89, 192], a fully implantable wireless pressure monitoring system was developed for hypertension patients. Here, both a custom pressure sensor and telemetry system were developed, however, it was designed to monitor femoral pressures and not the higher LV pressure. Others [127, 137] have focused on creating wireless power systems for these pressure implants to be used long term. Full implantable blood vessel cuff monitoring systems have also advanced throughout the years to current state-of-the-art methods such as the systems mentioned in [79] and [220].

In 2010, Cong [21] proposed a wireless batteryless implantable blood pressure monitoring microsystem for small laboratory animals, Fig. 2.15. Cong's implantable system employes a blood pressure cuff to measure the blood pressure around the blood vessel it is attached to. The pressure cuff is made from bio-compatible materials and contains an internal MEMS pressure 


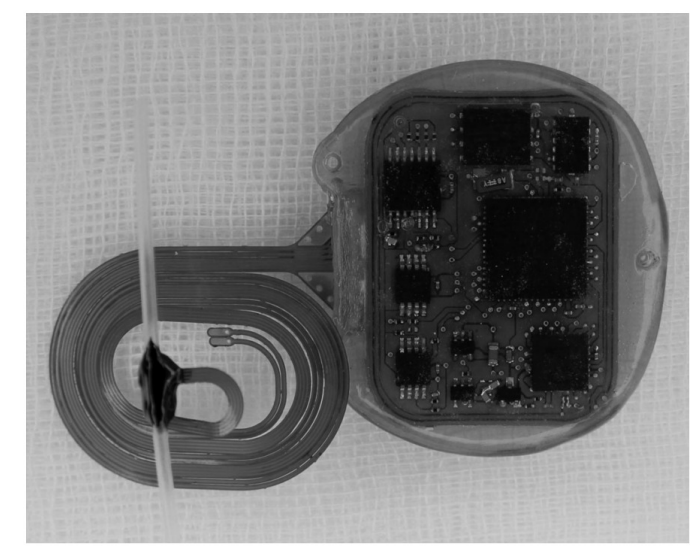

Figure 2.16: Implantable cardiac monitoring system developed by Volk [22] incorporating ECG, arterial pressure, and temperature sensors. Copyright (c)2015 IEEE.

sensor. The fully implantable system dissipates $300 \mu \mathrm{W}$ and was experimentally tested. The complete size of the wireless system is $6.4 \mathrm{~mm}$ diameter with a length of $4 \mathrm{~mm}$ and was also powered wirelessly via RF. However, Cong's implantable system is only focused on arterial blood pressure monitoring not LV blood pressure and does not combine any additional sensors to provide increased functionality.

A more recent implantable telemetry system was developed by Volk et al. [22] that measured ECG, arterial pressure, and temperature, Fig. 2.16, in mice using custom flexible ECG and pressure sensors. The system was powered wirelessly via Radio Frequency Identification (RFID) protocol, where a RFID reader is placed under the mouse cage to provide power and data transfer. This system was initially tested in-vivo in rats with successful results [22]. This system is much larger than the one presented in [21] at $29 \mathrm{~mm} \times 21.5 \mathrm{~mm}$. However, ECG and temperature monitoring are present. The increase in size is also attributed to the lower frequency RFID protocol used.

All telemetry systems mentioned in this section all measure blood pressure in one way or another, nevertheless, only a few (fully commercial solutions) measure the higher pressure higher bandwidth left ventricle signal. Furthermore, none of these existing systems measure blood volume. Implantable pressure-volume systems are discussed in the following section. 


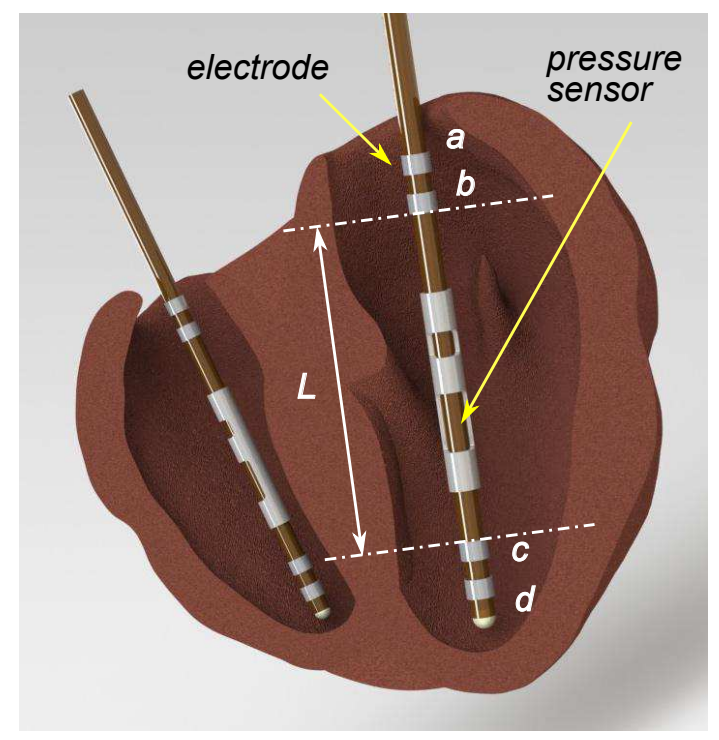

Figure 2.17: Example of the physical size of a implantable catheter, along with the electrode spacing for volume measurements and pressure sensor location.

\subsection{Blood Volume Measurement in Animals}

As described in Section 1.2, non-invasive left ventricle volume measurement methods were discussed indicating major issues including large equipment, highly trained personal, and large laboratories. In this section, direct volume measurement methods will be discussed in detail. Direct catheter based methods have been developed [77] [70] that provide a low cost, yet accurate measurement solution for finding LV volume in small test subjects. Two techniques exist to measure LV blood volume using a catheter based system: conductance and admittance. These techniques provide methods for the accurate measurement of LV blood volume from small lab animals in a cost effective and accurate fashion.

\subsubsection{Conductance}

The classical method of measuring blood volume in a catheter based system is the conductance technique, first introduced by Baan et al. in the early 1980s [77]. This method utilizes a tetrapolar catheter, Fig. 2.17, with two sets of rings located at the top and bottom of the catheter 
is placed inside the LV. The two outer electrodes inject a constant AC current into the blood to create an electric field within the LV and the two inner electrodes measure instantaneously the voltage created as the heart goes through its pump cycle. Conductance is calculated from the known excitation current and measured voltage. It should be noted that the myocardium conductance varies over the cardiac cycle.

The classical equation to convert the measured conductance to a LV volume is directly found by using Baan's linear conductance to volume equation (2.1):

$$
\begin{aligned}
\text { Volume } & =\frac{1}{\alpha} \rho L^{2}\left(G_{\text {meas }}-G_{m}\right) \\
& =\frac{1}{\alpha} \rho L^{2} G_{b}
\end{aligned}
$$

where $\rho$ is the blood resistivity $(\Omega m), \mathrm{L}$ is the length between the voltage sensing electrodes (m), $\frac{1}{\alpha}$ is the stroke volume (SV) calibration factor (originally assumed to be 1 ), $G_{\text {meas }}$ is the instantaneous measured conductance, $G_{m}$ is the surrounding myocardial conductance, and $G_{b}$ is the blood conductance [37].

Baan's equation makes the following assumptions: a) the electric field distribution is uniform throughout the cardiac cycle; b) the ventricular wall is insulated from the blood, i.e., the total measured conductance is due to the blood and not the heart muscle; c) the heart cavity has a cylindrical shape; and d) the catheter is stationary and always perfectly centred along the cylinder's axis.

The electrical model of the heart used in the conductance measurement method, Fig. 2.18 $\left(G_{b}\right.$ and $\left.G_{m}\right)$, models the blood and muscle as single resistive components. A single conductance measurement, $G_{\text {meas }}$, includes information about both blood, $G_{b}$ and myocardium muscle, $G_{m}$, leading to an over estimation of the LV blood volume. However, they are a combined value and cannot be separated without calibration [77]. Additionally, the model assumed that conductance $G_{m}$ remains constant throughout the cardiac cycle, this has since been addressed [221]. Based on these assumptions Baan suggested the use of a hypertonic saline bolus to be injected into the bloodstream in order to help estimate $G_{m}[37,222]$. Although this method is still used 


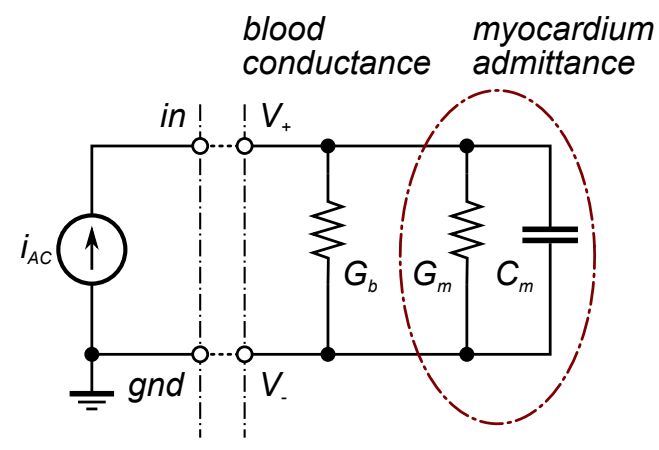

Figure 2.18: Conductance $\left(G_{b}\right.$ and $\left.G_{m}\right)$ and Admittance $\left(G_{b}, G_{m}\right.$, and $\left.C_{m}\right)$ electrical heart model, where $I_{\text {in }}$ represents the injected current, $G_{b}$ blood conductance, $G_{m}$ muscle conductance, and $C_{m}$ as the capacitive component of the muscle.

it has several significant drawbacks: it only provides one constant value for $G_{m}$ over the cardiac cycle, the saline bolus may temporarily change value of $G_{m}$, and the increased volume can cause harmful effects to the murine cardiovascular system [223, 224]. In addition, this technique is predominantly used in larger animals because the saline solution will significantly alter the blood resistivity and blood volume of smaller animals [70] causing Baan's equation to become invalid [77]. An alternative method to estimate parallel conductance based on the use of dual frequency stimulation was suggested in [225-227], which also does not account for the variation in $G_{m}$ during the cardiac cycle. However, these calibration techniques are not time dependent, therefore not creating instantaneous calibration to the conductance measurement. The conductance value requires a time dependent calibration factor to take care of the change in LV volumes (i.e., changing the electric field based on heart cycle) during the cardiac cycle from end-diastolic to end-systolic.

To correct for the effects from the non-uniform electric field on the conductance measurement, Wei et al. proposed a new improved equation denoted as Wei's equation [2.2] [228, 229].

$$
\begin{aligned}
\text { Volume } & =\frac{\gamma}{\gamma-G_{\text {meas }}-G_{m}} \rho L^{2}\left(G_{\text {meas }}-G_{m}\right) \\
& =\frac{\gamma}{\gamma-G_{b}} \rho L^{2}\left(G_{b}\right)
\end{aligned}
$$

Where $\rho$ is the blood resistivity $(\Omega m), \mathrm{L}$ is the length between the voltage sensing electrodes 
$(\mathrm{m}), \gamma$ is the field form factor, $G_{\text {meas }}$ is the instantaneous measured conductance $(\mathrm{S}), G_{m}$ is the surrounding myocardial conductance $(\mathrm{S})$, and $G_{b}$ is the blood conductance $(\mathrm{S})$ [228]. The field form factor $\gamma$ is calculated by (2.3).

$$
\begin{aligned}
\gamma & =\frac{-b \pm \sqrt{b^{2}-4 a c}}{2 a} \\
\text { where } a & =S V-\rho L^{2}\left(G_{b-E D}-G_{b-E S}\right) \\
b & =-S V\left(G_{b-E D}+G_{b-E S}\right) \\
c & =S V \times G_{b-E D} \times G_{b-E S} \\
S V & =\text { strokeVolume }(L) \\
\rho & =\text { blood resistivity }(\Omega m) \\
L & =\text { distance between sensing electrodes }(m)
\end{aligned}
$$

The main benefit of Wei's equation compared to Baan's is the compensation for the nonlinear electric field throughout the LV. This is corrected using the field form factor or $\gamma$. Wei's equation does however, require an independent stroke volume (SV) measurement for the calculation of $\gamma$. For larger subjects, Wei's equation can reduce the overestimation error to $10 \%$ compared to using the original Baan's equation [228]. In addition, this equation still requires parallel conductance removal through Hypertonic saline techniques. Therefore, a new technique, admittance, was created to separate blood volume from the surrounding muscle (myocardium) to calculate a more accurate LV volume measurement using Wei's equation. A full detailed analysis comparing the various conductance-to-volume equations is described in the following chapter.

\subsubsection{Admittance}

Porterfield et al. [230] proposed a new admittance based technique in the late 2000s, where the electrical and permittivity properties of the murine myocardium [231] are exploited to remove the myocardium conductance $\left(G_{m}\right)$ component from the overall measured signal. In addition, 
this technique includes the effects of the parallel myocardium capacitance $\left(C_{m}\right)$, where the original conductance method did not. This is described in the admittance equation (2.4), where the myocardium conductance and capacitance vary over the cardiac cycle.

$$
\begin{aligned}
Y_{\text {meas }} & =Y_{\text {blood }}+Y_{\text {muscle }} \\
& =G_{b}+G_{m}+j \omega C_{m}
\end{aligned}
$$

The known excitation current $i_{A C}$, whose frequency is set to $20 \mathrm{kHz}$, flows mostly through the blood and partially through myocardium volume. At that frequency, the blood exhibits purely resistive behaviour, while myocardium shows both resistive and capacitive properties [147, 149, 229, 231, 232]. Therefore, an updated electrical model of the heart is realized, Fig. $2.18\left(G_{b}, G_{m}\right.$, and $\left.C_{m}\right)$. Using an excitation frequency of $\sim 20 \mathrm{kHz}$, allows for the separation of the admittance of the myocardium from the combined measured admittance using electric field theory [230]. For this reason, in order to derive accurate blood volumetric results the measured conductance must be corrected for the parasitic effects of the myocardium.

The measured conductance and capacitance of the electric field are related by (2.5), where the ratio of $\frac{\sigma_{m}}{\varepsilon_{m}}$ (muscle conductivity and permittivity) is found using a surface probe measurement before the main experimental procedure. The derivation and process on measuring and calculating these parameters is detailed elsewhere [231].

$$
G_{m}=C_{m} \frac{\sigma_{m}}{\varepsilon_{m}}
$$

During an experimental procedure, only the magnitude and phase of the admittance signal will be measured (i.e., $\left.Y_{\text {meas }}=\left|Y_{\text {meas }}\right| \angle \theta_{Y}\right)$. This is conducted using the same catheter, Fig 2.17 , as the conductance method. Therefore, using these two parameters, the values of $G_{b}, G_{m}$, and $C_{m}$ can be calculated. Recall, that $Y_{\text {meas }}$ is given in eq. 2.4 and that blood is purely resistive and the myocardium has both resistive and capacitive electrical properties when using an excitation frequency of $20 \mathrm{kHz}$. With this knowledge, one can calculate the instantaneous values of $C_{m}, G_{m}$, and finally $G_{b}$ using equations $(2.5), 2.6$, and $(2.7)$, respectively. 


$$
\begin{gathered}
C_{m}=\frac{\left|Y_{\text {meas }}\right| \sin \left(\theta_{Y}\right)}{2 \pi f} \\
G_{b}=\left|Y_{\text {meas }}\right| \cos \left(\theta_{Y}\right)-G_{m}
\end{gathered}
$$

These three equations allow the parallel conductance (myocardium conductance) to be dynamically calculated based on the frequency dependent value of $C_{m}, 2.8$.

$$
\begin{aligned}
G_{b} & =\left|Y_{\text {meas }}\right| \cos \left(\theta_{Y}\right)-G_{m} \\
G_{b} & =\left|Y_{\text {meas }}\right| \cos \left(\theta_{Y}\right)-\frac{\sigma_{m}}{\varepsilon_{m}} * C_{m} \\
G_{b} & =\left|Y_{\text {meas }}\right| \cos \left(\theta_{Y}\right)-\frac{\sigma_{m}}{\varepsilon_{m}} * \frac{\left|Y_{\text {meas }}\right| \sin \left(\theta_{Y}\right)}{2 \pi f}
\end{aligned}
$$

This eliminates the need for hypertonic saline injection to calculate $G_{m}$, which is extremely beneficial for testing in small laboratory animals. Finally, with the complex admittance calculated (2.8), Wei's equation (2.2) can be used to calculate accurate volumes of the LV [231] [80]. State-of-the-art LV volume systems use this technique to calculate absolute volumes.

\subsubsection{Pressure-Volume Systems}

Implantable systems incorporating blood volume sensors for small animals have been an area that has yet to be exploited to its full potential. Currently, the majority of blood volume monitoring systems are what is known as tethered systems (i.e., attached to an end unit). These are generally commercially available from manufactures such as Transonic Scisense Inc. [3] and Millar Instruments Inc. [233]. These systems obtain their power and communication from attached end units, Fig. 1.4. The reason that these telemetry systems still remain wired is the increased complexity and external component count required when dealing with accurate blood volume measurements in comparison to simpler blood pressure measurements. By being tethered to an end unit the test subject is not allowed to move freely in their own natural envi- 


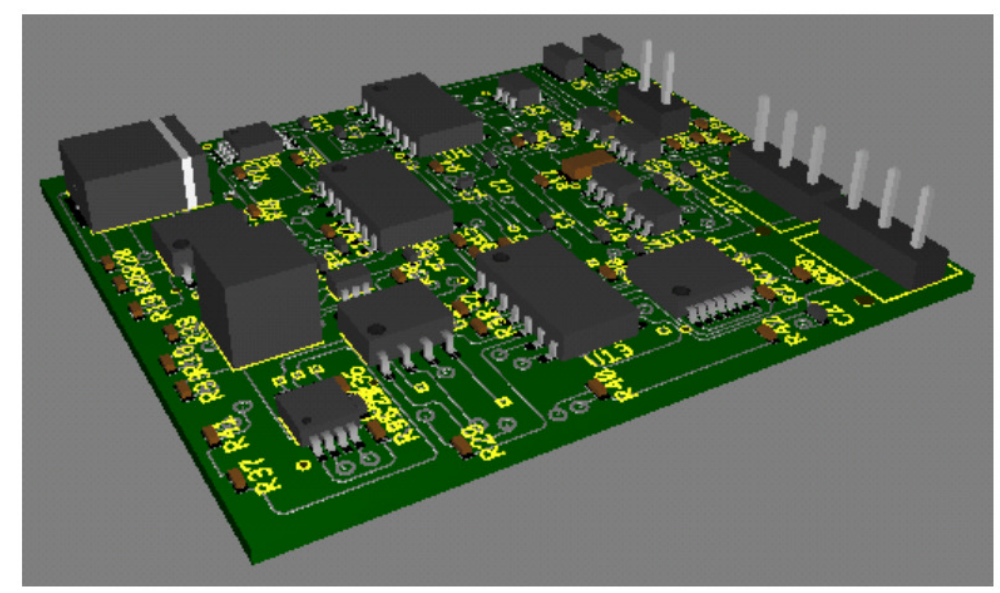

Figure 2.19: Implantable PV system developed by Raghavan [23] that is $17.67 \mathrm{~cm}^{2}$ and $27 \mathrm{~g}$.

ronment and may affect the measurement results [23]. The solution to this would be to create a wireless implantable system similar to implantable pressure systems described in the previous section but incorporate blood volume sensors as well.

One of the first developments of wireless implantable systems incorporating both LV blood pressure and volume sensors for small animal subjects reported by Uemura et al. [90] in 2004 was based on Bluetooth wireless technology. However, this telemetry system had a few drawbacks. First, the telemetry system was quite large as it used a programmable digital logic device and other large components to preform the processing. This increased the overall weight to 26 g. The second drawback of this initial telemetry system is that the conductance technique was used to measure volume rather than admittance and it also used a dual frequency approach to remove parallel capacitance from the measured volume. This reduced the accuracy of the volume measurements as is documented above.

Raghavan [23] improved this initial research by designing an admittance based telemetry system using analog circuit techniques, and a printed circuit board (PCB) whose size and weight are $17.67 \mathrm{~cm}^{2}$ and $27 \mathrm{~g}$, respectively, Fig. 2.19. This system improved over the previous system by using admittance based measurements rather than conductance methods, providing more accurate volume measurements. Although, improved over [90], there are still drawbacks and areas of improvement. This device was tested in large rats [23] and the size and weight of 


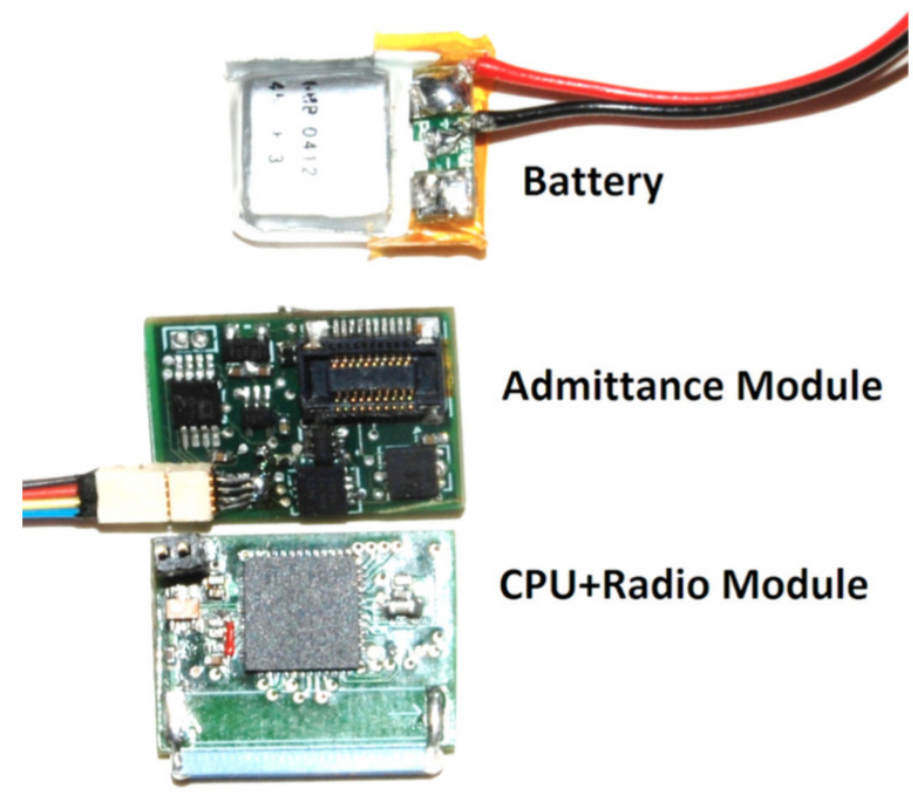

Figure 2.20: Implantable PV system developed by Schuhatovich [24] that weighs $4 \mathrm{~g}$ and has a volume of $10 \mathrm{~cm}^{3}$.

this telemetry system are still quite large for small animals, with the weight at $27 \mathrm{~g}$.

Schuhatovich [24] furthered Raghavan's research by reducing the size and weight to $4 \mathrm{~g}$ and $10 \mathrm{~cm}^{3}$, Fig. 2.20 . Schuhatovich's work mainly focused on the firmware development and built upon Raghavan's implant design by using a two board approach where each board measured $1.27 \mathrm{~cm} \times 1.9 \mathrm{~cm}$. The implant operated off of battery power and transmitted data on the $915 \mathrm{MHz}$ band. This work was only evaluated in-vitro without the use of phantoms or in animal models. It also had high operating currents of $7.6 \mathrm{~mA}$ in active mode and up to $38 \mathrm{~mA}$ in transmission.

More recently, Loeffler et al. [25, 26] designed an admittance based telemetry system with a volume of $5.92 \mathrm{~cm}^{3}$ and a weight of $7.2 \mathrm{~g}$, Fig. 2.21. This implementation is an extension of Raghavan's research and a second iteration of Schuhatovich's work [24,26]. In Loeffler's work, she decreased the transmission power and increased the weight compared to [24], however, her implementation was tested in large rats. As with [24] and unlike Raghavan [23], Loeffler's device operated in the $915 \mathrm{MHz}$ band.

Loeffler's work represents the most recent published work [25, 26] in implantable pressure- 


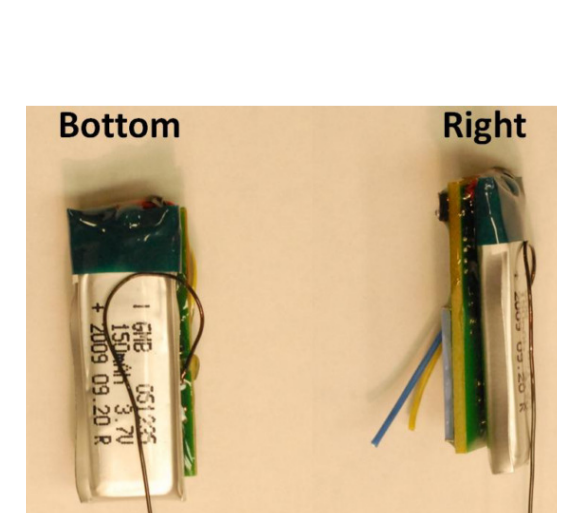

(a)

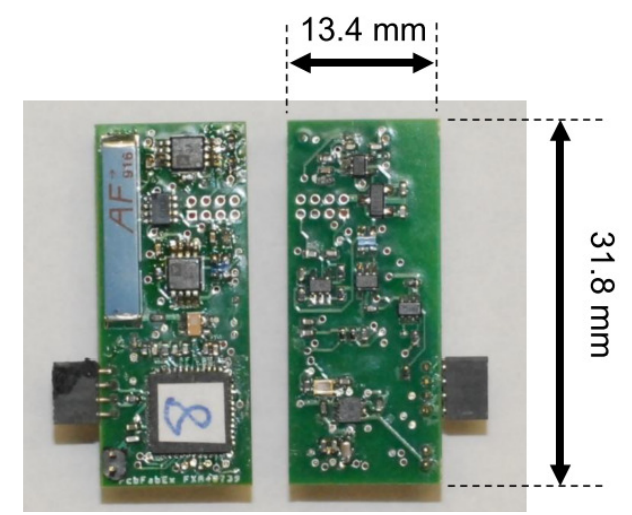

(b)

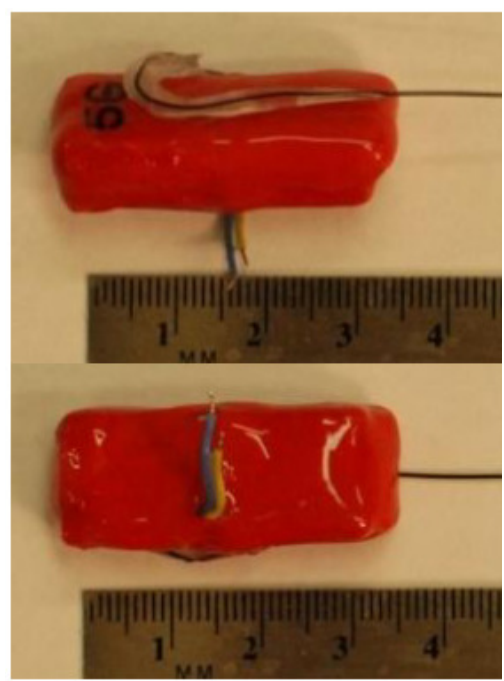

(c)

Figure 2.21: Implantable PV system developed by Loeffler [25,26] where a) shows the implant assembled with the battery; b) implant PCBs; and c) fully encapsulated implants. Implant is $5.92 \mathrm{~cm}^{3}$ in volume and has a weight of $7.2 \mathrm{~g}$. 


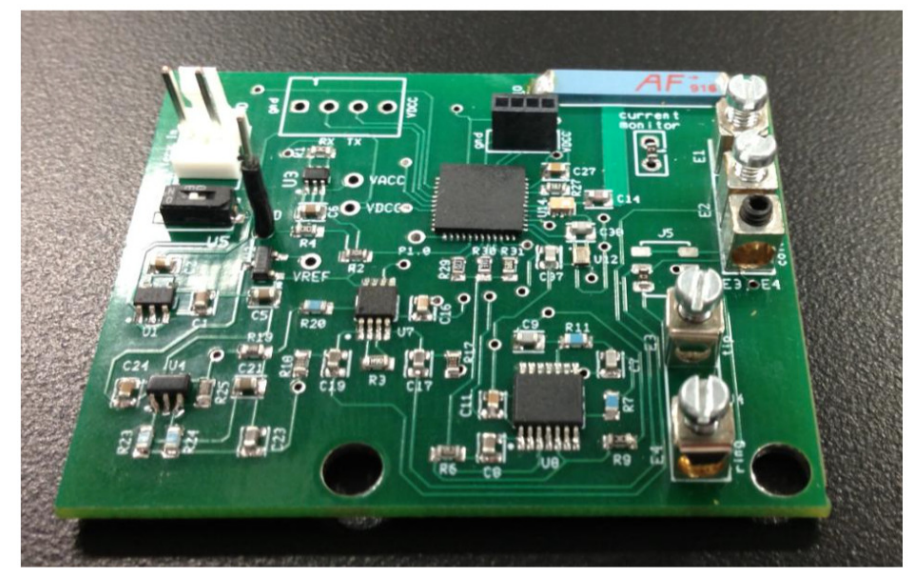

Figure 2.22: System designed by Holt [27] to measure volume in the right ventricle. Volume is $96.7 \mathrm{~cm}^{3}$.

volume telemetry systems for small animal cardiac research, however, more recent work has been completed by Holt [27] and Le [234] that focused on the development of much larger higher power devices for heart failure patients. In [27], Holt created a device that measured right ventricle blood volume only with future intentions for it to be used in conjunction with pacemakers. This device was $5 \mathrm{~cm}$ x $7.6 \mathrm{~cm}$ x $2.5 \mathrm{~cm}\left(96.7 \mathrm{~cm}^{3}\right)$ and used a $1000 \mathrm{mAh}$ battery, Fig. 2.22, This system did not contain any pressure monitoring and was not tested in-vivo.

Following the device in [27], Le developed a right ventricle volume measurement system [234]. This system measured volume, pressure (non-LV, surface pressure only), ECG, and temperature. It did not use a catheter to measure hemodynamic data, rather it uses a novel spring loaded device to perform measurements. Size and weight of this system was not reported, however, [234] focused on the development of the firmware rather than minimize size and power. The system operated on large 7.4 V $2200 \mathrm{mAh}$ batteries. This device was not tested in-vivo and did not include measurement of LV pressure or volume.

A full comparison of the relevant published works and the system designed in this thesis is shown in Table 6.7. If the telemetry system is to be implemented in a small animal subject, e.g., a rat and mouse, for an extended period of time without harm, further reduction in size, weight, and power consumption are required. 


\subsection{Conclusion}

In this chapter, a introduction into the field of implantable medical devices along with their various application areas and challenges is presented. A detailed discussion of blood pressure measurement technologies and techniques are presented with a focus on animal measurements. Relevant research and commercial blood pressure implants are discussed in detail outlining the few LV pressure systems available and the lack of commercial systems that measure LV blood volume. Moreover, an extensive background is provided for LV blood volume measurement techniques. Critical pressure-volume telemetry systems are discussed and compared, with the conclusion that further miniaturization is required for long term chronic use. 


\section{Chapter 3}

\section{Numerical Comparison and Calibration of Conductance Catheter Models*}

In the previous chapter, the focus was on implantable medical devices where we looked at different applications, sensors, and challenges involved in creating a implantable medical device. Furthermore, details of pressure-volume (PV) telemetry systems were discussed, with emphasis on the specific pressure and volume measurement approaches. More specifically, conductance and admittance measurement techniques were introduced followed by a brief introduction to the conductance-to-volume equations (Baan's, Wei's, and Dubois). These three conductance-to-volume equations are currently being used in industry yet there are no published works on their comparative analysis. In this chapter, a comparison study of all three conductance-to-volume equations along with the accuracy of the analytical model of the conductance catheter is presented. In addition, the overall accuracy of the admittance catheter volume measurements is studied and an empirical calibration factor to improve the volume measurement accuracy with the existing conductance-to-volume equations is presented. Although catheter design is not the direct focus of this thesis, it is important to study its effects on volume measurement in order to fully understand its application within a implantable envi-

*Portions of this chapter are published in IEEE Transactions on Biomedical Engineering [235]. 
ronment.

The rest of this chapter is organized as follows. In Section 3.1 the basic principle behind conductance based PV measurements with a detailed look at each conductance-to-volume equation is introduced, numerical verification of the models is presented in Section 3.2, which is followed by in-vitro experimental data in Section 3.3. Sensitivity due to physical variations of the catheters and correction factor is in Section 3.4. Concluding remarks for this chapter are in Section 3.5.

\subsection{Conductance Based PV Measurements}

In this section, the ideal conductance-to-volume conversion technique is reviewed followed by a detailed look at Baan, Wei, and Dubois' conductance-to-volume equations. Calibration techniques are also discussed.

\subsubsection{Review of Ideal Conductance-to-Volume Technique}

The principle of idealized volumetric measurements of a cylindrical cavity made of perfectly conductive material $\left(\sigma_{m} \rightarrow \infty\right)$ and filled in with non-perfect conductive fluid $\left(\sigma_{b}, \varepsilon_{r}\right)$ is illustrated in Fig. 3.1 and Table 3.1. The volume is calculated from measured conductance values of a tetra polar catheter centred inside the cylinder, where the four ring electrodes ( in $V_{+}, V_{-}$, gnd ) serve as the input and output terminals. Thus, the conductance of the blood and myocardium volume is calculated from the known excitation current $i_{A M}$ (which is injected between ( in, gnd ) terminals), and the measured potential difference (which is measured between $\left(V_{+}, V_{-}\right)$terminals) by using Ohm's law. In the idealized case of cylindrical volume, and perfectly symmetrical homogeneous electric filed $\vec{E}(r, \theta)$, it is trivial to calculate that real cylindrical volume as $V_{\text {real }}=\pi R^{2} H$ and measured reference volume as exactly $V_{\text {ref }}=\pi R^{2} L$, where $R$ is the cavity radius, $H$ is the cavity height, and $L$ is known distance between the two output electrodes. Therefore, real volume $V_{\text {real }}$ is effectively measured between the centres of 
Table 3.1: Physical dimensions of used catheters.

\begin{tabular}{c|c|c|c|c}
\hline Catheter & $a[\mathrm{~mm}]$ & $b[\mathrm{~mm}]$ & $L[\mathrm{~mm}]$ & $a_{0}[\mathrm{~mm}]$ \\
\hline $\mathbf{1 . 2 F}$ Nominal & 0.5000 & 0.5000 & 4.5000 & 0.2500 \\
\hline$\# \mathbf{1 9 0}$ & 0.4768 & 0.4914 & 4.4966 & 0.2000 \\
\hline$\# \mathbf{3 1 5}$ & 0.5146 & 0.4648 & 4.5436 & 0.2000 \\
\hline $\boldsymbol{\# 5 0 7}$ & 0.5243 & 0.5166 & 4.5648 & 0.2000 \\
\hline$\# \mathbf{5 8 7}$ & 0.5301 & 0.4718 & 4.5529 & 0.2000 \\
\hline $\boldsymbol{\# 5 9 0}$ & 0.4848 & 0.4852 & 4.5046 & 0.2000 \\
\hline $\boldsymbol{\# 1 2 - 8 7 0}$ & 0.4815 & 0.5110 & 4.4787 & 0.2000 \\
\hline $\boldsymbol{\# 1 2 - 1 1 4 6}$ & 0.5150 & 0.5110 & 4.4663 & 0.2000 \\
\hline $\boldsymbol{\# 1 2 - 1 1 4 7}$ & 0.4969 & 0.5277 & 4.5501 & 0.2000 \\
\hline
\end{tabular}

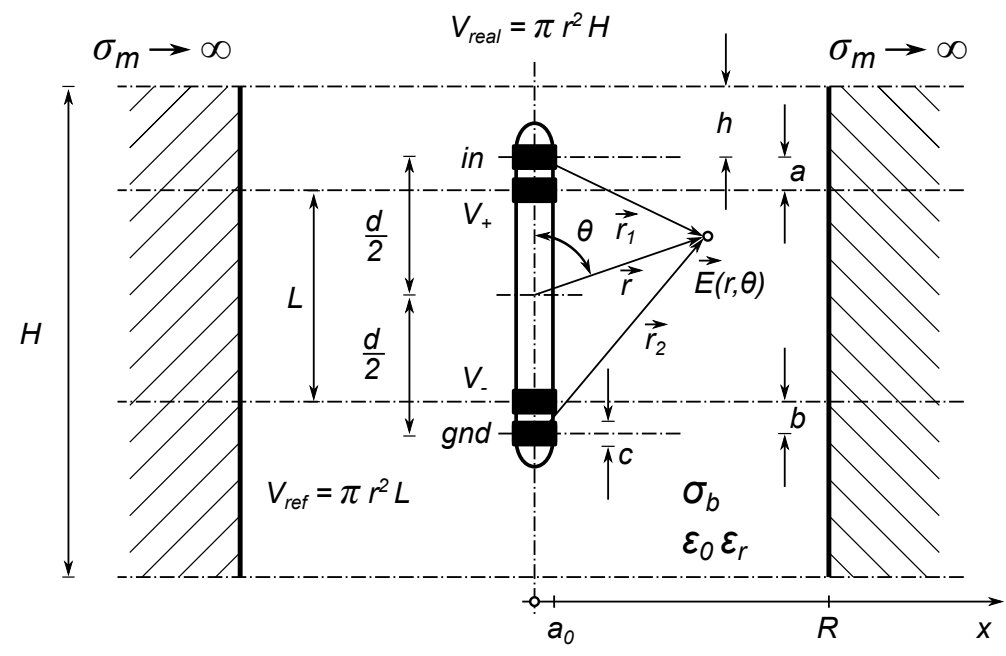

Figure 3.1: Geometry of a general conductance based measurement environment, where specific material properties can be modelled by $\sigma_{m}, \sigma_{b}, \epsilon_{r}, \epsilon_{m}$ and overall shape by R. Summary for eight catheter sizes used along with nominal $1.2 \mathrm{~F}$ is in Table 3.1 . 
$V_{+}$and $V_{-}$electrodes, which actually defines the smaller reference volume $V_{\text {ref. }}$ Obviously, the reference volume measurement is function of the catheter geometry; and the measured voltage between the two inner electrodes $\left(V_{+}, V_{-}\right)$is due to not only the conductance of the blood $\left(G_{b}\right)$ but also the parallel admittance of the myocardium $\left(G_{m}, C_{m}\right)$, Fig. 2.18.

\subsubsection{Conductance-to-Volume Equations}

Recall from the previous chapter, Baan's linear model [37] that connects measured conductance and volume $\mathrm{Vol}_{\mathrm{B}}$, (3.1). Note equation (3.1) and (2.1) are identical and only repeated for clarity purposes.

$$
\begin{aligned}
\mathrm{Vol}_{\mathrm{B}} & =\frac{1}{\alpha} \rho L^{2}\left(G_{\text {meas }}-G_{m}\right) \\
& =\frac{1}{\alpha} \rho L^{2} G_{b} \\
\alpha & =\rho L^{2} \frac{G_{b-E D}-G_{b-E S}}{S V}
\end{aligned}
$$

where, $\rho$ is blood resistivity, $G_{\text {meas }}$ is the measured conductance, $G_{m}$ is parallel conductance, $G_{b-E D}$ and $G_{b-E S}$ are the calculated blood conductance at diastole and systole respectively. This technique assumes a uniform electric field and a linear conductance-to-volume relationship, which results in the overestimated LV volume in mice. This overestimation is even greater in relative magnitude in larger animals due to the increased non-linearity of the conductance volume relationship for larger volumes [228].

In order to improve accuracy of [3.1], starting from Maxwell's equations in [228] Wei derived equation (3.4) from a cylinder model.

$$
\mathrm{Vol}_{\mathrm{W}}=\frac{\pi G_{\mathrm{inf}}^{2} L d^{2}}{4}\left[\frac{\frac{4 a_{0}^{2}}{d^{2}}+1}{\left(G_{\mathrm{inf}}-G_{b}\right)^{2}}-\frac{1}{G_{\mathrm{inf}}^{2}}\right]
$$


where, $G_{\text {inf }}$ is conductance of infinitely large volume of blood, assuming cylindrical blood volume; spherical electrodes; non-conducting inner electrodes $\left(V_{+}, V_{-}\right)$; and uniform electric field $\vec{E}(r, \theta)$. However, the shape of the ventricular is not cylindrical and $(3.4)$ does not include an empirical factor to force volume differences created from $G_{b_{-} \text {ES }}$ and $G_{b_{-} \text {ED }}$. Wei introduced an empirical correction factor $\beta$ to include the experimentally measured SV in the analytic equation (3.6] [228]. The improved analytic approximation becomes (3.5).

$$
\begin{aligned}
\operatorname{Vol}_{\mathrm{W}} & =\frac{\beta}{\left(G_{\text {inf }}-G_{b_{\text {ED }}}\right)^{2}}-\frac{\beta}{G_{\text {inf }}^{2}} \\
\text { where } \beta & =S V\left[\frac{1}{\left(G_{\text {inf }}-G_{b_{-E D}}\right)^{2}}-\frac{1}{\left(G_{\text {inf }}-G_{b_{-} \text {ED }}\right)^{2}}\right.
\end{aligned}
$$

From the analytic approximation equation (3.5), Wei's non-linear conductance to volume equation (3.6) is empirically developed for non cylindrical volumes which includes an empirical correction factor $\gamma$ to force the measured Stroke Volume(SV) to the known SV, measured from an independent SV probe.

$$
\begin{aligned}
\mathrm{Vol}_{\mathrm{W}} & =\frac{\gamma}{\gamma-G_{\text {meas }}-G_{m}} \rho L^{2}\left(G_{\text {meas }}-G_{m}\right) \\
& =\frac{\gamma}{\gamma-G_{b}} \rho L^{2}\left(G_{b}\right)
\end{aligned}
$$

where, $\rho$ is the blood resistivity $(\Omega \mathrm{m}), \mathrm{L}$ is the length between the voltage sensing electrodes $(\mathrm{m}), \gamma$ is the field form factor, $G_{\text {meas }}$ is the instantaneous measured conductance $(\mathrm{S}), G_{m}$ is the surrounding myocardial conductance $(\mathrm{S})$, and $G_{b}$ is the blood conductance $(\mathrm{S})$ [228]. The field form factor $\gamma$ is calculated by (3.7). Note (3.6) is identical to (2.2) and (3.7) is identical to (2.3) from chapter 2, it is repeated here for readability purposes only.

$$
\begin{aligned}
\gamma & =\frac{-b \pm \sqrt{b^{2}-4 a c}}{2 a} \\
\text { where } a & =S V-\rho L^{2}\left(G_{b-E D}-G_{b-E S}\right) \\
b & =-S V\left(G_{b-E D}+G_{b-E S}\right) \\
c & =S V \times G_{b-E D} \times G_{b-E S}
\end{aligned}
$$


In [228] it is indicated that (3.6) reduces volume calculation error by an order of magnitude relative to the error of Baan's equation (3.1).

$$
\mathrm{Vol}_{\mathrm{D}}=\pi a_{0}^{2} L-\frac{S V\left(Y-Y_{a 0}\right)\left(Y_{E D}-Y_{\mathrm{inf}}\right)^{2}\left(Y_{E S}-Y_{\mathrm{inf}}\right)^{2}\left(Y+Y_{a 0}-2 Y_{\mathrm{inf}}\right)}{\left(Y-Y_{\mathrm{inf}}\right)^{2}\left(Y_{a 0}-Y_{\mathrm{inf}}\right)^{2}\left(Y_{E D}-Y_{E S}\right)\left(2 Y_{\mathrm{inf}}-Y_{E D}-Y_{E S}\right)}
$$

As a further extension to (3.4) Dubois et al. recently developed a non-linear admittance-tovolume (3.8) that is computationally simpler than Wei's equation (3.6) (3.7) [80]. The equation is derived for the case of finite cylindrical volume and takes into account the small complex component of blood's admittance by including both blood conductivity $\left(\sigma_{b}\right)$ and blood permittivity $\left(\varepsilon_{b}\right)$. The equation uses the raw measured admittance $Y$ and eliminates the parallel contribution of the inherent myocardium $\left(\sigma_{m}+i \omega \varepsilon_{m}\right)$. The asymptotic admittance, $Y_{\text {inf }}$, is calculated purely analytically based on catheter geometry $L, d$ and the catheter radius $a_{0}$, Fig. 3.1 Admittances $Y_{E D}$ and $Y_{E S}$ are measured at diastole and systole respectively, while admittance $Y_{a 0}$ is calculated at the boundary condition where the radius of the cylinder is equal to the radius of the catheter. The equation is derived from the analytical derivation (3.4), while the authors imply it is self-calibrating for any effects of either the electrodes or catheter system overall. The mean error of $\mathrm{Vol}_{\mathrm{D}}$ compared to ultrasound measurements was reported to be within the industry acceptable range of 15\% [80]. In-vivo studies have also been completed, [33, 236] that compare measured volumes calculated using Wei's equation along with the admittance technique to MRI and 3D echo cardiography. Additional details on these studies is provided in Section 6.2.4

\subsubsection{Calibration Techniques}

All three equations (3.1) 3.6 and (3.8) require some form of empirical correction factor to compensate for the irregularity of the non-uniform myocardium, which must be determined in-vivo by using an independent stroke volume measurement, for instance a flow meter. The main purpose of in-vivo calibration is to estimate the parallel contribution of the myocardium, 
$G_{m}$ [237], while in-vitro calibration is required to correct the magnitude and phase angle of the measured admittance [229]. Magnitude calibration focuses the electrical instrumentation that the catheter is connected to, for example, known resistor and capacitor (RC) loads are used to calibrate the conversion from voltage to conductance. Phase calibration is performed by obtaining admittance measurements in Plexiglas cuvettes filled with saline of known conductivities that span the range of typical conductivity values for murine blood and myocardium [229]. The purpose of this calibration is to determine the phase shift attributed to the catheter itself (since saline has a negligible complex component in its admittance) so that the parasitic error is subtracted from the measured results. The specific calibration techniques used for the designed PV telemetry system in this thesis are found in Chapter 5 Section 5.3 .

\subsection{Numerical Comparison of Equations}

Using COMSOL Finite Element Method (FEM) simulation setup [238] as the reference, the accuracy of the three conductance-to-volume conversion equations were evaluated.

\subsubsection{FEM Numerical Conductance Model Setup}

To numerically evaluate the analytical conductance-to-volume model equation (3.4), the geometry of the catheter in the FEM simulation is modified to match that of the one used in the derivations in [228]. The catheter used in the derivation of (3.4) neglects the effects of the catheter body, and assumes that the input electrodes are perfect spheres of radius $a_{0}$ while the voltage electrodes are assumed point size in order to eliminate the averaging of voltage values of the entire electrode. This is the ideal case, in a realistic catheter the electrodes are platinum cylindrical rings rather than spheres and the measurement electrodes measure the average voltage induced from the electric field produced via an instrumentation amplifier circuit.

The geometry of the catheter model created follows that of Fig. 3.1 and the nominal 1.2F catheter dimensions outlined in Table 3.1. The catheter is centred in a cylindrical cuvette and 
modelled as a cylinder of radius $a_{0}$ and total length $8 \mathrm{~mm}$. The top of the source electrode (top) is located $2 \mathrm{~mm}$ below the surface of the cuvette with the remaining dimensions of the $1.2 \mathrm{~F}$ (Table 3.1 measured centre to centre. Distortions of the electric field due to proximity effects from the surface of the cuvette should not be present since the catheter is inserted deeper than $0.5 \mathrm{~mm}$ into the cuvette [238]. The cuvette radius is parameterized along with its $20 \mathrm{~mm}$ length to vary the volume of the cuvette cylinder between $8 \mu \mathrm{L}$ and $60 \mu \mathrm{L}$ which corresponds to the typical range of murine LV volumes. For example, the shape and distribution of the resulting electric field for a single volume is shown in Fig. 3.2. This simulated geometry is referred to as the standard geometry.

The cuvette material is set to have a conductivity of $1.26 \mathrm{~s} / \mathrm{m}$ and a relative permittivity of 86 to replicate the electric properties of $0.1 \mathrm{~mol}$ Potassium Chloride (KCL) solution in 1 litre of water [228]. The KCL solution is used as a non-perfect conductive solution to measure conductances corresponding to known volumes. The material of all four electrodes is set to a conductivity of 9,661,000 s/m (Platinum). The boundary AC current source at the cylindrical surface of the source electrode is set to a current density of $9.5 \mathrm{~A} / \mathrm{m}^{2}$, equivalent to a peak current of $30 \mu \mathrm{A}$ at $10 \mathrm{kHz}$ (the equivalent electrical diagram is in Fig 2.18). It should be noted that in a realistic catheter, electrode edge effects result in higher current densities at the top and bottom of the electrode edges.

In order to represent the realistic case of current flow between the sensor itself and the instrumentation amplifier, the sensing electrodes are modelled as high impedance. In the ideal derivation of (3.4) no current flows into the sensing electrodes.

\subsubsection{Analytical Conductance-to-Volume Model Accuracy}

Three additional simulations are preformed to investigate the effects of the physical simplification of the analytical model (3.4) specifically with the sensing electrodes [228]; a) cylindrical sensing electrodes are changed to spherical electrodes to match the physical model used in the derivation of (3.4), b) spherical sensing electrodes are set to $10^{\mu \mathrm{S}} / \mathrm{m}$ to approximate a non- 


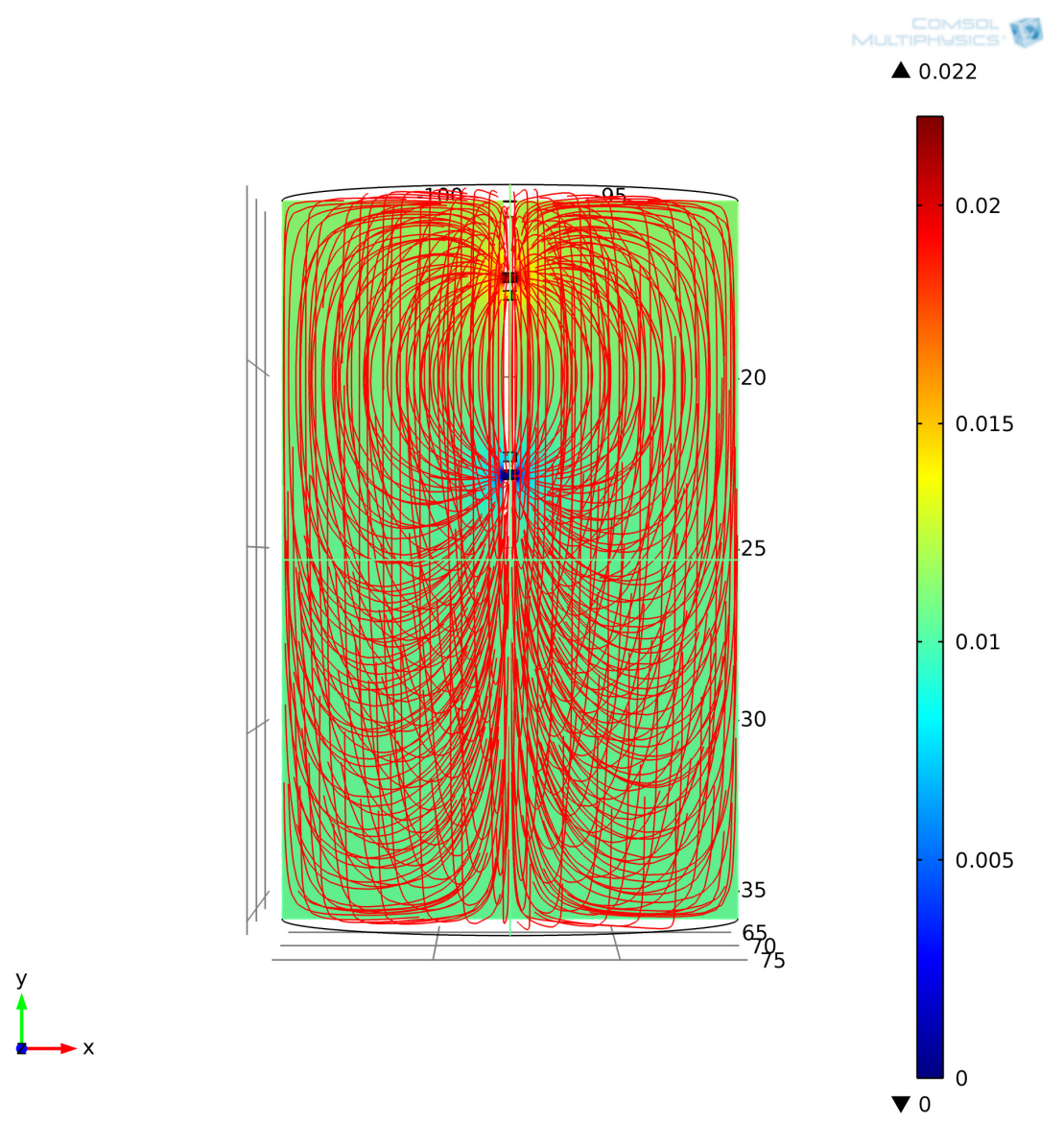

Figure 3.2: COMSOL FEM simulation of a standard geometry catheter in the standard environment. 
conductive material, and c) spherical electrodes are replaced by point probes to determine the effect of the shape and size of the electrode itself.

For each simulation, the measured voltage sensed from the inner electrodes is used to calculate the conductance values and the corresponding volume is calculated by (3.4). It can be shown that intermediate result for equation (3.4) includes an analytical expression for blood conductance $G_{b}$, which is found to be (3.9).

$$
\begin{aligned}
G_{b}= & \frac{\pi d\left(d^{2}-L^{2}\right)}{4 L} \times(\sigma+j \omega \varepsilon) \times \\
& \times\left[\frac{1}{\sqrt{a_{0}^{2}+\left(\frac{d}{2}\right)^{2}}}-\frac{1}{\sqrt{R^{2}+\left(\frac{d}{2}\right)^{2}}}\right]
\end{aligned}
$$

This value of $G_{b}$ is used to calculate an ideal conductance value from the known cuvette geometry, denoted as analytically calculated reference data. Four simulations are preformed with volumes ranging from $13.56 \mu$ to $435 \mu \mathrm{l}$ along with the analytically calculated reference conductances: a) analytically calculated reference $\mathrm{G}_{b}$ from known volumes $(3.9)$, b) original cylindrical environment described in Section 3.2.1, c) spherical electrodes low conductance, d) spherical electrodes modelled as in derivation of (3.4), and e) point probes, Fig 3.3, that are based on known cuvette volumes yielding five conductance-to-volume relationships. The full list of measured conductance values used to generate Fig. 3.3 and shown in Table 3.2 .

It becomes clear that the analytical equation greatly underestimates conductance values by an average of $135 \%$ when spherical electrodes are used to calculate conductance (i.e., comparing spherical electrodes, Fig. 3.3p, to analytically calculated conductance values Fig. 3.3a. This is the moel used in (3.4) and Furthermore, the analytically derived conductance values from (3.9) are not linearly correlated with either the cylindrical electrode simulated data or any set of the spherical electrode simulated data. It is shown, Fig. 3.3 , that when using the standard 


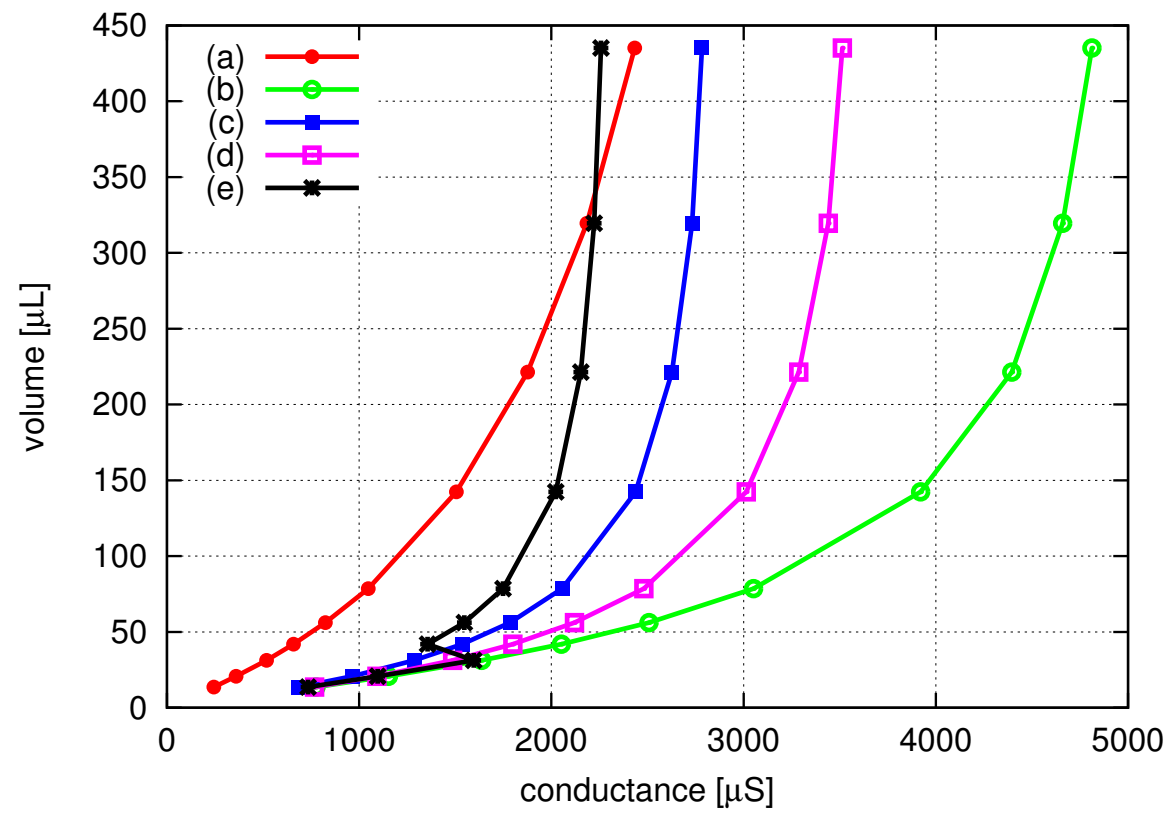

Figure 3.3: Conductance-volume data sets: a) Analytically calculated $G_{b}$ from known volumes (3.9), b) Simulated cylindrical electrodes, c) Spherical low conductance electrodes, d) Simulated spherical electrodes, e) Spherical low conductance point probes; are compared to analytical data for 3.9).

Table 3.2: Data sets used to generate Fig. 3.3 Conductance-volume data sets: a) Analytically calculated $G_{b}$ from known volumes (3.9), b) Simulated cylindrical electrodes, c) Spherical low conductance electrodes, d) Simulated spherical electrodes, e) Spherical low conductance point probes; are compared to analytical data for (3.9).

\begin{tabular}{l|l|l|l|l|l}
\hline $\begin{array}{l}\text { Known Volume } \\
{[\mu \mathrm{L}]}\end{array}$ & $\begin{array}{l}\text { Analytically } \\
\text { Calculated } \mathbf{G}_{b}[\mu \mathrm{S}]\end{array}$ & $\begin{array}{l}\text { Cylinder } \\
\text { Electrodes }[\mu \mathrm{S}]\end{array}$ & $\begin{array}{l}\text { Spherical } \\
\text { Low Conductance }[\mu \mathrm{S}]\end{array}$ & $\begin{array}{l}\text { Spherical } \\
\text { Electrodes }[\mu \mathrm{S}]\end{array}$ & $\begin{array}{l}\text { Spherical } \\
\text { Point Probes }[\mu \mathrm{S}]\end{array}$ \\
\hline 13.56 & 243.30 & 775.20 & 686.00 & 768.53 & 733.86 \\
\hline 20.64 & 360.03 & 1151.74 & 963.05 & 1092.76 & 1096.02 \\
\hline 31.27 & 518.00 & 1637.02 & 1286.59 & 1485.87 & 1596.21 \\
\hline 41.90 & 658.73 & 2052.26 & 1536.25 & 1799.02 & 1355.27 \\
\hline 56.08 & 824.50 & 2508.52 & 1784.84 & 2120.23 & 1547.56 \\
\hline 78.52 & 1046.99 & 3051.81 & 2055.13 & 2481.04 & 1748.81 \\
\hline 142.30 & 1505.87 & 3921.44 & 2437.91 & 3014.33 & 2022.66 \\
\hline 221.43 & 1876.91 & 4395.52 & 2627.12 & 3286.83 & 2152.78 \\
\hline 319.46 & 2184.25 & 4659.81 & 2731.91 & 3440.42 & 2222.64 \\
\hline 435.21 & 2433.46 & 4812.09 & 2783.55 & 3514.32 & 2258.44 \\
\hline
\end{tabular}


geometry (i.e., cylindrical electrodes that are a better representation of a realistic catheter) the average error is $177 \%$, Fig. 3.3 . The measured conductance values from the spherical data are offset from the analytically calculated data by a significant amount over the original cylinder electrode model however, this offset is more prominent for larger volumes. The offset decreases in magnitude when the inner sensing electrodes are simulated as non-conductive spheres and decreases further when the inner voltage probes are set to points inside the COMSOL environment. The simulation with spherical low conductance electrodes and point voltage probes produces the closest conductance values compared to the analytical data, which is expected because the analytical model assumed infinite volume, i.e. non-distorted field lines.

Overall this result indicates that the simplifying assumptions (e.g., spherical vs. cylindrical electrodes) made in the analytical derivation have significant effects on equations (3.4) and (3.9) accuracy. There are several theoretical reasons to possibly explain this behaviour: the electric potential is unevenly distributed on the surface of the electrodes, there is an interaction within the top pair of electrodes and within the bottom pair of electrodes, and finally the constraining volume of the cuvette has an effect on the generated electric field. These effects are unaccounted for in the analytical derivation. Therefore, the analytical solution (3.4) is too simplistic to accurately model a conductance-to-volume catheter.

\subsubsection{Comparison of Conductance-to-Volume Equations}

In the standard simulated geometry and simulation environment (cylindrical electrodes, 1.2F standard dimensions) each of the three equations is used to calculate volumes based on the measured conductance values. To simulate the asymptotic conductance, $G_{\text {inf }}$, the cuvette volume is set to $30 \mathrm{~mL}$, which resulted in the conductance value within $6 \%$ of the $G_{\text {inf }}$ in [228]. Since there is no muscle component in the simulation the $Y_{a 0}$ in Dubois' equation is set to zero, and it uses conductance values since the complex component is negligible. The resulting simulated conductance vs calculated volume for each equation is plotted in Fig. 3.4 along with the reference data set (simulated data). 


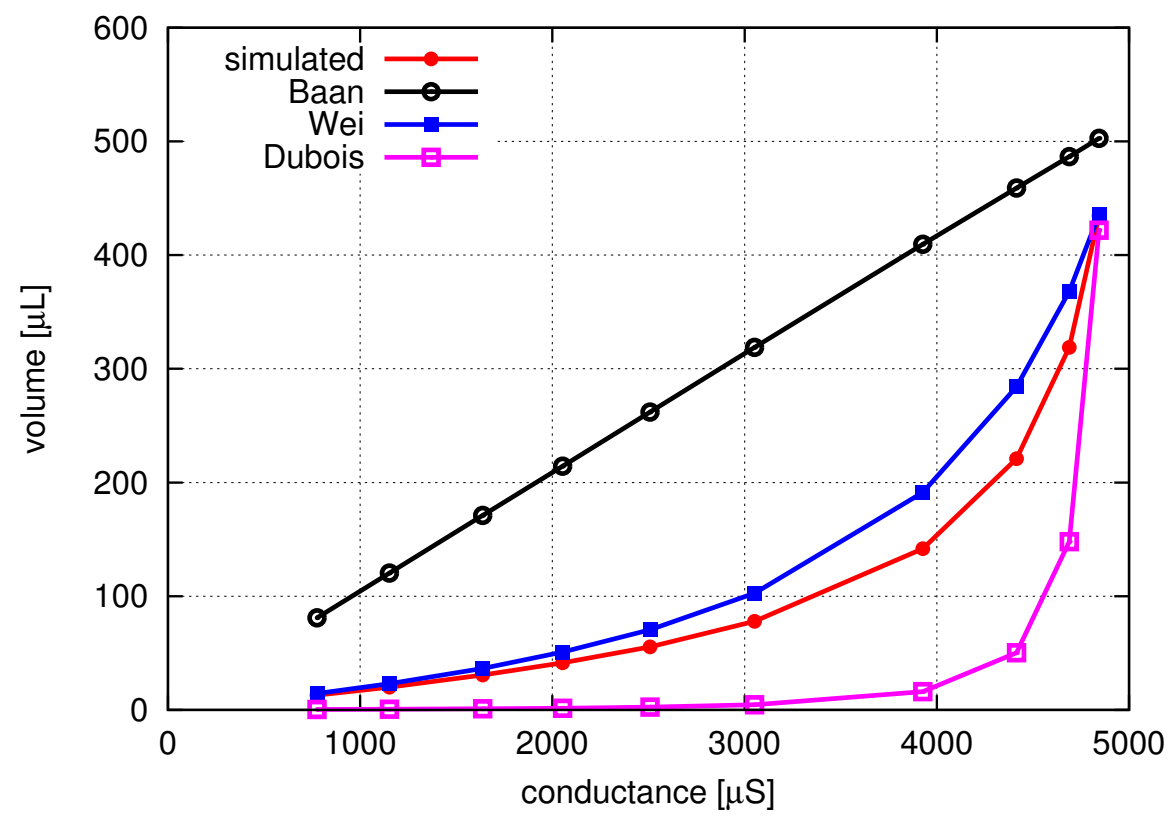

Figure 3.4: Comparison of calculated volumes from simulated data.

The volume range extends beyond the typical murine LV volume range to test the accuracy of the equations in the non-linear section of the conductance-volume relationship. Although these data is obtained from a simulated mouse catheter that would not measure this volume range in practice it is reasonable to use this data in comparing the conductance-volume equations since measurements from larger catheters (large animal) exhibit the same nonlinear relationship [228]. In this way each respective equation is tested more rigorously and the conclusions can be extended to the use of larger animal catheters as well. Baan's linear equation (3.1) consistently overestimates volumes over the entire conductance range, on average $295 \%$, Table 3.3. This is in accordance with Wei's results [228] and is explained by the fact that Baan's linear equation (3.1) cannot model the non-linear relationship at higher volumes.

Dubois' equation (3.8) consistently underestimates volumes, on average 80\%, throughout the conductance range. When (3.8) is used to calculate volumes with conductance values generated from (3.9), Fig. 3.3 dataset (a), the calculated volumes match the reference volumes well, average error less than 1\%. This suggests that Dubois' equation (3.8) is a rearranged version of the analytical equation (3.4) derived from Maxwell's equations [228], which by itself 
Table 3.3: Comparison between known cuvette volumes (simulated) and volumes calculated using (3.1), (3.4), and (3.8). Graphical representation Fig. 3.4.

\begin{tabular}{c|c|c|c}
\hline Known Volumes $[\mu 1]$ & Baan $[3.1][\mu l]$ & Wei 3.6$][\mu l]$ & Dubois $[3.8)[\mu l]$ \\
\hline 12.99 & 80.97 & 14.36 & 0.33 \\
\hline 20.08 & 120.30 & 23.05 & 0.56 \\
\hline 30.71 & 170.99 & 36.53 & 0.98 \\
\hline 41.34 & 214.36 & 50.81 & 1.52 \\
\hline 55.51 & 262.02 & 70.58 & 2.45 \\
\hline 77.95 & 318.76 & 102.55 & 4.48 \\
\hline 141.73 & 409.60 & 191.19 & 16.02 \\
\hline 220.87 & 459.11 & 284.18 & 50.31 \\
\hline 318.9 & 486.72 & 368.18 & 147.98 \\
\hline 434.65 & 502.62 & 436.02 & 421.98 \\
\hline \hline Avg error $[\%]$ & 294 & 21 & 80 \\
\hline
\end{tabular}

we showed to be oversimplified and inaccurate to model the conductance-to-volume relationship for realistic catheters, Section 3.2.2. Consequently, Dubois' equation is also determined to be inaccurate in modelling the conductance-to-volume relationship for cylindrical cuvette volumes. Volumes calculated using Wei's equation (3.6) are overestimated and have an average error of $21 \%$ relative to the reference volumes yet they are the most accurate compared to those obtained using the other equations. The error is greatest for mid-range conductance values since Wei's equation also uses SV to force the calculated volumes to match the ESV and EDV. Plotting volumes calculated using Wei's equation versus the reference volumes displays a strong linear correlation with an $\mathrm{R}^{2}$ value of 0.98 and a slope of 0.98 , Fig. 3.5. This correlation is the most linear and has a slope with a value closest to unity when compared to similar plots of volumes calculated using either Dubois' or Baan's equation versus reference volume, Fig. 3.5 This suggests that Wei's equation (3.6) is the most accurate conductance-volume equation and should be preferred over Baan's or Dubois'. Furthermore, the linear correlation suggests that the error in Wei's equation can be calibrated for by subtracting a calibration constant from all the calculated volumes. 


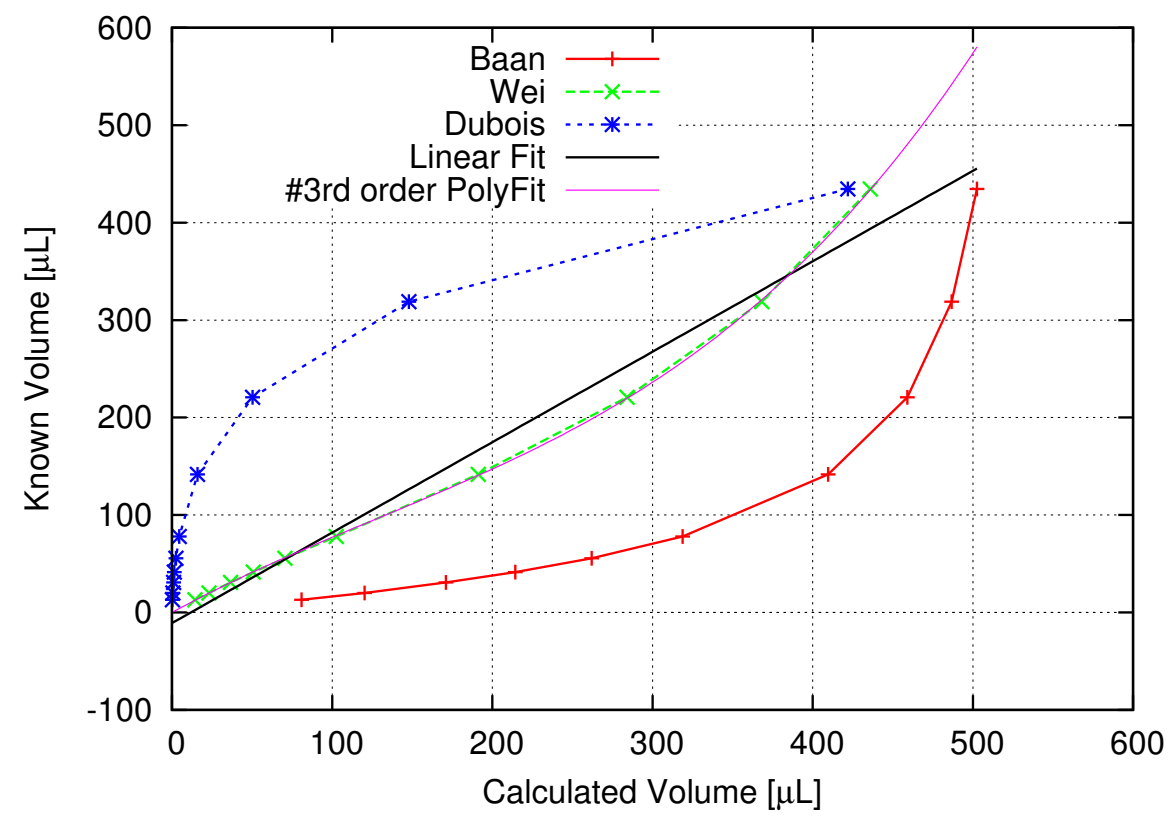

Figure 3.5: Comparison conductance to volume equations vs known cylindrical volumes. Ideal relationship should be linear.

\subsection{In Vitro Catheter Experiments}

In-vitro catheter measurement experiments were conducted with available industry catheters to further analyze the accuracy of Wei's conductance-to-volume equation (3.6). In addition, the sensitivity of the electrode geometry was investigated to determine its impact on volume measurements.

\subsubsection{In-Vitro Conductance Catheter Experimental Setup}

For the in-vitro measurement setup a radially organized cuvette tray with an integrated guide was designed, Fig. 3.6, which enabled the to elimination of variations in the catheter's position within the cuvette and, therefore, reduce effect on measured conductance [238]. The set of cuvettes span a volume range of approximately $15 \mu \mathrm{L}$ to $600 \mu \mathrm{L}$ and can be used with either mouse or rat catheters. The entire apparatus is constructed out of Plexiglas and the cuvette dimensions are measured to within less then $12.7 \mu \mathrm{m}$ and the volume is calculated to within 


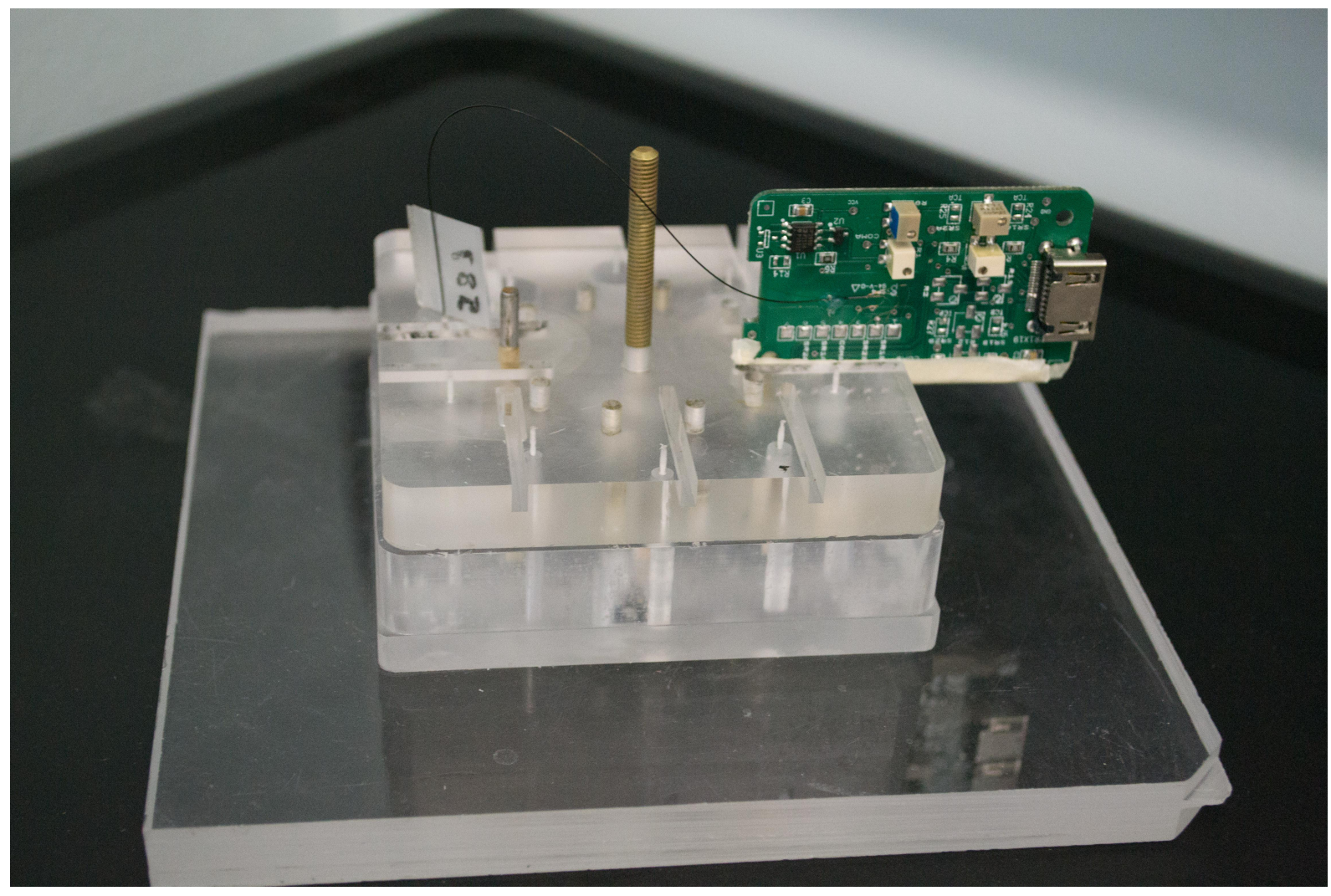

Figure 3.6: Radially organized cuvette tray with an integrated guide for various cylindrical volume etalons.

$0.21 \mu \mathrm{L}$ in the murine $\mathrm{LV}$ volume range and to within $0.6 \mu \mathrm{L}$ in the rat $\mathrm{LV}$ volume range.

Eight custom Transonic Scisense Inc. 1.2F catheters are used in this experiment, five catheters are manufactured without a pressure sensor (volume only), serial numbers: 190, $315,507,587,590$ and three with a pressure sensor, serial numbers 12-870, 12-1146, $12-$ 1147. A microscope with digital image capture capabilities is used to measure the complete catheter geometry $\left(a, b, d, L, a_{0}\right)$, Fig. 3.7, full dimensions are summarized in Table 3.1, while the peak output current of the catheters is measured as $24.04 \mu \mathrm{A}$ at a frequency of $20 \mathrm{kHz}$. The conductivity of the KCL solution is $0.77 \mathrm{~s} / \mathrm{m}$. All catheters are limited to the same depth of approximately $4 \mathrm{~mm}$ when inserted into the guide to ensure minimal field distortion.

The conductance measurements are recorded using an oscilloscope that interfaces with a Transonic Scisense Advantage ADV500 system [3]. The Advantage system interfaces with the catheter and outputs the raw admittance signal encoded as two output channels; one for mag- 


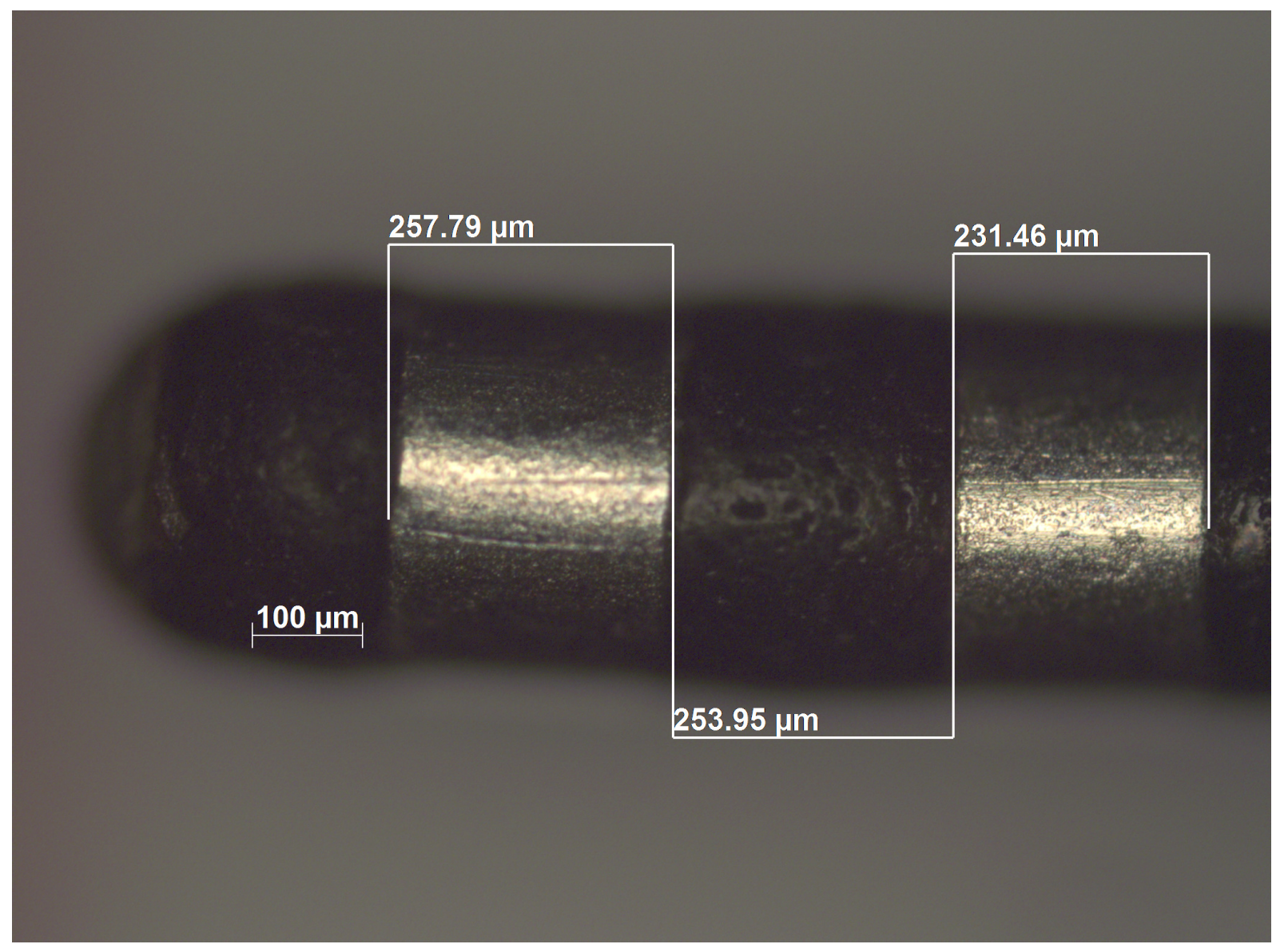

Figure 3.7: Catheter 190 under microscope measuring source electrode rings. 


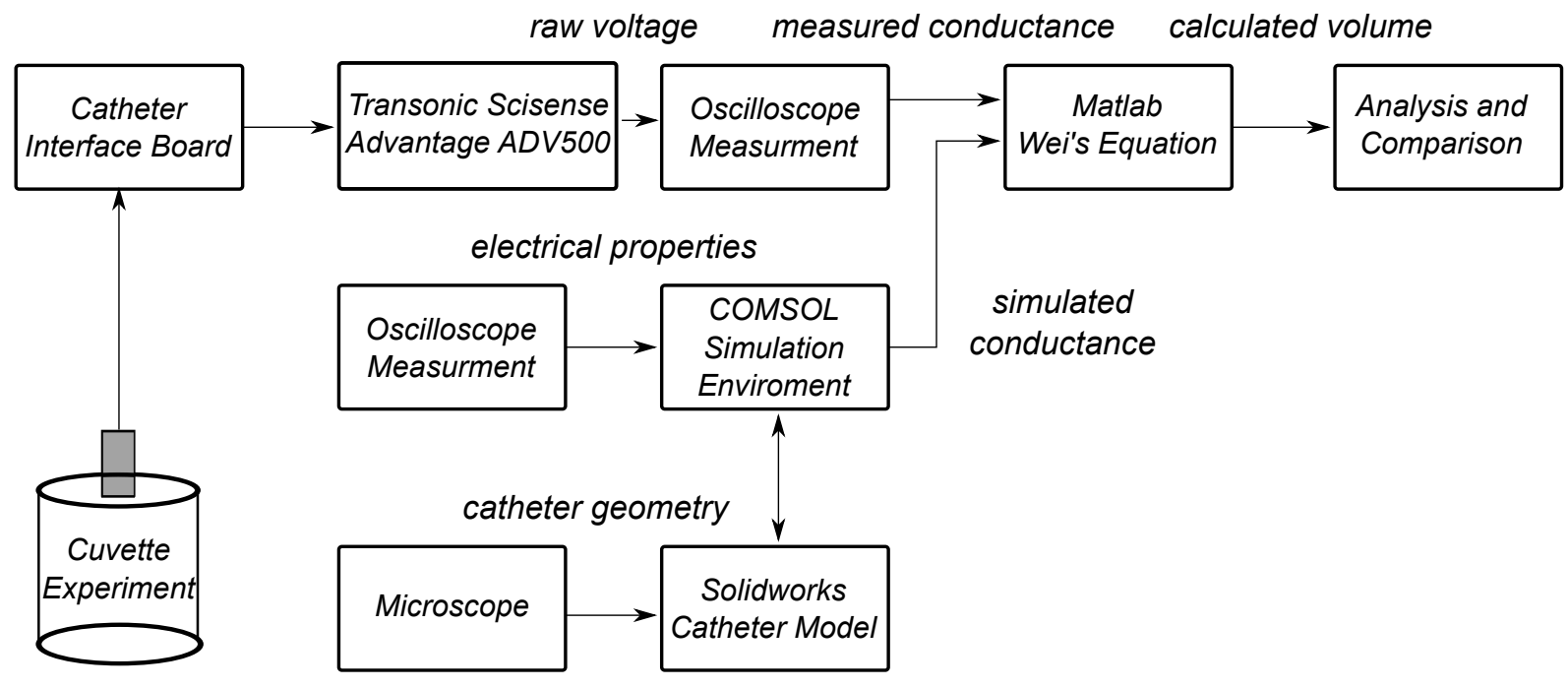

Figure 3.8: Experimental and comparison methodology flow.

nitude and one for phase. The phase is negligible as is expected due to the fact that Plexiglas has a very low permittivity and the KCL solution should produce no phase shift.

The measurement methodology outlined in Fig. 3.8 is followed for each catheter, where conductance values are measured from each cuvette shown in Fig. 3.6. Each cuvette volume is measured four times with the average conductance value used in the calculation of (3.6). In addition to the in-vitro measured data, COMSOL simulations are also completed with the respective electrode dimensions, KCL solution conductivity, peak output and frequency current of the source electrode. Together, these are referred to as the measured geometry and measured environment.

\subsubsection{In-Vitro Conductance Catheter Experimental Results}

In-vitro conductance measurements from each catheter are compared to the known volumes and summarized in Fig. 3.9. In addition, the simulated conductance for each catheter model is plotted against the measured conductance for the corresponding catheter, Fig. 3.10. All five catheters (volume only) display a strong linear correlation with an $\mathrm{R}^{2}=0.999$, slope of 0.8 and y intercept of approximately $40 \mu \mathrm{S}$. The comparison of the conductance values directly removes possible sources of error such as those in Wei's conversion equation. 


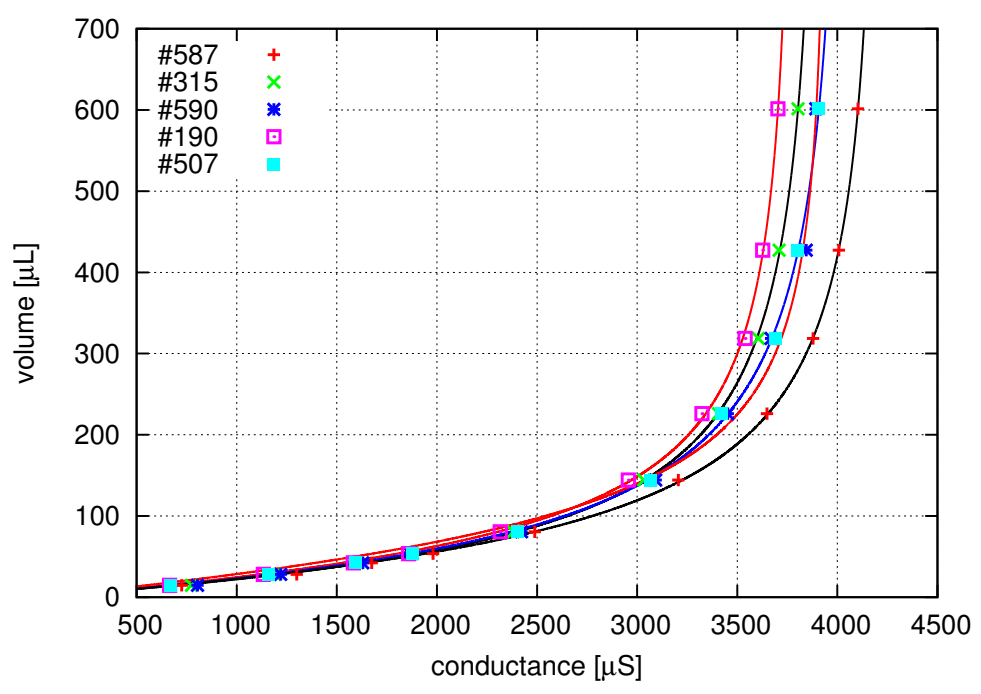

Figure 3.9: Measured conductance vs known cuvette volumes for each catheter. Each data set also includes curve fit.

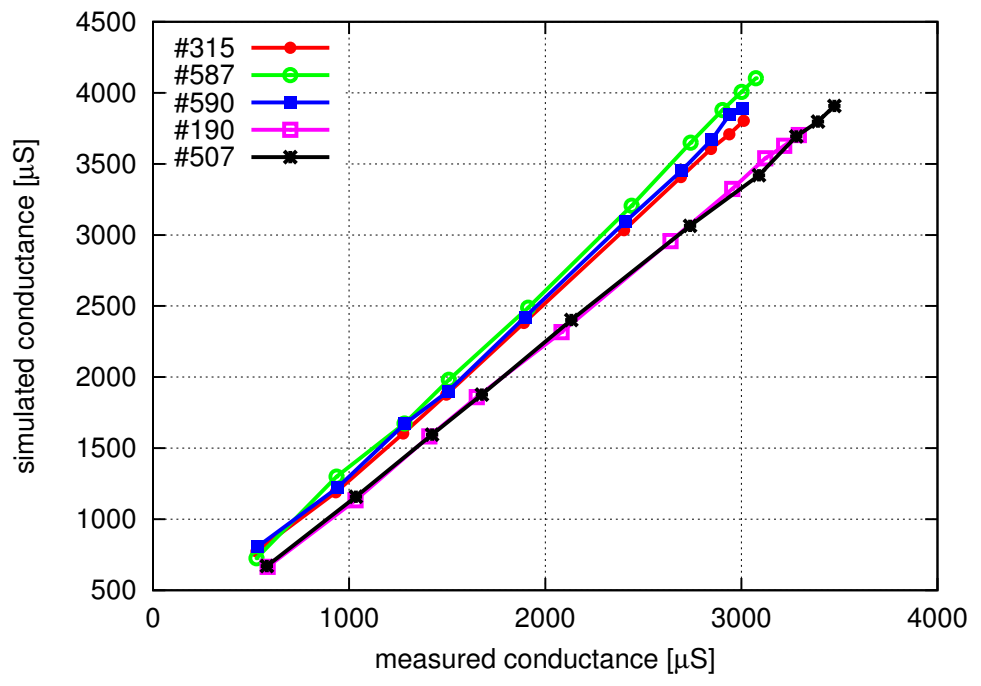

Figure 3.10: Measured vs. simulated conductance for five catheters (volume only) used in the experiment. 


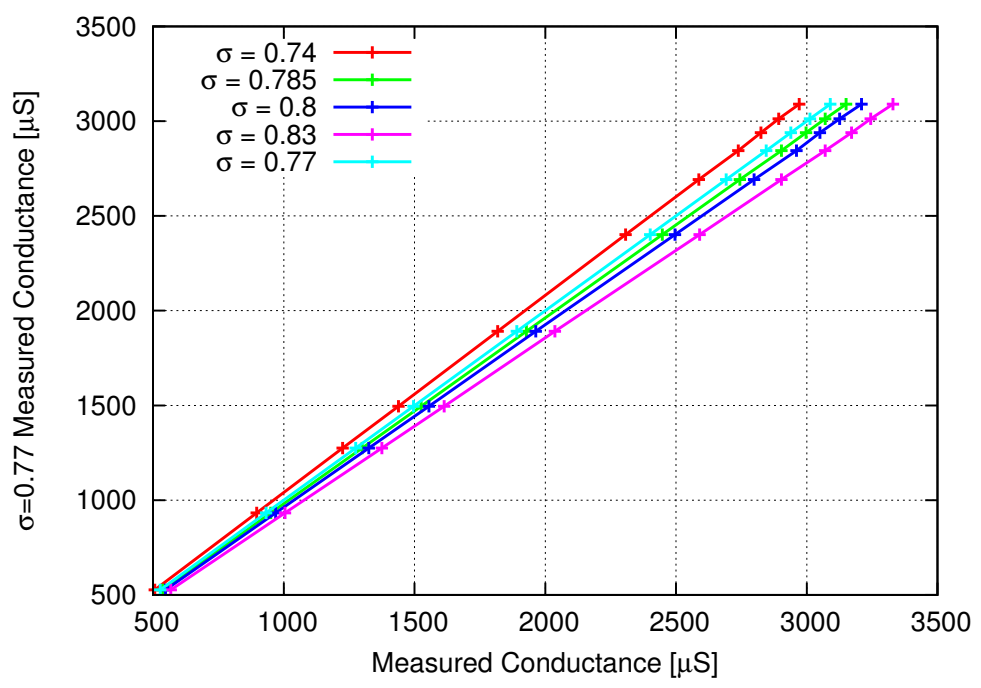

Figure 3.11: Measured Conductance for each different conductivity used vs measured conductance using nominal conductivity value of 0.77 . Conductivity values swept: $0.74,0.785,0.8$, 0.83 , and nominal 0.77 .

The data correlation strongly suggests that the error between simulated conductance and measured conductance is linear and constant between catheters. This means that it can be corrected for with a constant scale and shift factor applied to the simulated data. A possible source of error between the measured conductance and simulated conductance that cannot be accounted for is variance in the conductivity of the saline solution due to evaporation or residue. To evaluate the possible influence of conductivity on the measurements, a simulation is performed with one of the catheter geometries with a sweep of plausible solution conductivity values. The simulations show an average spread of $11.7 \%$ between maximum and minimum conductance values across the volume range, Fig. 3.11. The actual effect of varying conductivity on in-vitro measurements is likely greater in magnitude however it can easily be corrected for once the conductivity of the solution is known. This source of error exists between measured data and simulated data. It does not affect the catheter measurement results because utmost care is taken to ensure the KCL solution does not change conductivity between catheter measurements.

There is however a variation between in-vitro and simulated conductance measurements from each catheter. The magnitude of the variation is much smaller in the simulated results 
Table 3.4: Average Error of Wei's equation vs known volumes for each catheter when using (3.6)

\begin{tabular}{c|c}
\hline Catheter & Avg Error [\%] \\
\hline \hline$\# 190$ & 20 \\
\hline$\# 315$ & 28 \\
\hline $\mathbf{\# 5 0 7}$ & 20 \\
\hline$\# \mathbf{5 8 7}$ & 27 \\
\hline $\mathbf{\# 5 9 0}$ & 30 \\
\hline $\mathbf{\# 8 7 0}$ & 26 \\
\hline $\mathbf{\# 1 1 1 6}$ & 30 \\
\hline \#1147 & 29 \\
\hline Average across 8 Catheters & 26.25 \\
\hline
\end{tabular}

due to there being fewer variables and sources of error. The only difference between the simulations is the measured geometry simulation contains exact measurements, this suggests that the difference is related to catheter geometry. The average error in volume calculated from the measured conductance using Wei's equation for each catheter when compared to the known volume is summarized in Table 3.4 .

Overall, there is on average a $26.25 \%$ error in volume measurements using 8 commercial catheters with nominal $1.2 \mathrm{~F}$ catheter dimensions in 3.6 . The most accurate conductance-tovolume equation (3.6) is used to calculate volumes and the catheters have all undergone the current state of the art in-vitro calibration. The magnitude of this error is much larger than the currently accepted industry standard of $15 \%$. Thus, the source of the error must be explored.

\subsection{Catheter Geometric Sensitivity Analysis}

The average standard deviations $(\sigma)$ in dimensions $(a, b)$ is $22 \mu \mathrm{m}$ and in dimension $L$ is $38 \mu \mathrm{m}$, as measured on the sample of eight catheters (5 volume only, 3 pressure-volume) provided by Transonic Scisense. The largest difference between different catheters in the same dimension is $98 \mu \mathrm{m}$ in $L, 53 \mu \mathrm{m}$ in $a, 63 \mu \mathrm{m}$ in $b$. Thus, the effects of variation in catheter geometry 


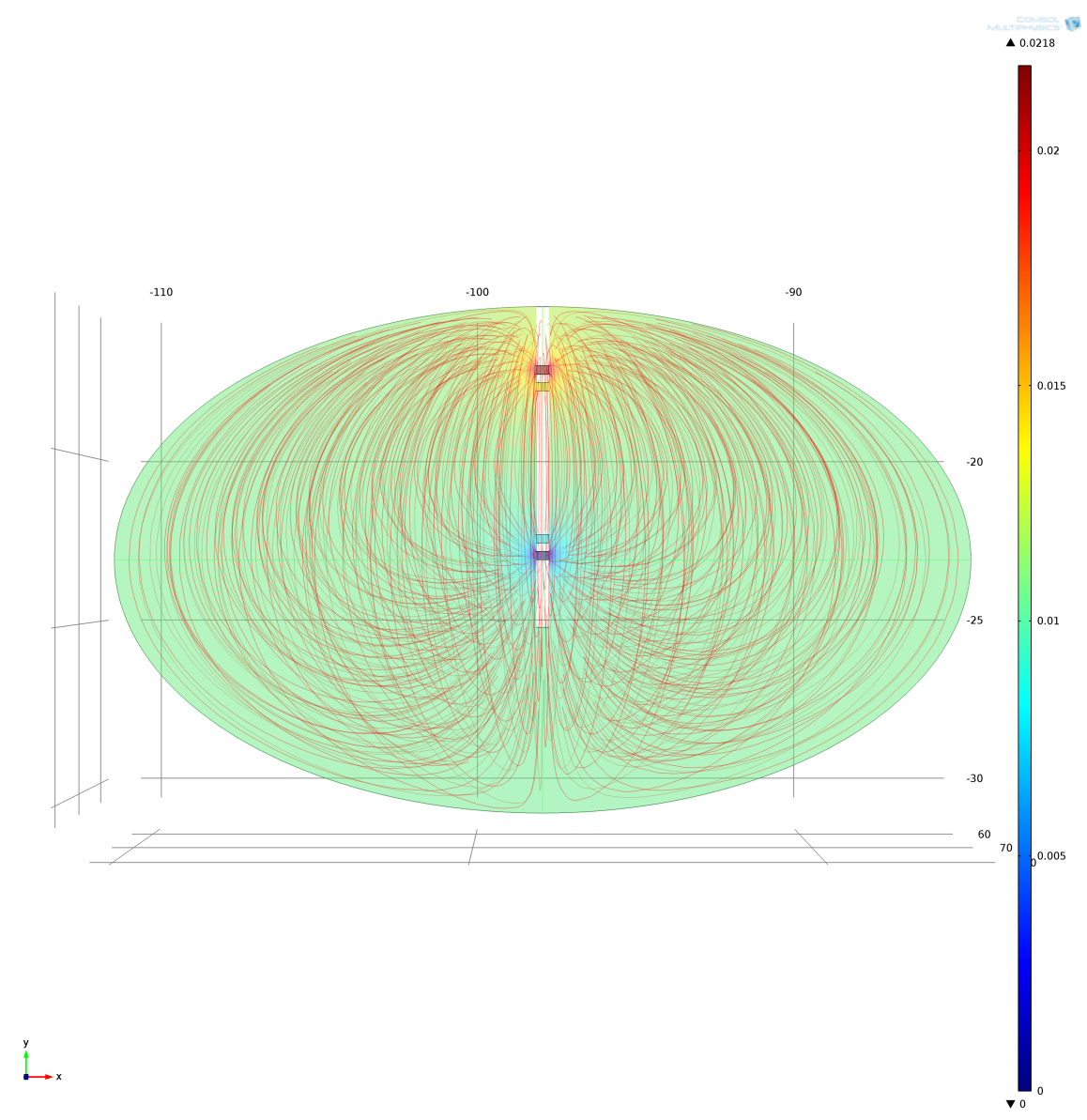

Figure 3.12: COMSOL FEM simulation of a standard geometry catheter in an elliptical cuvette.

$(a, b, L)$ on measured conductance must also be evaluated.

\subsubsection{Catheter Sensitivity Geometric Setup}

In order to simulate the sensitivity in conductance measurements the standard geometry model and both standard and a larger elliptical environment are used, Fig. 3.12, while the catheter geometry is parameterized with dimensions that range $\pm 2 \sigma$ from the volume only catheter dimensions $(190,315,507,587,590)$. Each dimension, $(a, b, L)$ is parameterized individually and the simulation is run for each configuration. All three parameters are then parameterized in the model and each is swept between three values: the ideal dimension value, the ideal dimension value plus $2 \sigma$, and the ideal dimension value minus $2 \sigma$ to create the additional 27 data sets for various volumes $(a=b=0.45 \mathrm{~mm}, 0.5 \mathrm{~mm}, 0.55 \mathrm{~mm}, \mathrm{~L}=4.45 \mathrm{~mm}, 4.5 \mathrm{~mm}$, 
$4.55 \mathrm{~mm}$ ) The effective volume of the cuvette is calculated based on the distance $L$ between the inner electrodes, the radius of the cuvette $R$, and the volume occupied by the catheter body itself.

\subsubsection{Sensitivity Simulation Results}

The simulated conductance values vary for each change in catheter geometry, for both the simulations using cylindrical cuvettes and the simulations using elliptical cuvettes. The variation in calculated volume error using Wei's equation is investigated to determine the possible correlation between catheter geometry and simulated conductance. The simulated conductance measurements from each geometric catheter configuration are converted to volume measurements using Wei's equation (3.6) with ideal geometric parameters. The setup accurately represents the actual application of PV catheters in combination with (3.6) when using the ideal manufacturer dimensions, as only ideal measurements are used in practice. By studying the nature of the variation in volume error produced by Wei's equation 3.6 between data sets, it is possible to determine: a) the degree to which unaccounted variations in catheter geometry effect volume measurements; and b) whether there is a correlation between specific catheter geometry and the resulting volume error.

The plot of root mean square (RMS) volume error generated by sweeping individual catheter dimensions "a" and "L", Fig. 3.13 and 3.14, where root mean square is defined by (3.10).

$$
\text { RMS Error }=\sqrt{(\text { measured value }- \text { known value })^{2}}
$$

The error resulting from sweeping dimension "a" alone and dimension " $b$ " alone are identical due to symmetry and as such only one graph is shown. Both plots show strong linear relationships suggesting that the resulting volume error is significant and correlated to catheter dimensions instead of being random.

The 3D plot of root mean square volume error in Fig. 3.15 is generated by extrapolating 


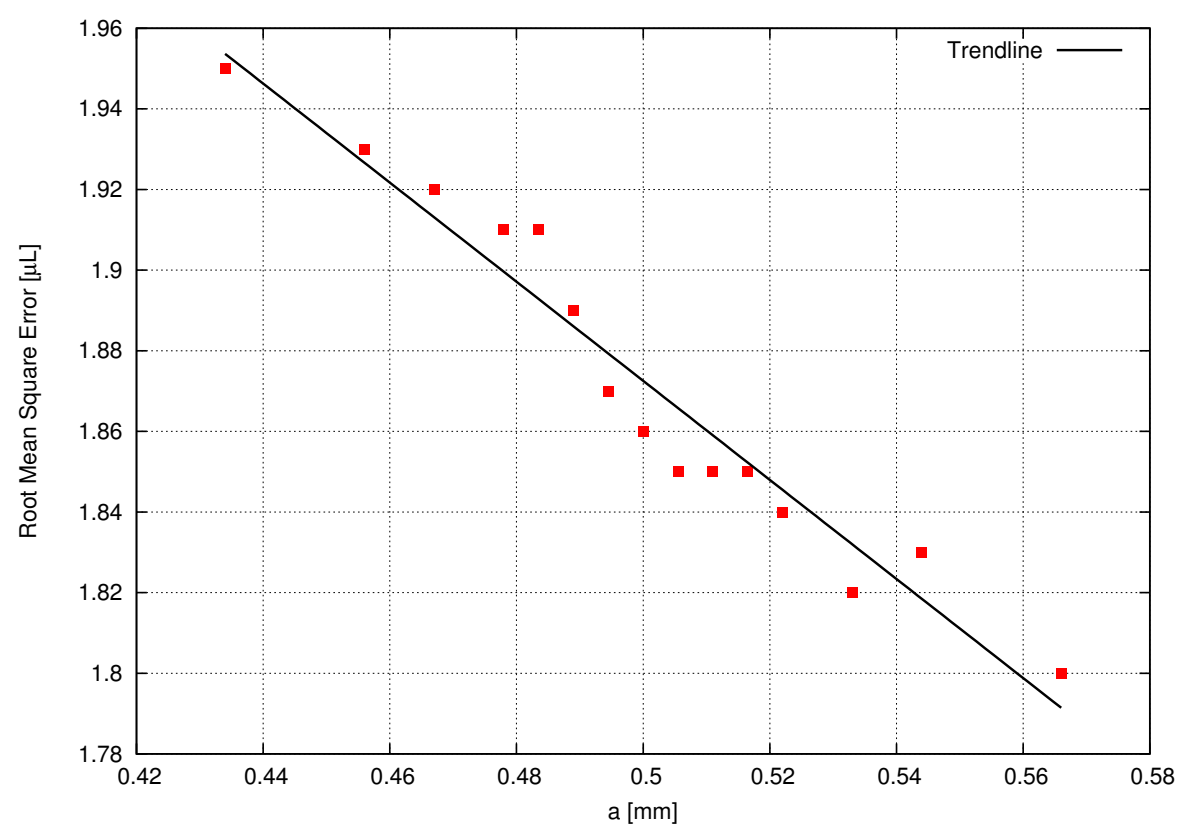

Figure 3.13: Root-mean square error versus catheter dimension a.

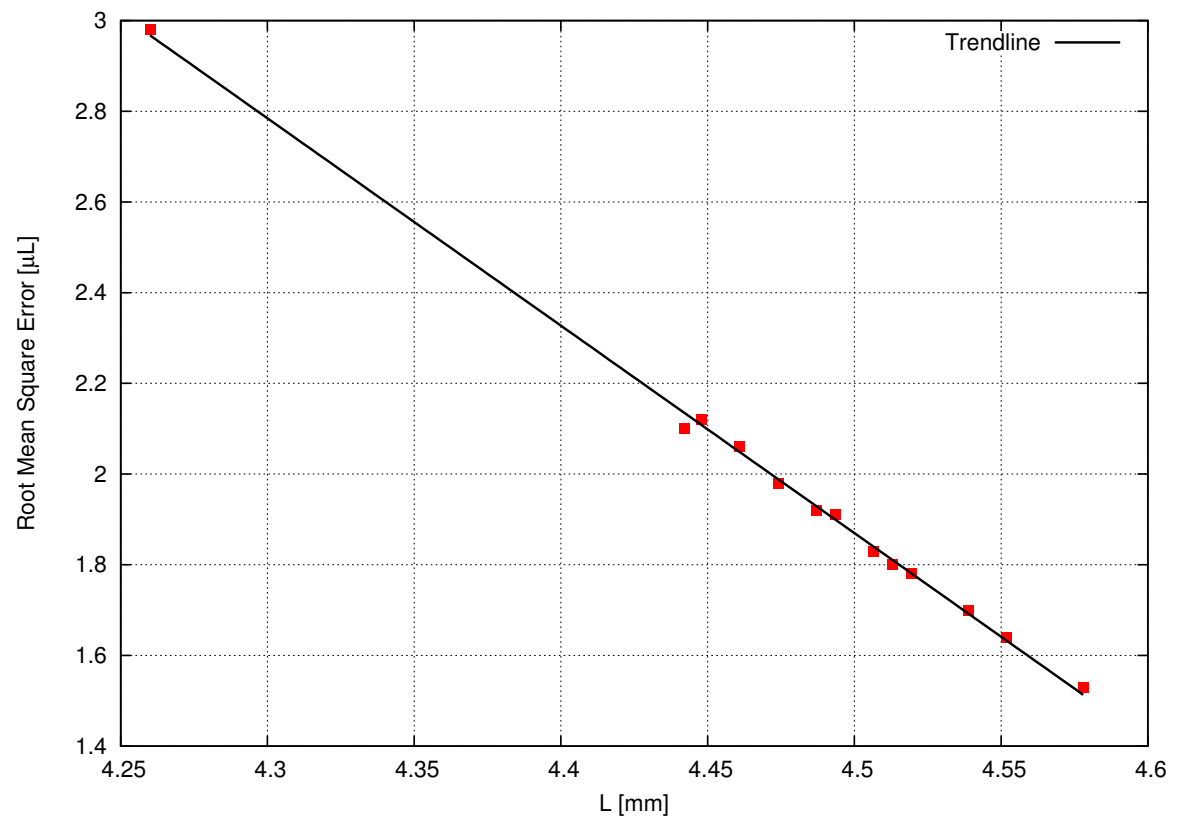

Figure 3.14: Root-mean square error versus catheter dimension $\mathrm{L}$. 


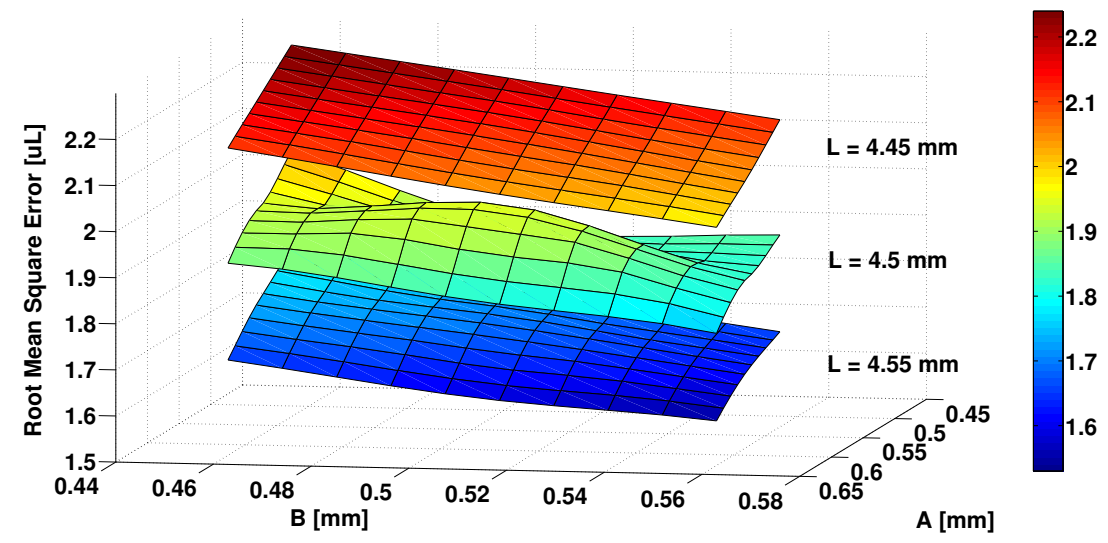

Figure 3.15: Root-mean square error versus catheter dimension a versus catheter dimension $b$ versus catheter dimension L.

the 27 data points generated from the variation of the electrode geometry. The plot shows a predictable and relatively linear relationship between catheter geometry and the resulting volume error. Although the magnitude of the error is relatively small (average of $7 \%$ ) for these simulated data sets, based on the results in Section 3.3.2, it is likely that the magnitude of this error would be larger in an in-vivo experiment.

The volumes that were generated from the a, b, L, values in Fig. 3.15 are also plotted against the correct volumes of the cuvettes. The resulting plots all display a very strong linear correlation with an $\mathrm{R}^{2}$ value greater than 0.99 and a slope of 1 . The only distinction between data sets is the y intercept value that changes in magnitude for each geometric catheter configuration. This value, also known as the calibration factor is always negative, so the absolute magnitude is plotted for of the 27 catheter configurations in Fig. 3.16. A linear fit is generated that has an $\mathrm{R}^{2}$ value greater than 0.9 . This result suggests that there is a linear correlation between catheter geometry and the resulting volume error in volumes calculated by Wei's equation (3.6). Furthermore such a relationship suggests a constant calibration factor can be calculated for each catheter based on solely on the dimensions between the inner electrodes and the dimension between its outer electrode. Since the COMSOL simulations have been proven to correspond in a linear fashion with data measured in-vitro this result suggests that such an equation can be developed for actual catheters. 


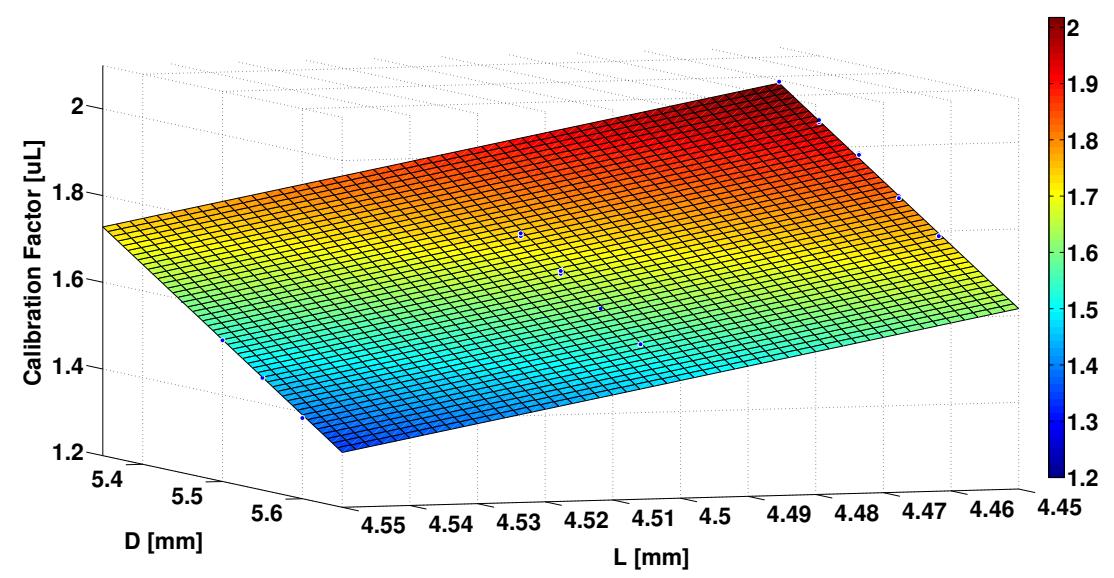

Figure 3.16: Simulated calibration factor versus catheter dimension $\mathrm{d}$ and $\mathrm{L}$ for 27 simulated catheter geometries.

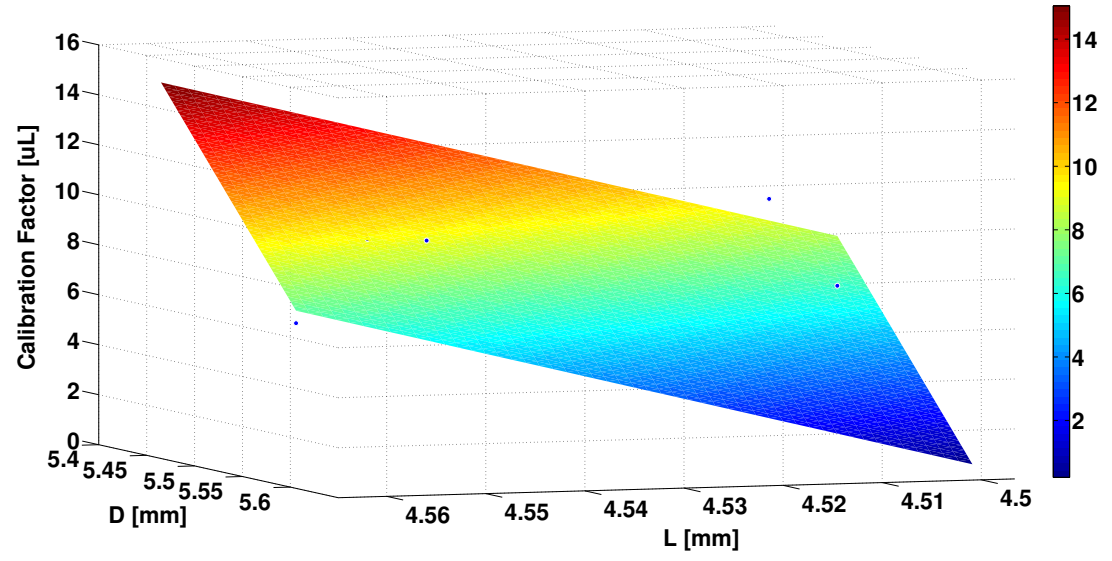

Figure 3.17: Calibration factor versus catheter dimensions $d$ and L, five volume catheters only.

The analysis described previously is applied to the volumes calculated from conductance measured in-vitro. The resulting plot is shown in Fig. 3.17, although it does not display the same linear fit as in Fig. 3.16, this likely due to the fact that 5 sample catheters (volume only) are used. The equation of the plane in Fig. 3.17 is then used as a calibration factor for Wei's equation (3.6).

$$
\operatorname{Vol}(d, L)=\operatorname{Vol}_{\mathrm{W}}-(101 L-56.3 d-138.5)
$$

This equation is then used to calibrate the volume measurements described in Section 3.3 .2 
Table 3.5: Fitting curve coefficients.

\begin{tabular}{rrrr}
\hline Catheter & $x$ & $y$ & $z$ \\
\hline \hline$\# \mathbf{1 9 0}$ & 1071.932 & -0.282 & -1.802 \\
\hline $\mathbf{3 1 5}$ & 463.293 & -0.117 & -1.533 \\
\hline $\mathbf{\# 5 0 7}$ & 446.261 & -0.110 & -1.546 \\
\hline $\mathbf{\# 5 8 7}$ & 819.810 & -0.193 & -1.699 \\
\hline $\mathbf{\# 5 9 0}$ & 4784.047 & -1.209 & -2.301 \\
\hline
\end{tabular}

With the added correction terms, the volume error of Wei's equation after calibration compared to known volumes is reduced to $5 \%$ on average. This is $1 / 3$ of the industry accepted level of error and much lower than the original volume error of $26.25 \%$ Table 3.4 .

Alternatively, the calibrated curves in equation (3.11) may be approximated by fitting curve and the measured data, Fig. 3.9, in the form

$$
\operatorname{Vol}\left(G_{\text {meas }}\right)=G_{\text {meas }} \sqrt{[z] x+y G_{\text {meas }}}
$$

where, the three coefficients are found to be as in Table 3.5 .

\subsection{Conclusion}

In this chapter, a comparison of currently used conductance-to-volume equations was presented, which reveals that Baan's linear equation (3.1) is too simplistic and significantly overestimates volume and as such its use is not recommended. Dubois' equation (3.8) is based on the analytical equation (3.4) and is therefore very inaccurate. It consistently underestimates volumes and its use is also not recommended. Wei's equation 3.6 is the most accurate out of the three and is therefore the only currently viable equation for conductance-to-volume calculations. However, both simulated data and in-vitro measurements suggest that the error in Wei's can be eliminated using a calibration constant. 
Overall, there is an average of $26.25 \%$ error in volume measurements when using Wei's equation (3.6), experimentally,demonstrated, which is much greater than the industry accepted value of $15 \%$. Measured data is used to develop a calibration equation (3.11) that can be used to decrease measurement error to $5 \%$ when using Wei's equation (3.6).

When referring to conductance-to-volume conversion in the rest of this thesis, Wei's equation (3.6) is used. The remaining chapters will discuss the development and testing of the implantable pressure-volume telemetry system. It is important to understand the volume catheter from basic measurement principles (conductance and admittance) to volume conversion to create a fully implantable telemetry system. 


\section{Chapter 4}

\section{Wireless PV Telemetry System*}

A wireless telemetry system is designed that is suited for measuring left ventricle blood pressure and volume data from laboratory animals. The system captures, processes and transmits this data wirelessly to an end base station for further processing. The main design requirements for this system are: it must be small in size and have low power consumption. These two criteria allow a system such as this, to be implanted for a long period of time while operating off of a battery. The overall system is divided into two units: external and implant, Fig. 4.1.

The implantable internal unit is a small application specific system designed to capture biosensor data using a custom sensor front-end, process and communicate the data back to the end device for additional processing. The implantable unit contains four main sub-blocks: power management, processing, RF transceiver, and an custom analog front-end. Each block is discussed in detail in the following sections. The wireless PV telemetry system architecture is comprised of the following four blocks that share a common data and DC power bus: power regulating electronics (PWR), signal processing and control unit $(\mu \mathrm{C}), \mathrm{RF}$ transceiver ( $\mathrm{Rx}-\mathrm{Tx})$, and PV sensor interface (AFE), Fig. 4.1. These modules communicate with each other through a commutation bus. Each module has access to this bus, and is described in more detail in Section 4.3. Physical implementation of the telemetry system is described in Section 4.6 .

*Portions of this chapter are published in NEWCAS 2013 [239], BIOCAS 2013 [31], and published in Springer Biomedical Devices Journal [240|. 


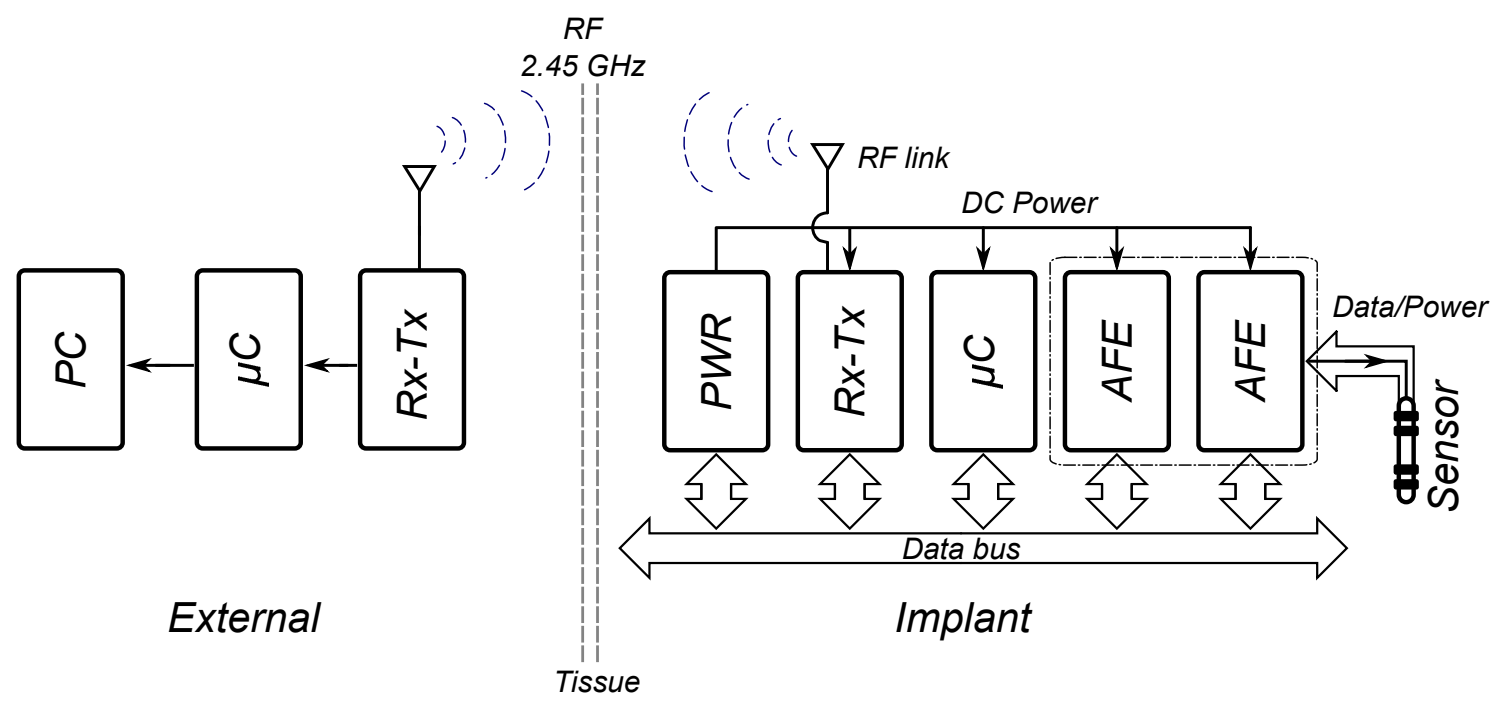

Figure 4.1: Block diagram of the wireless telemetry system environment consisting of the external side (PC, microcontroller, RF transceiver) and the implant (Power, RF transceiver, microcontroller, and analog front end) as well as the pressure-volume sensor.

Additionally, the control architecture and current consumption of the implant are described in Section 4.7 .

The external unit consists of a wireless transceiver, microcontroller, and computer for processing. The computer can be replaced with an application specific system for further reduction in size. Implementation details of the external unit are provided in Section 4.8 .

The following sections describe the design process of the physical implant.

\subsection{Power Module}

This module's purpose is to provide power distribution from the power supply to the rest of the implant. The main components in the power module are: a battery, voltage regulators, and a supervisor device, Fig. 4.2. The power module supplies four voltage rails to the system through the main bus structure: 1) Microcontroller System Voltage (System Voltage - 3.6 V),

2) RF Transceiver Voltage (VCC HF - $3.6 \mathrm{~V}$ ), 3) Interface Voltage (Interface Voltage - 3.6 V), and 4) Virtual Ground (Vtrl Gnd - $1.8 \mathrm{~V}$ ). The system voltage is also referred to as VCC 


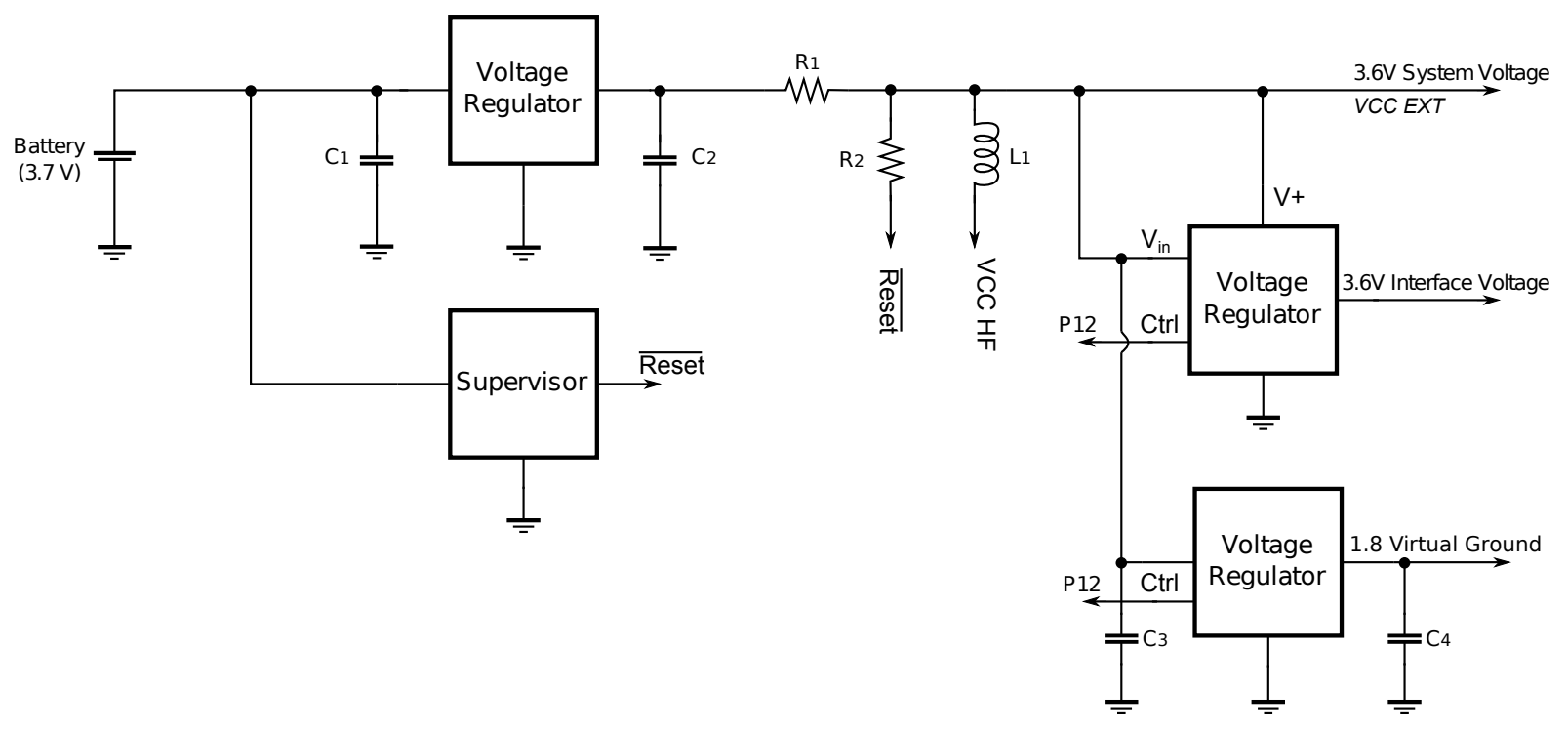

Figure 4.2: Full schematic of the Power Module.

EXT throughout the text, as well the interface voltage is referred to as VCC Int in the text. All modules have access directly to the power module through the communication bus, with each module's ground plane connected directly to the batteries ground.

\subsubsection{Voltage Regulators}

A voltage regulator is used to regulate the input voltage from the battery to some select DC voltage. The regulator should have a low consumption current (Iq), very low dropout (LDO), and have a high power supply rejection ratio (PSRR) to ensure that any transients on the power supply line are not transfered to the systems power supply.

\section{Microcontroller and Transceiver Power Supply}

The power module contains three voltage regulators: 1) to provide power to the microcontroller and transceiver modules, $3.6 \mathrm{~V} \mathrm{2}$ ) to provide power to the interface module, $3.6 \mathrm{~V}$, and 3 ) to provide a virtual ground for the interface module, $1.8 \mathrm{~V}$. The TI TLV70036 is chosen for regulators 1 and 2. This regulator has a fixed $3.6 \mathrm{~V}$ output rather than an adjustable output, with a typical Iq of $31 \mu \mathrm{A}, 175 \mathrm{mV}$ dropout for a $2.35 \mathrm{~V}$ output and a PSRR of $68 \mathrm{~dB}$. In 
addition, the LDO can supply $200 \mathrm{~mA}$ to the system. As a result of having a fixed voltage output, no external resistors are needed to create the regulated output voltage. Although both the microcontroller and RF transceiver operate at $3.6 \mathrm{~V}$, their respective power supply rails (VCC EXT, VCC HF) are separated by a passive network. This network used a single inductor to reduce high frequency noise that may interfere with the transceiver operations.

\section{Interface Power Supply}

The Interface modules operates on the same DC $3.6 \mathrm{~V}$ power supply as the rest of the system, however, as sensor may not be active at all times, this module should be powered down when not in use to reduce the overall power consumption. Furthermore, the microcontroller must be powered at all times to provide proper timing and cannot be actively switched on and off. This prevents the use of a single regulator to power the microcontroller, RF transceiver and interface components. Therefore, a second regulator (3.6 V - TLV70036) is used to provide this voltage rail (Interface Voltage). The enable on the regulator is used to control the power switching. The control line used is P12 (GPIO) from the microcontroller. If P12 is low then the output voltage to the interface modules is off and when P12 is high the output voltage to the interface modules is on. By using this logic, the interface module's power supply can be controlled by sending digital I/O signals from the microcontroller down the bus to the power module to control the operation of the switch.

\section{Virtual Ground}

The need for a voltage reference in the designed wireless telemetry system is to provide a virtual ground to the analog circuitry on the interface modules. A virtual ground is needed to allow for full voltage swing to occur in the analog signal chain while using single ended devices. If full voltage swing is needed in any module, a virtual ground of VCC EXT/2 = 3.6/2 =1.8 V is required. Initially, a series voltage reference was chosen, however, after experimentation it was determined that additional current sourcing was required for the actual circuit. Therefore, 
a low-power LDO, Analog Devices ADP160, is used instead.

The ADP160 has an output voltage of $1.8 \mathrm{~V}$ with a min voltage of $1.7964 \mathrm{~V}$ to a maximum output of $1.8036 \mathrm{~V}$ and can supply a maximum of $150 \mathrm{~mA}$ of current. This LDO is also chosen for its low supply current of $860 \mathrm{nA}$ with a wide input range. In addition, the ADP160's enable is controlled via the same signal (P12) as the interface voltage regulator, thus both regulators are switched on and off at the same time.

\subsubsection{Supervisor Device}

A supervisor integrated circuit (IC) is used in the power module to provide circuit initialization and voltage protection to the microcontroller and battery. The supervisor circuit is connected to the microcontroller's reset pin where an internal delay is started to prevent the microcontroller from powering until the line voltage is stable and above a specific voltage. If the line voltage or in our system, the output of the battery, drops below the specific voltage the supervisor chip will cause the microcontroller to reset and prevent damage to the microcontroller. The supervisor chip used is the TI TPS3838 with a typical power consumption of $220 \mathrm{nA}$ and a supervisor (threshold) voltage of $2.93 \mathrm{~V}$.

\subsubsection{Battery}

A battery for an implantable system should be small in size but have a large capacity. It also should be rechargeable in order to extend the lifetime of the system. Lithium-ion polymer batteries are chosen for the batteries for this system because of their high capacity and small size characteristics, as well as being rechargeable.

The Full River $501213 \mathrm{Li}-$ Poly battery is chosen for this project and has a $40 \mathrm{mAh}$ capacity with a nominal voltage of $3.7 \mathrm{~V}$ and a charge condition of $1 \mathrm{C}$. The overall dimensions are 15 $\mathrm{mm} \times 11.0 \mathrm{~mm} \times 5 \mathrm{~mm}$, Fig. 4.3. The overall area is the most important design constraint for the battery as this directly dictates the system size. The batteries are recharged with a Li-Poly USB battery charger. 


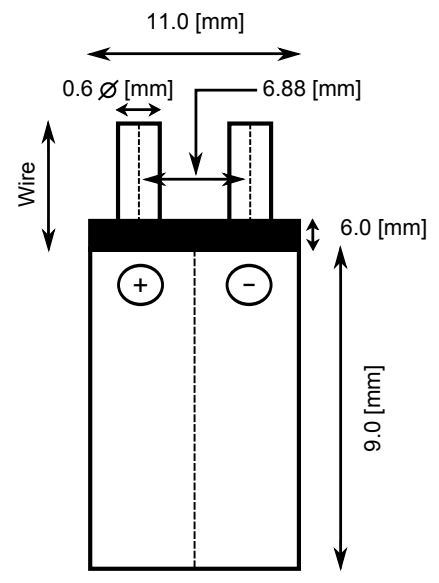

a)

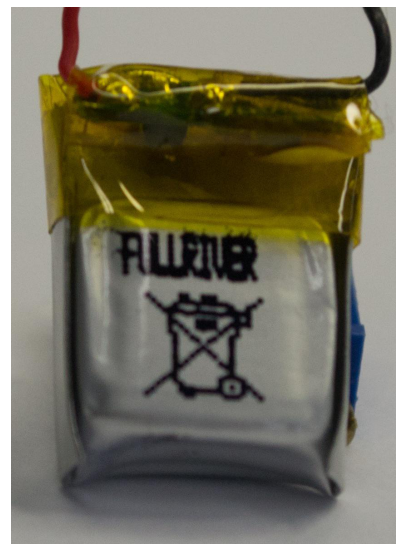

b)

Figure 4.3: a) Dimensions of the battery used in the implantable system; b) Physical battery.

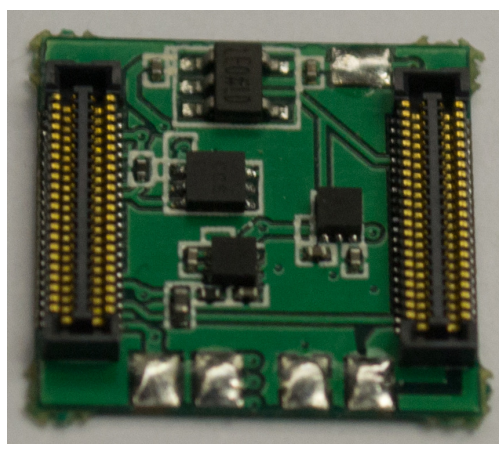

Figure 4.4: Power Module Physical Implementation.

\subsubsection{Power Module Implementation}

The ICs and passives are located on the top side of the PCB, leaving the battery to be located on the bottom, Fig. 4.4. The $40 \mathrm{mAh}$ battery is designed to fit the desired board dimensions and is soldered on to the bottom of the PCB using two wires and glue for stability purposes. The supervisor IC and the $3.6 \mathrm{~V}$ regulators are both no lead packages to reduce the physical footprint and the $1.8 \mathrm{~V}$ regulator is contained in a SOT-23 package. The battery is charged externally via a $5 \mathrm{~V}$ connection to the communication bus, accessed by the development module.

Additional test pads are added to the power module to provide testing and fail safes to the system. Three test pads are added to test the main power supply voltage, ground and the $1.8 \mathrm{~V}$ voltage reference. These pads are added to test the power module directly without the external 
development board. Two additional pads provide direct access to the battery for charging purposes. These pads are added to provide charging access to the battery if the vertical bus happens to fail. It should be noted that an external charging PCB should be used in all battery charging cases to ensure safe charging. Additional information on battery charger is provided in Section 4.5.2. If unregulated voltage is directly applied to these pads, the battery may be damaged due to incorrect voltage and current charging characteristics.

\subsection{Processing and RF Modules}

Data capture and processing is completed by a low-power microcontroller, Texas Instruments (TI) MSP430, located in the microcontroller module. The analog data from the PV sensor interface is digitized by the internal 10-bit A/D converter and the digital PV datastream is sent via the Serial Peripheral Interface (SPI) bus to the RF transceiver.

RF transmission from the implant to the external unit is completed by the low-power TI CC2500 radio transceiver. The RF data is transmitted at $2.45 \mathrm{GHz}$ using Frequency Shift Keying (FSK) modulation, thus, allowing the system to work in the Industrial, Scientific and Medical (ISM) band. Each module along with its sub-modules will be discussed in detail below.

\subsubsection{Microcontroller Module}

The Microcontroller module is the main communications hub between the RF electronics, interface electronics and power electronics, Fig. 4.5. The main duties of this module are to capture the incoming analog data from the interface module, digitize it and then process the data for RF transfer. The transfer of digital data to the RF radio is transferred via the SPI bus, discussed in more detail in Section 4.2.2. The analog data is transferred down to the microcontroller module through the communication bus and connected directly to the microcontroller's input pins. This data is then digitized by a 10-bit ADC, processed and sent to the CC2500 


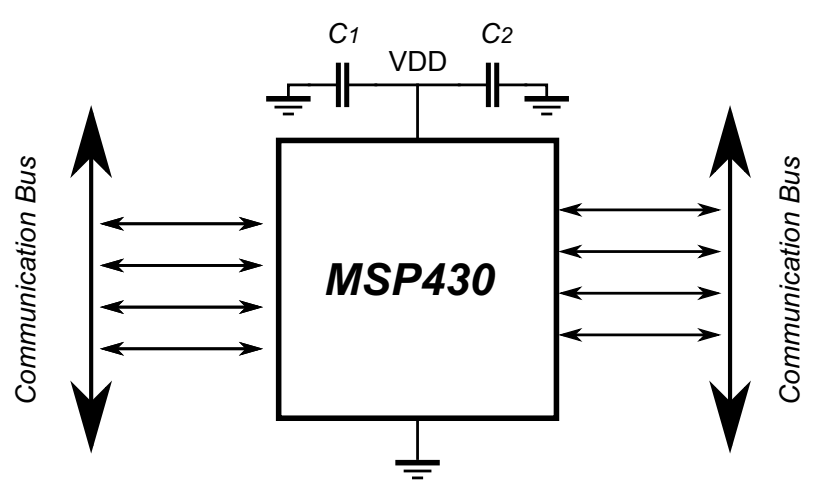

Figure 4.5: Microcontroller Module schematic. Input and output communication through communication bus.

for RF transfer. The microcontroller module is programmed through a external programming connector that is disconnected after each upload.

\section{MSP430}

Various microcontrollers exist that perform basic functions such as general digital input and output (GPIO), analog input and communication protocols. These microcontrollers come from a wide range of manufactures such as Atmel, Texas Instruments, PIC, etc. The microcontroller chosen for this implant is the MSP430 from Texas Instruments. It is chosen for its wide availability, low cost, and low power consumption. The MSP430 comes in may different versions, each with different features, the MSP430F2274 is chosen for this project. The MSP430F2274 contains a 12 channel 10-bit SAR ADC, SPI communication bus, a $16 \mathrm{MHz}$ internal clock and many more features within a 40 pin package [241]. All pins of the microcontroller are connected to the main communication bus, allowing each module to communicate directly with the microcontroller.

\section{MSP430 Timing}

The MSP430F2274 contains a clock module which includes three main clock sources, LFXT1CK, DCOCLK, VLOCLK. Each clock source can be used to drive any of the three main clock signals: ACLK, MCLK, SMCLK. The low-frequency oscillator is the clock source for external 
crystals and resonators, with a frequency range of $400 \mathrm{kHz}$ to $16 \mathrm{MHz}$. The internal Digitally Controlled Oscillator (DCO) is the main clock inside the MSP430, with a maximum frequency of $16 \mathrm{MHz}$ and is able to be divided internally to create sub frequencies. Finally the VLOCLK is an internal low-power, low-frequency oscillator in the frequency range of $12 \mathrm{kHz}$. Each of these clock sources are used to route the clock signals to the MSP430's internal architecture. The main clock signal of the MSP430 is MCLK, which is used by the CPU and the supporting architecture. The remaining clock signals, ACLK (Auxiliary Clock), SMCLK (Sub-Main Clock), are used to control individual peripheral modules.

In the designed telemetry system, the MSP430F2274 is clocked using the internal clock rather than an external crystal. An external crystal is not used to reduce area and component count of the system. The MCLK is sourced from the DCO using a internal frequency of $8 \mathrm{MHz}$ and ACLK, SMCLK are sourced by the VLOCLK. A lower clock frequency is used in the DCO instead of the higher maximum frequency of $16 \mathrm{MHz}$ to conserve power during active modes of the MSP430. Additional information is provided in Section 4.7 on how each clock is utilized within the control architecture.

\section{Analog to Digital Conversion in the MSP430}

The analog-to-digital (ADC) conversion module is an essential module for the operation of the wireless telemetry system. The ADC is used to convert the analog sensor data to usable digital data. The MSP430 contains a 10-bit successive approximation ADC with eight external inputs. The voltage reference that is used for comparison can be an external or internal voltage reference. Any clock in the MSP430 can be used to control the conversion timing.

The main formula used in the conversion of the analog signal to the 10-bit digital signal is (4.1) [242], where $\mathrm{V}_{\text {in }}$ represents the analog input voltage, $\mathrm{V}_{R+}$ indicates the positive voltage reference used, and $\mathrm{V}_{R_{-}}$indicates the negative voltage reference used, in most cases $0 \mathrm{~V}$.

$$
N_{A D C}=1023 * \frac{V_{\text {in }}-V_{R-}}{V_{R+}-V_{R-}}
$$


Table 4.1: TI MSP430 Low Power Modes and Clock Status

\begin{tabular}{l|l}
\hline Mode & CPU and Clock Status \\
\hline Active & CPU is active, all enabled clocks are active \\
\hline LPM 0 & CPU, MCLK are disabled, SMCLK, ACLK are active \\
\hline LPM 1 & $\begin{array}{l}\text { CPU, CLK are disabled, DCO and DC generator are disabled if the DCO } \\
\text { is not used for SMCLK. ACLK is active }\end{array}$ \\
\hline LPM 2 & $\begin{array}{l}\text { CPU, MCLK, SMCLK, DCO are disabled DC generator remains enabled } \\
\text { ACLK is active }\end{array}$ \\
\hline LPM 3 & $\begin{array}{l}\text { CPU, MCLK, SMCLK, DCO are disabled DC generator } \\
\text { disabled ACLK is active }\end{array}$ \\
\hline LPM 4 & CPU and all clocks disabled \\
\hline
\end{tabular}

In this system, three analog inputs are used, with each using $\mathrm{VCC}(3.6 \mathrm{~V})$ as the positive voltage reference rather than the external voltage references as less external components are needed, allowing the reduction of the area of the overall system. The internal ADC oscillator is used to clock the conversion of the analog to digital signal. The sampling frequency of the ADC is determined by the control architecture described in detail in Section 4.7.

\section{Power Management}

One of the key features of the MSP430 is that its designed for low-power applications, which makes it an ideal choice for the implantable system. The MSP430 has six different operating modes designed specifically for low power operation, Active, LPM $0-4$. These modes range from $270 \mu \mathrm{A}$ at $1 \mathrm{MHz}, 2.2 \mathrm{~V}$ supply to $0.7 \mu \mathrm{A}$ in LPM4.

The main difference between each mode is the number of clocks in the clocking module that are active. When in the active mode, all clocks and the CPU are active, while at the other extreme in LPM4, all clocks and the CPU are disabled. Low Power Mode (LPM) 0 to LPM 3 have various clocks on or off, Table 4.1. Ideally in an implantable system, the MSP430 would remain in LPM4 for most of the time, only to be placed in one of the other low power modes when processing is needed, this is done using an interrupt. By using these different operating modes, the power consumption of the wireless system can be greatly reduced. 


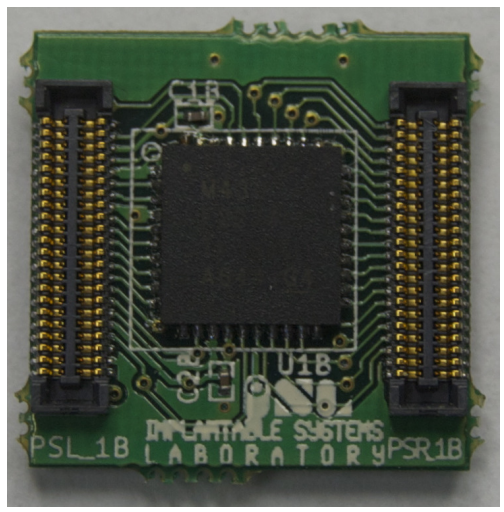

Figure 4.6: Microcontroller Module Physical Implementation.

\section{Firmware}

The MSP430 is programmed using IAR Embedded Workbench for MSP430 IDE. MSP430 assembly and $\mathrm{C}$ are the main programming languages used in this implantable telemetry system. For the preliminary tests of the MSP430, assembly language is used to test each register and specific functions while $\mathrm{C}$ language is used for higher level programming.

The MPS430 is programmed using a 6 pin custom connector which contains the pins: Power, Ground, TX, RX, SBWTCLK and SBWTDIO. The power and ground pins are connected to the USB power and ground to supply power to the microcontroller while programming. The TX and RX pins are the UART data lines used in communication between a computer and the microcontroller. Finally, the SBWTCLK and SBWTDIO pins and used by IAR Embedded Workbench for programming and resetting the microcontroller. The main telemetry system control firmware along with power consumption details are discussed in Section 4.7 .

\section{Microcontroller Module Implementation}

The microcontroller is physically constructed by a single 40 pin no-lead package along with headers and receptacles for insertion into the communication stack, Fig. 4.6. 


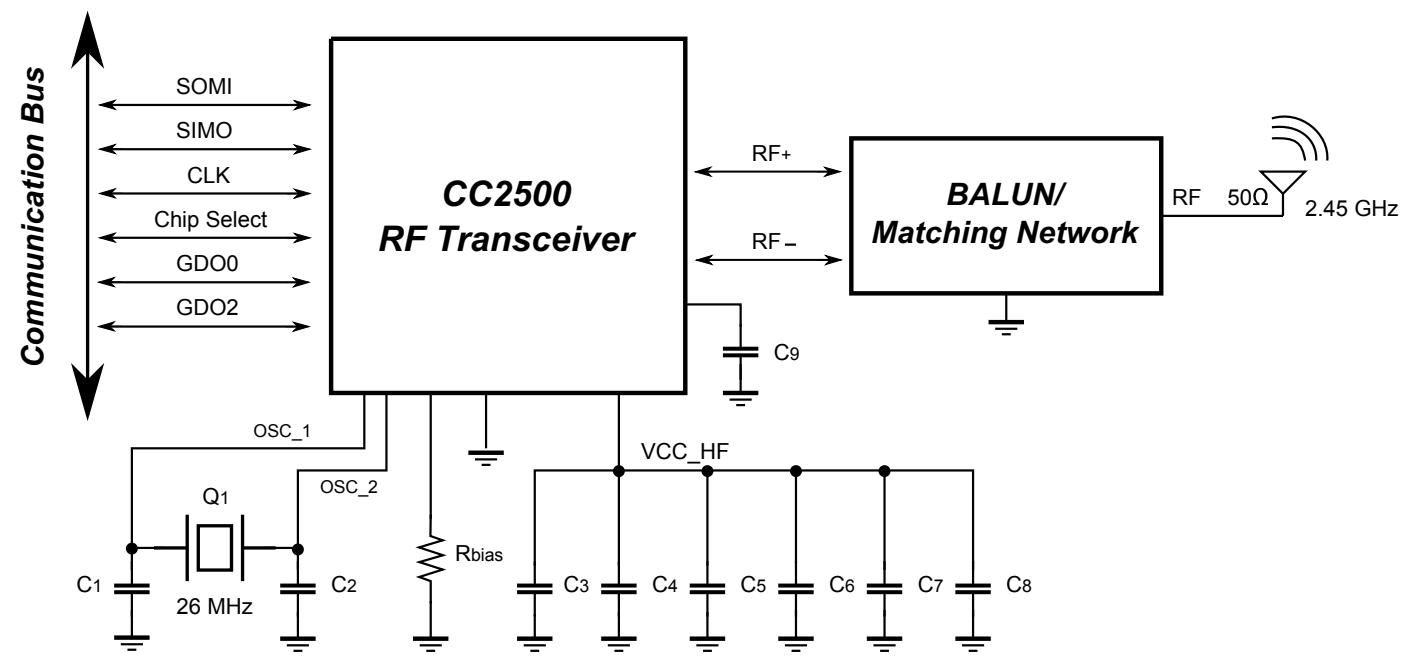

Figure 4.7: Radio Module schematic with SPI communication through the bus. Matching network and RF antenna are also included.

\subsubsection{Radio Module}

The radio module inside the telemetry system enables wireless communication to an end device and contains four major components: RF transceiver, matching network, antenna, and a clock source, Fig. 4.7 .

This module directly interfaces with the Microcontroller Module through the communication bus, with no other modules interfacing with it. The radio module transmits its data wirelessly via a RF antenna and FSK modulation. Different wireless protocols exist to transmit data wireless such as bluetooth, WIFI, non-standard $2.4 \mathrm{GHz}$, other RF proprietary RF frequencies, etc. A non-standard TI $2.4 \mathrm{GHz}$ protocol is chosen for its low-power features and its high compatibility with other Texas Instruments components.

The RF transceiver must interface with an external antenna that will allow it to transmit to a base station. Generally, this must be done through a matching network, matching the output impedance of the transceiver to the $50 \Omega$ antenna, discussed below.

Along with a matching network and antenna, the RF transceiver must also contain a clock to correctly time the communication between the RF transceiver and the microcontroller, a 26 MHz quartz crystal is used to achieve this function. 


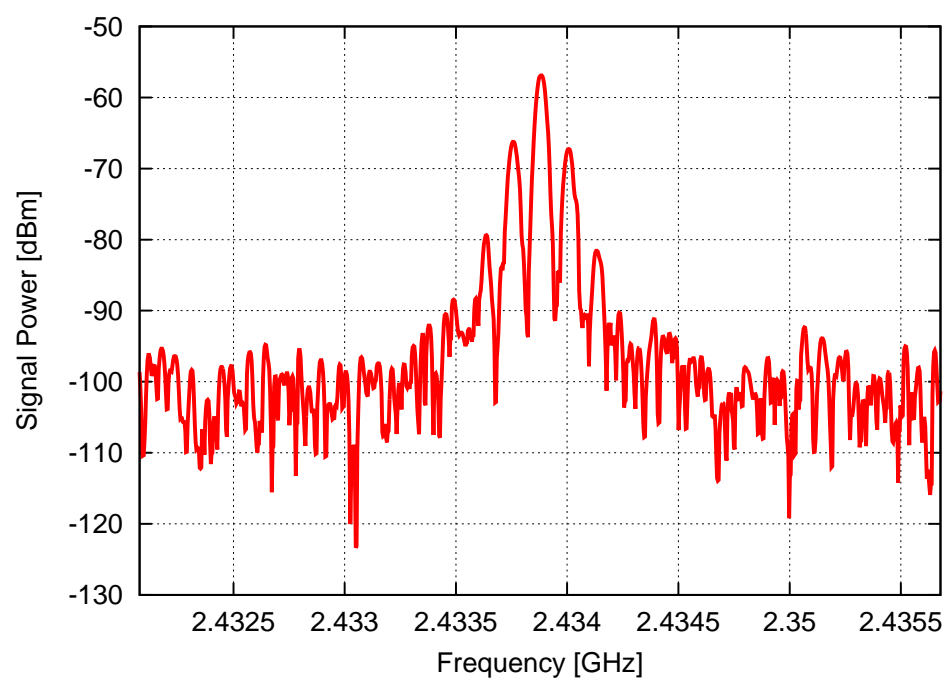

Figure 4.8: Frequency bandwidth of RF transceiver at a distance of $12 \mathrm{~cm}$, Tx output power $=$ $-12 \mathrm{dBm} \mathrm{F}_{\text {Centre }}=2.433592 \mathrm{GHz}$.

\section{$\mathrm{CC2500}$}

The CC2500 is a low-power $2.4 \mathrm{GHz}$ wireless transceiver and is designed for the Industrial, Scientific and Medical (ISM) band along with the Short Range Device (SRD) band, $2400 \mathrm{MHz}$ $-2483.5 \mathrm{MHz}$. The CC2500 has a programmable output power up to $+1 \mathrm{dBm}$, with a maximum data rate of $500 \mathrm{kBaud}$. The CC2500 acts as a receiver as well, with a sensitivity of $-104 \mathrm{dBm}$. Typically, when the implant is in normal operation, the transceiver output power is set to -12 $d b m$ within the frequency bandwidth of (2.4320-2.4356) GHz, Fig. 4.8

The CC2500 is chosen for the RF module for its low current consumption (400nA in sleep) and minimal external components required. The CC2500 is programmed by the MSP430 directly through the SPI bus discussed below. The CC2500 is clocked using an external $26 \mathrm{MHz}$ crystal, with two $27 \mathrm{pF}$ capacitors connected directly to the IC. Additional external components are needed for decoupling capacitors, bias resistors and matching network circuitry.

The CC2500 interfaces with a $50 \Omega$ antenna through a balun and matching network. The RF input and output of the CC2500 is balanced with an impedance of $Z_{\text {out }}=80+j 74 \Omega$. A balance to unbalanced (balun) network is generally created from passive components to convert 


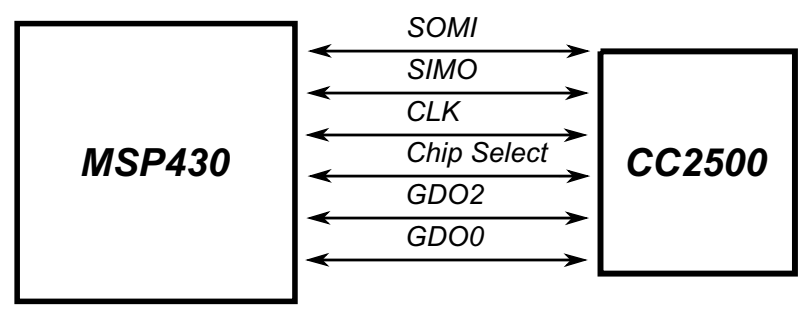

Figure 4.9: SPI digital communication link between MSP430 and CC2500.

the balanced signal, i.e., differential in/out to unbalanced, i.e., single ended $50 \Omega$ antenna. This intermediate impedance is then matched to the $50 \Omega$ antenna for maximum power transfer. The physical implementation of the balun and matching network are described in Section 4.2.2.

\section{Communication Between Microcontroller and CC2500}

The digital communication between the microcontroller (MSP430) and the transceiver (CC2500) is achieved through the SPI bus. Six wired connections make up this bus, four for the main SPI link and two for interrupts, Fig. 4.9. The four SPI wired connections, SPI SIMO, SPI SOMI, SPI CLK, and SPI Chip Select send and receive data from the CC2500. SPI Chip select is used to enable and disable the communications between the two devices. This pin is essential in the design if additional connections are made to the SPI bus. In this specific case, only a single SPI device is used. The timing for the SPI communication is preformed by the SPI CLK pin. This timing is synchronized between both the master and salve devices using this pin automatically. Finally, the two main data lines, SPI SIMO, SPI SOMI, represent the master to slave communication and the slave to master communication, respectively. In this case, the MSP430 acts as the master and the CC2500 as the salve device. The CC2500 will send back status packets along with any data that is received.

The other two connections, are used for interrupts to allow the CC2500 to trigger the microcontroller to wake up from low-power state when a packet is received. The CC2500 refers to these two pins as GDO0 and GDO2. In the designed telemetry system they are connected to GPIO pins 2 and 3 on the MSP430. By using these two interrupts, the MSP430 is able to go in 
low-power state while the CC2500 remains in receive mode.

The SPI bus is directly integrated in the main communication bus structure. By doing this, any module can access the SPI bus to communicate digitally with the microcontroller module, only an additional chip select pin is needed to determine which device to communicate with.

\section{RF Parameters}

The CC2500 includes 47 programmable registers that contain the parameters used in RF transmission. Examples of these registers include configuration for modulation type, frequency, output power, packet size, etc. These registers are all programmed through the SPI bus from the MSP430. Upon power up, these parameters are copied to the registers from the MSP430, as the CC2500 does not store them after loss of power. The CC2500 is configured to use Frequency-Shift-Keying (FSK) Modulation and an operating frequency of $2.45 \mathrm{GHz}$. These parameters can be determined via a TI tool, SmartRF, or manually from the CC2500 datasheet.

\section{RF Module Implementation}

The RF module contains the RF transceiver, balun, crystal, antenna, and various required passive components, Fig. 4.10. The RF transceiver, CC2500, is a no-lead package that requires six decoupling capacitors and a bias resistor. The $26 \mathrm{MHz}$ crystal is packaged in a $2 \mathrm{~mm}$ x 1.6 $\mathrm{mm}\left(3.2 \mathrm{~mm}^{2}\right)$. When miniaturizing the system, traditional passive component balun/matching networks are too large to fit in the required small PCB footprint. Thus, a commercial ceramic balun/matching network is used, Anaren BD2425N50ATI [243].

The Anaren BD2425N50ATI is a single $1.05 \mathrm{~mm}$ x $1.05 \mathrm{~mm}$ x $0.65 \mathrm{~mm}$ mutli-layer ceramic structure and 2 external passive components. The Anaren balun along with 2 external components, $10 \mathrm{nH}$ inductor and $5.6 \mathrm{pF}$ capacitor, is designed specifically for the CC2500 RF transceiver used in the RF module's architecture. It is made to convert the balanced output impedance of the CC2500 to an intermediate unbalanced impedance and then convert it to 50 $\Omega$ to be connected to a RF antenna. 

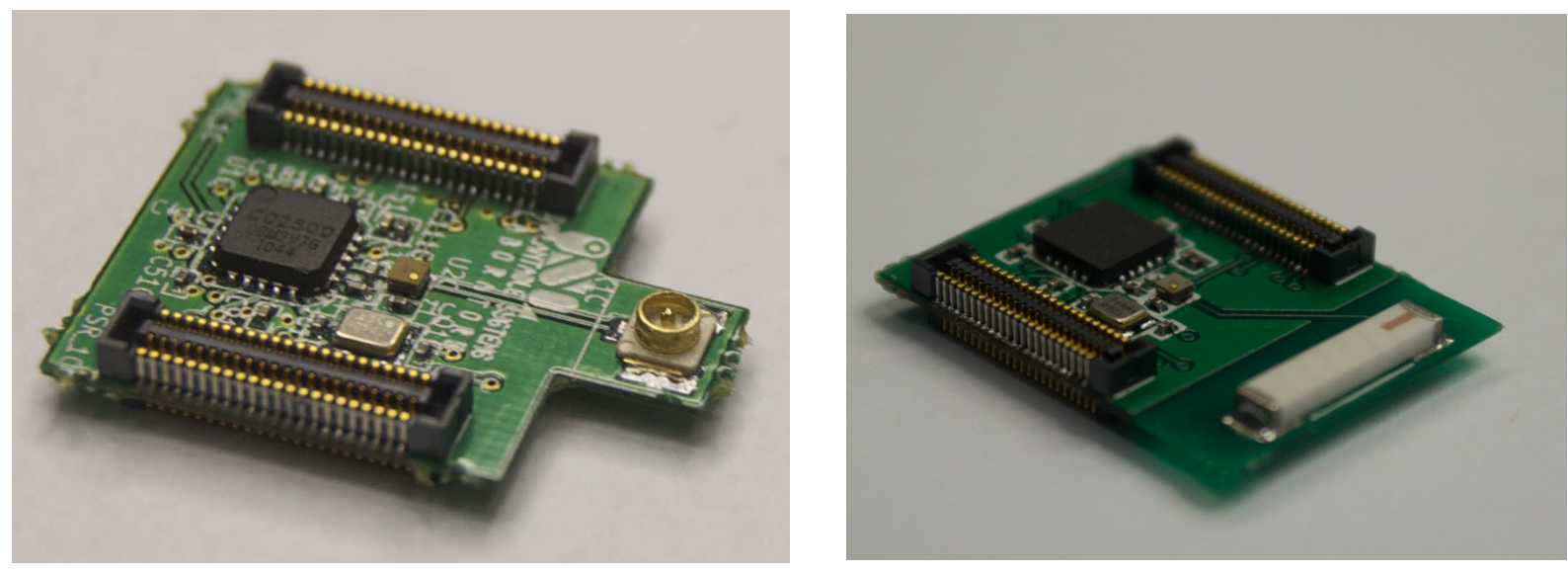

Figure 4.10: Radio Module: Left with external antenna, Right with PCB antenna.

Two versions of the RF module with different antenna configurations are created for testing: a) with an external connector, Fig 4.10 left, and b) with an internal PCB antenna, Fig.4.10 right. First, the use of an miniture RF connector, TE Ultraminiature Coax Connector (UMCC) [244], allows for the testing of different types of antennas as well as it keeps the internal size of the implant small. The UMCC connector is $3 \mathrm{~mm}$ x $3 \mathrm{~mm}$. The UMCC can interface with various larger connectors such as SMA, BNC, etc using $50 \Omega$ adapter cables. For this design, a 30 mm 2.4 GHz antenna is attached to the UMCC for use. A detailed phantom tissue test for this antenna along with transmitted and recevied power details is outlined in Section 5.1 .

The second version uses a traditional PCB antenna $(2 \times 11) \mathrm{mm}$ that is soldered directly onto the PCB board. The PCB antenna does not allow for the freedom of different antenna locations or sizes. However, its benefit is that it is robust and minimizes possible failure points (UMCC connector or external antenna itself). Additional details are provided in Section 5.1 along with which version was used in the implant study.

The entire RF module is fits on a $15 \mathrm{~mm}$ x $15 \mathrm{~mm}$ PCB however, an extension is made to allow the mated height ( $2 \mathrm{~mm}$ ) of the RF connector or the height of the PCB antenna to not interfere with the internal clearance of the stack. 


\subsection{Communications Bus}

The telemetry system's main form of digital and analog communication between each module and the outside world is achieved through the use of a communications bus. When designing the bus structure multiple signals are required and must be taken into consideration. These include: power signals (System Voltage, Interface Voltage, HF voltage), ground signals, I/O, programming pins, charging pins, etc. With the physical design in mind, the communication bus is split across two vertical connectors. The designed implant contains a communication bus with a total of 88 pins, Table 4.2. In this bus, the 88 connections can be broken into the following groups: power signals, ground, SPI, programming, and general input and output.

The system voltage and HF voltage pins have two pin allocations each. The interface voltage rail provides power to the interface module through a the $3.6 \mathrm{~V}$ and $1.8 \mathrm{~V}$ regulators located on the Power Module, Section 4.1.1. A $5 \mathrm{~V}$ power rail is required for USB charging directly to the battery. There are a total of 44 ground connections used in this architecture of the Communications Bus. The additional grounds do not affect the overall function of the system but do provide additional connections on each module, simplifying the layout. Furthermore, programming and SPI pins are also located on the bus.

Twenty seven I/O pins are connected to the bus. Here, the pins are allocated into six specific categories: general I/O, enable pins, analog inputs, Interface Module outputs, and Interface Module separation. There are 16 digital general I/O ports and four analog inputs that are unallocated on the bus, for future use if needed. A single enable pin is used to control the Interface Module's power supply.

Three signals are used in the PCB seperation of the Interface Module, Gain Stage Out, Q Gate, and I Gate. The details of these signals are described in Section 4.4. Pins P43, P44, and P45 are used as the main outputs of this module where P43 represents the Q Out, P44 the I Out and $\mathrm{P} 45$ represents the output voltage related to pressure output. 
Table 4.2: Communication Bus in the second prototype

\begin{tabular}{ll}
\hline Pin & Function \\
\hline System Voltage & 3.6 Volt Rail for System Voltage - From Power Module \\
\hline HF Voltage & 3.6 Volt Rail for RF Module - From Power Module \\
\hline Interface Voltage & 3.6 Volt Rail for Interface Module - From Power Module \\
\hline 1.8 V Reference & 1.8 Volt Reference for Interface Module - From Power Module \\
\hline Ground & Ground - Connected Directly to the Battery \\
\hline UCB0STE & Chip Select for SPI Bus \\
\hline UCB0SIMO & Slave In, Master Out for SPI Bus \\
\hline UCB0SOMI & Slave Out, Master In for SPI Bus \\
\hline UCB0CLK & SPI Clock \\
\hline GDO0 & Interrupt for communication between CC2500 and MSP430 \\
\hline GDO2 & Interrupt for communication between CC2500 and MSP430 \\
\hline SBWTCLK & Programming CLLK MSP430 \\
\hline SBWTDIO/RST & Reset signal to MSP430 / Programming pin \\
\hline TXD0 & Transmit Pin for Programming MSP430 \\
\hline RXD0 & Receive Pin for Programming MSP430 \\
\hline P17, P40, P47, P36, P10, P11, & \\
P13, P14, P15, P16, P41, P42 & General I/O - Extra Pin From MSP430 \\
P46, P25, P20, P21 & \\
\hline P22, P37, P23, P24 & Analog A2, A7, A3, A4 \\
\hline Gain Stage Out & Interface Board Split - Volume \\
\hline Q Gate & Interface Board Split - Volume \\
\hline I Gate & Interface Board Split - Volume \\
\hline P43 & Q Out - Volume \\
\hline P44 & I Out - Volume \\
\hline P45 & Pressure Out - Pressure \\
\hline P12 & Interface Module Enable \\
\hline &
\end{tabular}




\subsubsection{Vertical Connectors - Physical Construction:}

Molex micro-connectors [245] are chosen to serve as the vertical connectors in this prototype. These headers/receptacles are $1.54 \mathrm{~mm} \times 10.26 \mathrm{~mm}\left(15.8 \mathrm{~mm}^{2}\right)$ and have a mated height of $1.5 \mathrm{~mm}$ with a total of 44 pins at $0.4 \mathrm{~mm}$ pitch. The mated height of $1.5 \mathrm{~mm}$ is the minimum height to allow for proper clearance for ICs located above and below the specific module.

\subsection{PV Sensor Interface Module}

The Pressure-Volume (PV) Interface Module provides the stimuli and reference signals to the pressure and volume sensors located on the medical catheter. In return, it receives the raw PV signals from the catheter that is directly connected to the telemetry system by seven wires (4 for volume, 3 for pressure) using the common ground path, Fig. 4.11. The signal processing logic consists of pressure and volume sub-blocks, that is, each sensor's data is processed individually. The following subsections will describe the catheters used in this design, as well as both the pressure and volume circuits used.

\subsubsection{Catheters}

Catheters are an essential part of the wireless telemetry system as they provide the structure to house the bio-medical sensors along with a direct connection to the telemetry system. Catheters can contain various types of bio-sensors, in this research project, only two types of sensors are used, pressure and volume. These sensors can be separated into three catheter types: pressure only, volume only, and combined PV. This system can be used with any catheter type as each measurement block is computed individually, however, in this specific design PV catheters are used.

An example of a pressure-volume catheter used in this system is shown in Fig. 4.12. This diagram represents a 5 French catheter used for large animal research, with a diameter of 1.7 


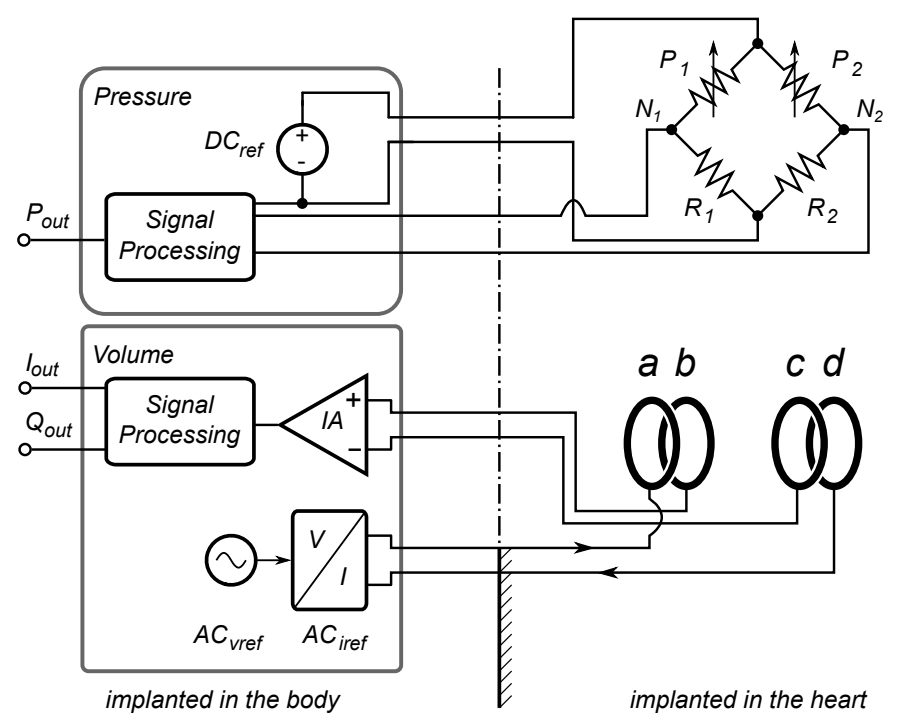

Figure 4.11: Simplified block diagram of PV interface module, and the equivalent electrical diagram of MEMS pressure and conductance based volume sensors themselves; (in reality, ground line is shared).

$\mathrm{mm}$. It contains four electrode rings and a pressure sensor separated at specific distances. The top and bottom sets electrodes are separated by a distance of $30 \mathrm{~mm}$ with each set separated by $3 \mathrm{~mm}$. Similarly, a 1.2 French catheter is used for small animal research with a diameter of $0.4 \mathrm{~mm}$, top and bottom ring seperation of $4.5 \mathrm{~mm}$, and each set separated by $0.5 \mathrm{~mm}$. The tetra-polar catheter is inserted directly into the test subject's left ventricle to measure blood pressure and volume. The two outer electrodes inject current into the blood and the two inner electrodes measure the voltage created by the electric field inside the left ventricle. Conductance/admittance is calculated from the known current and measured voltage. The blood pressure is directly determined from the included pressure sensor.

\subsubsection{Pressure Measurement}

The pressure measurement sub-block is used to convert the pressure created by the blood on the catheter (mmHG) to a voltage. This voltage is obtained by the use of a catheter containing a small MEMS based pressure sensor, Section 4.4.1 and is connected directly to the pressure 


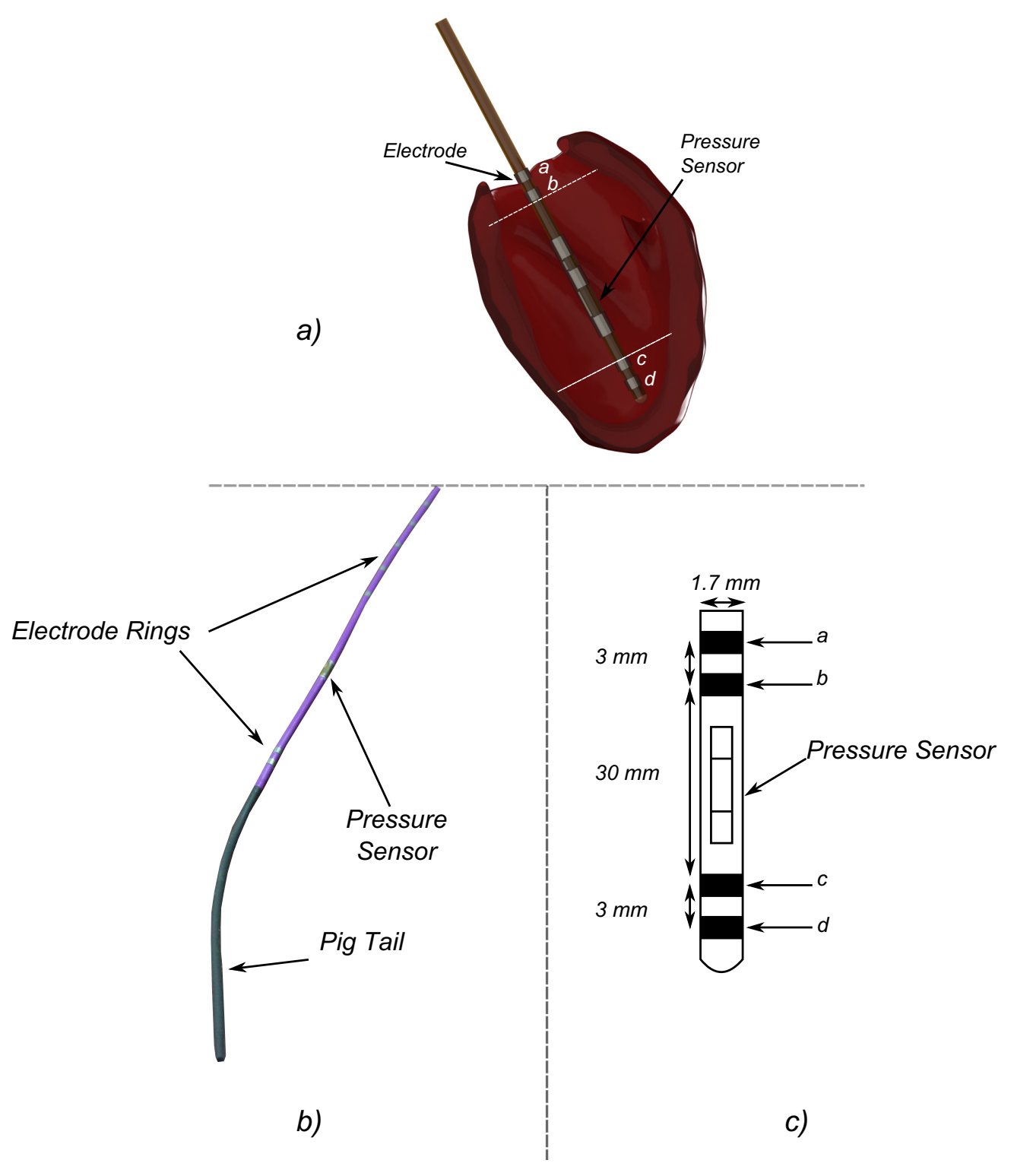

Figure 4.12: Pressure Volume catheter a) inserted into the LV of a heart; b) physical implementation of the $5 \mathrm{~F}$ catheter; and c) dimensions of the $5 \mathrm{~F}$ catheter. 


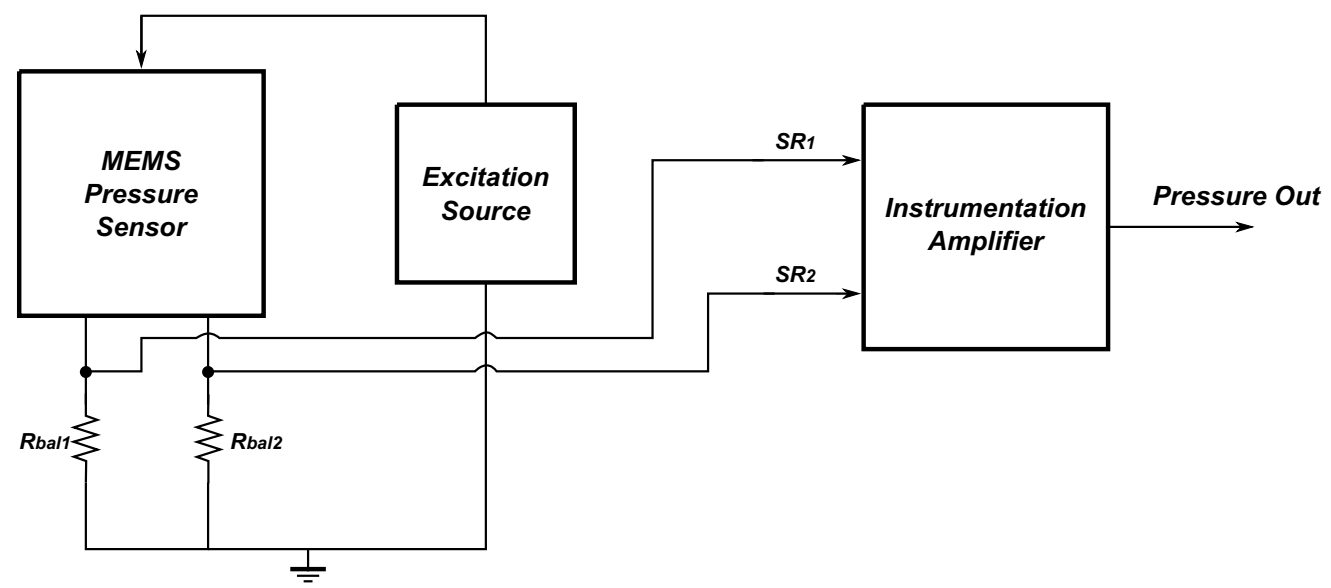

Figure 4.13: Traditional pressure measurement block.

block electronics for processing using three wires, SR1, SR2 and COM. These wires are used to excite the pressure sensor and provide voltage measurement, Fig 4.13. The specific pressure sensor used in this telemetry system is the GE P161 Pressure sensor [246]. This is a MEMS based sensor and contains two pressure sensitive resistors with a nominal resistance of $800 \Omega$. Two measurement techniques are used in this design: traditional wheatstone bridge, and digital IC with the digital IC version utilized in the in-vivo study.

\section{Traditional Wheatstone Bridge}

The wheatstone bridge is an combination of four branches of resistors used to measure an unknown resistance, by balancing two branches of the bridge circuit, with one branch including the unknown resistance, Fig. 4.11, top right. Nodes $N_{1}$ and $N_{2}$ represent the measurement points, the excitation signal is shown within the pressure processing block. The top two resistors, $P_{1}, P_{2}$, in the bridge represent the pressure sensor and the bottom two $R_{1}, R_{2}$, are external matching resistors. If the measured voltage between nodes $N_{1}$ and $N_{2}$ is zero then the bridge is balanced, in other words there is no pressure exerted.

When pressure is exerted on the sensor, the resistance of $P_{1}$ and $P_{2}$ will increase or decrease based on the difference in pressure from the calibrated pressure. The calibrated pressure is when the bridge is balanced i.e., no measured output voltage. The sensor resistor $P_{1}$ will 


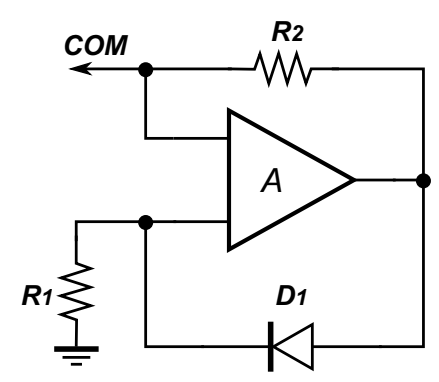

Figure 4.14: Current source design.

increase when $P_{2}$ decreases and vice versa, this causes the bridge voltage to be linearly based on the pressure exerted. The voltage difference is measured using a high gain instrumentation amplifier, typically $50-60 d B$ or higher and is directly proportional to the pressure exerted.

Drive Source: In most wheatstone bridge configurations the bridge is driven by a voltage source rather than a current source. In this system, a current source is used as it has various advantages. The first is that current driven wheatstone bridges have the advantage of not introducing measurement errors when the excitation circuit is far away from the sensor. In this telemetry system, the catheter is located away from the main electronics and could introduce measurement error due to the increased wire length and wire resistance if voltage driven circuits are used. The second benefit is the pressures sensitivity is directly linear to the excitation current, allowing the sensitivity to be controlled. Finally, the temperature at the sensor node can be measured and compensated for when using the current driven method. If using the voltage driven method an additional temperature sensor is required. A downside to using current driven bridges are the additional components and power consumption required to create a stable current source.

The current source is constructed using an OP-Amp, a diode and two resistors, Fig. 4.14 . This current source is located next to the interface electronics and is directly connected to the pressure sensor through the COM pin on the sensor. The constructed current source is designed to provide $\sim 580 \mu \mathrm{A}$ of current as required by the pressure sensor. The current source used is a simple diode and sense resistor configuration. The voltage drop of the diode across the resistor 
$\mathrm{R} 2$ provides the correct current to the sensor.

Compensation: The external resistors of the wheatstone bridge $\left(R_{1}, R_{2}\right)$ must be completed based on the specifications of the pressure sensor. As the pressure sensor contains two nominal $800 \Omega$ resistors, the bridge completion resistors must be close to $800 \Omega$ to balance the bridge correctly. The bridge completion resistors are generally tuned or trimmed to get the resistance needed to complete the bridge. Typically, exact $800 \Omega$ resistors are not used as some adjustment must be made to offset the effects of wire resistance, temperature, etc.

In most cases, sets of high precision resistors are connected in parallel to balance the bridge more effectively rather than single resistors. If the overall volume allows, analog potentiometers (POTs) are used to provide additional resistance trimming capabilities. However, these are larger in size and create additional noise. The benefits of using standard resistors or analog POTs are that they provide very accurate results as well as generally costing less and no additional ICs are used. The additional space increase for potentiometers and mechanical noise is a downfall of this method. As well, if only precision resistors are used no additional resistance trimming can take place once the circuit is constructed.

An improved method to trim the bridge completion resistors is to tune them digitally. Instead of a mechanical potentiometer that is much larger and cannot be tuned from outside of the implant, a digital potentiometer can be used. A digital potentiometer is a small IC, that contains several resistance steps from $0 \Omega$ to the maximum resistance and is controlled via SPI or $I^{2} C$. These POTs are generally connected with additional high precision resistors in parallel to get the exact bridge completion resistance. The digital POTs allow for constant resistive trimming to the bridge during operation. Downsides to using digital POTs over their analog counterparts are the additional area required for the IC packages (SOIC, MSOP, TSSOP, etc) and the additional power consumption and programming required. 


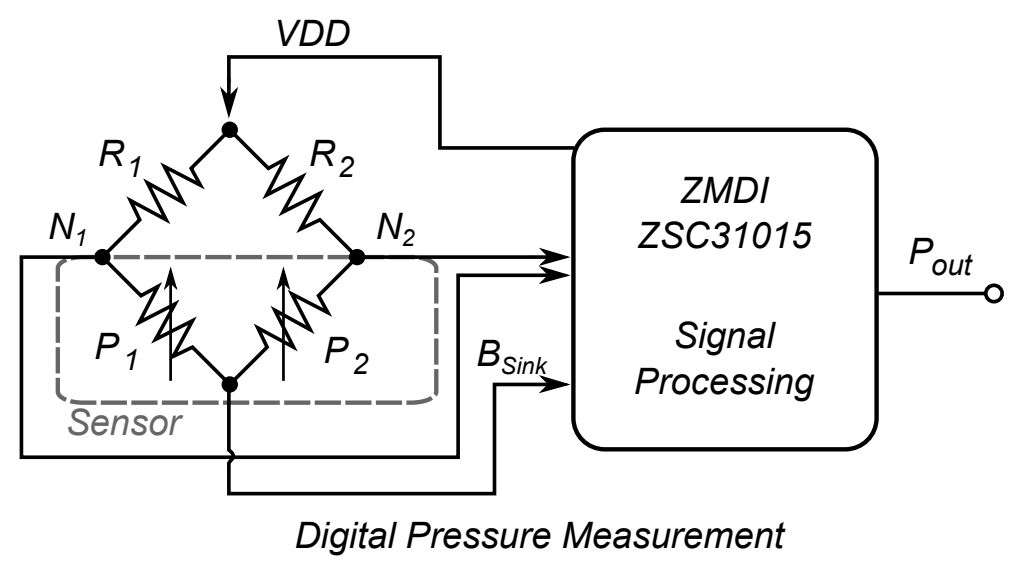

Figure 4.15: ZDMI ZSC 31015 based Digital Pressure Measurement Method.

\section{Digital Pressure IC}

An alternative to the traditional wheatstone bridge method is to use a digital IC to perform the excitation, sensing, and compensation to the bridge, Fig. 4.15. The ZMDI ZSC 31015 Digital pressure measurement IC, [28], is used in this design to perform this task. An internal block diagram of the ZSC 31015 is provided in Fig. 4.16. The basic functionality of the bridge remains the same, the bridge is excited, resistance changes based on pressure and a voltage is measured. Only three external components are required for the IC to operate: two bridge completion resistors and a single DC decoupling capacitor. When using this method, the bridge is voltage driven by $V D D$ rather than by a current source and will have the same drawbacks of traditional voltage driven bridges however, the ZSC 31015 calibrates these inaccuracies out during the calibration process. The instrumentation amplifier is located within the ZSC 31015 rather than as a separate component and its gain can be programmed. Furthermore, the ZSC 31015 provides onboard compensation including temperature (if external diode is used), gain and offset, etc. The measured voltage can be output as a ratio from $0-1 \mathrm{~V}, 0-V D D$, or digitally via the ZMDI custom one wire interface. In this system 0 to $V D D$ is chosen as it allows the remaining components of the system(wiring, ADC, firmware) to remain unchanged if a traditional wheatstone bridge configuration is used. The COM pin from the catheter is connected to the $B_{\text {Sink }}$ pin of the ZSC 31015 to provide additional power savings. The $B_{\operatorname{Sink}}$ 


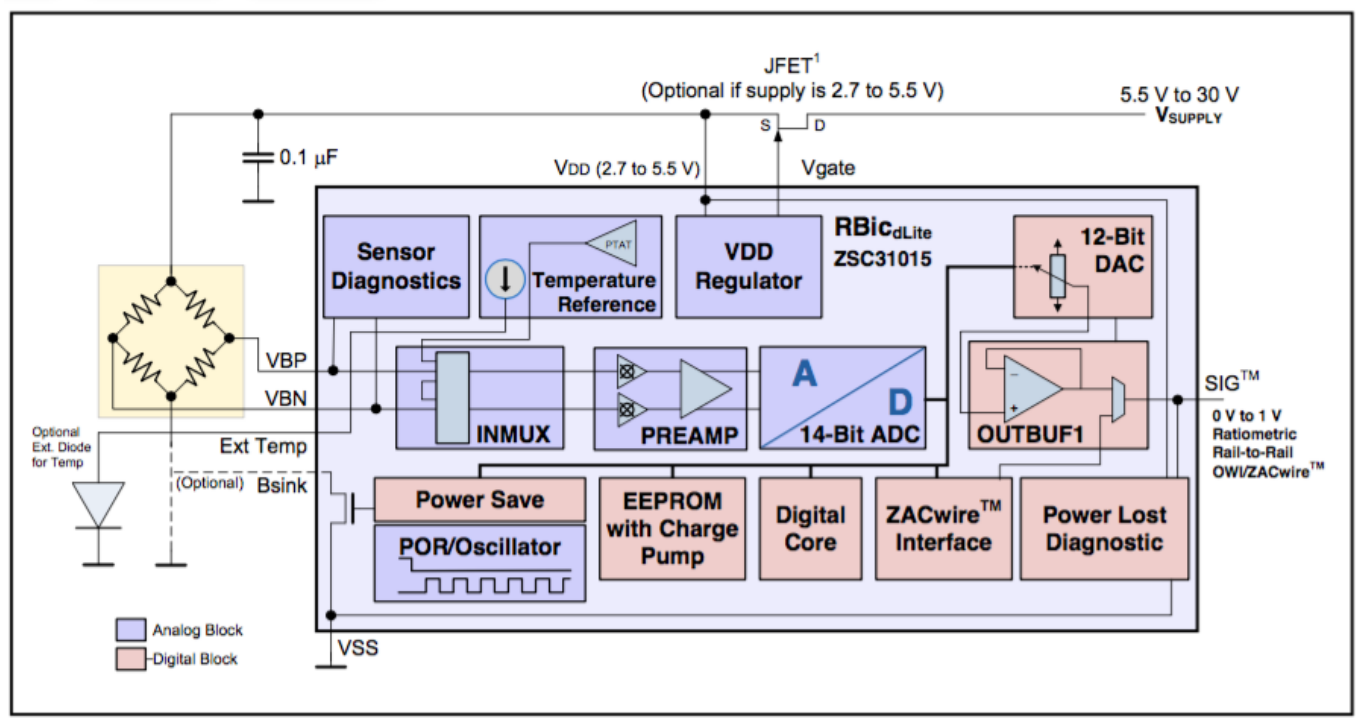

Figure 4.16: ZDMI ZSC 31015 Internal Block Diagram [28]. Copyright (cZMDI.

pin is connected to an internal switched transistor to interrupt the bridge current when not sensing. The switch is synchronized to the internal ADC of the IC and is only opperational during conversion. The power consumption savings is dependent on the sample rate of the $\mathrm{ADC}$ and can reduced from $20 \%$ to $1 \%$, typical current consumption is $250 \mu \mathrm{A}$. Additional features include 24-bit identification, and the ability to be used in die form. These features are not used in the current implementation of the system.

The ZSC 31015 is configured using a separate programming module provided by ZMDI. Once connected, all features of the IC can be configured, including gain, sample rate, offsets, calibration, etc. Section 5.3.1 provides details into the pressure configuration and calibration process for the implantable telemetry system. The calibration data is stored in EEPROM and recalled into memory upon on power-up.

By using this digital IC in the pressure measurement block all the disadvantages of traditional approach is avoided or can be calibrated out. Additional control is also gained in the signal conditioning chain and if connected digitally, can be programmed in test for better programability. Although, this method has great advantages, the fact that additional hardware and software are required to program and setup the device is a disadvantage and adds complexity 
to the design over simple mechanical POTs. However, the signal resolution and multi-point calibration enable much higher quality signals to be measured and as a consequence provide improved results over the traditional wheatstone setup.

\subsubsection{Volume Measurements}

The volumetric section of interface uses the admittance methodology to measure the blood volume in the $\mathrm{LV}$, that is, both magnitude and phase of the measured voltage are extracted. The volumetric sensor itself (i.e. the four rings $(\mathrm{a}, \mathrm{b}, \mathrm{c}, \mathrm{d})$ ) is excited by voltage reference $A C_{\mathrm{vref}}$ that is converted into current reference $A C_{\text {iref }}$, Fig. 4.11. Phase sensitive detectors along with a quadrature oscillator are used to extract DC In Phase and Quadrature voltages (I and Q) that are proportional to the raw in phase and quadrature voltage signals at the PV sensor. The I and Q signals are sampled by the microcontroller and transmitted to the external device. Further processing is required to convert these signals into calibrated magnitude and phase values and used to compute the volume signal and will be discussed in Sections 4.8 .

In the following subsections the background theory on lock-in amplifiers will be introduced, followed by circuit implementation details of the volume interface, Section 4.4.3.

\section{Lock-In Amplifier Background Theory}

Traditional approaches in measuring the admittance signal in the LV rely on active bandpass filters to reduce noise from the measurement signal, however, any noise not fully suppressed impacts the magnitude measurement. An alternative technique to measure admittance is to use phase sensitive detectors also known as Lock-In Amplifiers. Furthermore, this improved technique allows for the direct extraction of the phase signal where as traditional approaches require additional hardware and pulse width analysis to determine the phase.

Lock-in amplifiers are used to measure low voltage AC signals ( $\mu \mathrm{V}$ to $\mathrm{nV}$ ) within noisy environments (e.g., animal body) and amplify them to produce a useable signal. These noise signals can be many times larger than the signal of interest and a lock-in amplifier is still able 


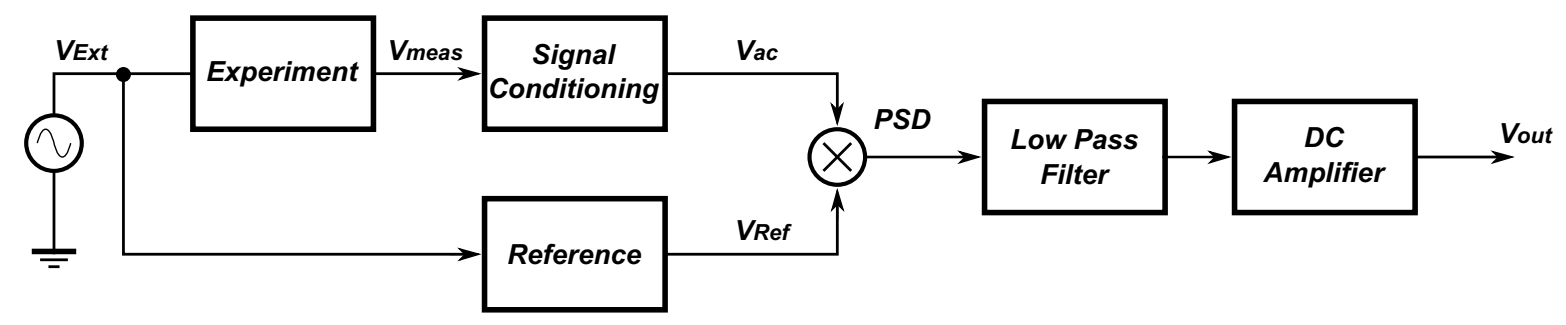

Figure 4.17: Block diagram of a Lock-In Amplifier.

to extract such a signal [247]. Recall, standard AC voltage measurements measure all voltages at the measurement input, including all noise signals as well as the signal of interest. This can cause signals of interest to be lost or overwhelmed with noise. Lock-in amplifiers are different from these $\mathrm{AC}$ voltage measurements because not only is a excitation signal provided to the experiment but a reference signal is also provided [248]. This signal must be phase and frequency locked with the original excitation signal for the amplifier to work correctly. The lock-in amplifier uses the reference signal to locate the measured signal of interest within the noisy environment, while disregarding all signals that are not of the same frequency as the reference signal. This technique is known as phase sensitive detection [249]. This is the main reason that a lock-in amplifier can measure very small experimental voltage amplitudes in an environment that contains noise signals much greater. Lock-in amplifiers are used in many different applications from gas monitors [250] to wireless embedded applications [251-253].

The basic lock-in amplifier structure, Fig. 4.17, contains seven main components: excitation source, experimental system, signal conditioning, reference generation, phase sensitive detector (PSD), low pass filter (LPF), and DC amplifier [248].

Time Domain Analysis: The excitation signal $V_{e x t}(t)=V_{o} \cos \left(2 \pi f_{o} t\right)$ is fed into the test system to initiate the measurement. The measured signal is first directed through the signal conditioning block. Here, the measured waveform is amplified and filtered to remove unwanted frequencies above and below the frequency of interest, $f_{o}$. The output of the signal conditioning block is (4.2), where $G_{a c}$ is the gain of the signal conditioning block, $V_{o}$ is the amplitude of the signal of interest, $f_{o}$ is the excitation frequency, and $\theta_{s}$ is that possible phase shift of the signal. 


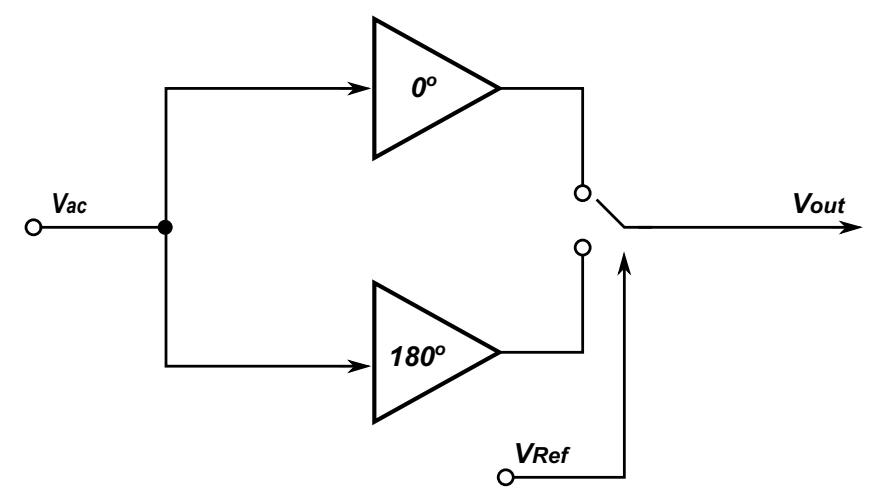

Figure 4.18: Ideal mixer implementation.

This represents one input to the PSD.

$$
V_{a c}(t)=G_{a c} V_{o} \cos \left(2 \pi f_{0}+\theta_{s}\right)
$$

The phase sensitive detector is a specific type of frequency mixer (multiplier). A frequency mixer in the most basic sense multiplies two signals $V_{1}(t)$ and $V_{2}(t)$ to form a new signal. As stated previously, one input is 4.2 and the reference voltage, $V_{r e f}(t)$, is the other. A mixer can be implemented in many forms including transistors, diodes, or any non linear device. However, in a simple implementation, the mixing circuit can be described by Fig.4.18[251]. Here, the measured signal, (4.2), is split into the in-phase $0^{\circ}$ and the anti-phase $180^{\circ}$ components, where a switch controls the output. This switch is controlled by the reference signal, $V_{r e f}(t)$. This configuration offers the widest dynamic range to maximize the signal recovery [249].

The reference signal is chosen to be a square wave with a gain of 1 to control the switch in the PSD. In different mixer circuit configurations the reference signal can be a general sinusoid. The reference signal is phase and frequency locked to the original excitation frequency $V_{\text {ext }}(t)$ and is represented by 4.3 .

$$
V_{r e f}(t)=\frac{4}{\pi}\left[\cos \left(2 \pi f_{o} t+\theta_{R}\right)-\frac{1}{3} \cos 3\left(2 \pi f_{o} t+\theta_{R}\right)+\frac{1}{5} \cos 5\left(2 \pi f_{o} t+\theta_{R}\right) \ldots\right]
$$


When two signals of frequencies $f_{1}$ and $f_{2}$ are multiplied together, two additional signals are created with one equal to $f_{1}+f_{2}$ and another equal to $f_{1}-f_{2}$, along with harmonics. Therefore if (4.2) and (4.3) are multiplied (4.4) is created, where $\omega_{R}=2 \pi f_{\text {ref }}, \omega_{S}=2 \pi f_{\text {meas }}$ and $\theta_{R}, \theta_{S}$ represent the phase shifts of the respective signals.

$$
\begin{aligned}
V_{P S D}(t) & =\frac{2 \sqrt{2} V_{o} G_{a c}}{\pi}\left[\cos \left(\omega_{R} t \pm \omega_{S}+\theta_{R} \pm \theta_{S}\right)\right. \\
& -\frac{1}{3} \cos \left(3 \omega_{R} t \pm \omega_{S}+3 \theta_{R} \pm \theta_{S}\right) \\
& \left.+\frac{1}{5} \cos \left(5 \omega_{R} t \pm \omega_{S}+5 \theta_{R} \pm \theta_{S}\right) \ldots\right]
\end{aligned}
$$

If the assumption that the measured signal and reference signal are frequency locked (i.e., $\left.\omega_{R} t=\omega_{S} t\right)$ is made and that the subsequent low pass filter cuts off well below the reference frequency (i.e., removes upper harmonics) then (4.4) reduces to 4.5).

$$
V_{P S D}(t)=\frac{2 \sqrt{2} V_{o} G_{a c} G_{l p f}}{\pi} \cos \left(\theta_{R}-\theta_{S}\right)
$$

Equation 4.5 is directly proportional to the amplitude of the signal of interest $V_{o}$ along with a phase difference of $\theta_{R}-\theta_{S}$. However, if the experiment does not introduce a phase shift, then this component can be ignored. In addition, the LPF block acts as an integrator to remove any additional noise created at the output of the mixing circuit. Further DC gain can be obtained through a DC amplifier. All factors are known except for $V_{o}$, therefore, the measured signal can be extracted from the noisy environment using the final DC output, (4.6).

$$
V_{P S D}=\frac{2 \sqrt{2} V_{o} G_{a c} G_{l p f} G_{D C}}{\pi}
$$

Magnitude and Phase Measurements: The previous lock-in amplifier design will detect the magnitude of the measured signal only when $\theta_{R}-\theta_{S}=0^{\circ}$. If phase change occurs, the above system cannot measure it. Four examples are provided that illustrate the difference in the output waveform $\mathrm{V}_{P S D}$ when: a) there is no phase shift present between the reference signal $\mathrm{V}_{\text {ref }}$ and the input signal $\mathrm{V}_{a c}$, Fig. $4.19 \mathrm{a}$; b) $180^{\circ}$ out of phase, Fig. $4.19 \mathrm{p}$; c) $90^{\circ}$ out of phase, 
Fig. 4.19k; and d) $60^{\circ}$ out of phase. The output of the low pass filters in each example is not shown and is the mean of the $\mathrm{V}_{P S D}$ signal. Furthermore, if the amplitude of the input signal $V_{a c}$ changes, increase or decrease, while $\mathrm{V}_{r e f}$ and $\mathrm{V}_{a c}$ are in phase, the output of the LPF will change accordingly. However, if the amplitude and a phase shift occur $\left(\theta_{R}-\theta_{S} \neq 0^{\circ}\right.$.) at the same time it is impossible to distinguish the effect the original amplitude change vs phase shift's effect on $\mathrm{V}_{P S D}$ at the output of the LPF. This is due to the mean of $\mathrm{V}_{P S D}$ changing in both cases. By viewing $\mathrm{V}_{P S D}$ 's shape it can easily be seen if a phase shift is occurring, just not measurable at the output of the LPF. Thus, a different method is required to measure both the magnitude change and the phase change at the same time.

To measure the phase shift created by the experimental process, two phase sensitive detectors are required [249] [247], Fig. 4.20, The second PSD requires a reference signal shifted by $90^{\circ}$ from the original excitation signal. This allows quadrature signals to be measured or In Phase and Quadrature components (I and Q). The second PSD's output is (4.7).

$$
V_{P S D 2}(t)=\frac{2 \sqrt{2} V_{o} G_{a c} G_{l p f} G_{D C}}{\pi} \sin \left(\theta_{R}-\theta_{S}\right)
$$

By using equation (4.5) and (4.7) the magnitude and phase of the measured signal can be calculated using simple trigonometry. The magnitude is calculated by (4.8) and the phase is calculated by (4.9).

$$
\begin{gathered}
\text { Magnitude }=\sqrt{\left(V_{P S D 1}\right)^{2}+\left(V_{P S D 2}\right)^{2}} \\
\text { Phase }=\tan ^{-1}\left(\frac{V_{P S D 2}}{V_{P S D 1}}\right)
\end{gathered}
$$

Using this technique the magnitude and phase of a specific signal can be measured inside a noisy environment. 


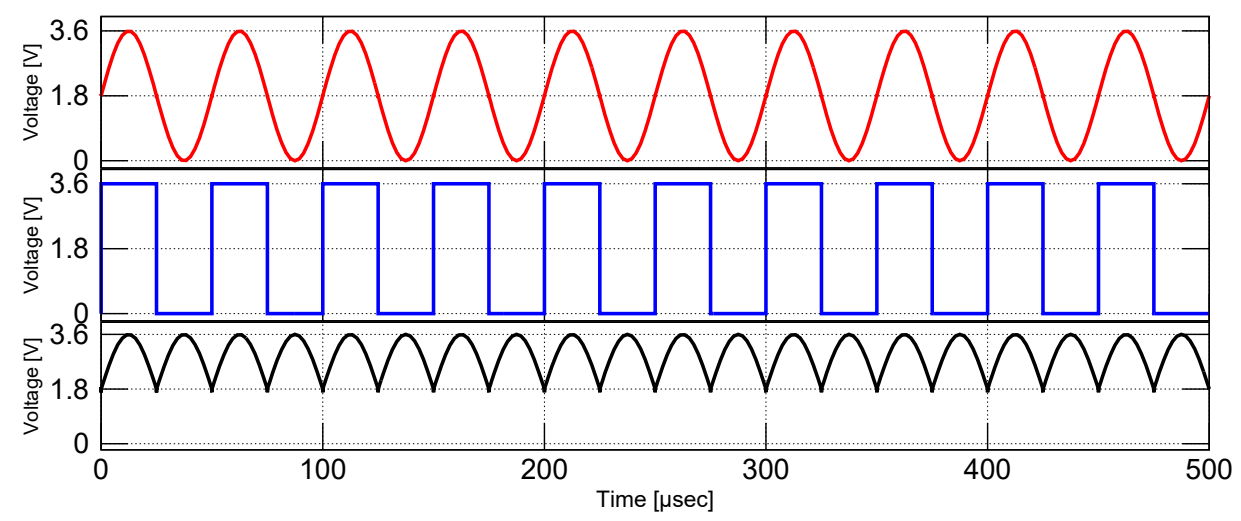

(a)

$V_{a c}$ and $V_{\text {ref }}$ in phase

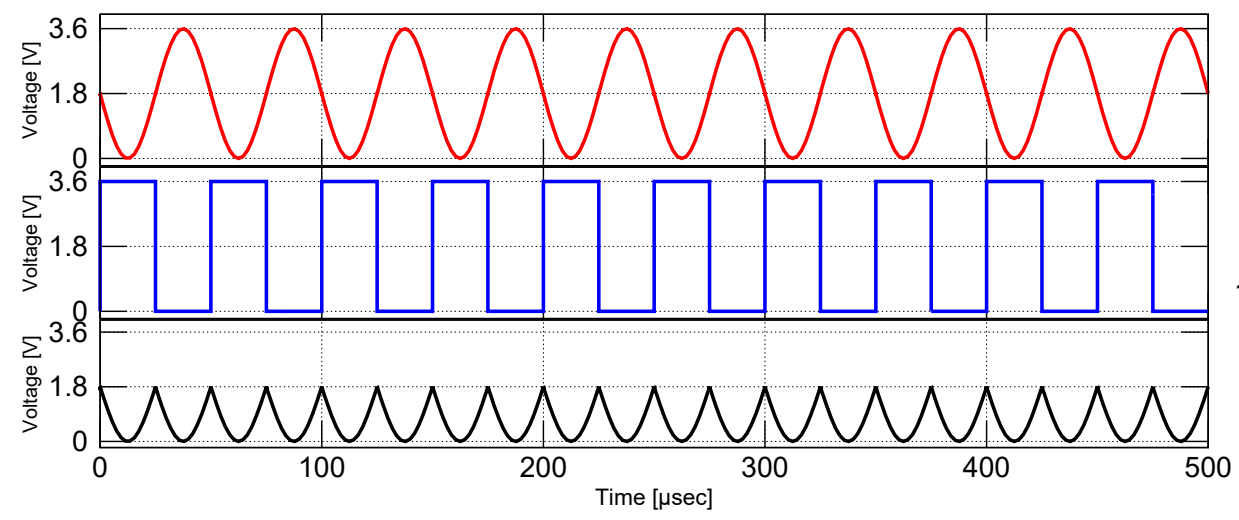

(b)

$V_{a c}$ and $V_{\text {ref }}$ $180^{\circ}$ out of phase

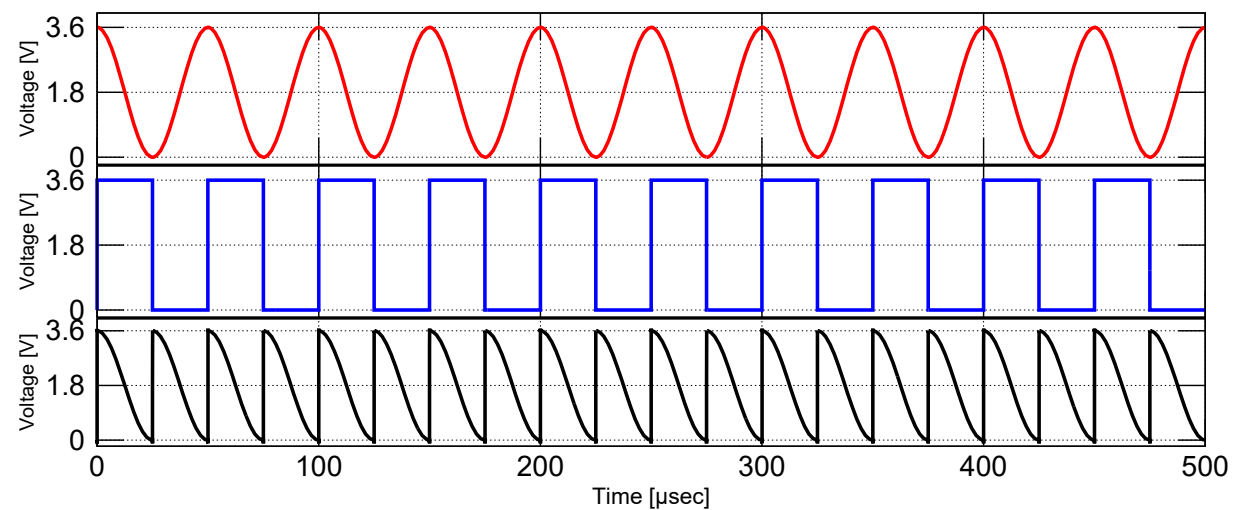

(c)

$V_{a c}$ and $V_{\text {ref }}$ $90^{\circ}$ out of phase

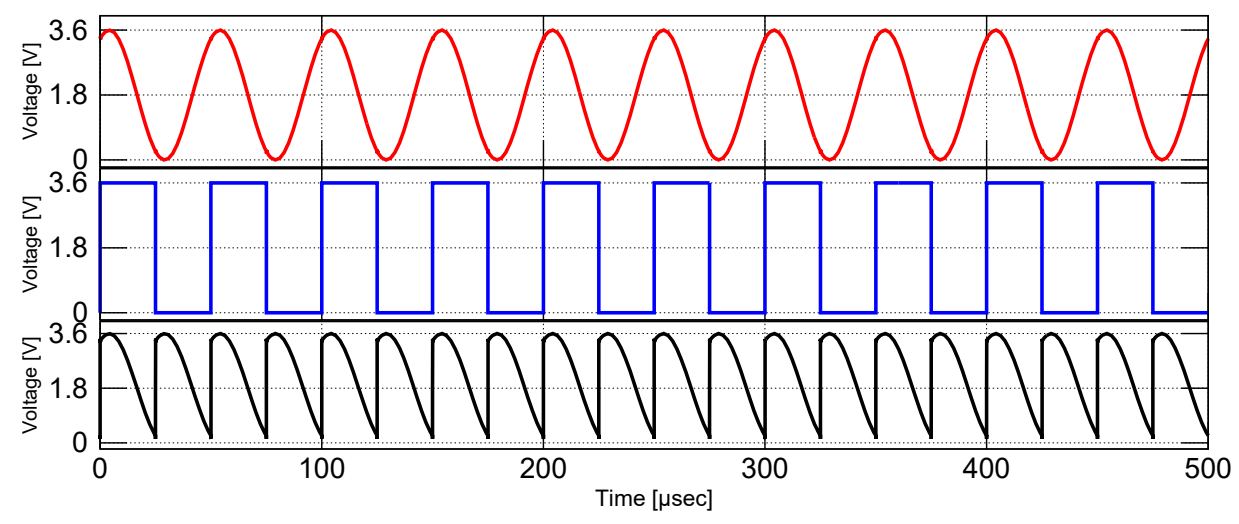

(d)

$V_{a c}$ and $V_{\text {ref }}$ $60^{\circ}$ out of phase

Figure 4.19: Phase Sensitive detector examples where the output $\mathrm{V}_{P S D}$ (black) is shown with $\mathrm{V}_{a c}$ (red) and $\mathrm{V}_{\text {ref }}$ (blue) at different phase shifts: a) in phase; b) $180^{\circ}$; c) $90^{\circ}$; and d) $60^{\circ}$. The output of the low pass filter is not shown. 


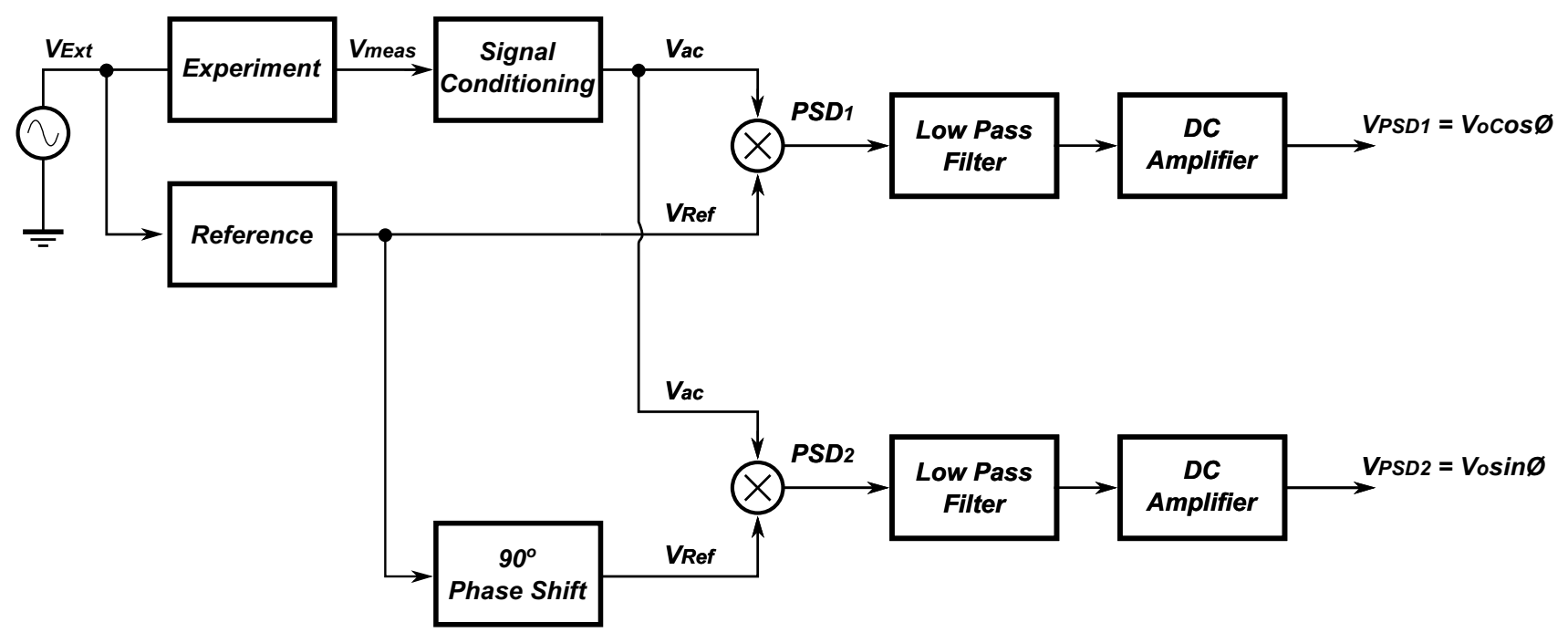

Figure 4.20: Dual Lock-In Amplifier block diagram.

\section{Lock-In Amplifier Circuit Implementation}

The circuit implementation of the volume interface is realized by the block diagram in Fig. 4.21 where nine stages are used to excite the catheter and extract the DC In Phase and Quadrature voltages to calculate the complex admittance. The outputs of the volume interface $(I, Q)$ are connected via the communication bus to the microcontroller to be sampled. Each stage's implementation is described below.

Quadrature Oscillator: A quadrature oscillator is designed, Fig. 4.22 to generate sine and cosine signals at $19.866 \mathrm{kHz}$ centred around the virtual ground of $1.8 \mathrm{~V}$. As discussed in Section 2.3.2, exciting the heart with a frequency of $\sim 20 \mathrm{kHz}$ causes the blood to become purely resistive and the heart muscle to contain both resistance and capacitance properties, allowing only the blood volume to be measured. This implementation uses six passive components (three $820 \mathrm{pF}$ capacitors and three $9.76 \mathrm{k} \Omega$ resistors) and two operational amplifiers. Other oscillator configurations were researched such as phase shift and Wien Bridge oscillators [254] however to achieve the required frequency, dual output ( $\sin$ and $\cos$ ) and full voltage swing required more discrete components to create the equivalent circuit.

High slew rate amplifiers are needed to achieve the required frequency and switching speed 


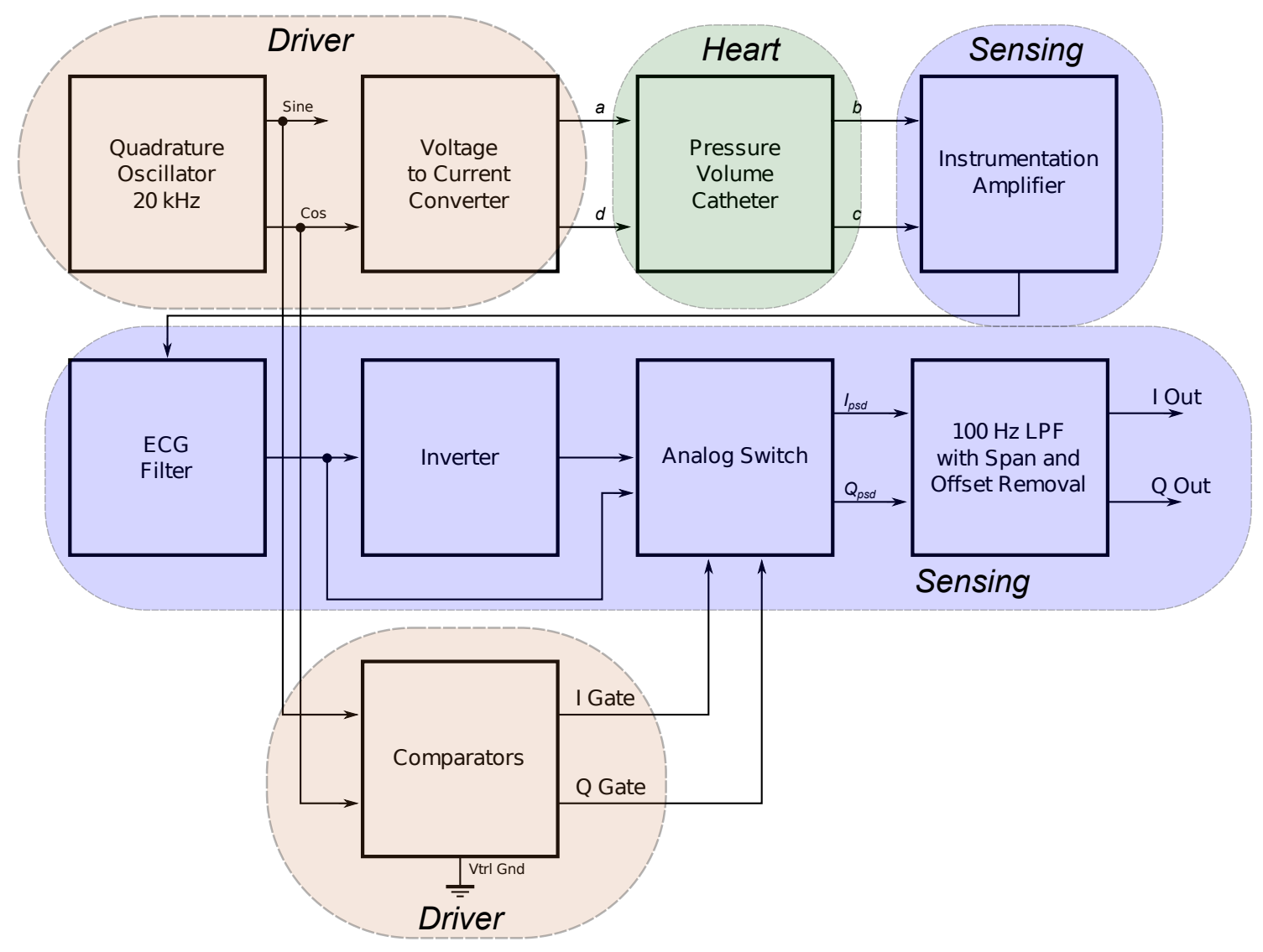

Figure 4.21: Block Diagram of Volume Measurement Processing. 


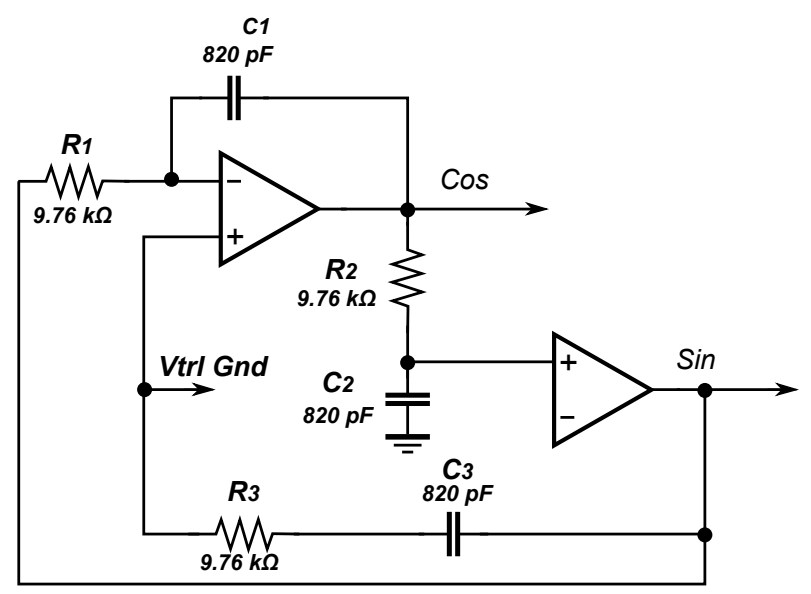

Figure 4.22: Quadrature Oscillator (outputs sine and cosine signals) designed with a $F_{0}=20$ $\mathrm{kHz}$.

of the oscillator. Texas Instruments LMV554 Op-Amps are chosen for this design as they provide a high gain bandwidth product (GBP) of $3 \mathrm{MHz}$, a slew rate of $1 \mathrm{~V} / \mu \mathrm{s}$, high rejection ratios (CMRR, PSRR), and still having low supply current of $37 \mu \mathrm{A}$. Additional Op-Amps were tested providing either very low power sub-100 $\mu \mathrm{A}$ or high GBP $(5 \mathrm{MHz})$ but not both. The LMV554 is used as the Op-Amp in all remaining designs.

Reference Generation: The reference signals for the analog multiplier are created using two comparators. The inputs to these comparators are the generated sin and cos waveforms and the virtual ground. To reduce component count Op-Amps are used in open loop configuration rather than separate dedicated comparators. The resulting output signals are frequency locked square waveforms providing a phase reference for the excitation signal and are connected to the analog switch.

Voltage to Current Converter: The in phase or cosine signal is used to directly excite the heart through a voltage to current (V-to-I) converter. This V-to-I converter uses three resistors $(20 \mathrm{k} \Omega, 12 \mathrm{k} \Omega$, and $1 \mathrm{M} \Omega)$ and one operational amplifier to achieve $61 \mu \mathrm{A}$ of alternating current, Fig. 4.23. This current is calculated by (4.10). Additional DC offset compensation is achieved through resistor $R_{5}$. The two input rings on the catheter (a,d) are connected to the 


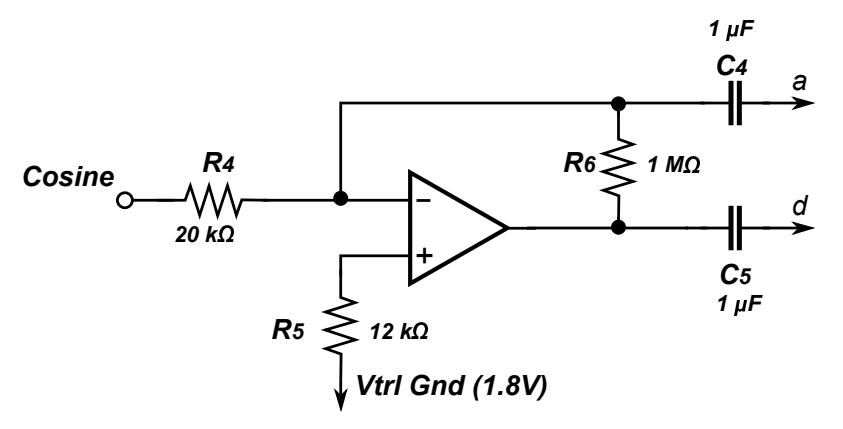

Figure 4.23: Voltage to Current converter implemented.

output of the V-to-I converter through two $1 \mu \mathrm{F}$ DC blocking capacitors and a $1 \mathrm{M} \Omega$ resistor separating the two nodes of the circuit. This excitation current generates an electric field between the two outer rings (a,d), Fig. 4.11, enabling a voltage to be measured.

$$
\begin{aligned}
& I=\frac{V_{r m s}}{R} \\
& I=\frac{1.22 V_{r m s}}{20 \mathrm{k} \Omega} \\
& I=61 \mu \mathrm{A}
\end{aligned}
$$

Signal Conditioning: The voltage created by the electric field through the LV is measured by an instrumentation amplifier (IA), Fig 4.24. Two $10 \mathrm{nF}$ capacitors are used to decouple any DC offset created from the electrode rings during measurement. In addition, two $100 \mathrm{k} \Omega$ resistors are used to restore the virtual ground offset to the incoming AC signal. The voltage difference is amplified with a gain of $20 d B$ by the IA. The IA used in this design is the TI INA333, with low current consumption of $50 \mu \mathrm{A}$ and high CMRR of $100 \mathrm{~dB}$. A benefit of using this IA over other options is its high accuracy, low power and single gain resistor required. However, it cannot provide high gain, greater than $20 \mathrm{~dB}$, at and above $20 \mathrm{kHz}$. As a result, the gain of the IA must be kept at $20 d B$ and an additional gain stage of $17 d B$ is used for a total stage amplification of $37 \mathrm{~dB}$.

Along with the desired voltage waveform the electrode rings will also measure the electrical signals created from the surrounding heart nerves and muscles causing additional unwanted 


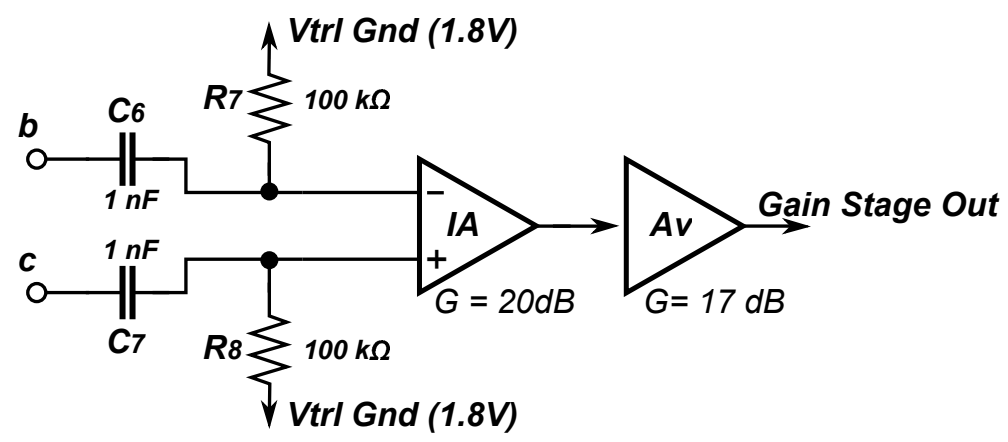

Figure 4.24: Instrumentation Amplifier and Gain Stage configuration.

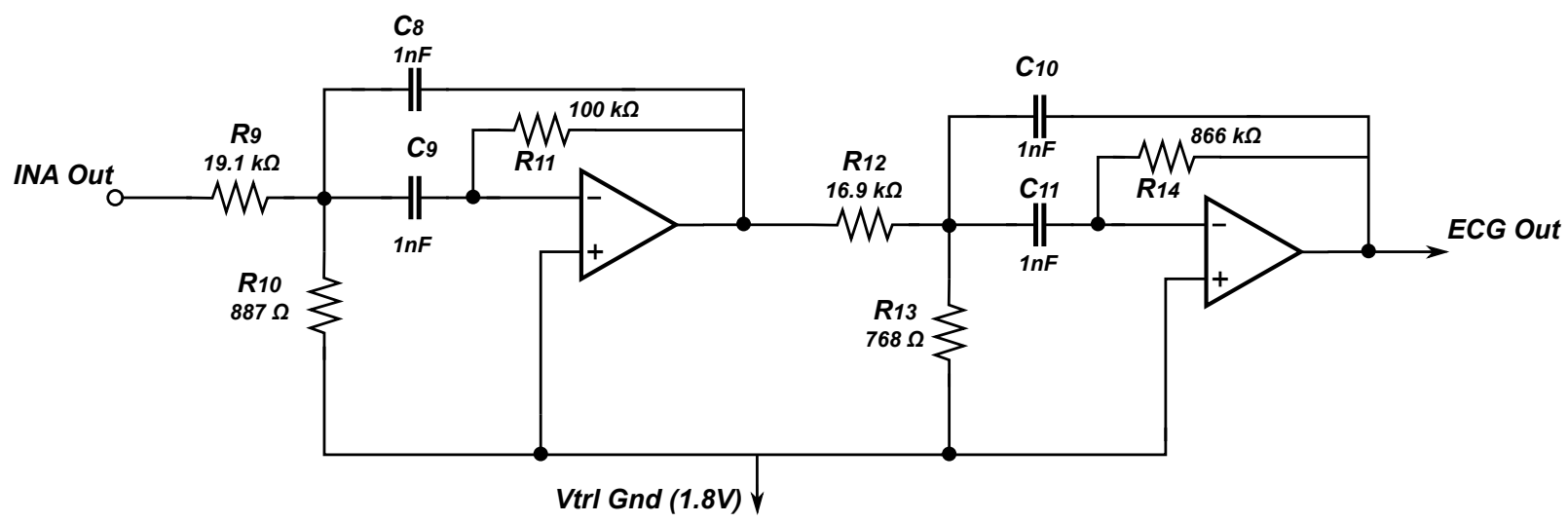

Figure 4.25: ECG bandpass filter to remove ECG signals measured from surrounding heart nerves.

noise in the signal. A bandpass filter is used to remove the ECG signal and additional noise contained in the measured AC signal. A $4^{\text {th }}$ order, two stage multiple feedback bandpass filter is designed with a total gain of $12.04 \mathrm{~dB}$ and is centred around $18.59 \mathrm{kHz}$ with a passband of $4 \mathrm{kHz}$. A total of 10 passive components (6 resistors and 4 capacitors) and two OP-Amps are used to implement this filter, Fig. 4.25. This amplified and filtered signal is the main input to the next stage of lock-in amplifier - the phase sensitive detector.

Mixer: Two main blocks of the phase sensitive detector are the mixer and low pass filter. As mentioned previously one of the simplest forms of a mixer is an inverter/switch combination, Fig. 4.18. The designed interface module implements this mixer configuration and an active low pass filter design. The inverter block is implemented by an unity gain inverting amplifier 


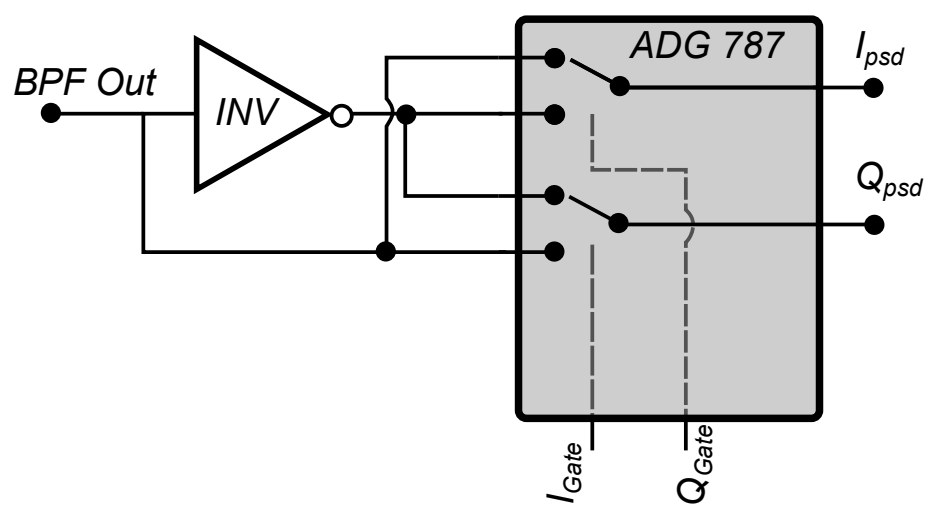

Figure 4.26: Inverter and analog switch, represents the phase sensitive detector in the lock-in amplifier.

configuration, Fig. 4.26. To extract both I and Q values, two analog switches are needed, the Analog Devices ADG787 is used to complete the mixer configuration. The input to each switch is the inverted and the non-inverted filtered signal with the frequency locked square waves providing the switching controls, Fig. 4.26, $I_{P S D}$ and $Q_{P S D}$ are the outputs of the switch.

Span and Offset: The high frequency harmonics of the output waveform are removed using a low pass filter to reduce the waveform to a DC level that is directly proportional to the magnitude and phase of the voltage signal. To obtain maximum SNR, the DC signal must span the full input range of the microcontroller $(0-3.6 \mathrm{~V})$. This is achieved by removing the virtual ground offset of $1.8 \mathrm{~V}$ and amplifying the DC output to span between 0 and $3.6 \mathrm{~V}$. A $2^{\text {nd }}$ order Bessel active low pass filter with a cut-off frequency of $127 \mathrm{~Hz}$ is designed with a gain of 13.46 $d B$ and resistors to remove the DC offset, Fig. 4.27. Each switch output, $I_{P S D}$ and $Q_{P S D}$, is connected to a separate active low pass filter block, each with separate offsets $(R 18$ and $R 19$ are swapped from what is shown in Fig. 4.27 for Q out LPF) to achieve maximum scale for both I and Q outputs.

The outputs, I and Q, are connected to the communications bus and sampled by the MSP430. The I and Q signals are sent to the external device with no additional processing. Calibration, admittance, and volume calculations are done on the external device. More information on this 


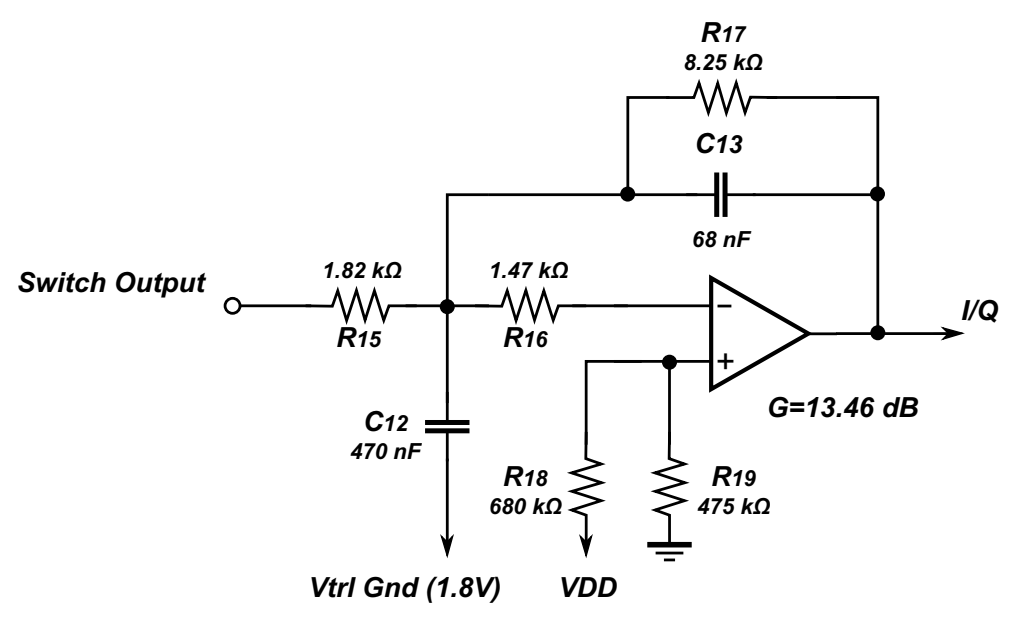

Figure 4.27: $127 \mathrm{~Hz}$ Low Pass filter with gain and span included.

is outlined in Section 4.8 ,

\subsubsection{Interface Module - Implementation}

The physical implementation of the interface module consists of the pressure signal conditioning, volume measurement blocks and catheter attachment pads. Due to the large component count of the volume measurement electronics the PCB area will be large. Rather than extend the area of a single printed circuit board it is more beneficial to extend the design to two separate PCBs. This increases the overall volume slightly however, it does not change the shape of the system, as it still remained cubic shaped. Design decisions are made on how to separate the design across two PCBs, this includes which signals are connected to the communications bus, which packages are used, and where the catheter pads are located. In addition, this module's power supply (LDO on the power module, $3.6 \mathrm{~V}$ and $1.8 \mathrm{~V}$ ) is controlled via the microcontroller.

The catheter attachement pads are located on the top PCB board and are $1.5 \mathrm{~mm} \times 1.5 \mathrm{~mm}$ to allow for easy mounting of the catheter. As mentioned above the TI LMV554 is used as the OP-Amp in this design and is packaged in either a TSSOP14 (Quad Op-Amp) or MSOP8 (Dual Op-Amp). The TI INA333 is packaged in a no lead package measuring $3 \mathrm{~mm}$ x 3 


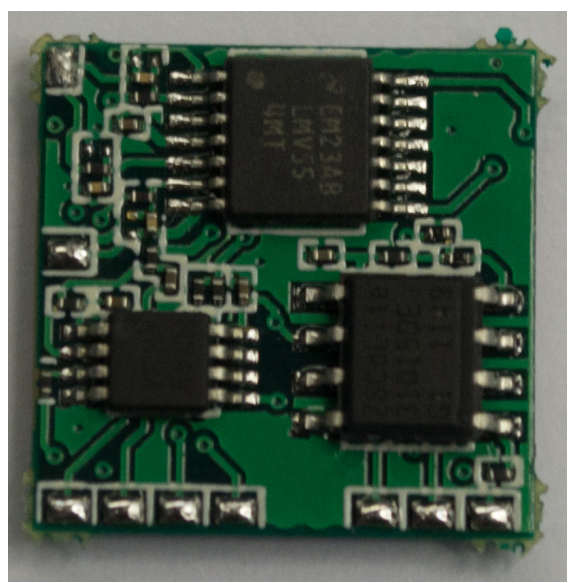

Top Interface Board

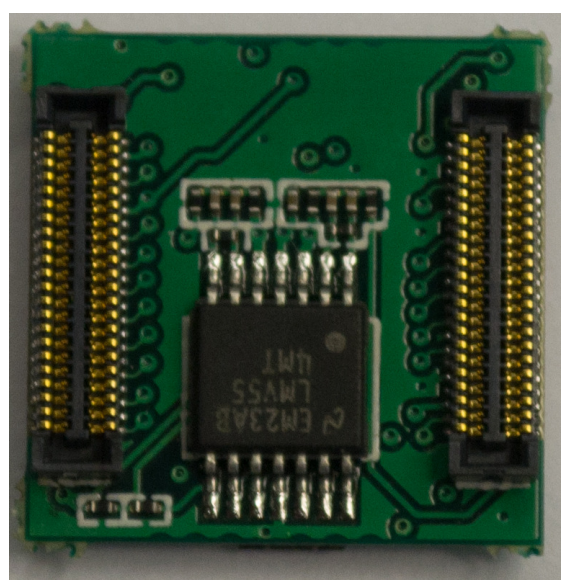

Inner Interface Board

Figure 4.28: Interface Module - Pressure and Volume PCB: Top PCB, left, and right inner PCB.

mm. All passive components are 0201 size and the remaining analog switch is a MSOP10 package. The pressure module consists of a few passive components and the ZMDI ZSC31015 is packaged in a SOIC8 which is the largest component in the interface module. A die version is available however, it is not used at this time.

As interface module is split across two PCBs, multiple signals are to be routed to the communication bus to be connected to the second PCB in the stack. When choosing which signals are to be connected, the physical packages of the components are considered. In this design, there are a total of 11 OP-Amps, 1 IAs and 2 switches that are used by the volume electronics and a single ZMDI ZSC31015 in the pressure section. A single Op-Amp SOT23 package must be used in order to achieve the exact total number of Op-Amps required for this design. However, this extra component will add a significant amount of area and volume to the design (pads, height, etc). Alternatively, the physical amount of Op-Amps placed on the PCB is increased by 1 such that the OP-Amps are split up into different combinations of quad and dual packages (i.e., 3 quads or 2 quads and 2 duals, etc). The extra Op-Amp is left unconnected on the PCB. Therefore, taking these considerations and analyzing the schematic to view logical separation points it is determined that three signals are used for separation purposes, $\mathrm{I}_{\text {Gate }}, \mathrm{Q}_{\text {Gate }}$ and the output of the gain stage after the IA. $\mathrm{I}_{\text {Gate }}$ and $\mathrm{Q}_{\text {Gate }}$ represent the output of the comparators 
to create the Lock-In reference signals for the I and Q signal chains. In addition, all pressure components are on placed on single $\mathrm{PCB}$ and the output pressure sent to the communication bus.

Using these three signals along with output pressure, the interface module is split into two PCBs created using a total of seven packages. The first PCB contains four packages: 1 quad OP-Amp, 1 dual OP-Amp, a single instrumentation amplifier, and the ZMDI ZSC31015 IC. The second PCB contains three packages: 1 quad OP-Amp, 1 dual OP-Amp and 1 dual analog switch. The complete pressure section is located on the top PCB along with the catheter pads. The second PCB contains the outputs I and Q which are connected to the microcontroller through the communications bus. The two PCBs are fabricated on $15 \mathrm{~mm}$ x $15 \mathrm{~mm}$ FR-4 boards and shown in Fig, 4.28 for the first and second board.

\subsection{Testing Platform}

As the implant's physical size is small and it is not practical to probe the internal boards while in use, a test platform is developed to allow for full programming, charging, and debugging of the wireless telemetry system. This system consists of two PCBs, a breakout board and a full featured development board.

\subsubsection{Breakout Board}

The breakout board's main purpose is to route all the signals from the communication bus to two additional connectors. These connectors are located on the opposite ends of the board compared to the vertical communication bus, Fig.4.29(a). Flat flex connectors (FFC) are chosen for the breakout connectors, more specifically 26 pin $0.5 \mathrm{~mm}$ pitch Molex FFC connectors [255]. These are right angle flat cable connectors with a mated height of $2 \mathrm{~mm}$. The overall PCB size is increased to $25 \mathrm{~mm} \times 21 \mathrm{~mm}$ to facilitate the new FFC connectors and avoid all clearance issues associated with the increased mated height and the system stack. Two identical FFC 


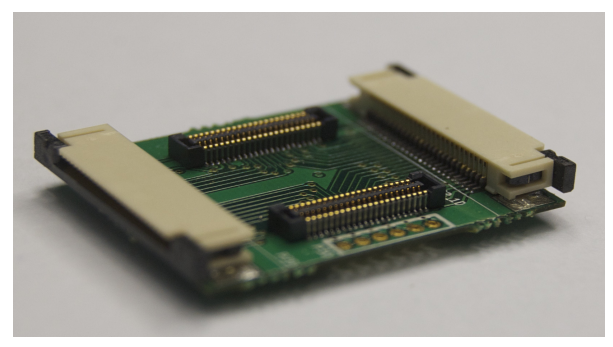

(a) Breakout Board

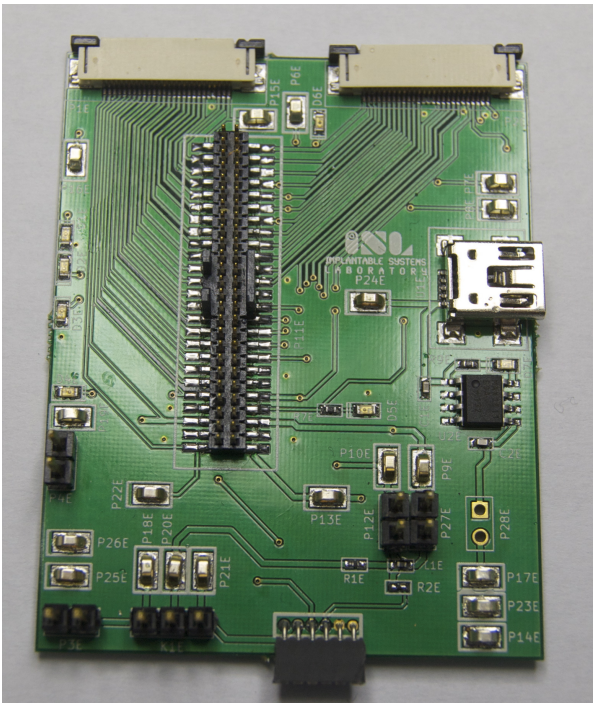

(b) Development Board

Figure 4.29: (a) Breakout board and (b) Development boards of the second physical prototype.

connectors are placed on the development board to transfer the signals from the vertical bus to the development board. Flat flex cables, $0.305 \mathrm{~m}$, Fig. 4.29, 4.30, are used for this connection. No additional components are placed on this PCB, only the two FFC connectors and the vertical connectors. The breakout board provides only signal routing to the development board and serves no other functional purpose inside the system stack.

\subsubsection{Development Board}

The second board in the testing platform is a much larger PCB that contains programming, charging and debugging capabilities. This PCB receives the communication bus signals from the FFC cables and distributes them to various sections of the boards, Fig. 4.29(b), These sections are separated into battery charging, power management, USB programming, and testing components.

\section{Battery Charging}

A mini USB connector is added to the PCB to provide a direct connection to USB power for the MAX1811 USB charging IC. Test pins are provided to test the input voltage from the USB 


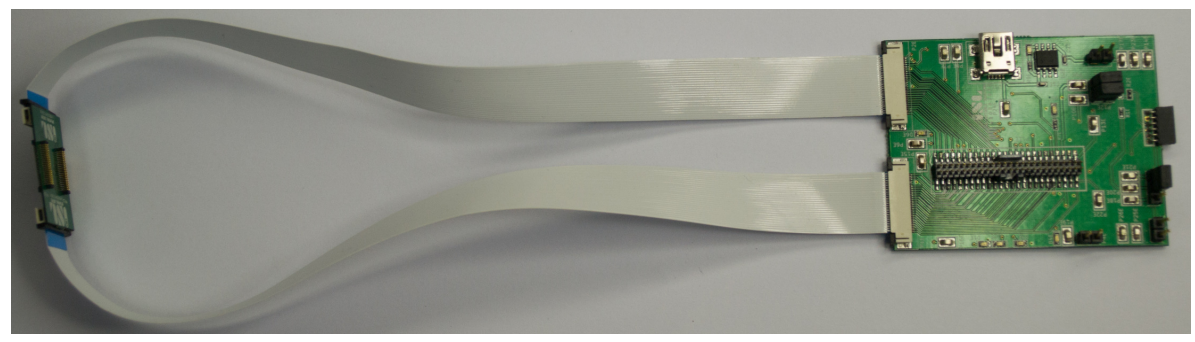

Figure 4.30: Development and Breakout Module connected via flex cables.

connector and the output voltage of USB charging IC. This power signal is routed directly to the FFC connectors and directly connects to the external battery to charge it. An LED is also used to indicate when the battery is being charged.

\section{Power Mangement - Development Board}

Power management and testing functionality is also designed into the development board. The main telemetry system can be powered through the attached battery or by the development board in testing. The power signals are routed to test points along the PCB to test for correct voltages for the system voltage, interface voltage and $1.8 \mathrm{~V}$ reference. Another design feature of this PCB is enabling an external power connection to the development board, which in turn can power the whole system without the use of a battery. This feature is extremely important in testing and debugging the system as precise current consumption can be calculated. All three main telemetry system voltages can be applied externally through custom 2 pin connectors located on the PCB. Additional connectors are added to switch between battery power and external power as to not damage the electrical components.

\section{Programming and Testing - Development Board}

A programming interface is provided on the development board to program the MSP430 from IAR Workbench The programming interface also provides external power to the system and must be disconnected from any additional power source when connected.

Finally, multiple debugging connections are added to the PCB. There are a total of six 
LEDs that are directly connected to the pins of the microcontroller and are controlled when testing. Furthermore, test pins are provided for all outputs of the interface module as well as various other inputs and outputs of the microcontroller. Lastly, all the signals from the FFC are also routed to a larger external breakout connector. This is 50 pin connector that is used for additional pin out or connection to an external board. The breakout and development boards provide all the testing functionality to the wireless telemetry system along with USB charging and programming features.

\subsection{Telemetry System Assembly Implementation}

The system prototype is designed with the primary aim to explore physical limitations of the state-of-the-art discrete components technology in respect to the minimum possible physical size, and the system's power consumption requirements. As a secondary goal, we designed the development RF telemetry platform based on a modular topology, which enables us: a) to explore and optimize the overall system performance; b) to verify the system's in-vivo performance with medium animal subjects (e.g., large rat or a rabbit); and c) to further reduce the system's size by replacing each module with a custom single die IC design. With this strategy, the current prototype will serve as the development platform, where the newly designed IC modules will be tested within the real system.

The telemetry system can be optimized for different structures (i.e., planar, flex-rigid PCB, cubic, etc.) for different animal types or for commercialization purposes where the goals may be different (design for manufacturability, disposable, etc.). These different structures allow specific attributes of the telemetry system to be optimized i.e., volume reduction only, antenna structure, implant location, etc. In this design, a cubic structure was chosen for the implant. This structure allows volume to be minimized by having each module stacked on top of each other and allows for a full modular topology.

The telemetry system is assembled vertically, creating a cubic structure using five separate 

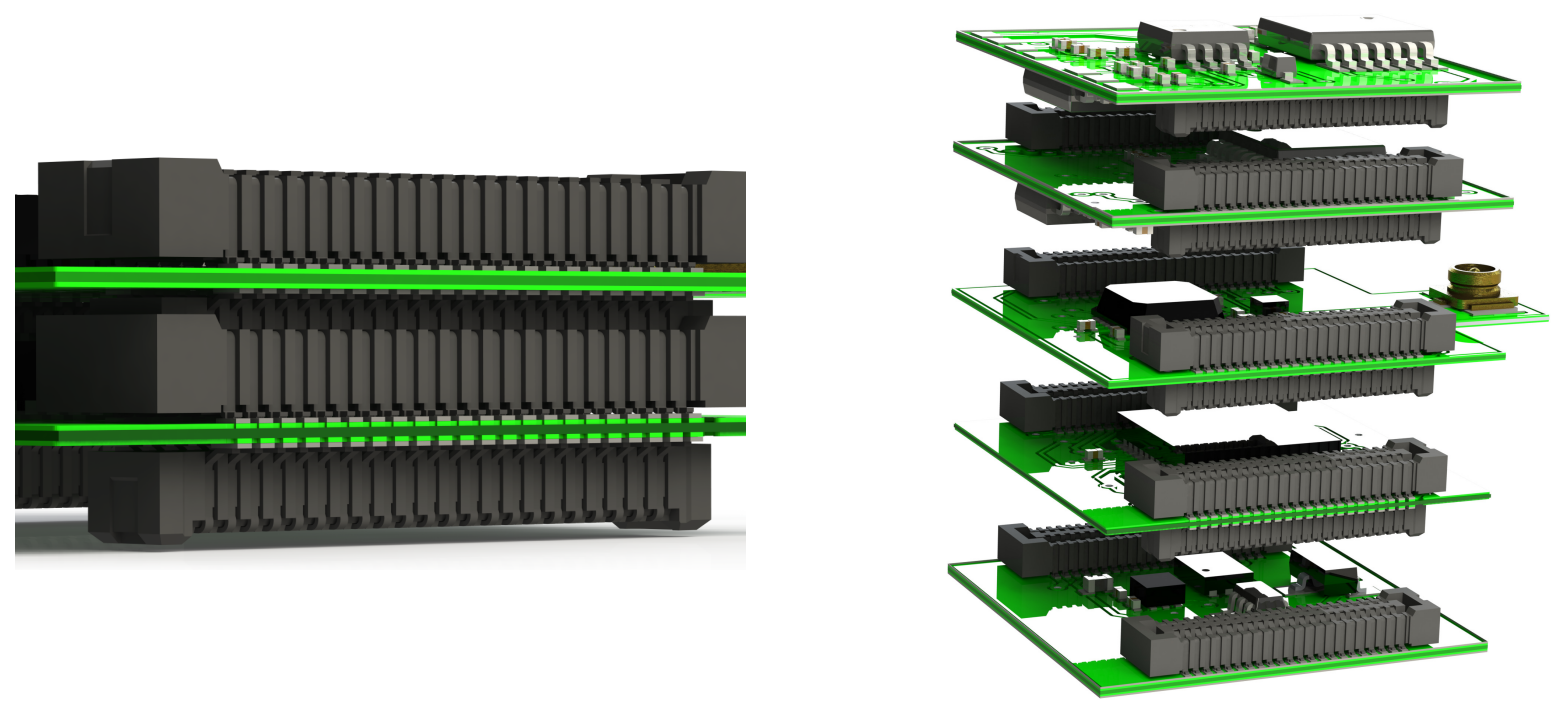

Figure 4.31: Close up 3D rendering of stacked vertical connectors (left), and exploded view of the telemetry system with 5 PCBs from the PV sensor interface at the top, to the power supply module at the bottom (right).

PCB's with a thickness of $0.4 \mathrm{~mm}$ and dual micro-sized connectors with a mated height of $1.5 \mathrm{~mm}$, Fig. 4.31. The stack up of the system from top to bottom is as follows: interface module board 1, interface board 2, RF module, microcontroller module, and power module. 3D modelling was completed on the prototype to ensure clearance and overall construction before the physical prototype was manufactured. The system including the battery is $(15 \times 15 \times 11) \mathrm{mm}^{3}$ (i.e., $2.475 \mathrm{~cm}^{3}$ ). During testing the breakout board is added to the stack to connect the communications bus to the development board. To ensure full clearance the breakout board must be connected directly above the power module to allow for clearance between the breakout and RF boards. The final assembled wireless telemetry system is shown in Figure 4.32, comparing it to the $3 \mathrm{D}$ rendering. 

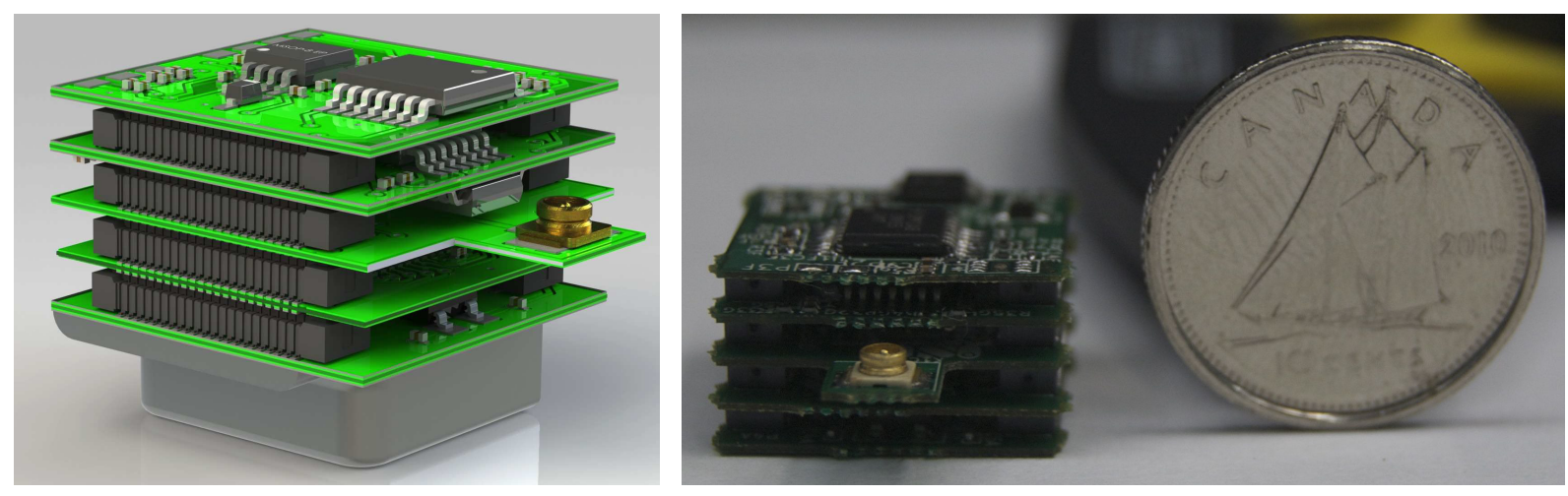

Figure 4.32: Full 3D rendering of assembled wireless telemetry system with battery (left), and photograph of fully assembled prototype (without battery) next to a Canadian dime (right).

\subsection{Control Architecture and Current Consumption}

The analog output signals of the PV sensor interface module: I, Q, and Pressure are processed by the microcontroller using a 10-bit SAR A/D converter. This implant is designed for medium sized hearts, with a peak heart rate of $\sim 240 \mathrm{bpm}$ or $4 \mathrm{~Hz}$. The highest nyquist tone of the heart beat is captured by a sample rate of $10 \mathrm{~ms}(100 \mathrm{~Hz})$. The sample rate is controlled by an internal timer clocked by an internal VLO set to trigger an interrupt every $10 \mathrm{~ms}$. The implant control architecture is separated into two main sections: 1) Main CPU operation and 2) Timer interrupt, Fig. 4.33. The CPU loop's main function is to either send a data packet to the external receiver or turn the transceiver into Rx mode from sleep. Various command flags are used to determine these operations, i.e., TX and RX. These flags are controlled by the control flow inside the timer interrupt.

\subsubsection{Implant Initialization}

The implant is initialized in three steps: a) board initialization; b) calibration of Low-Power Oscillator (VLO); and c) transceiver configuration. The implant initialization is completed in $27 \mathrm{~ms}$ with the corresponding instantaneous current profile shown in Fig. 4.34. First, the configuration of the ADC, clocks, counters, and GPIO are completed during board initialization. 


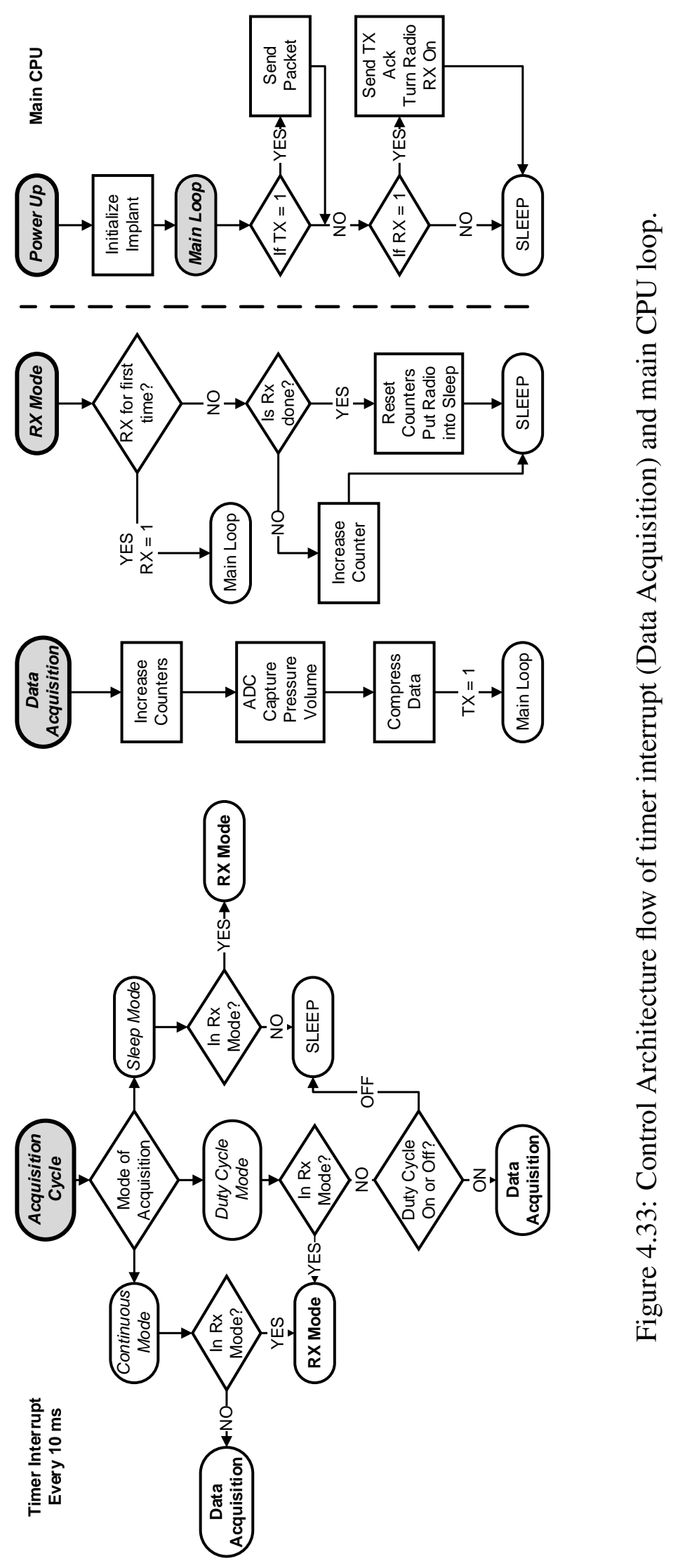




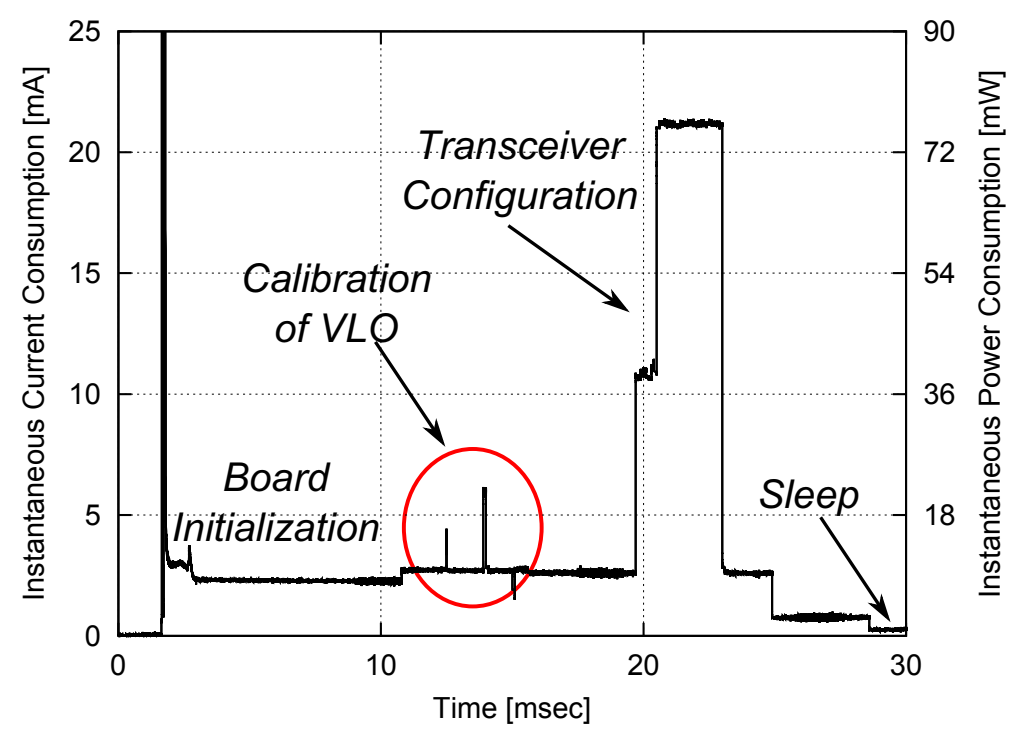

Figure 4.34: Implant initialization: Board Initialization, Calibration of VLO, and Transceiver Configuration.

The VLO must be calibrated to ensure the proper sample rate, which is achieved by calibrating the temperature and process dependent VLO to the more accurate factory calibrated main clock of the microcontoller. This method ensures that sample rate is $10 \mathrm{~ms}$ each time the implant is powered on. To ensure proper calibration and minimal drift over time, the same procedure may be repeated while the system is implanted. Lastly, the RF parameters of the transceiver are configured and the implant placed into sleep mode.

\subsubsection{Data Acquisition}

The implant is designed to operate in three distinct operational modes: 1) Continuous, 2) DutyCycle, and 3) Sleep cycle. These modes control the data acquisition and receive modes. Within the acquisition cycle, Fig. 4.33, data acquisition occurs in three stages. First, all appropriate counters are increased, followed by the sampling and conversion of the analog input signals (I, Q, Pressure) and lastly, the data is compressed. Rather than use three separate integer values (16 bits) to represent three $10-$ bit samples (48 bits), the data is merged into a single $32-$ bit value per acquisition, i.e., the payload for the system. In addition, a receive mode is used to 
accept instructions from the base station. Regardless of what acquisition mode the implant is in, the system always checks whether the device should enter receive mode. This is done through the use of two counters, $\mathrm{RX}_{\text {TimeofF }}$ and $\mathrm{RX}_{\text {Timeon }}$. These counters are independent from each other and can be set to any time frame, for example 20min off and $1 \mathrm{~min}$ on. Receive mode takes priority over data acquisition if they occur at the same time.

The average current consumption is reduced by using a digital I/O port on the microcontroller to disable the PV interface electronics after data acquisition, and by the microcontroller and transceiver entering sleep mode once a packet is sent. Furthermore, a memory buffer is used to store each payload before its transmission. The maximum size of this buffer is based on the available memory inside the microcontroller, in this MSP430, 856 bytes or 214 payloads. There is no reduction of average current consumption if the buffer size is 1 or 214 payloads, due to the increased transmission time required to send more payloads at once. However, the maximum buffer size is used in cases where the transmission window (i.e., receive window) is only available at a specific time rather than always listening. At a heart rate of $180 \mathrm{bpm}$, a fully loaded memory buffer contains approximately 8.5 PV loops, while the total acquisition time is $109 \mathrm{~ms}$ (not including processing and transmission). Additional on-board memory would increase the buffer size thus reducing the requirement of an continuous receive window.

When the memory buffer is full, the RF transceiver is turned on and the data payload is transmitted to the external unit, Fig. 4.33 Main CPU. The output power level is set to $-12 \mathrm{dBm}$ within the frequency bandwidth of $(2.4320-2.4356) \mathrm{GHz}$. The total time for one data acquisition cycle ( 1 payload) to complete is $4.45 \mathrm{~ms}, 508 \mu$ s for acquisition, $2.32 \mathrm{~ms}$ for processing, and $1.62 \mathrm{~ms}$ for transmission. The telemetry system is in a sleep state between measurement cycles, where the microcontroller and RF transceiver are placed in their respective low-power modes supplied by the main $3.6 \mathrm{~V}$ regulator. The VLO $(12 \mathrm{kHz})$ remains on to provide a timing base 
for the interrupt. Hence, the total current consumption in sleep (4.11) is $40 \mu \mathrm{A}$.

$$
\begin{aligned}
I_{\text {Sleep }} & =I_{\mu \mathrm{C} / \text { RF Sleep }}+I_{\text {Vreg Main }} \\
& =8 \mu \mathrm{A}+32 \mu \mathrm{A}=40 \mu \mathrm{A} .
\end{aligned}
$$

\section{Continuous}

In this mode, the PV interface electronics are powered on and sampled, followed by RF transmission. The instantaneous current consumption profile of the telemetry system in this mode with a $R_{\mathrm{L}}=100 \Omega$ and an output power of $-12 \mathrm{dBm}$, Fig. 4.35 , indicates that the telemetry system consumes $40 \mu \mathrm{A}$ in sleep, $3.46 \mathrm{~mA}$ sampling, $2.2 \mathrm{~mA}$ processing and $15 \mathrm{~mA}$ during $\mathrm{RF}$ transmission. This mode is repeated until $\mathrm{RX}_{\text {Time0FF }}$ is expired and at such time the $\mathrm{RF}$ transceiver is switched to receive mode for a predetermined amount of time, $\mathrm{RX}_{\text {Timeon }}$. Due to the transmit power level $(-12 \mathrm{dBm})$ and high receiver sensitivity $(-102 \mathrm{dBm})$, when the transceiver operates in the receive mode it has higher instantaneous current consumption of 20 $\mathrm{mA}$, which causes an increase in the average current.

\section{Duty Cycle}

In this mode, the implant operates similar to the continuous mode however, it is controlled by two counters Duty ${ }_{\text {on }}$ and Duty ${ }_{\mathrm{OFF}}$. These counters are configurable and set during implant initialization. For example, the duty cycle can be 6 min On and 54 min off or $10 \%$ duty cycle. The system enters sleep state once duty cycle window is over.

\section{Sleep Cycle}

In this mode, the implant is in sleep mode. The microcontroller and transceiver are powered down to reduce power consumption. In addition, the PV electronics are also shut-down. The system will stay in this mode indefinitely unless awaken via the RX mode. The instantaneous current consumption of the telemetry system in sleep cycle is $40 \mu \mathrm{A}$ in sleep and $20 \mathrm{~mA}$ when 


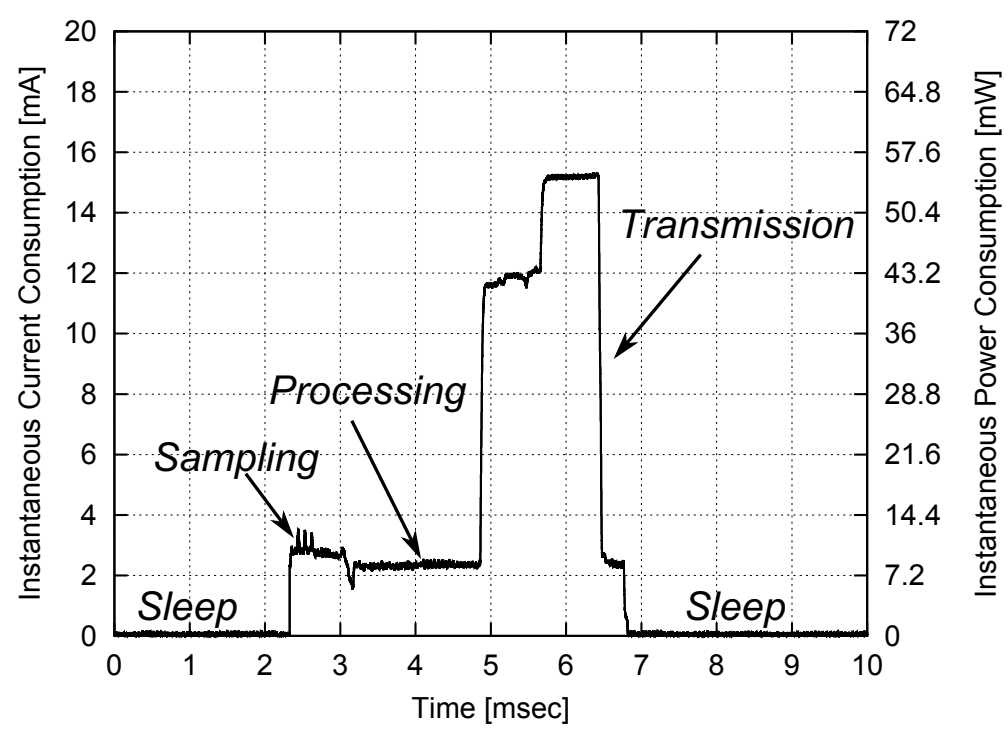

Figure 4.35: Instantaneous current and power consumption of the full telemetry system during a single data acquisition and transmission cycle through air with a load of $R_{\mathrm{L}}=100 \Omega$ and output power of $-12 \mathrm{dBm}$. Total time per acquisition: Sampling - 508 $\mu$ s, Processing - 2.32ms, Transmission - $1.62 \mathrm{~ms}$.

the transceiver is in RX mode. The RX on time will increase the average current consumption.

\subsubsection{Current Consumption}

The average current consumption along with the overall operational time of the telemetry system is found for each data acquisition mode separately, i.e., the implant would operate in one mode and continuously take measurements for the duration the battery life capacity, $40 \mathrm{mAh}$. For example, if the implant acquires data once every hour, i.e., 1 sample, to abide by the current profile in Fig. 4.35, the average current consumption is $40 \mu \mathrm{A}$ calculated by (4.12), where the instantaneous current measurement is separated into sampling $\left(\mathrm{I}_{\text {Interface }}\right.$ and $\left.\mathrm{I}_{\mathrm{Acq}}\right)$, processing ( $\left.\mathrm{I}_{\text {Process }}\right)$ and transmission $\left(\mathrm{I}_{\mathrm{TxA}}\right.$ and $\left.\mathrm{I}_{\mathrm{TxB}}\right)$.

As the telemetry system is only active for $4.45 \mathrm{~ms}$ per measurement and in this example per $\mathrm{hr}$, the dominant contribution to the average current is the sleep current. As indicated in (4.11), the main 3.6 V regulator's current consumption attributes the most to the sleep current hence, the most to the average current in a single measurement mode as well. Reducing the voltage 
regulator current consumption will scale down the average current of the telemetry system in this measurement mode.

$$
\begin{aligned}
I_{\text {Measurement }} & =I_{\text {Interface }} *(395.2 \mu \mathrm{s})+I_{\text {Acq }} *(112.8 \mu \mathrm{s}) \\
& +I_{\text {Process }} *(2.32 \mathrm{~ms})+I_{\text {TxA }} *(797 \mu \mathrm{s}) \\
& +I_{\text {TxB }} *(826.2 \mu \mathrm{s}) \\
& =(2.8 \mathrm{~mA}) *(395.2 \mu \mathrm{s})+(3.46 \mathrm{~mA}) *(112.8 \mu \mathrm{s}) \\
& +(2.2 \mathrm{~mA}) *(2.32 \mathrm{~ms})+(12 \mathrm{~mA}) *(797 \mu \mathrm{s}) \\
& +(15 \mathrm{~mA}) *(826.2 \mu \mathrm{s}) \\
& =28.56 \mu \mathrm{As} \\
I_{\text {Avg }} & =I_{\text {Measurement }} * \frac{\# \text { Meas }}{1 h r} * \frac{1 h r}{3600 \mathrm{~s}}+I_{\text {Sleep }} \\
& =\frac{28.56 \mu \mathrm{As}}{3600 s}+I_{\text {Sleep }} \\
& =40 \mu \mathrm{A}
\end{aligned}
$$

A summary of the average current consumption for different measurement cycles along with the total life cycle of the telemetry system with a $40 \mathrm{mAh}$ battery is shown in Table. 4.3 During continuous and duty cycle operation, the interface module is kept powered throughout the measurement cycle to ensure the proper startup conditions are met. It is observed that as the number of measurement cycles per hour is increased, the influence of the main voltage regulator's current consumption is reduced, placing more dominance on the instantaneous current consumption, mainly the Tx and Rx current consumption. There is no optimal cycle type/time and is to be optimized for the specific research protocol. For example, in some situations it is best to measure a $10 \mathrm{sec}$ window every $\mathrm{hr}$ where others it is best to measure $1 \mathrm{hr}$ continuously. 
Table 4.3: Average Current Consumption and Battery Life

\begin{tabular}{l|l|l|l}
\hline Cycle Type & $\mathbf{I}_{A v g}$ & Battery Life [hr] & Notes \\
\hline Continuous & $5.59 \mathrm{~mA}$ & 7.16 & $100 \mathrm{~Hz}$ sample rate \\
\hline & & & $1 \mathrm{Min}$ On \\
$59 \mathrm{Min}$ Off \\
Duty Cycle & $132 \mu \mathrm{A}$ & 302.07 & $\mathrm{No} \mathrm{Rx}$ \\
\hline & & & $\begin{array}{l}2 \mathrm{Min} \text { On } \\
2 \mathrm{Min} \text { Off } \\
\end{array}$ \\
Duty Cycle & $3.24 \mathrm{~mA}$ & 12.35 & $\mathrm{Min} \mathrm{Rx}$ \\
\hline Sleep & $373 \mu \mathrm{A}$ & 107.18 & $1 \mathrm{~min} / \mathrm{hr} \mathrm{Rx}$ \\
\hline
\end{tabular}

\subsection{External Receiving Unit}

An external receiving unit (Rx Unit) that consists of a microcontroller, wireless transceiver, and hardware UART board is designed to receive the transmitted data signals from the implanted system, Fig. 4.36, The data transmitted from the implant is received via the hardware wireless receive block and sent to UART module to be transmitted to a PC. The PC can be any desktop, laptop or other embedded device such as a Raspberry PI that is able to receive serial data. In this thesis, the PC is used is a Windows based system. The PC will then display the data received in either a terminal window or a custom based application like LabView. Each section of the External Receiving Unit will be discussed in the following subsections.

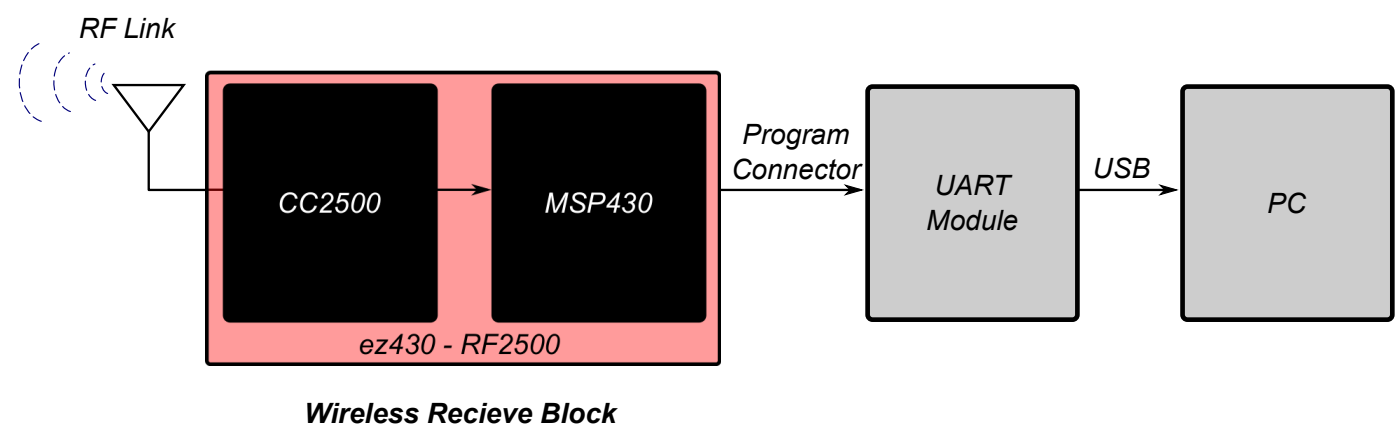

Figure 4.36: RX Unit Block Diagram that includes: the Wireless Receive Block, UART Module, and PC. 

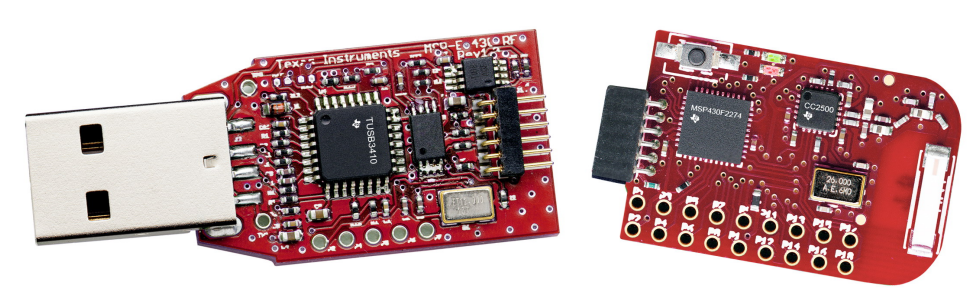

Figure 4.37: Wireless receive block including MSP430 programmer (right) and ez430-RF2500 (left) [29]. Copyright (CTexas Instruments.

\subsubsection{Wireless Receive Block}

The wireless receive block contains the same microcontroller, transceiver and PCB antenna as the implantable system, MSP430 and CC2500, Fig. 4.36. This block receives the incoming data from the implant, decodes packets and sends the data to the UART module. The MSP430 and CC2500 are configured similarly to their implantable system counterpart. The RF settings (carrier frequency, channel, etc) on the external device are identical to the implant to ensure a proper connection. The external CC2500 is configured to its highest sensitivity of $-108 \mathrm{dBm}$ for maximum range. The ez430-RF2500 is used as the external wireless receive block, Fig. 4.37 . A custom PCB can be created with external antennas and boost ICs to increase the range if required.

When data is received by the CC2500, an interrupt is triggered allowing the MSP430 to receive the data over the SPI bus and process it. As the incoming data is in the form of a 32-bit packet, it must be separated into each channel (I, Q, P) by using bit masking before it can be sent the PC. Each channel is a 10-bit ADC sample and is stored in a 16-bit integer. Although, each channel's binary data can be sent to the PC directly, it is more beneficial to convert each digit to ASCII characters for improved viewing on the PC. This has various benefits such as improved readability on the PC, no conversion required on PC software, and each digit can be sent in a single 8-bit UART transfer. In addition, by sending ASCII characters only, a simple text GUI can be created for the user. However, there two disadvantages to this method. First, additional space is required to store each channel. As described in Section 4.7.2, the maximum 
value for each channel is 1024 (decimal) or 3.6 V VDD. In binary, this is represented by a 10-bit value, stored in a 16-bit integer, however if each digit is converted to its corresponding ASCII character the size will be 4 bytes, or double the required storage per channel. For three channels (I, Q, P) and two additional characters for new line and carriage return, the total number of bytes to be sent to the PC per sample is 14 bytes. This leads to the second disadvantage, the increased BAUD rate required for MSP430 to PC transfer. Recall, in Section 4.7.2 that when each transmission occurs, a maximum of 214 samples are sent to the end unit. This data must be received by the external CC2500, processed by the MSP430 and sent to the PC and displayed before the next payload arrives. The minimum time required is when transmission occurs after each payload sample or $10 \mathrm{~ms}$, Fig. 4.35. As a result, the above steps must be completed in this time or data will be delayed within UART buffer on the PC. If the researcher is just logging data and not viewing it in real-time this is not much of a concern. However, when viewing data in real-time, the research may be required to act on the data received, for example change catheter location or record specific event. Thus, it is essential that the data be processed in a specific time frame.

The CC2500 and MSP430's processing are a fraction of the total time required in this process. The main bottleneck is the transfer speed of the serial connection to the PC also known as the BAUD rate. This is illustrated in the following calculations:

$$
\begin{aligned}
& \text { Sample per sec }=\frac{1}{10 \mathrm{~ms}} \\
& \text { Sample per sec }=100
\end{aligned}
$$

$$
\begin{aligned}
\text { Bytes per sec }(A S C I I) & =\text { Sample per sec } \times 14 \text { Bytes } \\
\text { Bytes per sec }(A S C I I) & =1400 \text { Bytes per sec } \\
B A U D(A S C I I) & =\text { Bytes per sec }(A S C I I) \times 8 \\
B A U D(A S C I I) & =11200 \text { BAUD }
\end{aligned}
$$




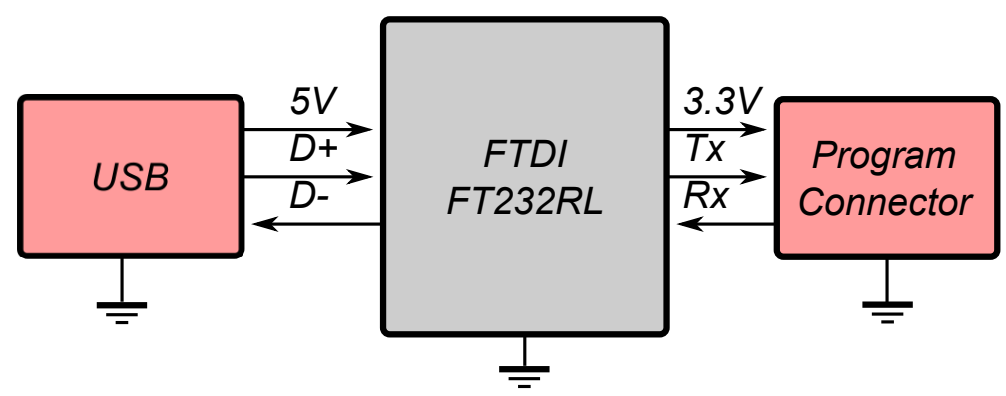

Figure 4.38: UART Hardware Module Block Diagram that includes the USB connector to send the PC data, FTDI IC, and Program connector to interface with the MSP430.

$$
\begin{aligned}
\text { Bytes per sec }(\text { Binary }) & =\text { Sample per sec } \times 6 \text { Bytes } \\
\text { Bytes per } \sec (\text { Binary }) & =600 \text { Bytes per sec } \\
B A U D(\text { Binary }) & =\text { Bytes per sec }(\text { Binary }) \times 8 \\
B A U D(\text { Binary }) & =4800 \text { BAUD }
\end{aligned}
$$

The required BAUD rate for ASCII transmission is 11200 and 4800 for Binary transmission. New line and carriage return characters are omitted from the binary transfer case. Typically, serial communication is done at 9600 BAUD, which can be emulated by software very easily and is the case for the standard MSP430 USB programmer. The programmer is included with the ez430-rf2500 and can be used to program any MSP430 using spy-by-wire. However, it's UART to PC transfer interface is not implemented by hardware, rather it is emulated by software, limiting the BAUD rate to 9600 . As illustrated by the above calculations, significant delay will occur if this BAUD rate is used. Therefore, a hardware UART module is required that is able to achieve a BAUD rate greater than 9600 .

\subsubsection{UART Module}

A custom made UART module is created with the following sub-blocks: USB, FTDI IC, and programming connector, Fig. 4.38.

The UART module receives the 1 byte UART packets from the external MSP430 via the programming connector. This connector is the same one used as on the development module. 


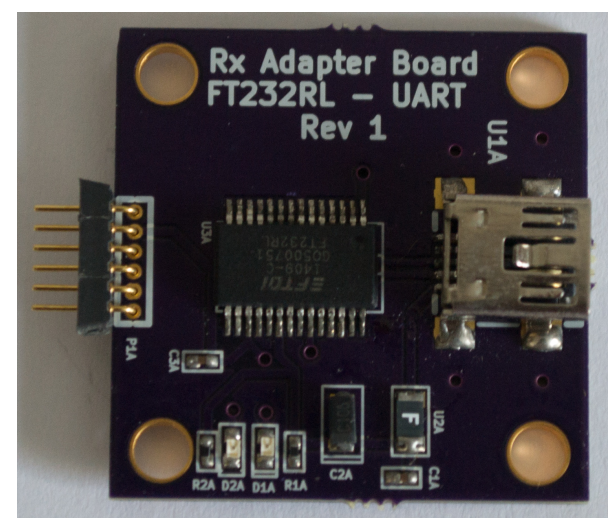

Figure 4.39: UART Hardware Module PCB.

The UART data lines are connected directly to the FTDI IC, FTDI FT232RL. The IC acts as an interface between the UART data lines of the MSP430 and the USB bus of a PC. The output pins of the FTDI FT232RL are connected to the data lines of a USB port, a mini USB jack is used. Power to the UART module, MSP430, CC2500 is provided via the $5 \mathrm{~V}$ USB voltage. The FTDI FT232RL contains a 3.3 V regulator used to power the MSP430 and CC2500.

A USB FTDI COM driver is required on the PC for proper operation of the module. These are readily available from the FTDI website, [256]. This driver enables the UART module to be viewed as a simple COM port on the PCB. Once installed, the FTDI IC is configured with the proper serial data transfer settings of 115200 BAUD rate. Additional settings can be set including hardware handshaking, buffering, etc. The UART module is manufactured on a 3 $\mathrm{cm} \times 3 \mathrm{~cm}$ two layer PCB, Fig. 4.39 . Holes are added to the design for mounting to a cage or external enclosure.

\subsubsection{PC / External Unit}

As the UART module acts as a simple COM port to the PC, the data can be read via any COM port reader such as Putty or a terminal (Mac OS X, Linux, etc). The incoming data is ASCII formatted as IIIIQQQQPPPP, where I, Q, and P represent the in phase (I) and out of phase (Q) signals from the volume measurement system and $\mathrm{P}$ representing the pressure output. There 


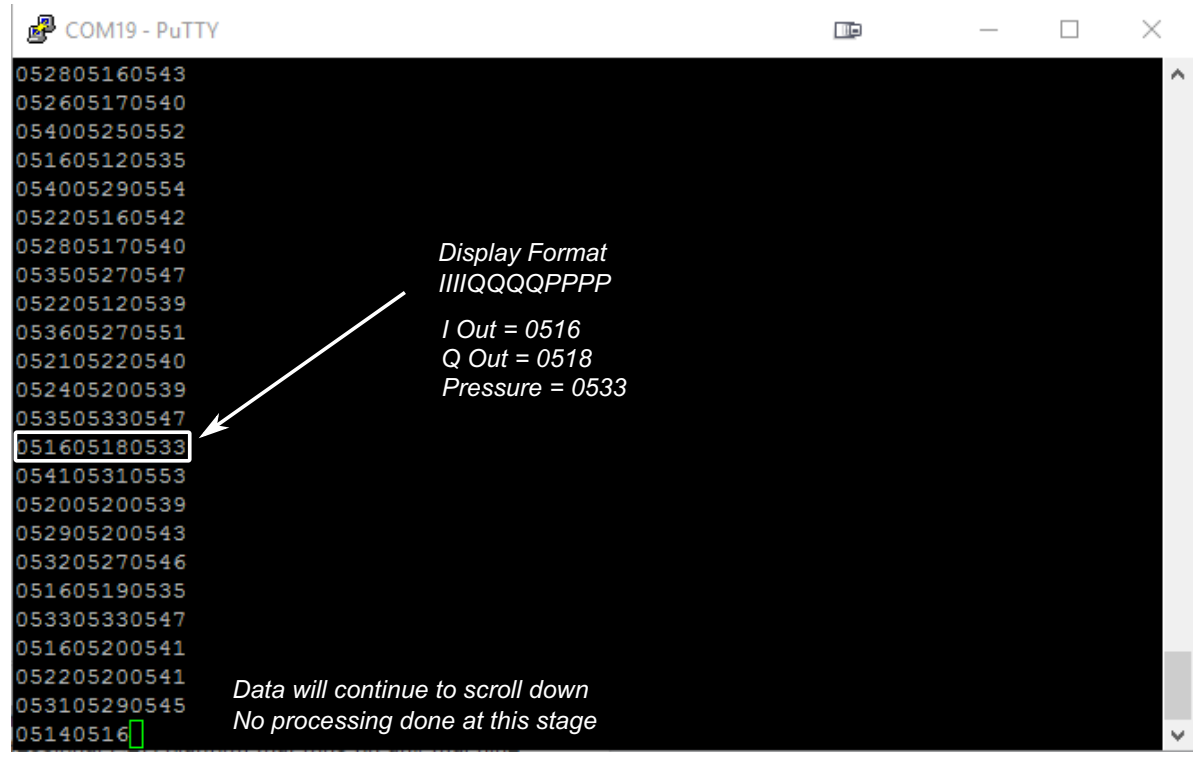

Figure 4.40: Raw Data Streamed to PC via Putty.

are are 4 characters per channel representing the maximum 1024 value. A total of 14 characters are sent with each sample, 12 data bytes and two new line characters. When viewing the data in a terminal window, each sample will appear as a single line with each new sample appearing below, Fig. 4.40. This viewing method is useful for testing the operation of the wireless link, data speed and $\mathrm{ADC}$ on the input side. However at this point, the incoming data to the terminal is not processed (raw voltages) and must be converted to admittance (magnitude and phase) and then to a volume. In addition the pressure must be converted to its calibrated value.

A custom application is created in LabView to further process the implant data. All three signals I, Q, and Pressure are saved and displayed numerically and graphically for the surgeon/medical technician to view, thus indicating catheter location during the implant procedure. In addition, the wireless receive unit is used to send instructions to the implant to change transmission power, acquisition mode, duty cycle and Rx duty times. A full in depth description of application and how its used within the in-vivo study is provided in Section 6.1. 


\subsection{Conclusion}

In this chapter, the design of the wireless implantable telemetry system is discussed. The system consists of four modules: power, signal processing, RF transmission, and analog front end. In addition, the communication bus and development module are discussed. Furthermore, details of the analog front end architecture are presented including an analysis into lock-in amplifiers and the pressure measurement system. Each module's physical implementation is presented culminating with the assembled telemetry system. The control architecture is presented indicating an average current consumption of $40 \mu \mathrm{A}$ and various measurement cycles are explored. Finally, the receiving unit is examined as well as how the data is displayed on the PC and further processed. 


\section{Chapter 5}

\section{In-Vitro Characterization *}

In this chapter, the in-vitro (non-implanted, external of body) characterization of the implantable telemetry system is presented. A phantom tissue study to model the effects of implant conditions and to determine the appropriate RF transmit power from the Pressure-Volume (PV) prototype [257] is described in Section 5.1] In Section 5.2 details of the implant capsule design for the PV system are presented, along with results from a 98 day water immersion study [258]. Calibration methodology is presented for both pressure and volume measurement circuits in Section 5.3. Lastly, dynamic PV system testing is discussed in Section 5.4 .

\subsection{Phantom Tissue Evaluation}

Extensive research has been documented outlying various tissue properties and standards [102, 147-150, 259-261]. It is well documented that the effect of tissue on a biomedical implant will impact its RF performance. Therefore, a phantom tissue study is undertaken to model the effects of implant conditions and to determine the appropriate RF transmit power from the PV prototype. A phantom tissue is a medium that mimics the electrical properties of real tissue. In some cases, researchers [262,263] have used meat prepared as food as a substitute for phantom

*Portions of this chapter are published in BIOCAS 2014 [257], Analog Integrated Circuits and Signal Processing journal [258], and Springer Biomedical Devices [240]. 
Table 5.1: Phantom Tissue Ingredients [32]

\begin{tabular}{l|c}
\hline Ingredients & Muscle [g] \\
\hline Distilled Water & 3375 \\
\hline Agar & 104.6 \\
\hline Sodium Chloride & 39.4 \\
\hline TX-151 & 84.4 \\
\hline Polyethylene Powder & 337.5 \\
\hline Boric Acid (Preservative) & 39.4 \\
\hline
\end{tabular}

tissues. This can provide a crude method of evaluating an antenna in an implantable setting however, it has several drawbacks including the lack of repeatability, and not knowing the process in how the meat has been prepared. Thus, a custom created chemical phantom tissue is created to ensure repeatability and accurate results. The outcome of this study is to determine the minimum RF output power that the transmitter $\left(\mathrm{T}_{x}\right)$ needs to provide to still receive data from the implant while having minimum power consumption in a typical medical measurement environment, i.e., instrument noise, non ideal antenna matching, spectrum interference, etc. Along, with RF output power, the maximum distance separating the implant and the $\mathrm{R}_{x}$ unit before data loss is also an important specification for a biomedical implanted system.

\subsubsection{Phantom Tissue Study Setup}

The tissue equivalent phantom is created from Agar, distilled water, Sodium Chloride, TX151, Polyethylene powder and Boric Acid (Preservative), Table 5.1 [32]. The agar allows the phantom to retain its shape and prevents water separation. The conductivity of the phantom is directly controlled with Sodium chloride and the relative permittivity is adjusted via Polyethylene powder. TX-151 is added to increase the viscosity of the medium and Boric Acid is used as a preservative. The mixing procedure is modified from [32] to suit the specific mixing environment and equipment available, this included the use of a microwave rather than gas burners.

The conductivity and relative permittivity of the phantom tissue are modelled to match that of muscle with a conductivity $\left(\sigma_{m}\right)$ of $2.21 \mathrm{~S} / \mathrm{m}$ and relative permittivity $\left(\epsilon_{r}\right)$ of 47 at 


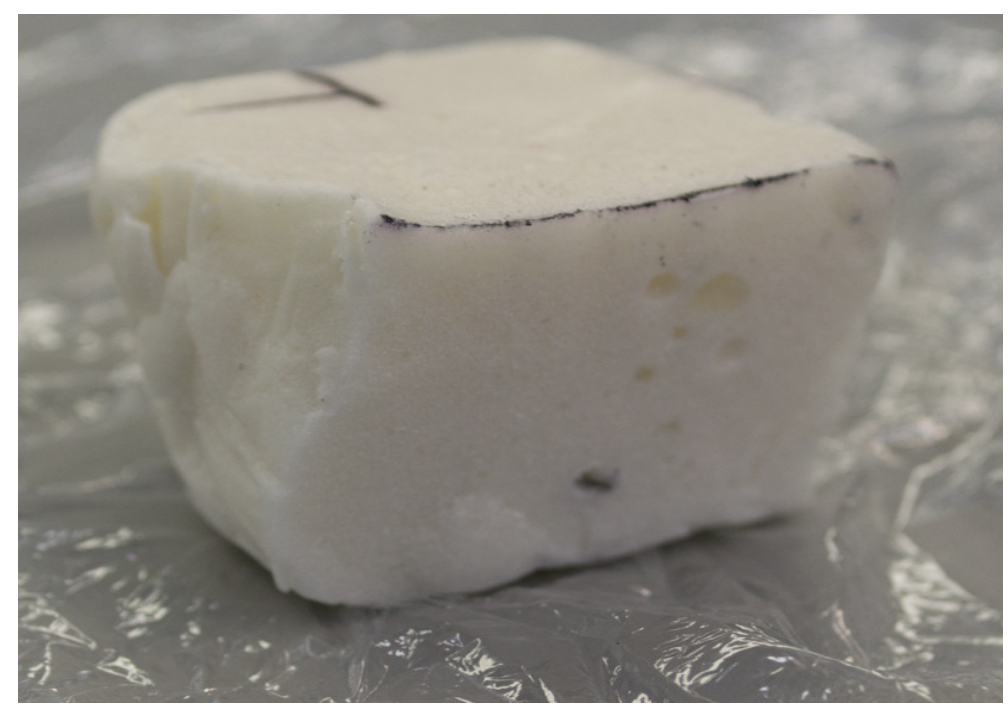

Figure 5.1: Phantom tissue used in study. $4 \mathrm{~cm} \times 4 \mathrm{~cm} \times 3 \mathrm{~cm}$.

$2.45 \mathrm{GHz}$ [264]. A full range of conductivity and relative permittivity of different biological mediums across a wide range of RF frequencies are available in the literature [147-149].

The phantom tissue is cut into a $4 \mathrm{~cm} \times 4 \mathrm{~cm} \times 3 \mathrm{~cm}$ structure, Fig. 5.1, where the antenna is inserted, as the typical antenna implant depth under the skin is in the range of $1 \mathrm{~cm}$ to $5 \mathrm{~cm}$ [259, 261], Fig. 5.2. The designed implant is expected to be placed at a similar depth underneath the skin in a in-vivo setting. This model emulates the tissue through which the transmitted RF signal must pass through while being transmitted from within the body. In a realistic implant setting the tissue will be made from a multi-layer structure comprised of skin, fat, muscle, bone, etc. To simplify our model, the tissue is modelled as muscle only.

An antenna attached to a spectrum analyzer is used as the $\mathrm{R}_{x}$ unit in this experiment and the PV prototype with and without phantom tissue is used as the $\mathrm{T}_{x}$ unit. Transmission of the PV implant is monitored with the distance range from $120 \mathrm{~cm}$ to $5 \mathrm{~cm}$ in steps of $20 \mathrm{~cm}$ between the $\mathrm{T}_{x}$ unit and the receiving antenna, with and without the phantom tissue inserted into the transmission path. The transmit power $\left(\mathrm{T}_{x} \mathrm{RF}\right.$ output power) is set to $0 \mathrm{dBm},-12 \mathrm{dBm}$, $-16 \mathrm{dBm}$, and $-20 \mathrm{dBm}$ to model a wide range of typical output power levels. An example test measurement setup is illustrated in Fig. 5.3 . 


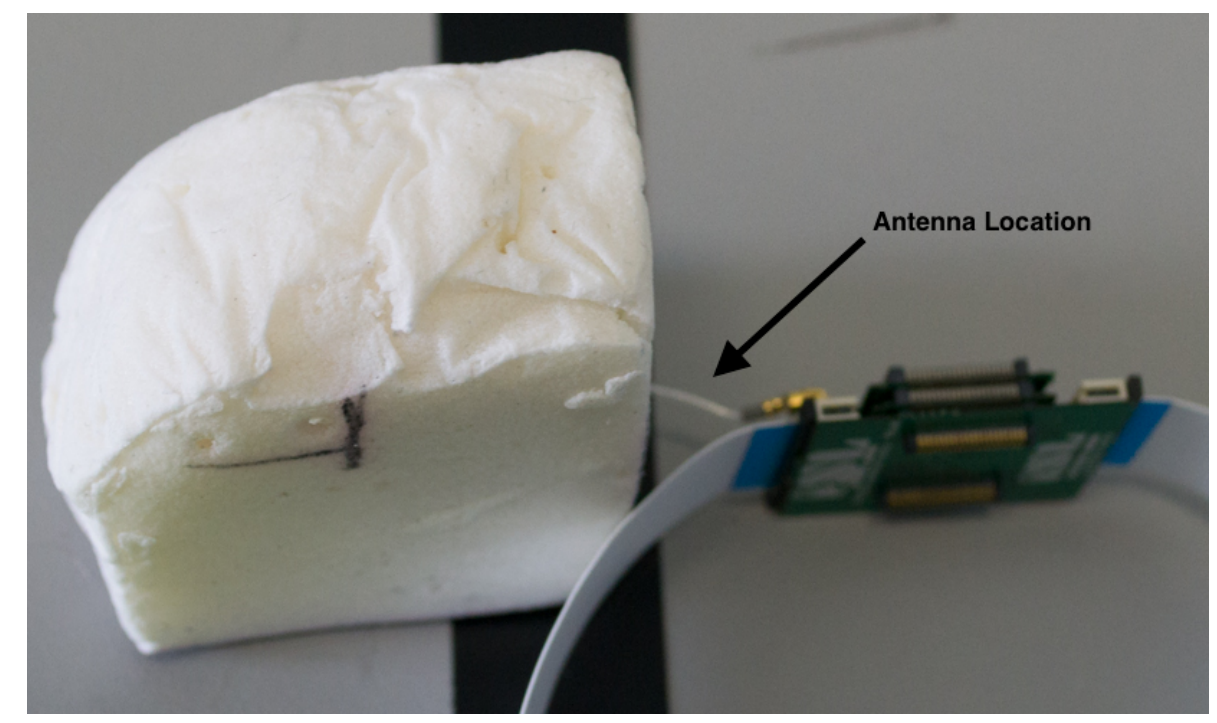

Figure 5.2: Implant antenna inserted into phantom tissue.

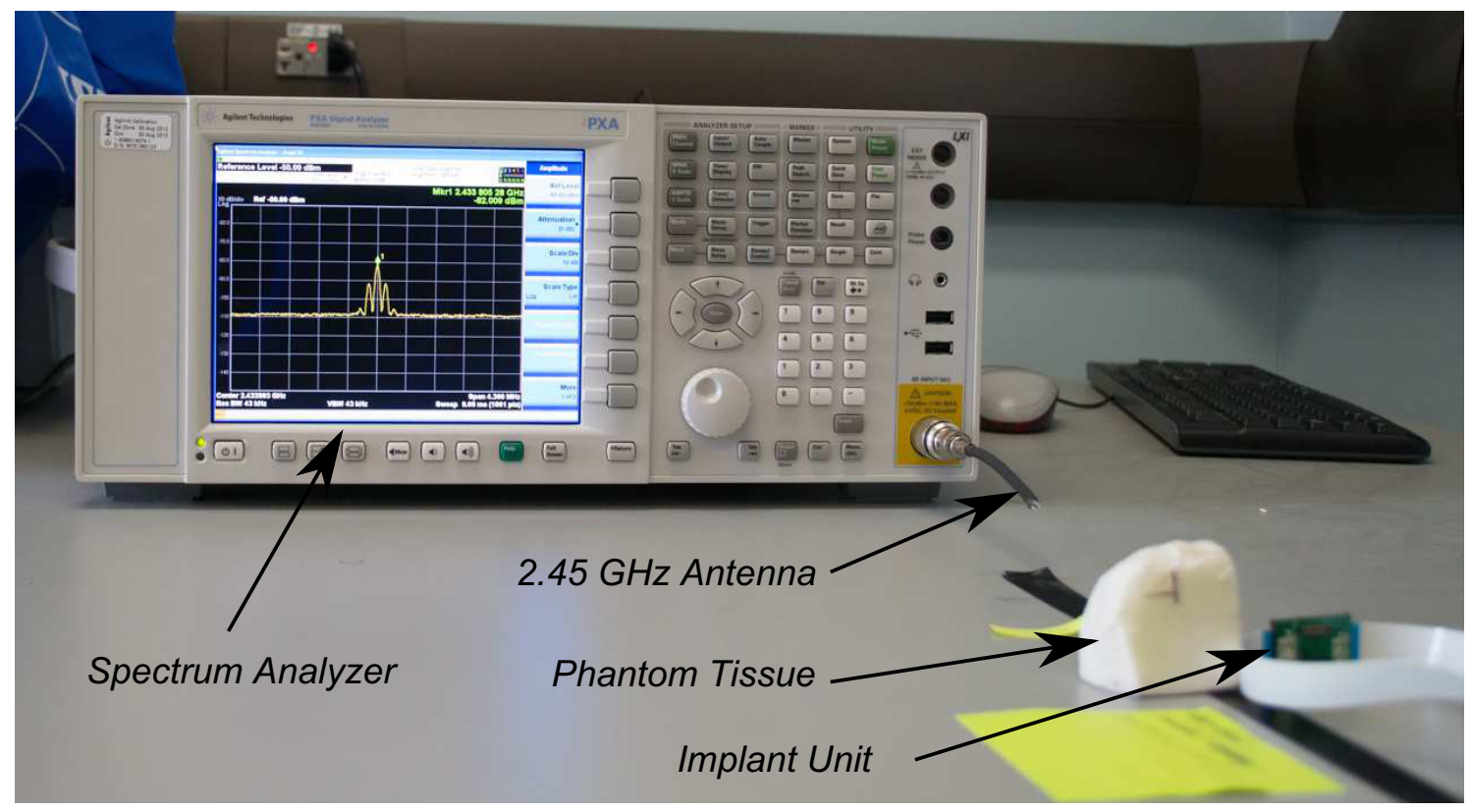

Figure 5.3: Experimental Measurement Environment used in study. Shown: Spectrum analyzer, $\mathrm{R}_{x}$ antenna, $\mathrm{PV}$ prototype with antenna inserted into $4 \mathrm{~cm}$ phantom tissue $10 \mathrm{~cm}$ away. 


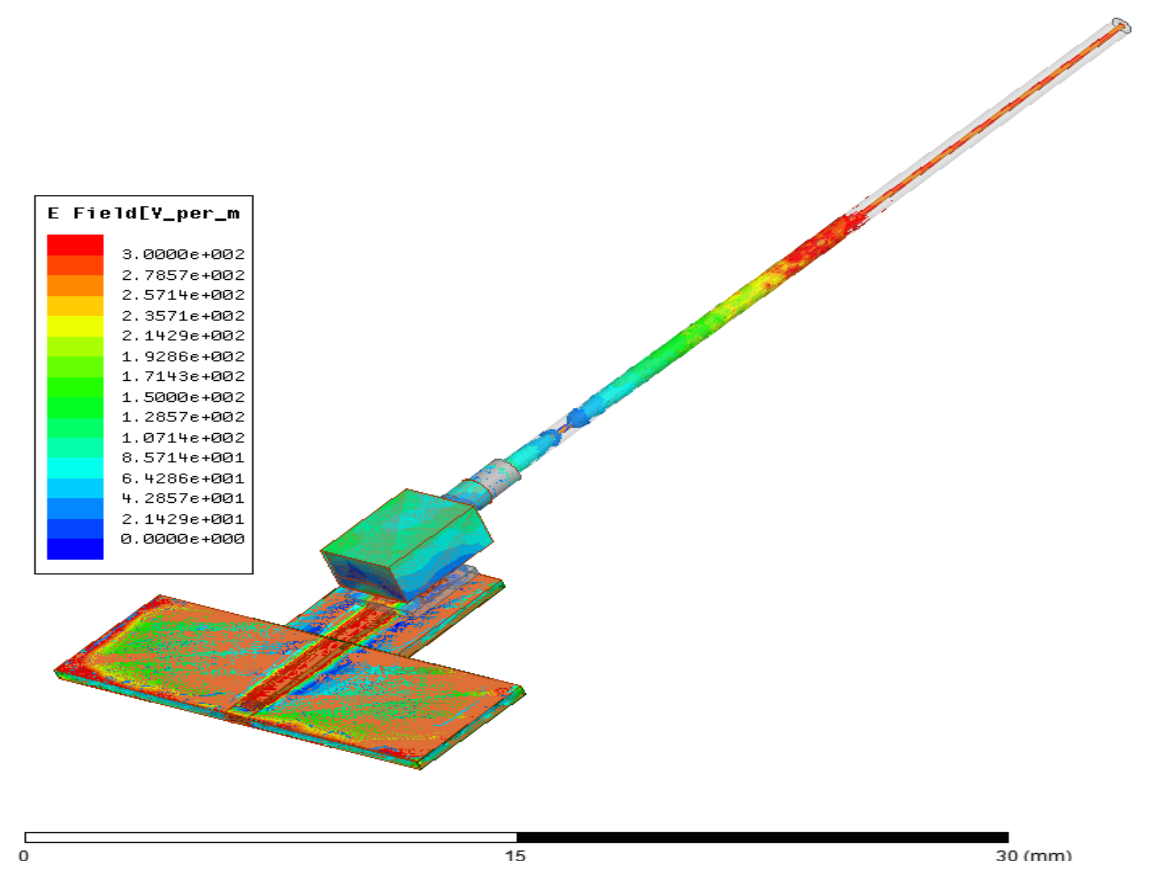

Figure 5.4: Designed 2.4 GHz monopole antenna used in EM simulations. Left: Electric Field Distribution with OdBm input power.

\subsubsection{EM Simulation Model}

A simplified EM model of the antenna and phantom tissue is created in HFSS to simulate the ideal measurement environment. In this model, the antenna on both the $\mathrm{T}_{x}$ and $\mathrm{R}_{x}$ side are matched to $50 \Omega$ and identical, the measurement environment is free of spectrum and measurement interference. The antenna implemented in the EM simulations is created from the physical and electrical parameters of the antenna used in the PV prototype. The antenna is a $30 \mathrm{~mm}$ monopole antenna that resonates at $2.442 \mathrm{GHz}$, Fig. 5.4. The VSWR and $\mathrm{S}_{11}$ of the modelled antenna are shown in Fig. 5.5 and 5.6, respectively.

The simulation model follows the measurement procedure outlined in Section 5.1.1 where the distance is swept from $120 \mathrm{~cm}$ to $5 \mathrm{~cm}$ at power output levels of $0 \mathrm{dBm},-12 \mathrm{dBm},-16 \mathrm{dBm}$, and $-20 \mathrm{dBm}$, with and without phantom tissue. The phantom tissue is modelled with the conductivity and permittivity at $2.45 \mathrm{GHz}$ representing the same electrical characteristics as realized in the experimental setup, $\sigma_{m}=2.21 \mathrm{~S} / \mathrm{m}$ and $\epsilon_{r}=47$. An example electric field 


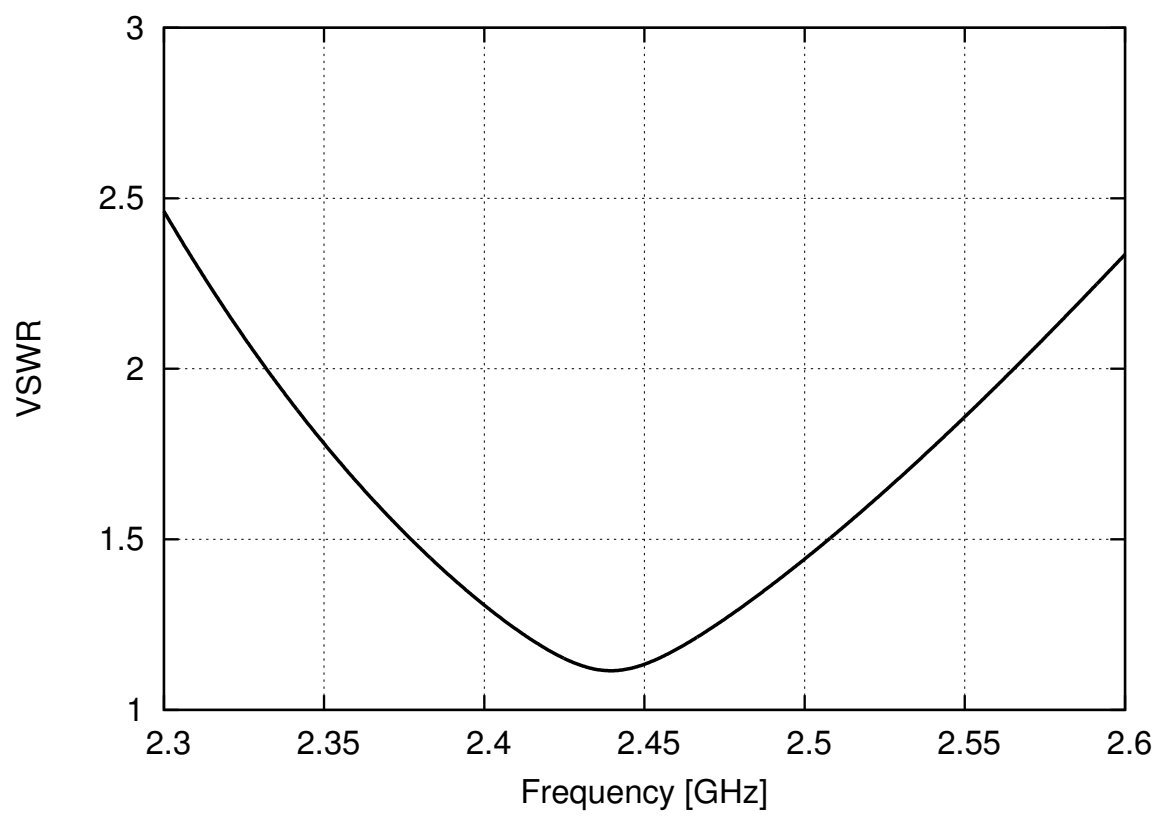

Figure 5.5: VSWR of 3D simulated antenna.

distribution of the $\mathrm{T}_{x}$ antenna is shown in Fig. 5.4, with an input power of $0 \mathrm{dBm}$. The corresponding $S_{21}$ plot of transfer link through phantom tissue at a distance of $20 \mathrm{~cm}$ is shown in Fig. 5.7 .

\subsubsection{Phantom Tissue Results}

Power loss without the phantom tissue $\left(P_{\text {loss }}\right)$, power loss with phantom tissue $\left(P_{\text {losst }}\right)$ and power absorbed by tissue $\left(P_{\mathrm{ab}}\right)$ are measured, Fig. 5.8 with the measurement setup outlined in Section 5.1.1. $P_{\text {loss }}$ represents the power losses due to the air transmission medium alone, $P_{\text {losst }}$ is the power losses due to the addition of phantom tissue with the air transmission medium and finally, $P_{\text {absorb }}$ is the power absorbed due to the phantom tissue alone (5.1), where $P_{\mathrm{r}}$ represents the power received without tissue and $P_{\mathrm{rt}}$ the power received with phantom tissue

$$
P_{\text {absorb }}=P_{\mathrm{r}}-P_{\mathrm{rt}}
$$

As the transmit power decreases $\left(T_{x}\right)$ and the distance from $R_{x}$ increases, the power re- 


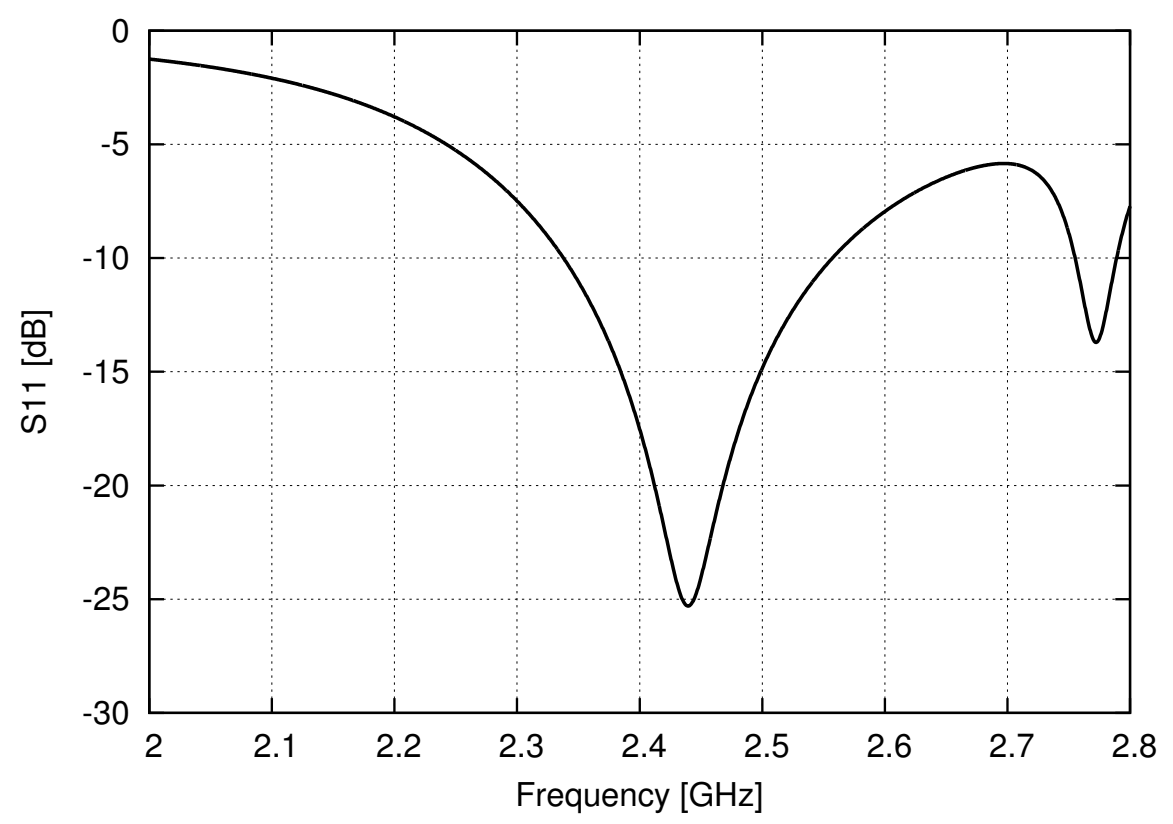

Figure 5.6: $\mathrm{S}_{11}$ of $3 \mathrm{D}$ simulated monopole $\mathrm{T}_{x} \mathrm{R}_{x}$ antenna.

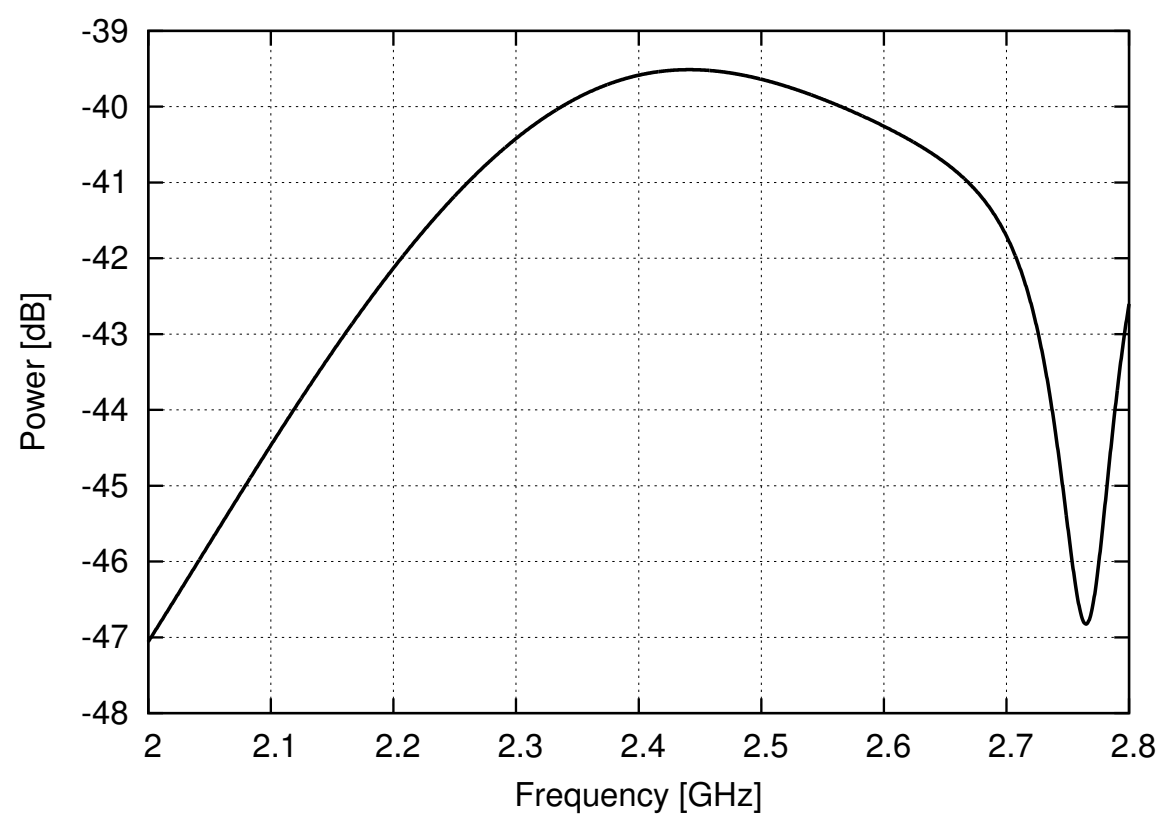

Figure 5.7: $\mathrm{S}_{21}$ of 3D simulated phantom tissue environment at a distance of $20 \mathrm{~cm}$ with $\mathrm{T}_{x}$ antenna inserted into phantom tissue model. 


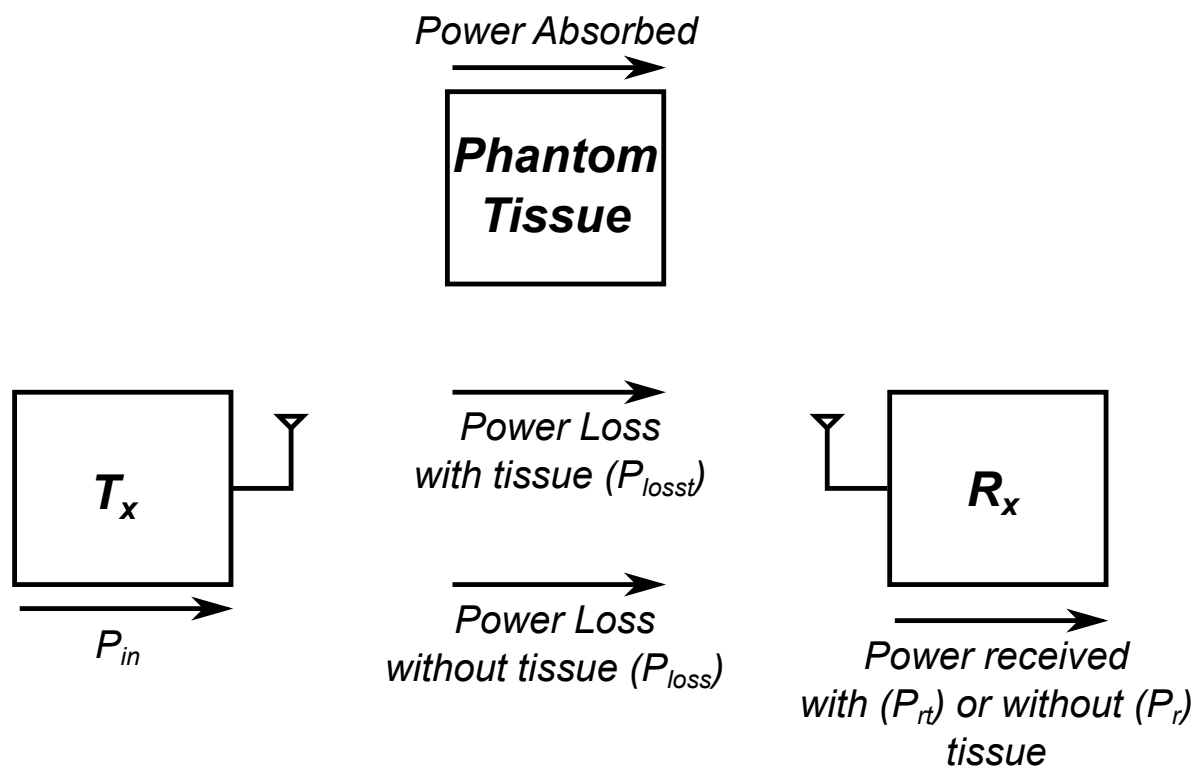

Figure 5.8: Calculation of absorption losses of phantom tissue.

ceived $\left(R_{x}\right)$ decreases, Fig 5.9 The received power approaches the sensitivity of the CC2500, $-104 \mathrm{dBm}$, when the transmit $T_{x}$ power is set to $-20 \mathrm{dBm}$. Although, the $R_{x}$ sensitivity threshold of the power is not reached while transmitting without phantom tissue, with the phantom tissue in the transmission path, at $-20 \mathrm{dBm} R_{x}$ power, this sensitivity limit is approached, i.e. the bit-error rate of the transmission becomes easily visible.

For an output $T_{x}$ power of $-12 \mathrm{dBm}$ the measured power losses along with simulated phantom tissue absorption are shown, Fig. 5.10 The power loss due to the air transmission medium, Fig. 5.10 (d), is significantly larger than the power absorbed by the phantom tissue, Fig. 5.10 (g). This indicates that the majority of the power loss from $T_{x}$ to $R_{x}$ is due to the air transmission medium. However, the loss due to the phantom is not negligible and is an average of $8.39 \mathrm{dBm}$ across the used distance range, hence the power absorption loss has to be accounted. In comparison, the simulated phantom tissue absorption loss is on average $8 \mathrm{~dB}$ higher than the experimental tissue absorption loss. This difference may be attributed to the non-ideal phantom tissue model mixture, the simplification of the simulation model, and the variation of $\sigma_{m}$ and $\epsilon_{r}$ of the simulation compared to the experimental phantom.

In addition, the power consumption of the transmitter is measured for output power levels 


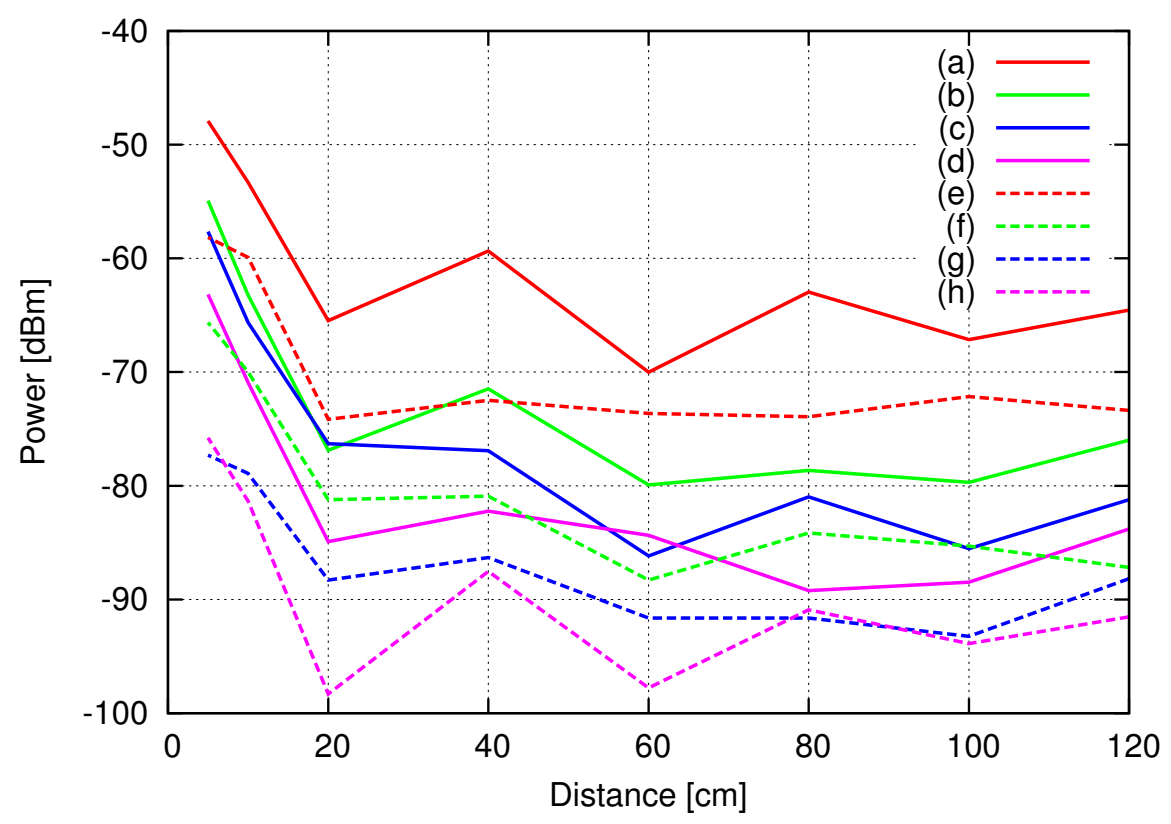

Figure 5.9: Comparison of different RF output power levels with and without the phantom tissue inserted. Without phantom tissue: (a) $0 \mathrm{dBm},(\mathrm{b})-12 \mathrm{dBm}$, (c) $-16 \mathrm{dBm}$, (d) $-20 \mathrm{dBm}$; With Phantom Tissue: (e) 0dBm, (f) $-12 \mathrm{dBm},(\mathrm{g})-16 \mathrm{dBm},(\mathrm{h})-20 \mathrm{dBm}$.

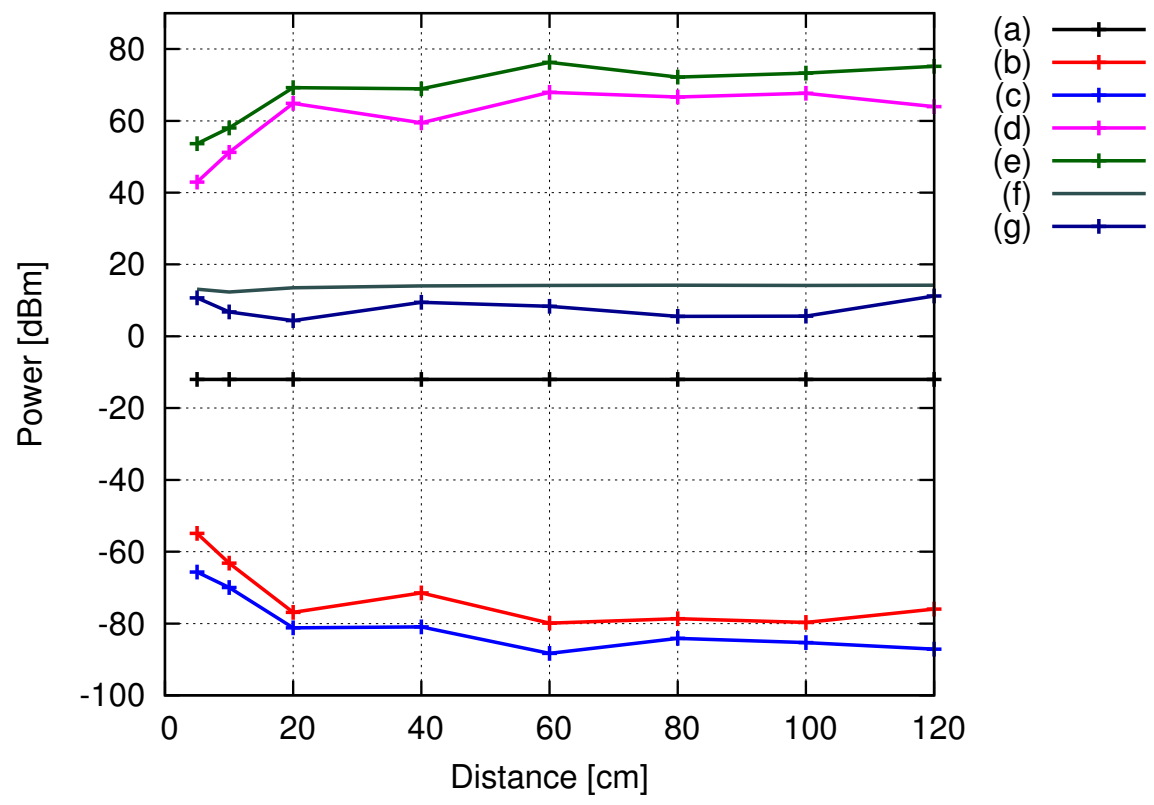

Figure 5.10: Receive power with and without phantom tissue for output $\mathrm{T}_{x}$ power level of $-12 \mathrm{dBm}$. (a) $P_{\text {in }}-$ Incident Power, (b) $P_{r}$ - Power received without tissue, (c) $P_{\text {rt }}-$ Power received with phantom tissue, (d) $P_{\text {loss }}-$ Power loss without phantom tissue, (e) $P_{\text {losst }}-$ Power loss with phantom tissue, (f) $P_{\text {absorb-sim }}-$ Power absorbed by tissue simulation, $(\mathrm{g}) P_{\text {absorb-exp }}$ - Power absorbed by tissue experimental. 
Table 5.2: Power consumption of Transmitter (CC2500) at various $T_{x}$ output powers, VDD $=3.6 \mathrm{~V}$

\begin{tabular}{c|c}
\hline Output Power $[\mathrm{dBm}]$ & Power Consumption $[\mathrm{mW}]$ \\
\hline 0 & 80.64 \\
\hline-12 & 43.92 \\
\hline-16 & 42.48 \\
\hline-20 & 39.96 \\
\hline
\end{tabular}

of $0 \mathrm{dBm},-12 \mathrm{dBm},-16 \mathrm{dBm}$, and $-20 \mathrm{dBm}$, Table 5.2 . There is a $2.52 \mathrm{~mW}$ difference between -16 and $-20 \mathrm{dBm} T_{x}$ output power and a $1.44 \mathrm{~mW}$ difference between -12 and $-16 \mathrm{dBm} T_{x}$ output power. The optimum output transmit power is a function of power consumption of the transmitter, receive power and distance of PV implant. A conservative choice is made for the output transmission power, $-12 \mathrm{dBm}$, which allows the implant to achieve full data transfer with and without phantom tissue at a distance of $120 \mathrm{~cm}$, well within the $-104 \mathrm{dBm}$ $\mathrm{R}_{x}$ sensitivity range of the CC2500. This power level choice has lower power consumption in comparison to $0 \mathrm{dBm}$. In comparison, the output power levels of -16 and $-20 \mathrm{dBm}$ offer lower power consumption at the cost of a decrease in the received power and higher risk of possible data loss at distances further away from the receive unit.

The phantom tissue study was completed for RF module with the external antenna only due to the complexities of placing the PCB antenna and the complete implant inside of a phantom while still operating the implant successfully. Although, the absolute results would be different as the antenna shape and size are different, the operating frequency is still the same and similar tissue absorptions would occur. Similar power output levels would be required when the PCB antenna is used. Further discussion on the differences between the external and PCB antenna is presented in the following Section 5.2 .

The outcome of this study helped to determine the minimum RF output power that the transmitter $\left(\mathrm{T}_{x}\right)$ needs to provide to still receive data from the implant while having minimum power consumption in a typical medical measurement environment. To achieve an optimized system, a specifically designed implantable antenna should be used, subject to future work. 
This work was published in the Journal Analog Integrated Circuits and Signal Processing, Springer under the title "In-Vitro RF Characterization of Implantable Telemetry System" in $2014[257]$.

\subsection{Implant Package Design and Evaluation}

In this section, the design and experimental details of a custom 3D bio-compatible implantable capsule that is used to house the wireless telemetry system designed in Chapter 4 is presented [30, 31]. The capsule is designed specifically for the dimensions and shape of the PV system and is 3D printed in four different materials: ABS, RDG720 Transparent Acrylic, ABS-M30i, and 316L Stainless Steel. Results of a 98 day long-term water bath immersion study and a 1 week short term study are shown to validate the design and encapsulation process. The aim is to design a minimum volume system that includes the electronic, the package, and the antenna.

In this work, although four different materials are evaluated, with the focus on the package version made of bio-compatible ABS-30i and sealed with silicone to prevent leakage and contamination; this package version will be used in the in-vivo experiment.

The rest of this section is organized as follows. In Section 5.2.1 the design of the 3D implant capsule are presented; in Section 5.2.2 details of the implant capsule preparation and immersion study results are described.

\subsubsection{Biomedical Implant Capsule Design}

Previous research has indicated various ways to protect the implanted electronics. Two mainstream methods found in the literature are: a) design of a customized capsule to house the internal electronics, and b) creation of direct silicone epoxy barrier on the PCB by using silicone dip method, [21, 88, 158]. In order to reduce the amount of risk for in-vivo applications and potential damage to both the system and the host subject a customized 3D capsule is designed in SolidWorks. The designs were manufactured using two 3D printers, one for plastic 


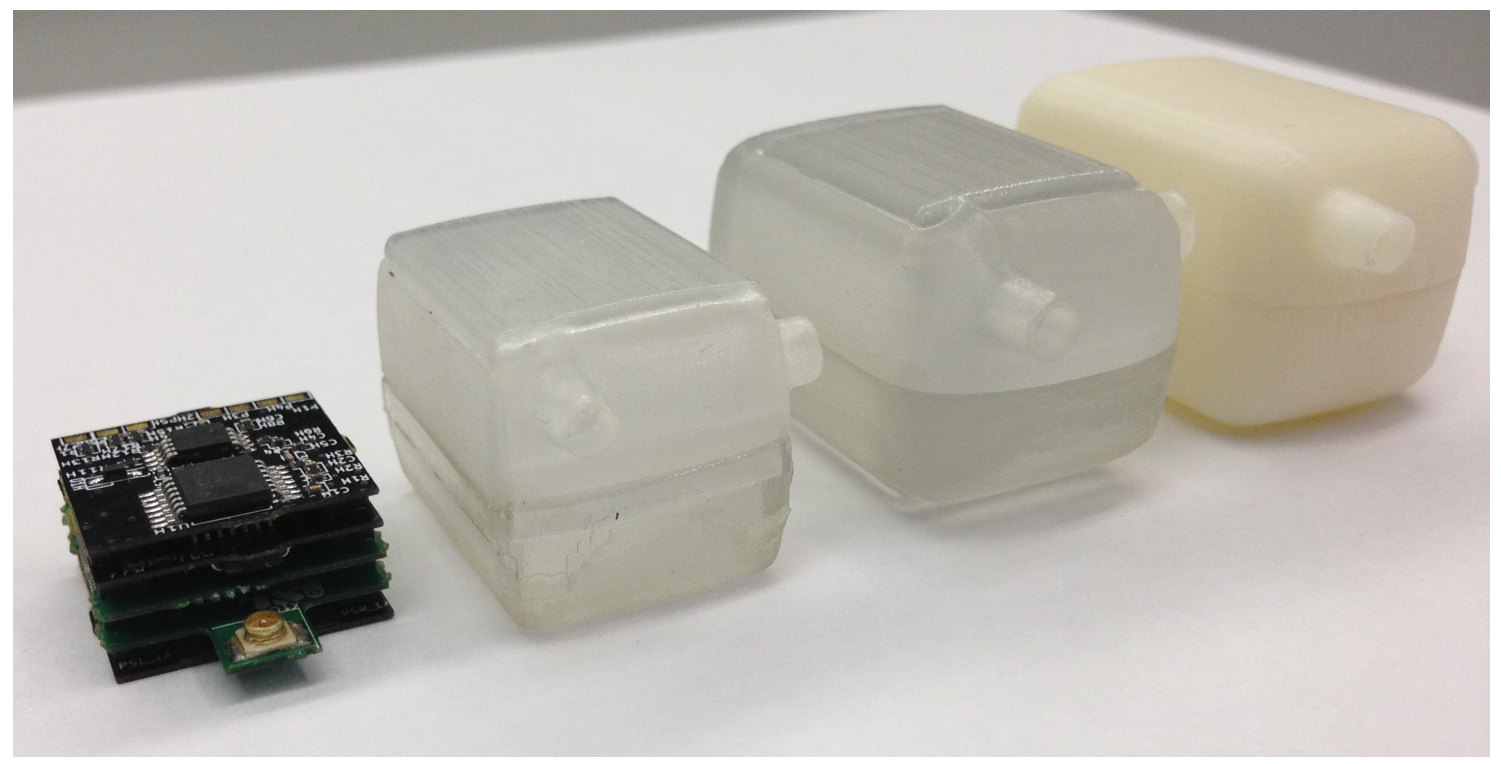

Figure 5.11: 3D Printed Plastic Packages for Pressure-Volume Implant from left to right: PV Telemetry system [30, 31], thin wall acrylic, thick wall acrylic, thick wall ABS-M30i (ABS not shown).

materials and one for metallic materials. Although larger in size than the direct silicon dip method, the capsule method results in much smoother edges of the overall system, thus less risk for possible fractures in the silicone coating in comparison to the sharp corners of the coated PCB system. In addition, the implant capsule allows for the electronics to be removed after in-vivo tests, thus prolonging the life-cycle of each implant.

The implant capsules are designed to accommodate the discrete PV system outlined in Chapter $4[30,31]$ and they consist of a front port hole for catheter access, a side antenna access hole, and is split into top and bottom halves, where the battery and the implant system are placed in the bottom half. Starting from a custom SolidWorks model, four plastic packages and one metal package are 3D printed using four different materials: 316L stainless steel, ABS, RDG720 Transparent Acrylic, and ABS-M30i, Fig.5.11, Fig.5.12(ABS not shown).

The first experimental package was made from acrylic with very thin side walls, thus only slightly larger than the prototype itself, Fig. 5.14(left). However, the reduced size of the acrylic walls, whose thickness was designed to be $0.79 \mathrm{~mm}$, poses a great challenge as the walls are difficult to form correctly. The outside package dimensions are designed to approximately 


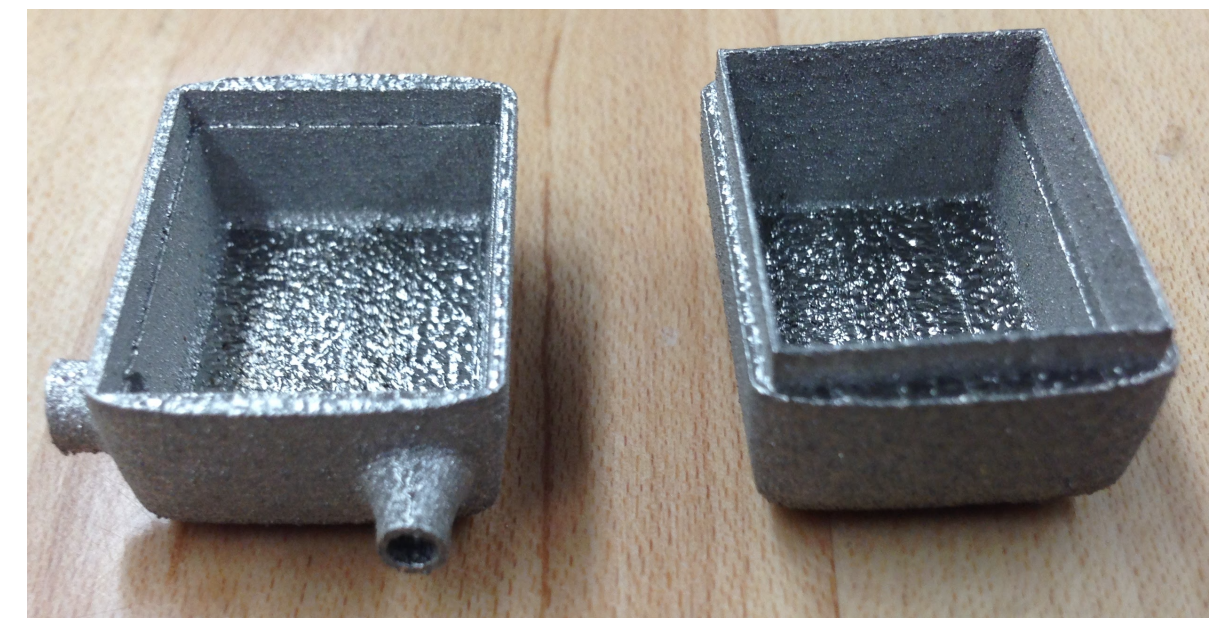

Figure 5.12: 3D Printed metal packages for pressure-volume implant: thin wall 316L stainless steel top (left) and bottom (right).

$24 \mathrm{~mm}$ by $18 \mathrm{~mm}$. In addition, the tolerances of the port holes for the antenna and catheter are difficult to control with this material at this scale. This is illustrated when two identical copies of the top half are printed, Fig. 5.13. Both parts have the same size and shape, however, the capsule on the left has issues with the wall thickness and the version on the right has errors in both the port hole and the smoothness of the side walls. Instead, the thin walled version of the package is printed using stainless steel, which does not have the manufacturing and repeatability issues that the thin walled acrylic version has. The Stainless Steel thin version is then used in this experimental evaluation.

A second, slightly larger, version of the package is created in ABS, RDG720 Transparent Acrylic, and ABS-M30i, Fig. 5.11. That is, the size tolerances are increased as well as the side wall thickness (designed to be $1.26 \mathrm{~mm}$ ), to prevent cracks from forming and to increase its overall robustness, Fig. 5.14(right).

The three plastic materials are selected based on their compatibility with the ISO 10993-6 bio-compatibility standard (ABS-M30i); availability from a wide range of 3D printers (ABS); and the transparent nature of the material, thus enabling the internal cavity to be directly monitored throughout the study (RDG720 Acrylic). Although, heavier than the plastic materials, 316L Stainless Steel is selected to evaluate the 3D metallic printer's ability to create the small 


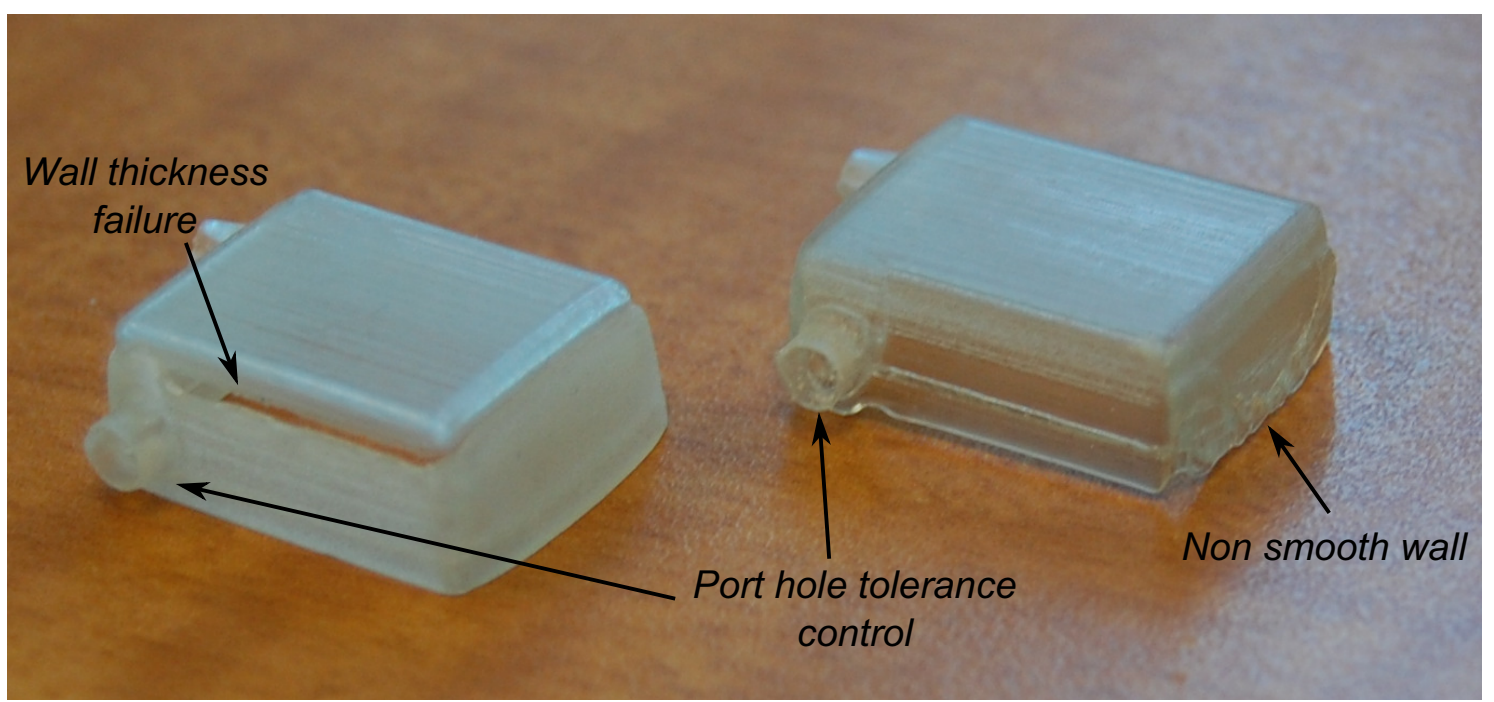

Figure 5.13: Examples of errors when 3D printing the thin wall version in plastic.
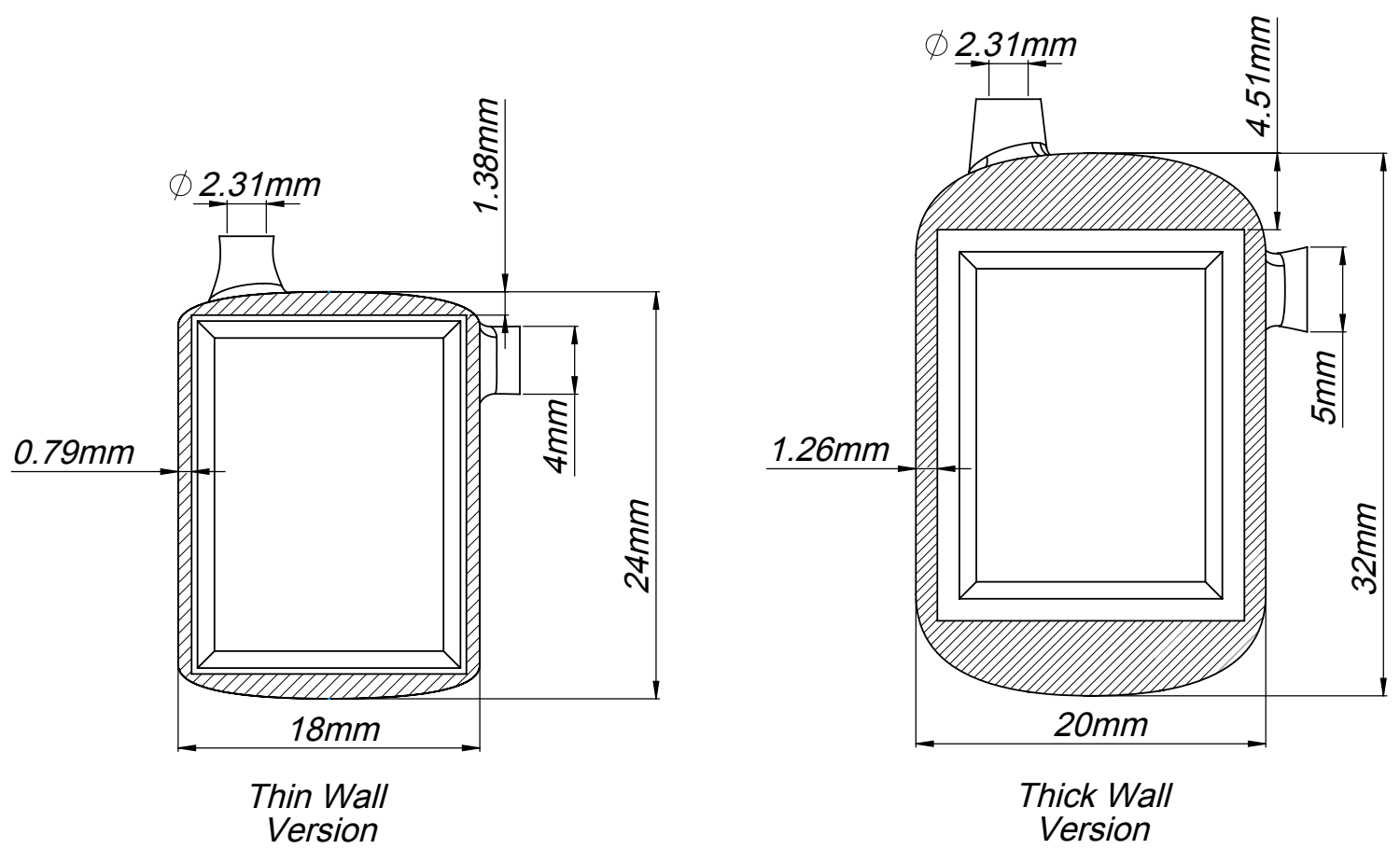

Figure 5.14: Implant package cross-section view: Thin wall package with $24 \mathrm{~mm}$ by $18 \mathrm{~mm}$ outer dimensions (left); and thick wall package with $32 \mathrm{~mm}$ by $20 \mathrm{~mm}$ outer dimensions (right). Outer wall thickness noted by hatched lines. 
Table 5.3: Summary of 3D capsules printed

\begin{tabular}{|c|c|c|}
\hline & Thin Version & Thick Version \\
\hline Wall Thickness & $0.79 \mathrm{~mm}$ & $1.26 \mathrm{~mm}$ \\
\hline Outer Dimensions & $24 \mathrm{~mm} \times 18 \mathrm{~mm}$ x $18 \mathrm{~mm}$ & $32 \mathrm{~mm}$ x $20 \mathrm{~mm} \times 20 \mathrm{~mm}$ \\
\hline Materials Printed & $\begin{array}{l}\text { RDG } 720 \text { Transparent Acrylic Material } \\
\text { 316L Stainless Steel }\end{array}$ & $\begin{array}{l}\text { RDG } 720 \text { Transparent Acrylic Material } \\
\text { ABS } \\
\text { ABS-M30i }\end{array}$ \\
\hline Weight & $\begin{array}{l}316 \mathrm{~L}=18.88 \mathrm{~g} \\
\mathrm{RDG} 720=2.37 \mathrm{~g}\end{array}$ & $\begin{array}{l}\text { RDG } 720=6.35 \mathrm{~g} \\
\text { ABS }=5.50 \mathrm{~g} \\
\text { ABS-M30i }=5.35 \mathrm{~g}\end{array}$ \\
\hline
\end{tabular}

implant capsule, Fig. 5.14(left) and the material's interaction with the epoxy and silicone used. This printer will be used to print bio-compatible Titanium in future revisions of the implant capsule to create a stronger lighter weight implant enclosure. A summary of the 3D capsules printed along with the weight of each in Table 5.3 .

Thus, in the following subsection the evaluation results of four different packages for their ability to prevent moisture from penetrating the implant cavity are presented.

\subsubsection{Implant Capsule Water Bath Test}

In order to emulate the effects of a typical implant environment on the prototype, a water bath immersion study is performed. All implants are prepared with the same capsule preparation procedure outlined in Section 5.2.2 and placed into a temperature controlled water bath, Fig. 5.15. Each capsule is opened to determine the effects of water on the implant over short (one week) and long-term (98 days) exposure to water.

\section{Implant Capsule Preparation}

The implant capsules are first cleaned with water to remove any debris that may have formed on the capsules during the $3 \mathrm{D}$ printing and post-processing phases. The $5 \mathrm{~F}$ size catheters are placed inside the catheter opening of the implant and sealed with Loctite 4311 epoxy to secure 


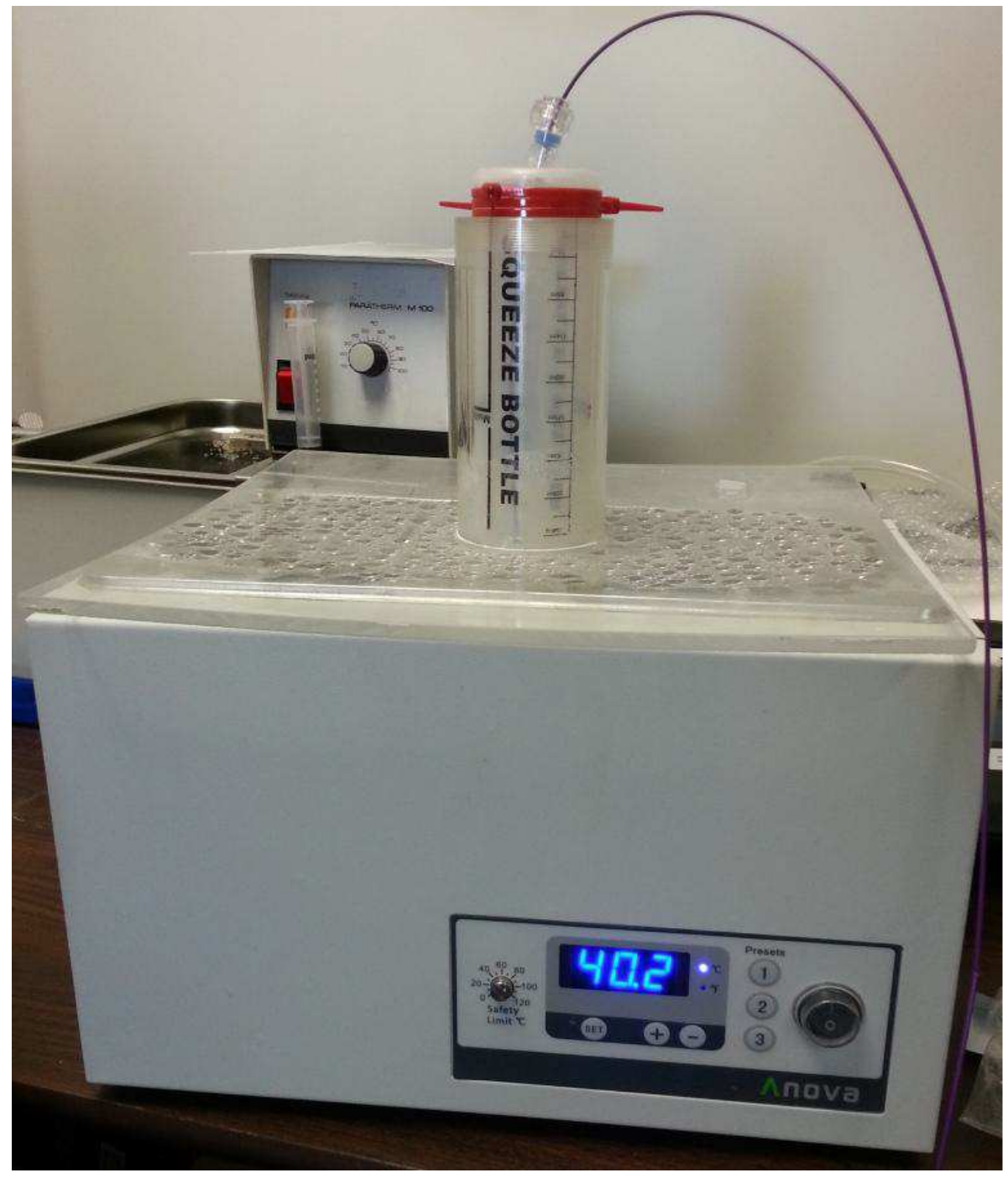

Figure 5.15: Experimental test setup - six liter water bath set to $(40 \pm 0.2)^{\circ} \mathrm{C}$, implants are submersed into water and held there for the duration of the study. 
the catheter inside the implant and to prevent entry to the inner cavity. The process is repeated for the exterior of the catheter entrance. In addition, the catheter tip and end points are sealed to prevent air from traveling into the implant cavity. In this experiment the antenna is not included, the antenna port's main purpose is to provide a viewing port for the included water sensitive paper, thus the antenna access hole is also sealed with Loctite epoxy. Subsequently, the catheter and antenna are UV cured two times for a duration of 30 seconds each.

Chlorophenol detection paper is used to detect if any water enters the internal cavity of the implant capsule. The water detection paper is cut into strips and placed into each capsule half, top and bottom, Fig. 5.16 (a). The two halves of the implant capsule are then sealed with Loctite epoxy and UV cured for 1 minute, rotating the implant throughout to ensure a uniform cure of the capsule. The internal cavity of the implant is isolated from water entry due to the epoxy however, if it or the enclosure itself were to fail, the implant itself is not protected from its environment, thus it must be encapsulated. The implant capsule is dipped into a large volume of Loctite 5091 silicone three times and hung for 10 minutes to remove excess silicone from the capsule. Subsequently, the implant is cured for 2.5 minutes at multiple angles to create a full bio-compatible sealed implant capsule, Fig.5.16(b). A close up of the transparent acrylic material with internal chlorophenol water strips is shown in Fig. 5.17 .

\section{Experimental Setup}

A six liter water bath is set to $40 \pm 0.2^{\circ} \mathrm{C}$, which is typical animal body temperature, Fig. 5.15. Each implant is placed into the water bath and weighted down to prevent it from floating. The Stainless Steel packaged is tied down to prevent the heavier implant from sinking to the bottom of the container. The external view of the water bath setup along with the internal view of the weighted down implants are shown in Fig. 5.15 and 5.18, respectively.

Two studies are completed, a long-term 98 day study and a short-term 1 week test. The long-term study consists of the ABS printed implant only and the remaining three materials are tested in the short term study. The internal Chlorophenol detection paper is monitored through 


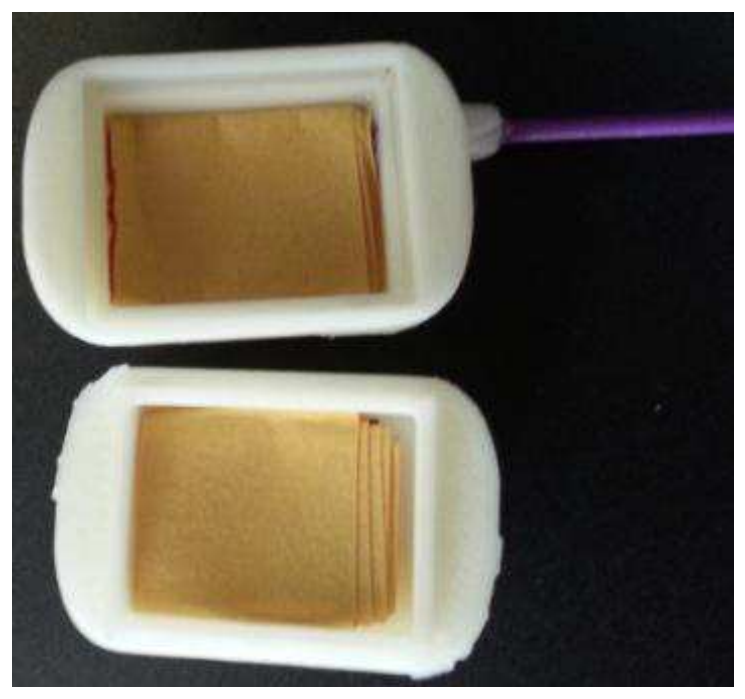

(a)

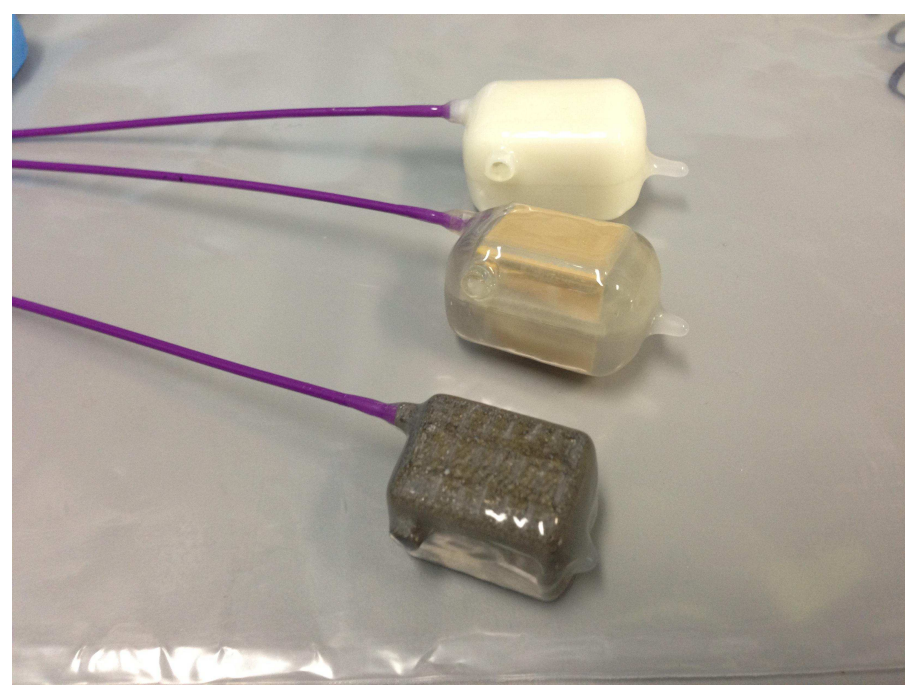

(b)

Figure 5.16: (a) Chlorophenol water test strips placed inside top and bottom cavities of implant capsule. The paper turns purple when in contact with water. (b) Fully sealed implant capsules with Loctite 4311 epoxy and Loctite 5091 silicone.

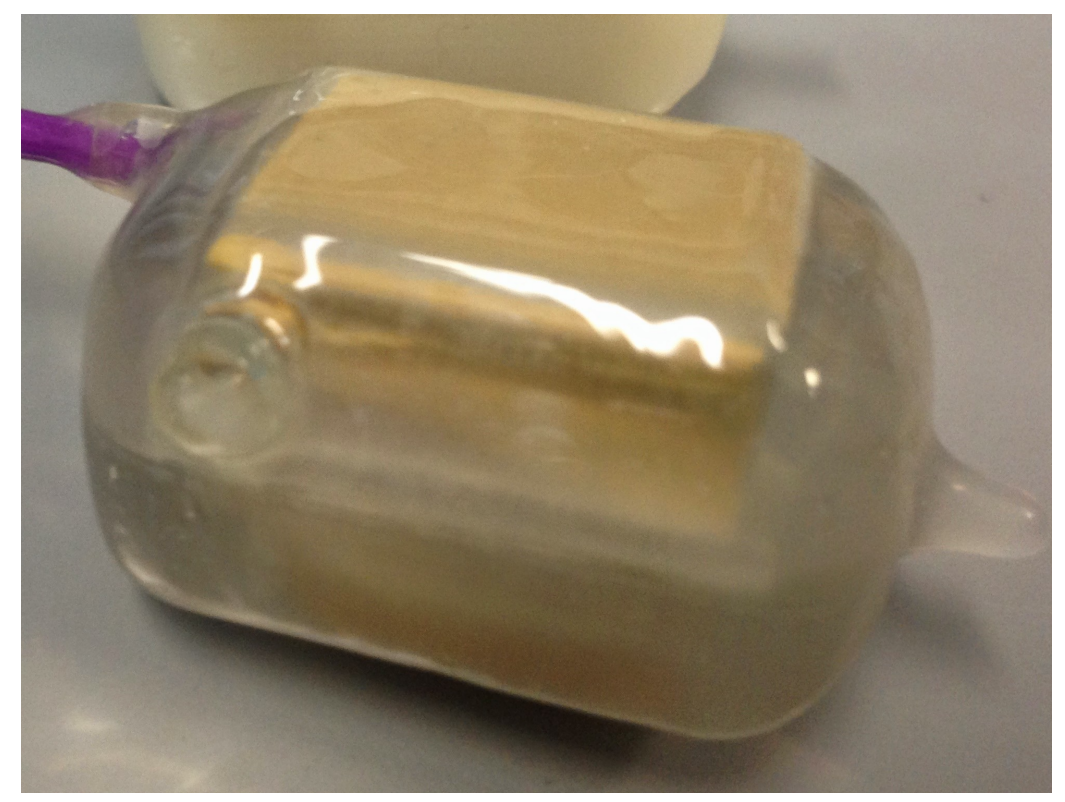

Figure 5.17: A closeup of the thick walled capsule 3D printed in RDG720 transparent acrylic material displaying internal chlorophenol water strips. 


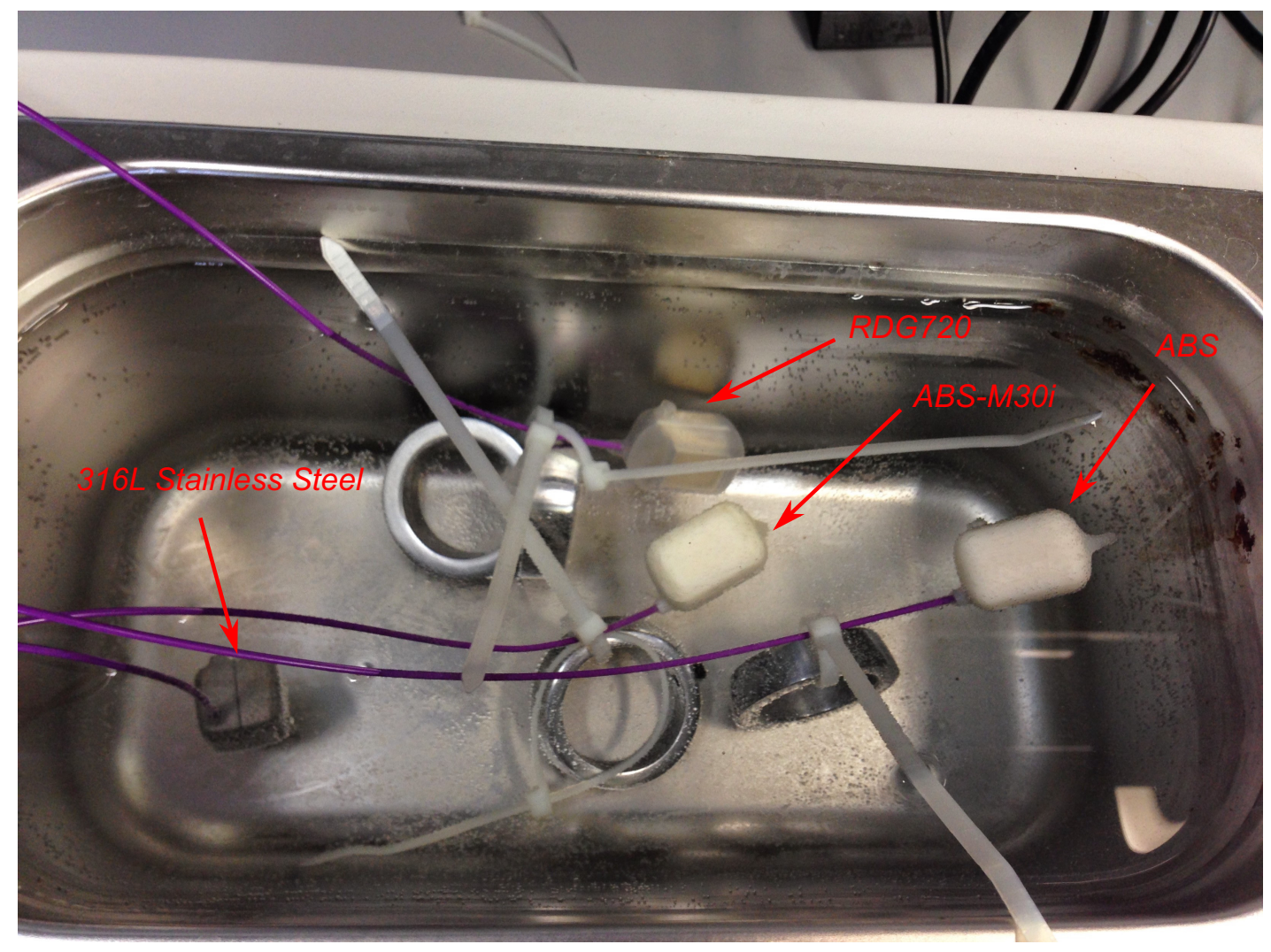

Figure 5.18: Internal view of water bath setup with each implant weighted down to prevent floating. 
the antenna port hole or through the transparent material. The water bath is regularly refilled due to the evaporation of the water.

\section{Water Bath Results}

The detection paper was initially checked daily in the early stages of the the long-term study, however, after 1 week the detection was monitored on a week by week basis. After 98 days, to coincide with the end of the short-term test, the ABS capsule was opened to reveal no noticeable moisture inside the package. Due to the length of the study, the detection paper began to dehydrate, noted by the white locations on the paper, Fig. 5.19 (far right).

Throughout the short-term study, the detection paper was monitored through the antenna port hole four times daily for the one week period. After 7 days the capsules were opened to determine the outcome of the experiment. The RDG720 transparent Acrylic material yielded similar results to the ABS package; no visible moisture was found on the detection paper, Fig. 5.19. The ABS-M30i contained noticeable moisture localized to the region near the antenna port; the remaining Chlorophenol detection paper was unaffected, Fig. 5.19. It is hypothesized that the failure point was due to the curing process of the Loctite 4311 near the location of the antenna port and not due to the design of the capsule or the material selected as the moisture was minor and localized.

The 3D printed stainless steal package yielded much different results in comparison to the plastic capsules. After one day of water immersion, the Stainless Steel began to react on the exterior of the package, Fig. 5.20, although the package is sealed the reaction still occurred. The metal package was placed inside a quarantine cylinder to prevent possible contamination from the other plastic capsules in the water bath for the remainder of the test. The reaction continued on the package outer walls and took approximately 1 day for new reaction locations to form. Inspection of the Chlorophenol detection paper determined that no water moisture transferred through the epoxy barrier into the implant cavity.

All four materials provided sufficient water protection for in-vivo experiments however, 


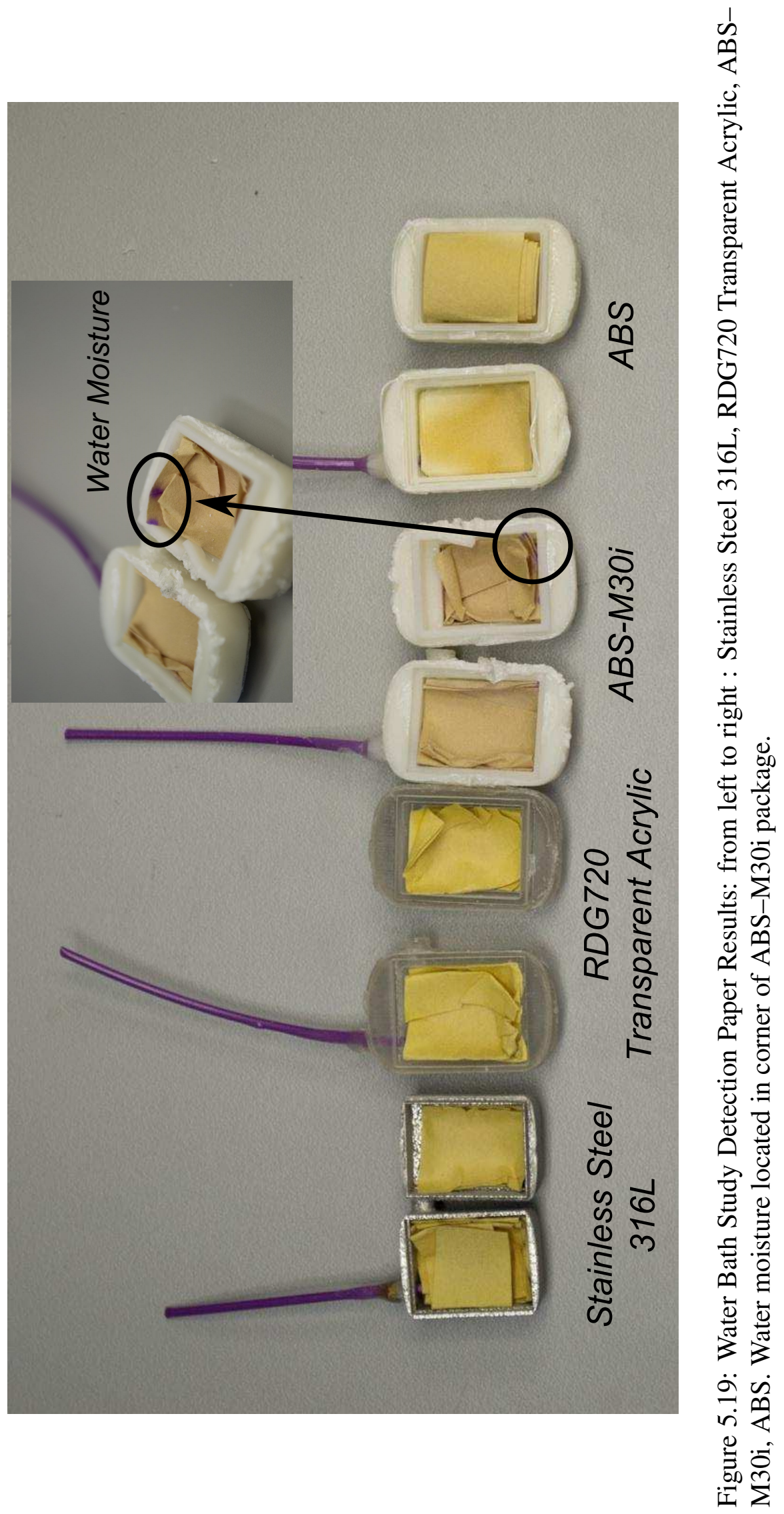




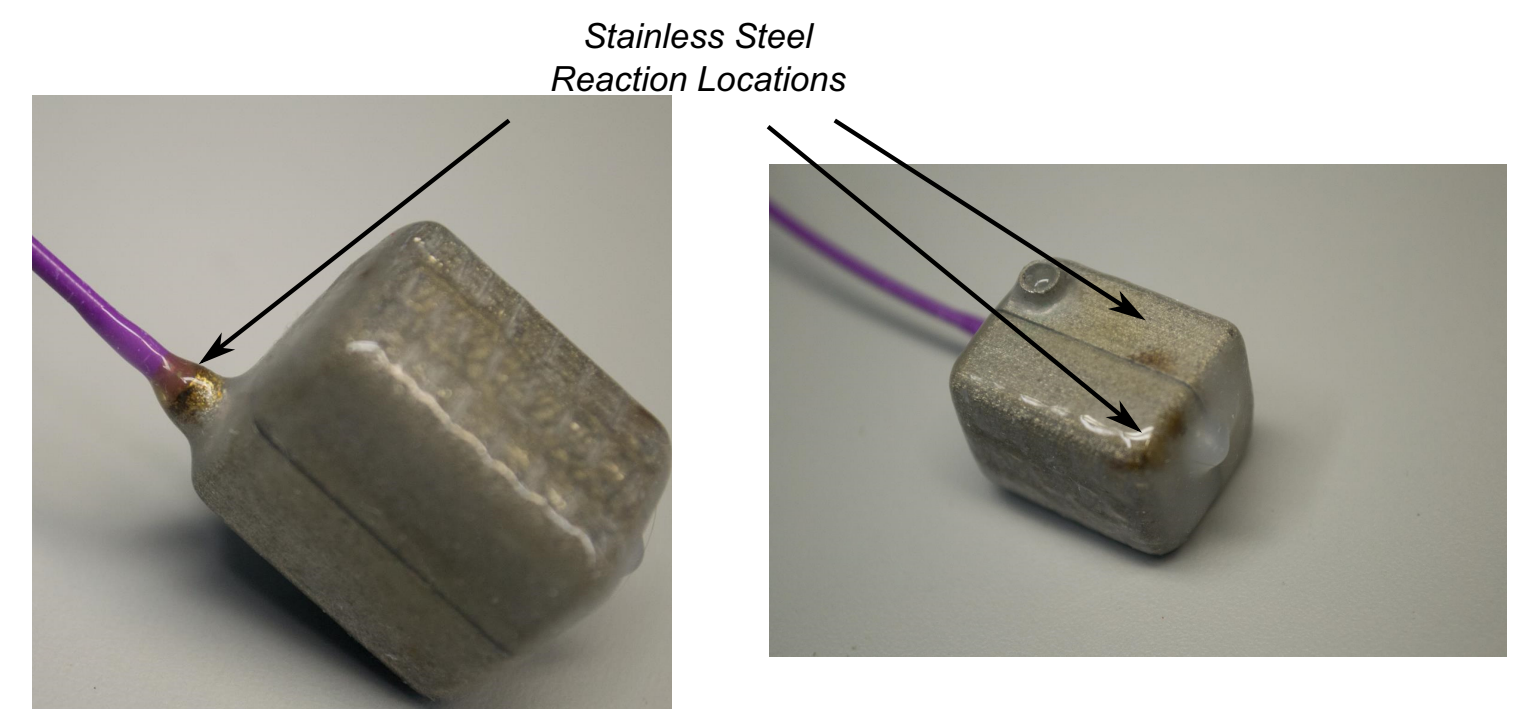

Figure 5.20: Stainless Steel 316L reaction locations at the catheter port hole and along the exterior shell.

additional material interaction studies are required to determine the cause of the reaction on the stainless steel package and the exact failure point of the ABS-M30i package, subject of future work. Furthermore, it can be hypothesized that the reaction most likely occurred due to the stainless steel not being completely polished leaving impurities located on the exterior of the enclosure. It has been shown that $316 \mathrm{~L}$ stainless steel is safe to be used inside the body [265, 266] and thus is mostly an issue of the post-processing of the $316 \mathrm{~L}$ enclosure. Moreover, the availability of a 3D metallic printer enables us to print stainless steel and other metals with high precision. Most importantly, the 3D metallic printer can also print titanium (Ti) and will allow the design of a complete hybrid implantable package with bio-compatible printable metal for a custom antenna solution and 3D printed plastic for the overall implant structure, subject of future work.

\subsubsection{Enclosure for In-Vivo Study}

For the in-vivo experiment, a modified implant package is designed without the antenna port and with suture holes to allow the implant capsule to be secured to the test subject, Fig. 5.21 This enclosure is based on the thick walled version, Fig. 5.14 to ensure the implant is not 


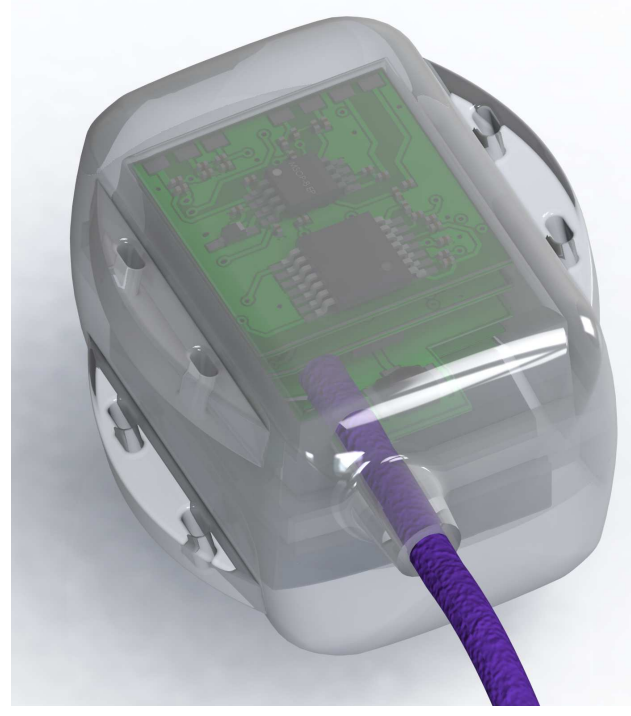

(a)

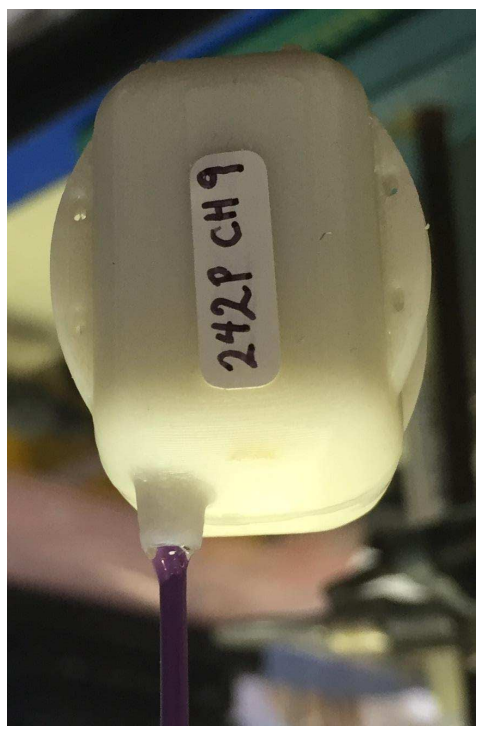

(b)

Figure 5.21: a) 3D rendering of the final package design with internal PCB boards, catheter and suture holes shown; b) 3D printed prototype prior to surgery.

damaged by the weight of the animal while implanted. The antenna port is removed for two reasons. First, it is removed to prevent a possible failure point. As the antenna port must be sealed with epoxy and dipped with silicone, it also must pass the flexible external antenna through. Although, similar to the catheter hole, the antenna is much smaller in diameter and is weaker than the catheter tubing which may lead to not bonding correctly with the epoxy / silicone. Furthermore, the epoxy around the antenna may prevent flexibility on the antenna leading to failure of the antenna or bio material entering the cavity. The second reason for removing the port hole is the external antenna itself. Although the antenna is designed to be flexible, it was observed to bend and in some cases fail when inserted into the phantom tissue. In an in-vivo situation the antenna could bend at the output of the antenna port if forced into tissue and cause significant failure of the implantable system. Therefore, the antenna and antenna port are removed and replaced with an internal PCB antenna, Fig. 4.10(right). This new package is printed in ABS-M30i plastic to ensure full bio-compatibility with the animal subject and is more robust containing less failure points. 


\subsection{PV System Calibration}

This section will discuss the calibration process for both the pressure and volume electronics where each block is calibrated again known loads.

\subsubsection{Pressure Calibration}

The pressure calibration is performed in three main stages. First, the telemetry system and a PC running the ZMDI software are configured. This is followed by the pressure chamber setup and culminating in a fully calibrated MEMS pressure sensor.

\section{Electronic Setup}

The electronic setup consists of: 1) the PV system that requires calibration; 2) the PV system development module and breakout board; 3) ZMDI communication module; and 4) a Windows PC to run the ZMDI software, Fig. 5.22. The PV system with ZMDI ZSC 31015 is connected to the development module via the breakout board as described in Section 4.5

The ZMDI communication module is connected to the development module using four wires: VDD, GND, OWI, and Tr-D2, Fig. 5.23. Power and ground are supplied to the implant via the VDD and GND pins on the communications module and programming the ZDMI IC is achieved with the OWI and Tr-D2 pins. Power to the communications module is supplied through the connected USB cable from the PC and the OWI pin is connected directly to the pressure out pin of on the development module. Recall that the pressure output pin on the ZSC 31015 is also multiplexed with ZMDI's custom one wire interface (OWI), along with additional analog outputs which are used while the implant is in operation. To use the OWI for programming the additional pin, Tr-D2, is required. Tr-D2 is a GPIO pin controlled automatically in the ZMDI software and is connected through a $3.3 \mathrm{k} \Omega$ resistor to the pressure output pin.

When the ZMDI calibration software starts, the user must open the COM port associated with the connected communication module and initialize the hardware. When the user selects 


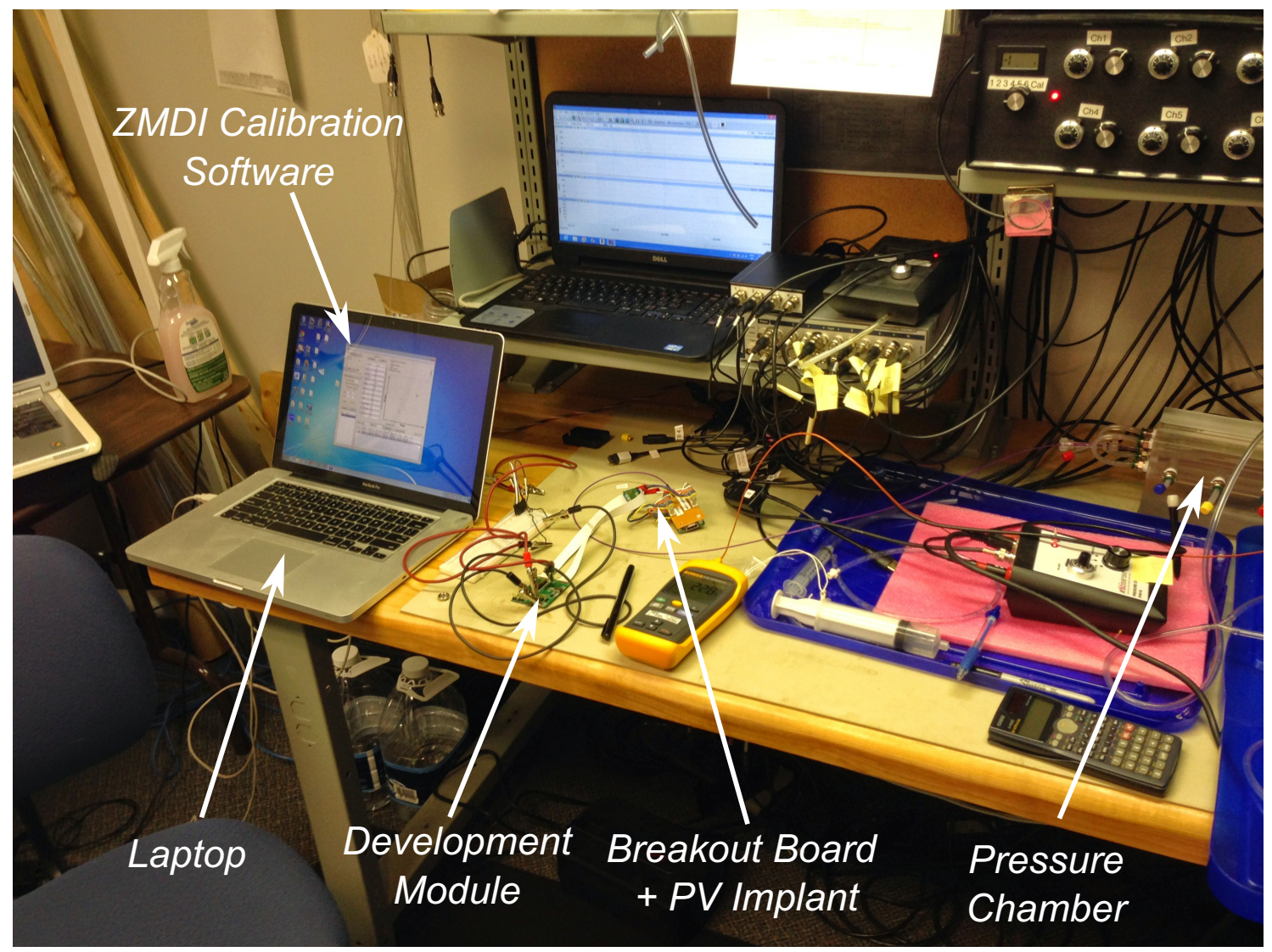

Figure 5.22: Pressure calibration experimental setup. Shown: PV system, Development Module / Breakout board, PC, Software. Not shown: ZMDI Communication Module.

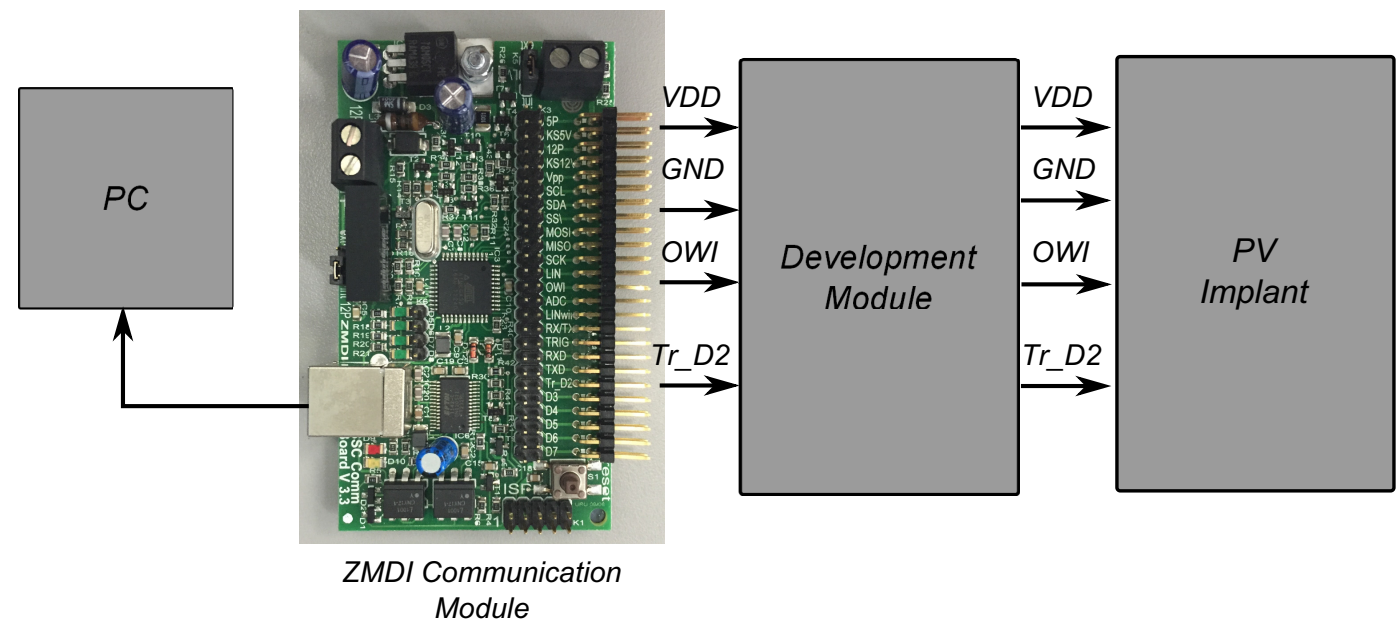

Figure 5.23: ZMDI Communication Module connection to Development module and PV system. 


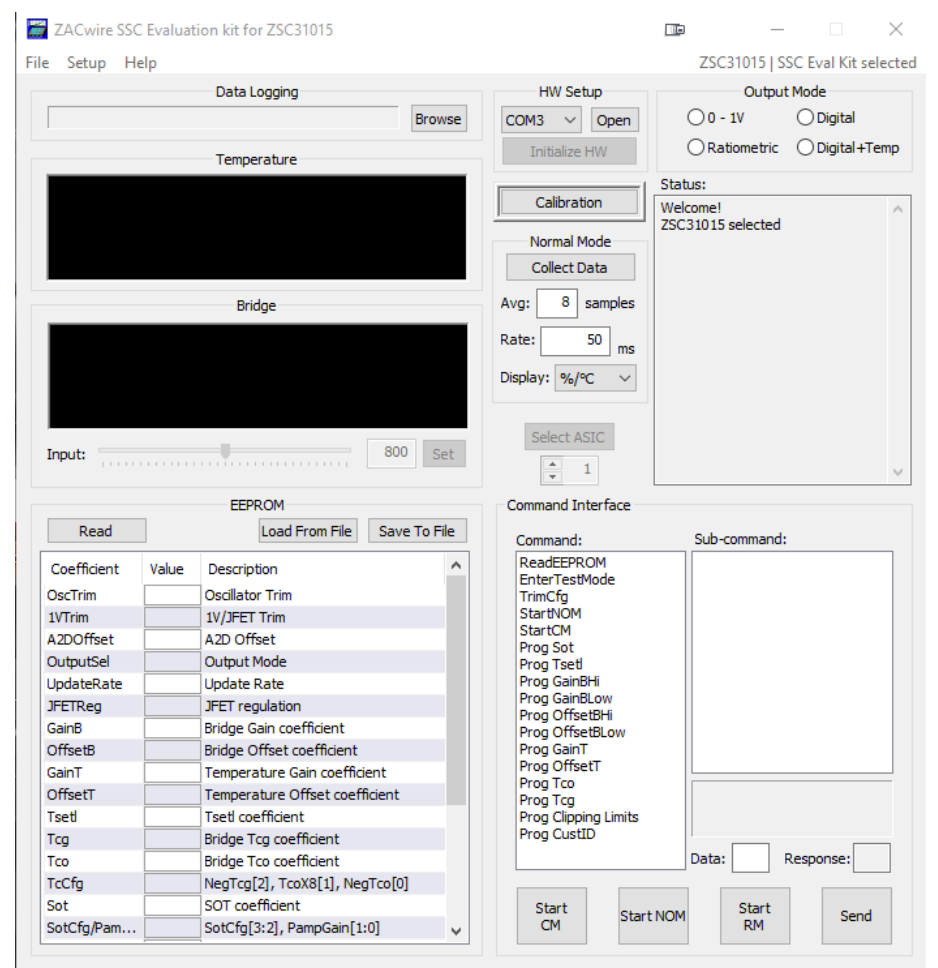

Figure 5.24: ZMDI evaluation software kit software [28]. Copyright @CZMDI.

the initialize hardware button on the ZMDI software, Fig. 5.24 [267], the communication module will pulse the Tr-D2 pin causing the OWI pin to communicate with the ZSC 31015 IC, placing it into read/write mode. At this stage, the user can change the IC's output mode from digital to analog, read the temperature data (if attached) and read the measured voltages. More importantly, the IC can be put into calibration mode, where the specific calibration settings can be changed.

\section{Pressure Calibration Chamber Setup}

The MEMS absolute pressure sensor is calibrated inside a fluid-filled pressure chamber that allows for variations in temperature and pressure, Fig. 5.25. The catheter is inserted into one of the six attached introducers integrated into the chamber. The pressure is controlled via a pneumatic pump and temperature is controlled by cycling water through a separate temperature controlled tank. The pressure is cycled at a very low frequency sub- $\mathrm{Hz}$ for calibration. This is to ensure there is ample time to make measurements in the ZMDI software when changing 


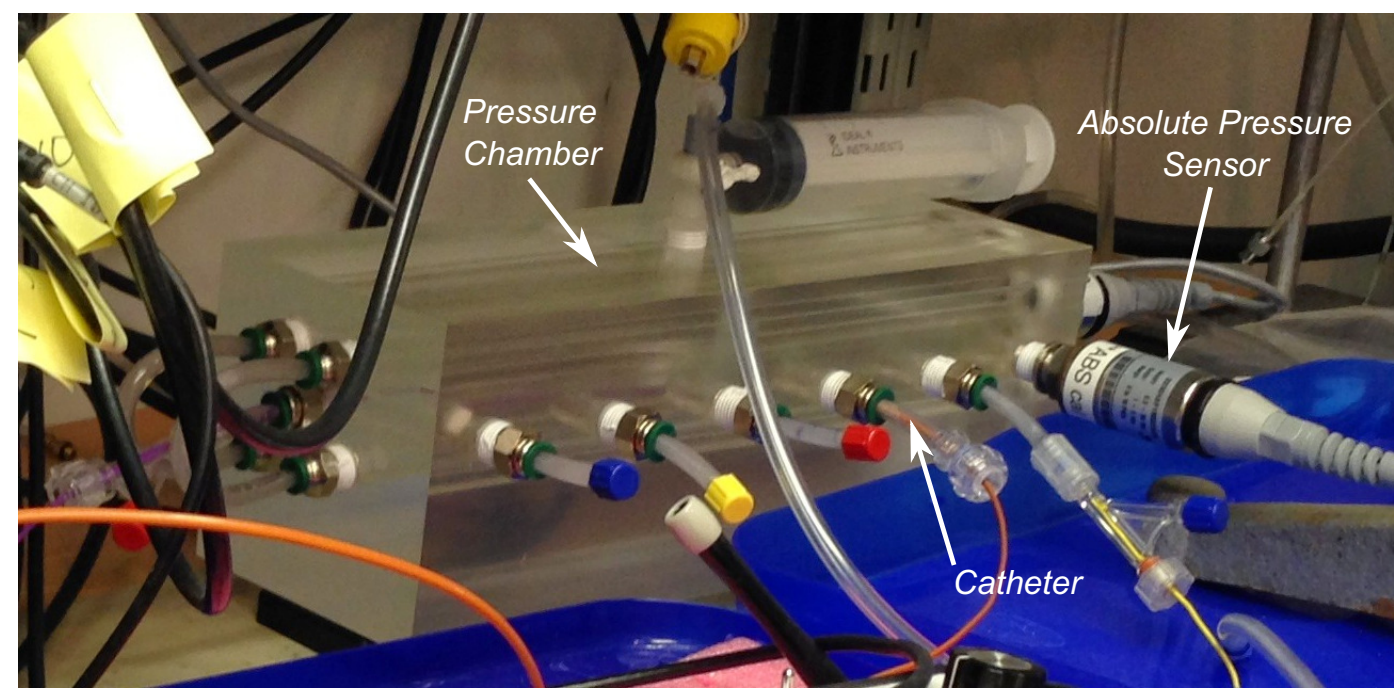

Figure 5.25: Pressure chamber used in pressure calibration.

pressures within the chamber. Temperature change from maximum to minimum typically takes about an hour to cycle through due to the limits of the hardware used. An additional calibrated temperature and pressure sensors are inserted into the chamber to provide an absolute pressure and temperature reference for the calibration process. These sensor are measured on a separate PC running LabScribe [268] measurement software. This setup is used for all pressure calibrations.

\section{Pressure Calibration Process}

Four point calibration is used to calibrate for the variation of the wheatstone bridge offset over temperature and variation of the bridge gain over temperature thereby providing $1^{s t}$ order temperature compensation. All calibration data is stored within the ZMDI ZSC 3105 IC at two temperatures, $22.8^{\circ} \mathrm{C}$ and $43.2^{\circ} \mathrm{C}$ representing a wide range of temperature operation. However, in practice inside the animal, the sensor should see little variation due to the stability of body temperature of $39^{\circ} \mathrm{C}$. The sensor is calibrated over a $450 \mathrm{mmHg}$ pressure range, from $-135 \mathrm{mmHg}$ to $315 \mathrm{mmHg}$. This range allows the pressure sensor to work within the expected physiological range of the swine left ventricle with headroom for atmospheric pressure changes. 
The calibrated parameters are stored on the internal EEPROM located on the ZSC 31015 and are recalled upon startup. The pressure circuit is set to span the complete voltage range of $0 \mathrm{~V}-3.6 \mathrm{~V}$, thus $8 \mathrm{mV}$ per $\mathrm{mmHg}$ with a $-135 \mathrm{mmHg}$ pressure offset, (5.2).

$$
\text { Pressure }=\frac{\text { Voltage Output }}{8 \mathrm{mV} / \mathrm{mmHg}}-135 \mathrm{mmHg}
$$

\subsubsection{Volume Calibration}

The PV interface produces real and imaginary or in-phase and quadrature (I and Q) voltages that are functions of the measured load, in this case the left ventricle blood impedance/conductance. The PV interface is calibrated in two steps: a) creation of a calibration matrix to generate calibration coefficients and b) generation of system phase shift and voltage magnitude calibration curves.

\section{Volume Calibration Setup}

The volume component of the PV sensor interface is calibrated against known RC loads instead of the actual PV catheter. Since the current implementation of the PV interface module is best suited for medium size animal hearts with typical volumes ranging from approximately $35 \mathrm{ml}$ to $85 \mathrm{ml}$, whose corresponding conductance values range between $10 \mathrm{mS}-30 \mathrm{mS}$, resistive loads in the $\mathrm{R}_{L}=30 \Omega-100 \Omega$ range are used. In addition, a variable capacitor value between 0 $\mathrm{nF}-30 \mathrm{nF}$ is used to represent the phase shift of the myocardium. Each known RC "standard" is then connected between electrodes $\mathrm{b}$ and $\mathrm{c}$ of the catheter, Fig. 4.12 of the catheter to emulate the ideal admittance value of the heart. This setup is used throughout the volume calibration process.

\section{Calibration Matrix}

A calibration matrix or transformation matrix is created to transform the measured real and imaginary voltages to the theoretical voltages at the sensor input, 5.3 where $I_{\text {Theo }}, Q_{\text {Theo }}$ rep- 
resent the theoretical voltages at the sensor input, (a) through (d) represent the calibration coefficients and $I_{\text {Meas }}, Q_{\text {Meas }}$ represent the measured voltages of the PV interface output. The theoretical voltages at the sensor input are derived from the excitation current $\mathrm{I}_{\text {Drive }}$, excitation frequency in radians $\omega$ and the known $\mathrm{R}$ and $\mathrm{C}$ load, (5.4), (5.5).

$$
\begin{gathered}
\left(\begin{array}{l}
\mathrm{I}_{\text {Theo }} \\
\mathrm{Q}_{\text {Theo }}
\end{array}\right)=\left(\begin{array}{ll}
\mathrm{a} & \mathrm{b} \\
\mathrm{c} & \mathrm{d}
\end{array}\right)\left(\begin{array}{l}
\mathrm{I}_{\text {Meas }} \\
\mathrm{Q}_{\text {Meas }}
\end{array}\right) \\
I_{\text {Theo }}=\frac{R \cdot I_{\text {Drive }}}{C^{2} \cdot R^{2} \cdot \omega^{2}+1} \\
Q_{\text {Theo }}=-\frac{C \cdot R^{2} \cdot \omega \cdot I_{\text {Drive }}}{C^{2} \cdot R^{2} \cdot \omega^{2}+1}
\end{gathered}
$$

Thus, $I_{\text {Theo }}$ and $Q_{\text {Theo }}$ are both functions of $I_{\text {Meas }}$ and $Q_{\text {Meas }}$. Two separate measurements taken from the PV interface for two different load values will generate (5.6) from the calibration matrix.

$$
\begin{array}{r}
I_{1}=a \cdot I_{\text {Meas } 1}+b \cdot Q_{\text {Meas } 1} \\
Q_{1}=c \cdot I_{\text {Meas } 1}+d \cdot Q_{\text {Meas } 1} \\
I_{2}=a \cdot I_{\text {Meas } 2}+b \cdot Q_{\text {Meas2 }} \\
Q_{2}=c \cdot I_{\text {Meas } 2}+d \cdot Q_{\text {Meas2 }}
\end{array}
$$

$I_{1}, I_{2}, Q_{1}, Q_{2}$ are the theoretical real and imaginary voltages at the sensor interface found by (5.4) and (5.5); $I_{\text {Meas1 } 1}, I_{\text {Meas2 } 2}, Q_{\text {Meas1 }}, Q_{\text {Meas2 }}$ are the measured voltage outputs at the known loads from the PV interface module thus calibration coefficients a, b, c, d are calculated from (5.7) - 5.10).

The calibration coefficients used in the in-vivo experiment are found in Table 5.4 and 5.5 along with the RC loads, excitation current and frequency used to generate the coefficients. 


$$
\begin{aligned}
& a=\frac{I_{\text {Drive }} \cdot\left(Q_{\text {Meas2 }} \cdot C_{2}^{2} \cdot R_{1} \cdot R_{2}^{2} \cdot \omega^{2}-\cdot Q_{\text {Meas } 1} \cdot C_{1}^{2} \cdot R_{1}^{2} \cdot R_{2} \cdot \omega^{2}+Q_{\text {Meas } 2} \cdot R_{1}-Q_{\text {Meas } 1} \cdot R_{2}\right)}{\left(I_{\text {Meas } 1} \cdot Q_{\text {Meas } 2}-I_{\text {Meas2 }} \cdot Q_{\text {Meas1 }}\right) \cdot\left(C_{1}{ }^{2} \cdot R_{1}{ }^{2} \cdot \omega^{2}+1\right) \cdot\left(C_{2}^{2} \cdot R_{2}^{2} \cdot \omega^{2}+1\right)} \\
& b=-\frac{I_{\text {Drive }} \cdot\left(I_{\text {Meas } 2} \cdot C_{2}^{2} \cdot R_{1} \cdot R_{2}^{2} \cdot \omega^{2}-\cdot I_{\text {Meas } 1} \cdot C_{1}^{2} \cdot R_{1}^{2} \cdot R_{2} \cdot \omega^{2}+I_{\text {Meas } 2} \cdot R_{1}-I_{\text {Meas } 1} \cdot R_{2}\right)}{\left(I_{\text {Meas } 1} \cdot Q_{\text {Meas } 2}-I_{\text {Meas } 2} \cdot Q_{\text {Meas } 1}\right) \cdot\left(C_{1}^{2} \cdot R_{1}^{2} \cdot \omega^{2}+1\right) \cdot\left(C_{2}^{2} \cdot R_{2}^{2} \cdot \omega^{2}+1\right)} \\
& c=-\frac{I_{\text {Drive }} \cdot \omega \cdot\left(Q_{\text {Meas } 2} \cdot C_{1} \cdot C_{2}{ }^{2} \cdot R_{1}{ }^{2} \cdot R_{2}{ }^{2} \cdot \omega^{2}-\cdot Q_{\text {Meas } 1} \cdot C_{1}{ }^{2} \cdot C_{2} \cdot R_{1}{ }^{2} \cdot R_{2}{ }^{2} \cdot \omega^{2}+Q_{\text {Meas2 }} \cdot C_{1} \cdot R_{1}{ }^{2}-Q_{\text {Meas } 1} \cdot C_{2} \cdot R_{2}\right)^{2}}{\left(I_{\text {Meas } 1} \cdot Q_{\text {Meas2 }}-I_{\text {Meas2 } 2} \cdot Q_{\text {Meas } 1}\right) \cdot\left(C_{1}{ }^{2} \cdot R_{1}{ }^{2} \cdot \omega^{2}+1\right) \cdot\left(C_{2}{ }^{2} \cdot R_{2}{ }^{2} \cdot \omega^{2}+1\right)} \\
& d=\frac{I_{\text {Drive }} \cdot \omega \cdot\left(I_{\text {Meas } 2} \cdot C_{1} \cdot C_{2}{ }^{2} \cdot R_{1}{ }^{2} \cdot R_{2}{ }^{2} \cdot \omega^{2}-\cdot I_{\text {Meas } 1} \cdot C_{1}{ }^{2} \cdot C_{2} \cdot R_{1}{ }^{2} \cdot R_{2}{ }^{2} \cdot \omega^{2}+I_{\text {Meas } 2} \cdot C_{1} \cdot R_{1}{ }^{2}-I_{\text {Meas } 1} \cdot C_{2} \cdot R_{2}\right)^{2}}{\left(I_{\text {Meas } 1} \cdot Q_{\text {Meas } 2}-I_{\text {Meas } 2} \cdot Q_{\text {Meas } 1}\right) \cdot\left(C_{1}{ }^{2} \cdot R_{1}{ }^{2} \cdot \omega^{2}+1\right) \cdot\left(C_{2}{ }^{2} \cdot R_{2}{ }^{2} \cdot \omega^{2}+1\right)}
\end{aligned}
$$

Table 5.4: In-Vivo Calibration Coefficients for PV Volume Interface.

\begin{tabular}{l|l|l|l}
\hline $\mathrm{a}$ & $\mathrm{b}$ & $\mathrm{c}$ & $\mathrm{d}$ \\
\hline $1.07 \mathrm{E}-3$ & $1.09 \mathrm{E}-3$ & $-1.16 \mathrm{E}-3$ & $6.21 \mathrm{E}-05$ \\
\hline
\end{tabular}

Table 5.5: In-Vivo Calibration Data for PV Volume Interface.

\begin{tabular}{l|l|l|l|l|l}
\hline$R_{1}[\Omega]$ & $R_{2}[\Omega]$ & $C_{1}[\mathrm{nF}]$ & $C_{2}[\mathrm{nF}]$ & $I_{\text {Drive }}[\mu \mathrm{A}]$ & Frequency $[\mathrm{kHz}]$ \\
\hline 33 & 100 & 0 & 30 & 46.5 & 20 \\
\hline
\end{tabular}

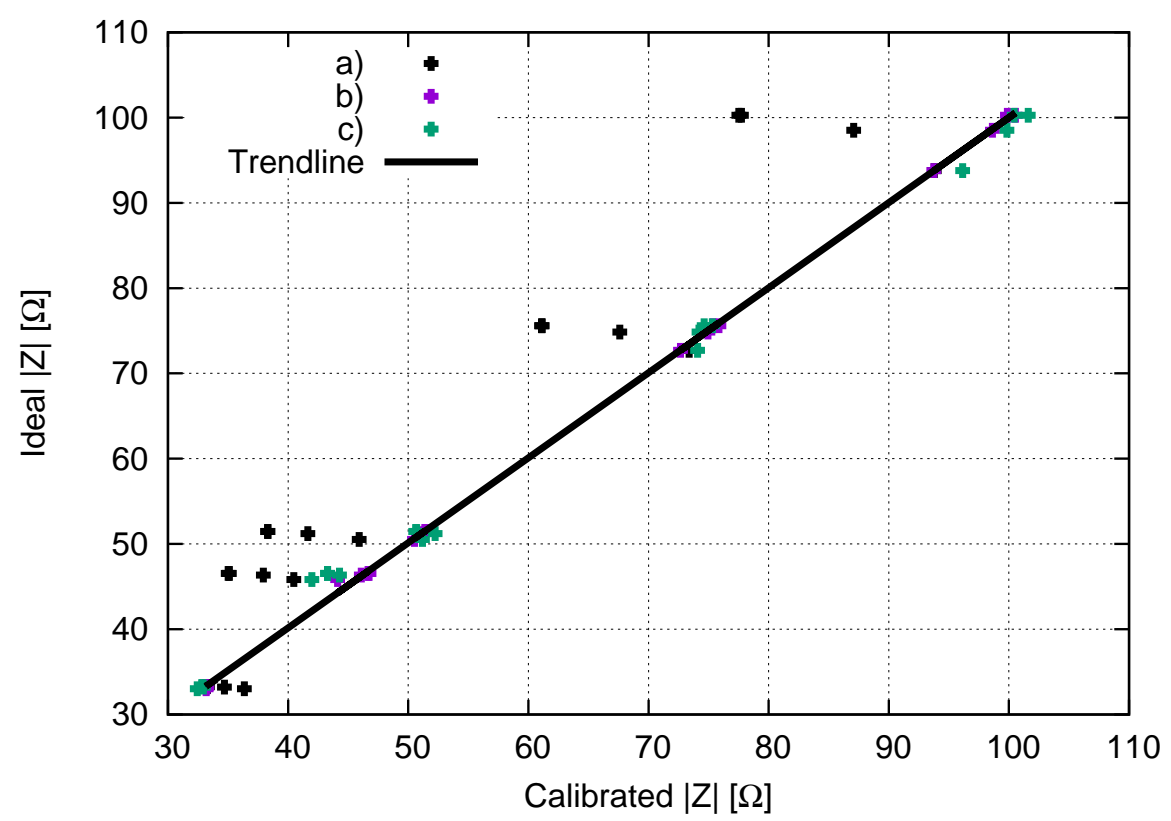

Figure 5.26: Ideal $|Z|$ vs a) $|Z|_{\text {Calc }}$; b) $|Z|_{\text {Cal }}$ Method 1; c) $|Z|_{\text {Cal }}$ Method 2. The trend line is also shown. 


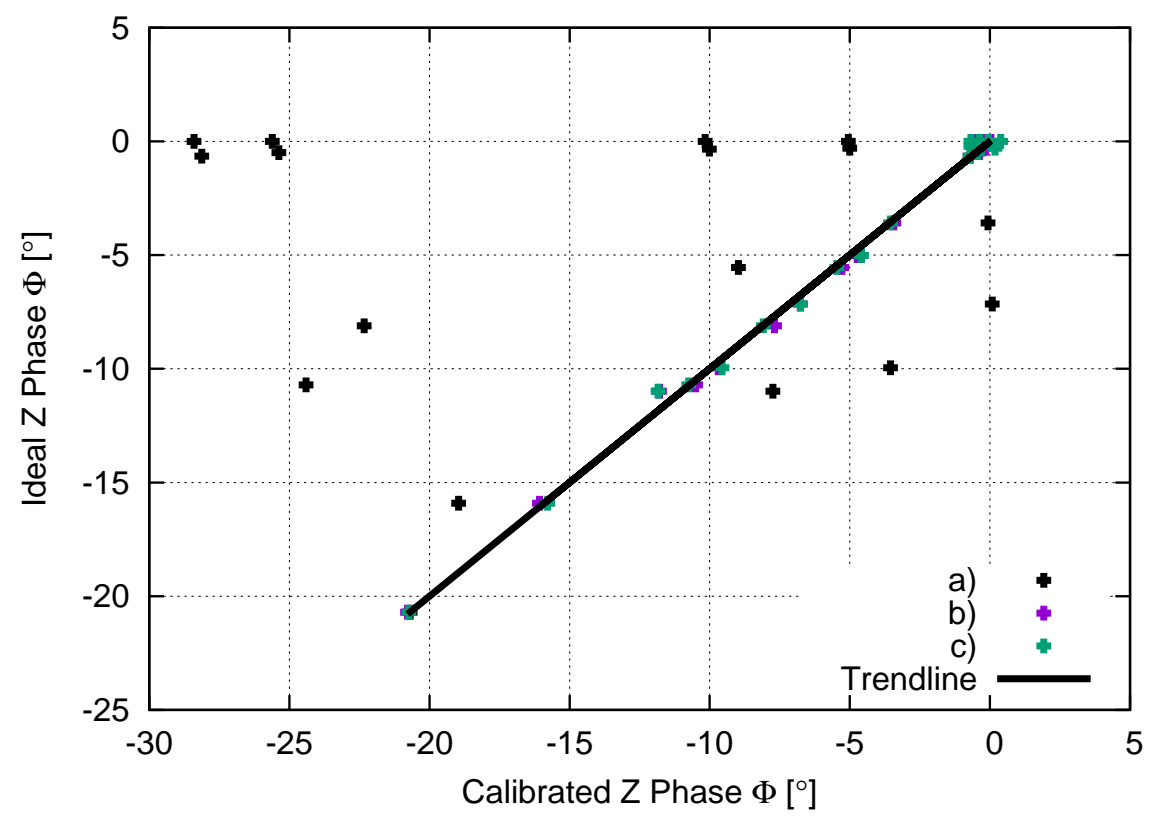

Figure 5.27: Ideal $\phi_{Z}$ vs a) $\phi_{\text {Calc }}$; b) $\phi_{\mathrm{ZCal}}$ Method 1 ; c) $\phi_{\mathrm{ZCal}}$ Method 2. The trend line is also shown.

\section{Calibration Curves}

Although, the calibration matrix transforms the measured output voltages to calculated values at the sensor input, the matrix is truly only accurate at the two calibration points, $|Z|=33 \Omega$ and $|Z|=93.79 \Omega$, due to the inherent phase shift of the system, $\phi_{\text {System }}$, Fig 5.26a and Fig 5.27a, where $|Z|_{\text {Calc }}$ and $\phi_{\text {Calc }}$ are the calculated magnitude and phase of the impedance calculated from the calculated output voltages from the calibration matrix with no additional calibration across the calibration range. The system phase shift is measured by 5.11

$$
\phi_{\text {System }}=\phi_{\text {Ideal }}-\phi_{\text {Calc }}
$$

where $\phi_{\text {Ideal }}$ and $\phi_{\text {Calc }}$ are the ideal and calculated phase of the voltage signal at the sensor for each load point. The system phase shift, $\phi_{\text {System }}$, is not constant throughout the calibration range, i.e, different loads will have different system phase shifts. More precisely, $\phi_{\text {System }}$ is a function of the load itself, Fig. 5.28 and must be calibrated out to ensure accurate impedance measurements. 


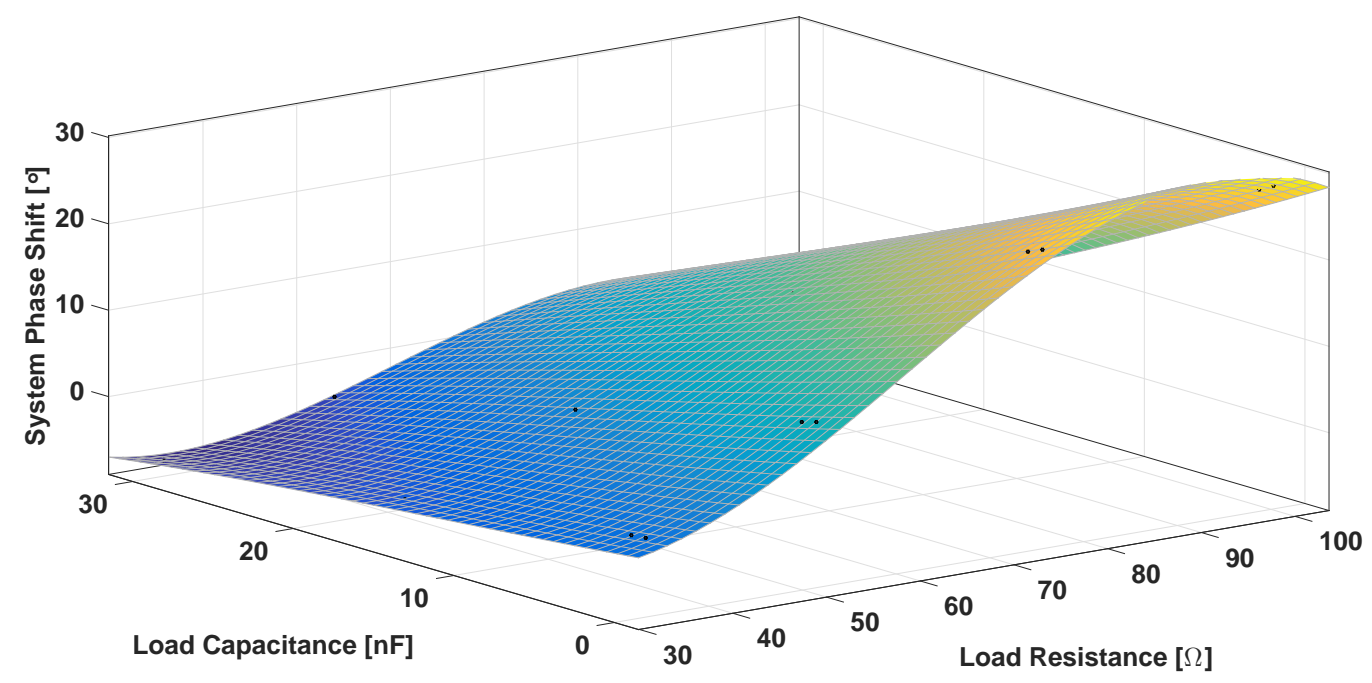

Figure 5.28: Ideal Load Resistance vs Ideal Load Capacitance vs System Phase Shift $\phi_{\text {System }}$.

However, as the impedance is the intended signal of measurement, the exact relationship to phase shift cannot be calculated directly with just the voltage values calculated at the sensor input. Instead, solved $R_{\text {Calc }}$ and $C_{\text {Calc }}$ values from (5.12) and (5.13), where $I_{\text {Calc }}, Q_{\text {Calc }}$ are the calculated voltages of the sensor input after matrix transformation, are used to calculate the the system phase shift. Similar to the ideal case, the relationship between $R_{\text {Calc }}, C_{\text {Calc }}$, and $\phi_{\text {System }}$ is found such that the system phase shift at any load point can be determined, Fig. 5.29.

$$
\begin{gathered}
a \cdot I_{\text {Meas }}+b \cdot Q_{\text {Meas }}=I_{\mathrm{Calc}}=\frac{R_{\mathrm{Calc}} \cdot I_{\text {Drive }}}{C_{\mathrm{Calc}}{ }^{2} \cdot R_{\mathrm{Calc}}{ }^{2} \cdot \omega^{2}+1} \\
c \cdot I_{\text {Meas }}+d \cdot Q_{\text {Meas }}=Q_{\mathrm{Calc}}=-\frac{C_{\mathrm{Calc}} \cdot R_{\mathrm{Calc}}{ }^{2} \cdot \omega \cdot I_{\text {Drive }}}{C_{\mathrm{Calc}}{ }^{2} \cdot R_{\mathrm{Calc}}^{2} \cdot \omega^{2}+1}
\end{gathered}
$$

A $3^{r d}$ order xy polynomial fit 5.15 is used to approximate the surface where $R_{\text {Curve }}$ and $C_{\text {Curve }}$ are normalized by the mean and standard deviation of their respected variables $R_{\text {Calc }}$,

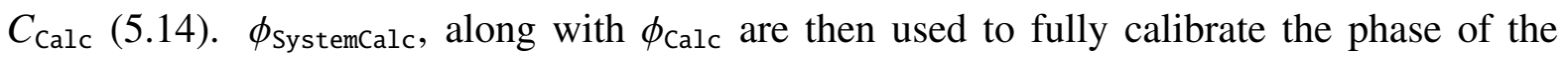
measured signal, $\phi_{\text {Cal }}$ 5.16. 


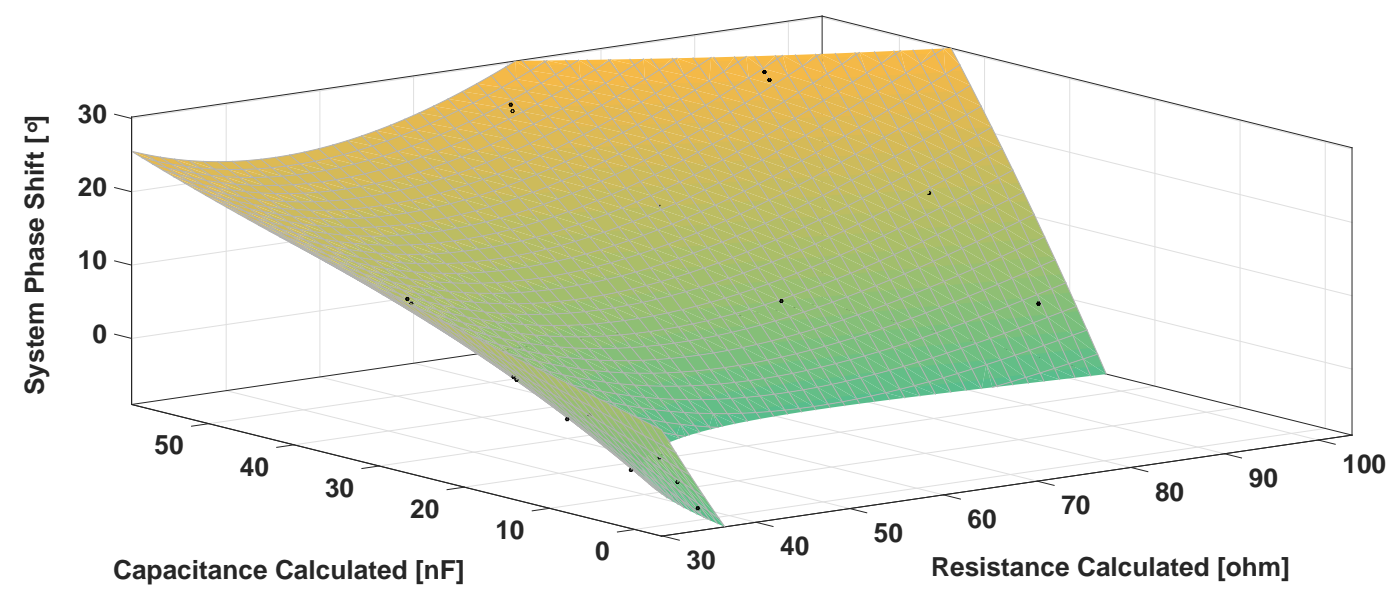

Figure 5.29: Calculated Resistance $\mathrm{R}_{\text {Calc }}$ vs Calculated Capacitance $\mathrm{C}_{\text {Calc }}$ vs System Phase Shift $\phi_{\text {System }}$.

$$
\begin{gathered}
R_{\text {Curve }}=\frac{R_{\text {Calc }}-54.49}{25.99} \\
C_{\text {Curve }}=\frac{C_{\text {Calc }}-32.05 e^{-8}}{14.78 e^{-8}} \\
\phi_{\text {Systemcalc }}=-4.862+\left(-4.95 * R_{\text {Curve }}+\left(21.19 * C_{\text {Curve }}\right)+\left(7.515 * R_{\text {Curve }}{ }^{2}\right)+\left(12.58 * R_{\text {Curve }} * C_{\text {Curve }}\right)\right. \\
+\left(-1.687 * C_{\text {Curve }^{2}}\right)+\left(-1.386 * R_{\text {Curve }}{ }^{3}\right)+\left(-0.1469 * R_{\text {Curve }}{ }^{2} * C_{\text {Curve }}\right) \\
+\left(-1.692 * R_{\text {Curve }} * C_{\text {Curve }}{ }^{2}\right)+\left(0.1044 * C_{\text {Curve }}{ }^{3}\right)
\end{gathered}
$$$$
\phi_{\mathrm{VCal}}=\phi_{\text {Calc }}+\phi_{\text {SystemCalc }}
$$

The voltage magnitude is also calibrated with a similar process to the phase. The calculated voltage magnitude at the sensor is subtracted from the ideal voltage magnitude to obtain the magnitude difference at each calibration point (5.17).

$$
|V|_{\text {Difference }}=|V|_{\text {Ideal }}-|V|_{\text {Calc }}
$$

As with the phase shift, the magnitude difference is used along with $R_{\text {Calc }}$ and $C_{\text {Calc }}$ to 


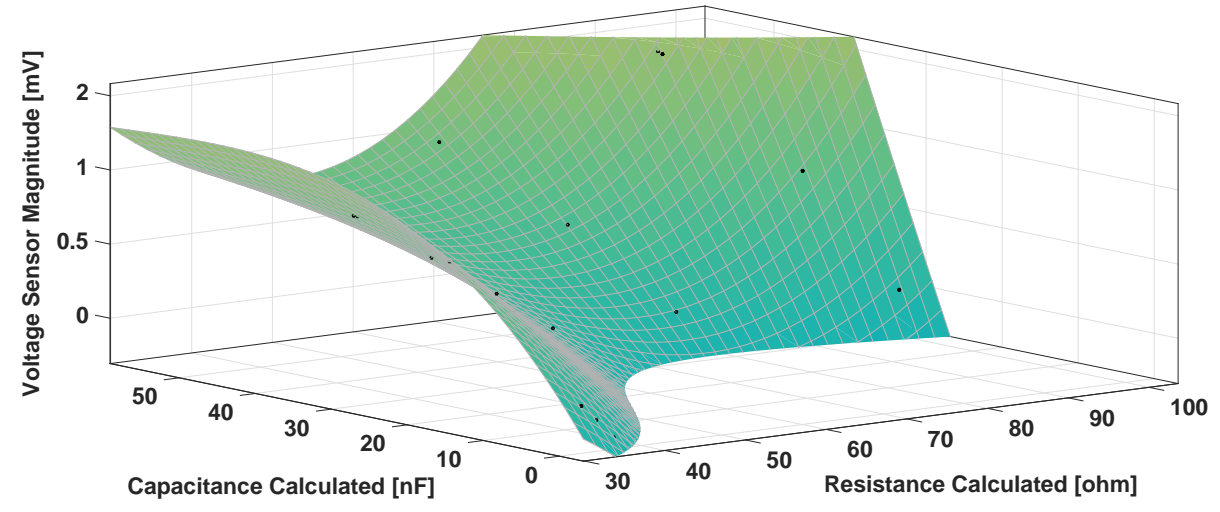

Figure 5.30: Calculated Resistance $\mathrm{R}_{\text {Calc }}$ vs Calculated Capacitance $\mathrm{C}_{\text {Calc }}$ vs $|V|_{\text {Difference }}$.

create a surface fit, Fig. 5.30 A $3^{\text {rd }}$ order xy polynomial fit 5.18 used to approximate the surface, $R_{\text {Curve }}$ and $C_{\text {Curve }}$ are calculated in (5.14)

$$
\begin{aligned}
|V|_{\text {DifferenceCalc }} & =-124.5 E^{-6}+\left(-751.9 E^{-6} * R_{\text {Curve }}\right)+\left(656.8 E^{-6} * C_{\text {Curve }}\right)+\left(411.4 E^{-6} * R_{\text {Curve }}{ }^{2}\right) \\
& +\left(725.6 E^{-6} * R_{\text {Curve }} * C_{\text {Curve }}\right)+\left(-8.016 E^{-5} * R_{\text {Curve }} * C_{\text {Curve }}{ }^{2}\right)+\left(101.2 E^{-6} * R_{\text {Curve }}{ }^{3}\right) \\
& +\left(317.4 E^{-6} * R_{\text {Curve }}{ }^{2} * C_{\text {Curve }}\right)+\left(9.603 E^{-5} * R_{\text {Curve }} * C_{\text {Curve }}{ }^{2}\right)+\left(4.296 E^{-5} * C_{\text {Curve }^{3}}\right)
\end{aligned}
$$

For each calibration point the calculated $|V|_{\text {Differencecalc }}$ is added to $|V|_{\text {Calc }}$ to obtain the calibrated $|V|_{\text {cal }}$ at the sensor input (5.19).

$$
|V|_{\text {Cal }}=|V|_{\text {DifferenceCalc }}+|V|_{\text {Calc }}
$$

Thus, the calibrated impedance is calculated with $|V|_{\mathrm{Cal}}, \phi_{\mathrm{vCal}}$ and $I_{\text {Drive. }}$ The average phase error across the calibration range is $0.2^{\circ}$ with a strong linear correlation with a $\mathrm{R}^{2}$ value of 0.9983 compared to the ideal phase, $\phi_{\text {Ideal }}$, expected, Fig. 5.27p. The average impedance magnitude error across the calibration is $0.211 \Omega$ with a $\mathrm{R}^{2}$ value of 0.9998 compared to the ideal impedance magnitude, $|V|_{\text {Ideal }}$, expected, Fig. 5.26 $\mathrm{p}$.

Although, the above calibration method is very accurate in obtaining calibrated impedance 


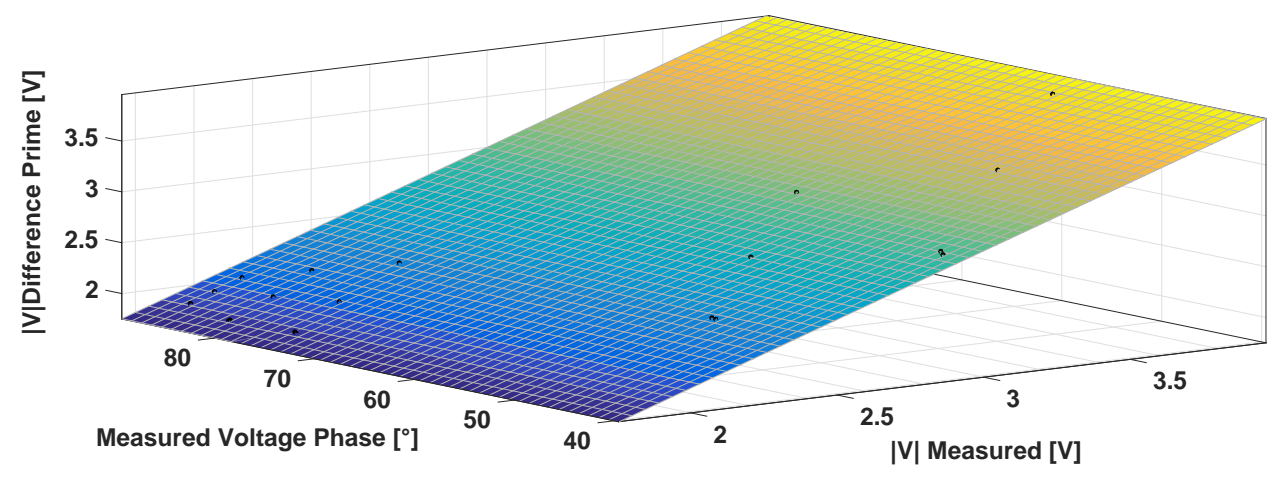

Figure 5.31: Measured Voltage Magnitude $|V|_{\text {Measured }}$ vs Measured Voltage Phase $\phi_{\text {Measured }}$ vs $|V|_{\text {Difference' }}$.

values, the process to solve $R_{\text {Calc }}$ and $C_{\text {Calc }}$ is computationally intensive. A secondary calibration method that does not involve $R_{\mathrm{Calc}}$ and $C_{\mathrm{Calc}}$ is developed. This method involves mapping $|V|_{\text {Measured }}$ and $\phi_{\text {Measured }}$ (5.20) t5.21 to $|V|_{\text {Difference' }}$ and $\phi_{\text {System' }}$, respectively. Recall, $|V|_{\text {Measured }}$ and $\phi_{\text {Measured }}$ are the direct outputs of the PV system.

$$
\begin{aligned}
|V|_{\text {Measured }} & =\sqrt{I_{\text {Meas }}^{2}+Q_{\text {Meas }}^{2}} \\
\phi_{\text {Measured }} & =\tan ^{-1} \frac{Q_{\text {Meas }}^{2}}{I_{\text {Meas }}^{2}}
\end{aligned}
$$

Note that $|V|_{\text {Difference' }}$ and $\phi_{\text {System }}$ are different calculations (5.22) (5.23) in this calibration method compared to the previous method due to the values they are calibrated to, hence, the prime notation.

$$
\begin{gathered}
|V|_{\text {Difference }}=|V|_{\text {Measured }}-|V|_{\text {Ideal }} \\
\phi_{\text {System }^{\prime}}=\phi_{\text {Measured }}-\phi_{\text {Ideal }}
\end{gathered}
$$

Similar polynomial fit equations, (5.25) (5.24) are used such that $|V|_{\text {Difference }}$ and $\phi_{\text {System }}$ can be calculated from $|V|_{\text {Measured }}$ and $\phi_{\text {Measured }}$, Fig. 5.31 and Fig. 5.32, 


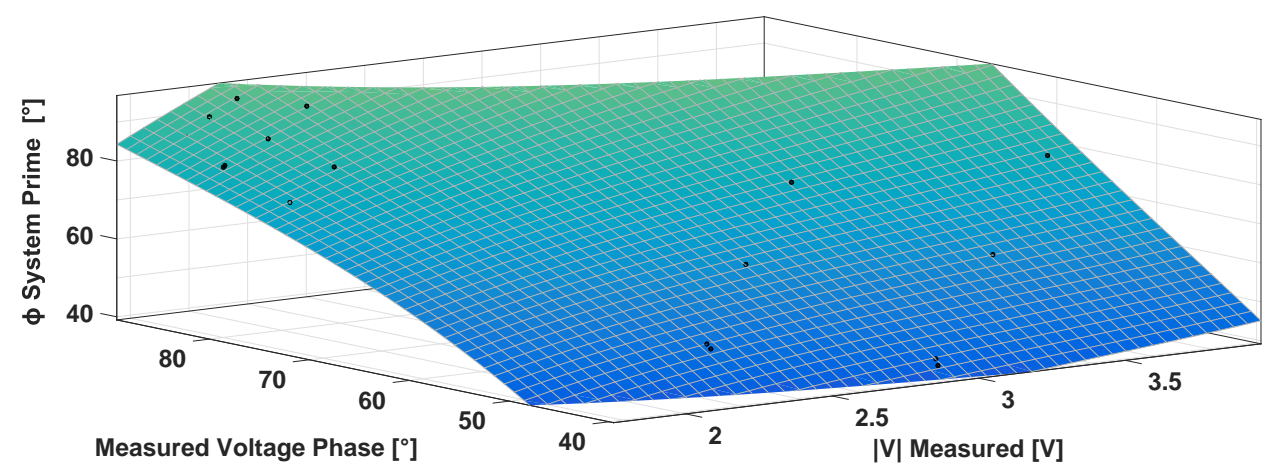

Figure 5.32: Measured Voltage Magnitude $|V|_{\text {Measured }}$ vs Measured Voltage Phase $\phi_{\text {Measured }}$ vs $\phi_{\text {System'. }}$

$$
\begin{aligned}
\phi_{\text {SystemCalc }}{ }^{\prime} & =79.82+\left(10.1 *|V|_{\text {Curve }}\right)+\left(21.88 * \phi_{\text {Curve }}\right)+\left(-1.394 *|V|_{\text {Curve }}{ }^{2}\right) \\
& +\left(4.942 *|V|_{\text {Curve }} * \phi_{\text {Curve }}\right)+\left(-1.106 * \phi_{\text {Curve }}{ }^{2}\right)+\left(0.3014 *|V|_{\text {Curve }}{ }^{3}\right) \\
& +\left(0.02437 *|V|_{\text {Curve }}{ }^{2} * \phi_{\text {Curve }}\right)+\left(1.612 *|V|_{\text {Curve }} * \phi_{\text {Curve }}{ }^{2}\right)+\left(0.7769 * \phi_{\text {Curve }}{ }^{3}\right)
\end{aligned}
$$

$$
\begin{aligned}
|V|_{\text {DifferenceCalc }} & =2.38+\left(0.5885 *|V|_{\text {Curve }}\right)+\left(687.4 E^{-6} * \phi_{\text {Curve }}\right)+\left(247.8 E^{-6} *|V|_{\text {Curve }^{2}}\right) \\
& +\left(698.4 E^{-6} *|V|_{\text {Curve }} * \phi_{\text {Curve }}\right)+\left(5396 E^{-6} * \phi_{\text {Curve }} * \phi_{\text {Curve }^{2}}{ }^{2}\right) \\
& +\left(-2.63 E^{-5} *|V|_{\text {Curve }}{ }^{3}\right)+\left(361.4 E^{-6} *|V|_{\text {Curve }}{ }^{2} * \phi_{\text {Curve }}\right) \\
& +\left(326.9 E^{-6} *|V|_{\text {Curve }} * \phi_{\text {Curve }^{2}}\right)+\left(536.3 E^{-6} * \phi_{\text {Curve }^{2}}\right)
\end{aligned}
$$

where

$$
\begin{aligned}
|V|_{\text {Curve }} & =\frac{|V|_{\text {Measured }}-2.384}{0.588} \\
\phi_{\text {Curve }} & =\frac{\phi_{\text {Measured }}-69.33}{16.58}
\end{aligned}
$$

This results in the calculation of the calibrated sensor voltage magnitude and phase (5.27) and (5.28). 


$$
\begin{gathered}
|V|_{\text {Cal }}^{\prime}=|\mathrm{V}|_{\text {Measured }}-|\mathrm{V}|_{\text {DifferenceCalc }} \\
\phi_{\text {Cal }}{ }^{\prime}=\phi_{\text {Measured }}-\phi_{\text {SystemCalc }}
\end{gathered}
$$

The average errors across the calibration range are $0.2139 \Omega$ and $0.2263^{\circ}$ for the impedance magnitude and phase respectively. The $\mathrm{R}^{2}$ values are 0.9997 and 0.9976 for the second calibration method's impedance magnitude and phase respectively, Fig. 5.26. and Fig. 5.27k . It can be seen that the second method is slightly less accurate in producing calibrated values however, it is less computational intensive thus faster to implement. For the in-vivo experiment described in this thesis, the first calibration method was used as all data processing was done offline thus computation complexity was not an issue and data accuracy was of great importance however, for on-chip calibration implementation the second method should be used. Conversion to admittance and then to a volume is not discussed in this section and will be discussed in the following chapter.

\section{Calibration Methods Calculation Summary}

A summary of each calibration method is shown below. Both the inital generation of calibration curves along with how to calibrate and calculate any measured load.

\section{Calibration Method 1:}

Generation of calibration curves:

1. Generate transformation coefficients $a, b, c, d$

2. Calculate $\mathrm{R}_{\text {Calc }}, \mathrm{C}_{\text {Calc }}$, Ideal sensor voltage $|V|_{\text {Ideal }} \phi_{\text {Ideal }}, \phi_{\text {System }},|V|_{\text {Difference }}$ at each calibration point

3. Generate polynomial fit for:

(a) $\mathrm{R}_{\text {Calc }}$ vs $\mathrm{C}_{\text {Calc }}$ vs $\phi_{\text {System }}$ 
(b) $\mathrm{R}_{\text {Calc }}$ vs $\mathrm{C}_{\text {Calc }}$ vs $|V|_{\text {Difference }}$

Find calibrated impedance, $|Z|_{\mathrm{Cal}}$ and $\phi_{\mathrm{ZCal}}$, at any value after initial calibration curves generated:

1. Calculate $I_{\text {Calc }}, Q_{\text {Calc }}$, with transformation coefficients a, b, c, d

2. Solve for $\mathrm{R}_{\mathrm{Calc}}, \mathrm{C}_{\mathrm{Calc}}$ using known frequency, current, $\mathrm{I}_{\mathrm{Calc}}$, and $\mathrm{Q}_{\mathrm{Calc}}$

3. Plug $\mathrm{R}_{\mathrm{Calc}}, \mathrm{C}_{\mathrm{Calc}}$ into polynomial fit equations to solve for $\phi_{\text {SystemCalc }}$ and $|V|_{\text {DifferenceCalc }}$

4. Calculate calibrated sensor voltage $|V|_{\text {Cal }}$ and $\phi_{\text {Cal }}$

5. Calculate calibrated load impedance $|Z|_{\mathrm{Cal}}$ and $\phi_{\mathrm{ZCal}}$

\section{Calibration Method 2}

Generation of calibration curves:

1. Calculate ideal voltages $\left(|V|_{\text {Ideal }}, \phi_{\text {Ideal }}\right)$ and measured voltages $\left(|V|_{\text {Measured }} \phi_{\text {Measured }}\right)$ at each calibration point

2. Calculate $|V|_{\text {Difference, }}$ and $\phi_{\text {System }}$ ' using ideal and measured voltages

3. Generate polynomial fit for:

(a) $|V|_{\text {Measured Vs }} \phi_{\text {Measured Vs }}|V|_{\text {Difference' }}$

(b) $|V|_{\text {Measured }}$ Vs $\phi_{\text {Measured }}$ Vs $\phi_{\text {System }}$

Find calibrated impedance, $|Z|_{\mathrm{Cal}}$, and $\phi_{\mathrm{zCal}}{ }^{\prime}$, at any value after initial calibration curves generated:

1. Calculate $|V|_{\text {Measured }}$ and $\phi_{\text {Measured }}$ using the output voltages of the volume module $\mathrm{I}_{\text {Meas }}$ and $\mathrm{Q}_{\text {Meas }}$.

2. Plug $|V|_{\text {Measured }}$ and $\phi_{\text {Measured }}$ into polynomial fit equations to calculate $|V|_{\text {DifferenceCalc' }}$ and $\phi_{\text {Systemcalc }}$ 
3. Calculate calibrated voltages $|V|_{\mathrm{Cal}}$, and $\phi_{\mathrm{Cal}}{ }^{\prime}$

4. Calculate calibrated impedance $|Z|_{\mathrm{Cal}}$ and $\phi_{\mathrm{ZCal}}{ }^{\prime}$

\subsection{Dynamic PV Loop Evaluation}

Prior to the in-vivo study the PV system must be evaluated dynamically to test various aspects of the system including battery life, pressure volume loops, control architecture, etc. Static testing is relatively easy to achieve, for example, static pressure tests can be completed by placing the catheter inside a sealed tube and creating a vacuum. Static volume tests are also relatively simple to complete. Typically a cuvette containing salt water with a set volume is used. Here, the catheter is placed in the cuvette and the corresponding output values from the PV system are measured. Combining both pressure and volume as well as making them change at the same time is more difficult. A pneumatic system is used to achieve this. Designed in-house by Transonic Scisense, this "Volume Pulser' is designed to change its pressure and volume at a set frequency, Fig. 5.33. Similar professional solutions exist, [269], however, they are quite costly, thus not used here.

Due to design constraints the "Volume Pulser" is only able to emulate very low heart rates and medium sized volumes, however, it is still able to generate proper cycles to measure PV loops. The catheter, attached to the PV system, is inserted into the fluid filled volume chamber where the catheter is able to measure the change in pressure and volume. The PV system is typically wired into the development module to allow access to the power supply and additional testing signals. The measured data is sent to the Rx Unit, Section 4.8, where the data is received and processed. The processing and viewing software developed in LabView will be discussed in the following chapter.

Battery life testing is performed using this setup and the implant is placed in a combination of the three modes described in Section 4.7.2. Various tests were run to confirm the current consumption results in Section 4.7.3. For example, the implant was placed in duty cycle mode 


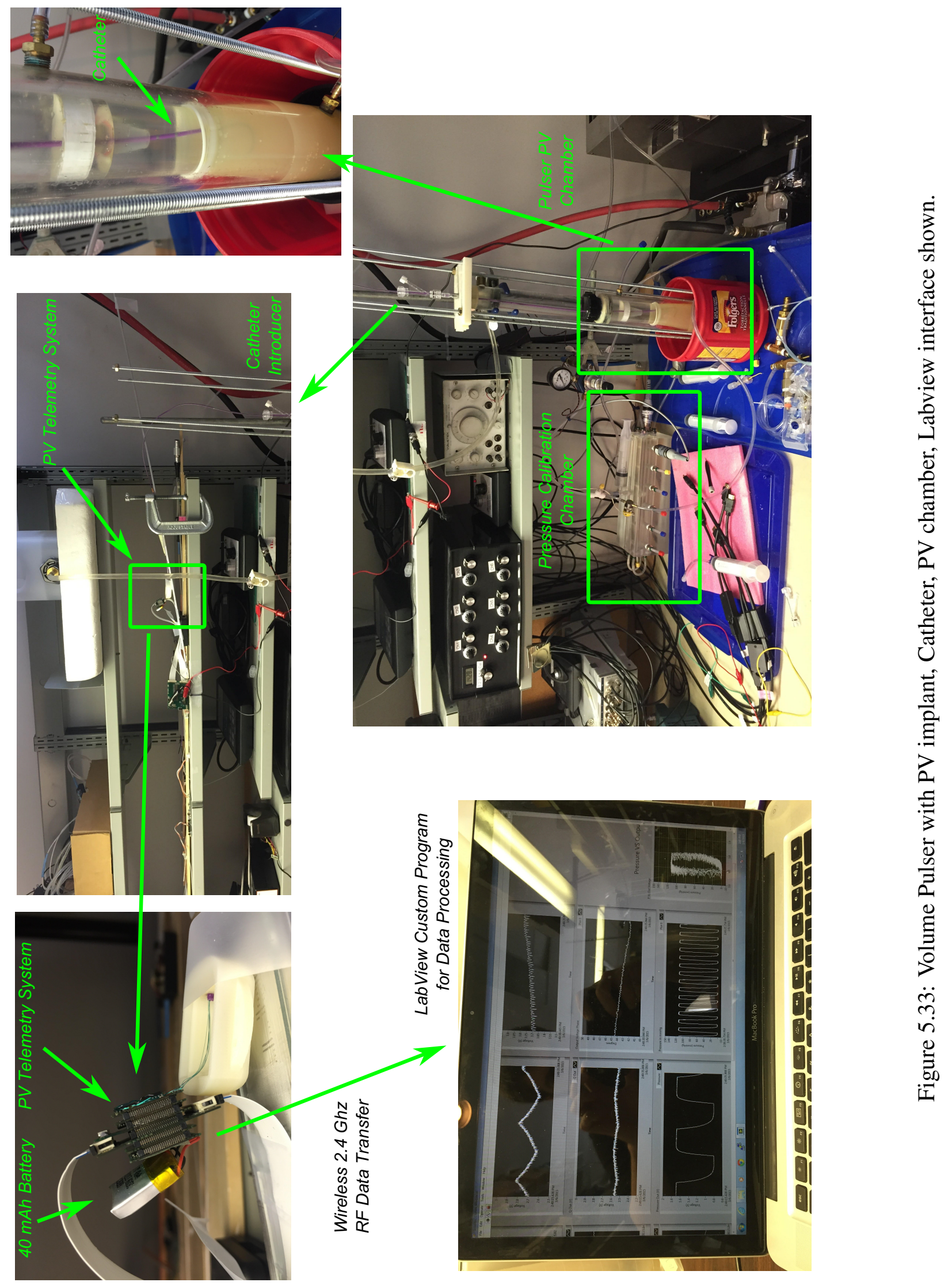




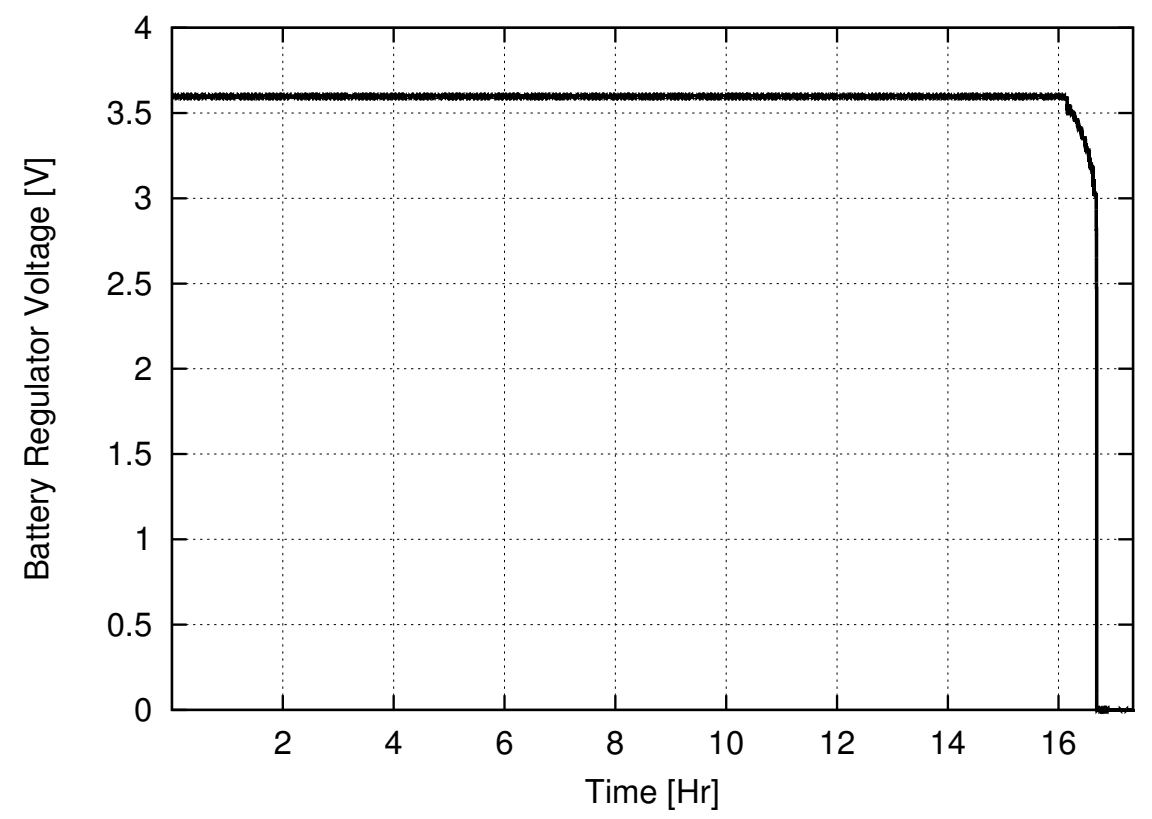

Figure 5.34: Measured battery life with a duty cycle of 2 min on and 2 min with various Rx cycles.

operating on a 2 min on, 2 min off cycle with additional Rx cycles mixed in, lasting approximately 16.5 hours, Fig. 5.34. An additional longer test with a different duty cycle is also shown, Fig. 5.35. Here, the battery lasted approximately 58 hours. These tests were repeated with different measurement intervals along with different RF power settings to give an outline of the typical battery life.

Along with battery life, the control architecture is also tested at this stage. Various aspects of the system are tested including timing, data operation and validity, implant range, etc. In addition, the implant is tested inside its enclosure to ensure no data loss occurs. Testing in the "Volume Pulser" is the final stage before implanting the system inside a live animal. 


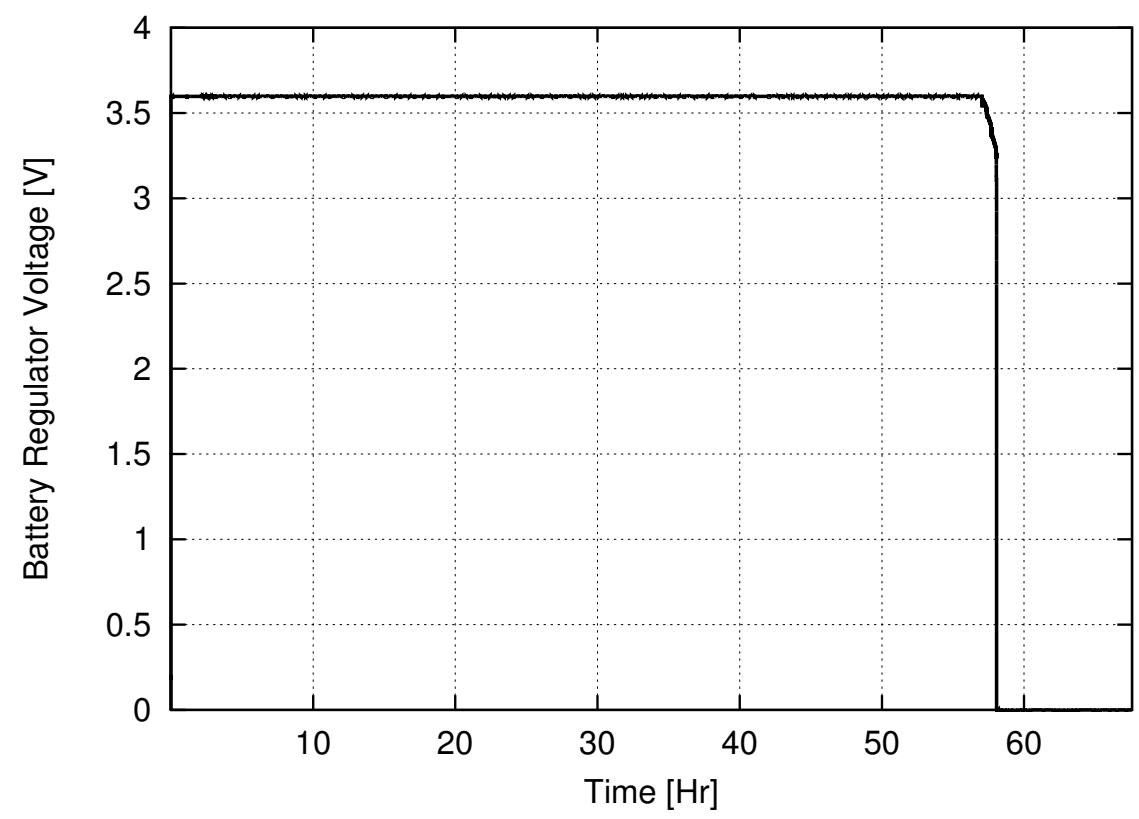

Figure 5.35: Battery life of implant in an extended duty cycle.

\subsection{Conclusion}

In this chapter the in-vitro characterization of the implantable telemetry system is presented. The outcome of the phantom tissue study [257] study helped to determine the minimum RF output power that the transmitter $\left(\mathrm{T}_{x}\right)$ needs to provide to still receive data from the implant while having minimum power consumption in a typical medical measurement environment, i.e., instrument noise, non ideal antenna matching, spectrum interference, etc.

A custom implant capsule was designed and printed with four materials ( 3 plastic, 1 metal). The implants were evaluated by a 98 day and 1 week water immersion study. Although minor moisture occurred, ABS-M30i is chosen for future implant capsules as to ensure biocompatibility, high strength and precision when compared to ABS and RDG720. The stainless steel capsule provided the highest precision among the four materials however, additional postprocessing time is required. Further studies are required to determine the cause of membrane reactions and the exact failure point of the ABS-M30i package.

Calibration of the pressure and volume electronics were presented. The MEMS absolute 
pressure sensor was calibrated inside a fluid-filled pressure chamber that allows for variations in temperature and pressure. Four point calibration was used to calibrate for the variation of the wheatstone bridge offset over temperature and variation of the bridge gain over temperature thereby providing $1^{s t}$ order temperature compensation. All calibration data is stored within the pressure IC at two temperatures, $22.8^{\circ} \mathrm{C}$ and $43.2^{\circ} \mathrm{C}$ representing a wide range of temperature operation. The sensor was calibrated over a $450 \mathrm{mmHg}$ pressure range, from $-135 \mathrm{mmHg}$ to $315 \mathrm{mmHg}$.

Two calibration methods for the volume electronics were presented. The average errors across the calibration range for the first calibration method are $0.211 \Omega$ and $0.2^{\circ}$ for the impedance magnitude and phase respectively. The average errors across the calibration range for the second calibration method are $0.2139 \Omega$ and $0.2263^{\circ}$ for the impedance magnitude and phase respectively. The first calibration method is used in the in-vivo study.

Dynamic PV loop evaluation was provided through the use of a custom designed system, "Volume Pulser". Here, battery life, dynamic PV loops and control architecture were tested. This is the final stage of testing before implanting inside of a live animal subject. 


\section{Chapter 6}

\section{In-Vivo Implant Characterization *}

In this chapter, details of the in-vivo study are presented. The PV telemetry system designed in Chapter 4 was evaluated in a $65 \mathrm{~kg}$ swine (pig). The implant study was performed by a trained group of surgeons at Western University Veterinary Services in accordance with the approved ethics protocol AUP Number: 2014-003. The study was designed to evaluate the PV system in a non-anaesthetised test subject, i.e., freely moving animal. In addition, the study was completed to determine initial catheter-heart interaction. Data was collected via the RF link from the implant to an external laptop. The implant is designed to transmit raw voltages, thus additional data processing is completed at the receiver side to process the data into calibrated pressures and volumes.

This chapter is separated into two major sections. First, design details of the custom measurement application are presented in Section 6.1 followed by a comprehensive analysis of the in-vivo study in Section 6.2 .

\subsection{LabView Custom Measurement Application}

In Chapter 4 details of the wireless receiver unit were discussed with a focus on the hardware development. The initial software solution for data viewing was presented, however, the soft-

*Portions of this chapter are published in BIOCAS 2015 [270] and Spring Biomedical Devices journal [240]. 


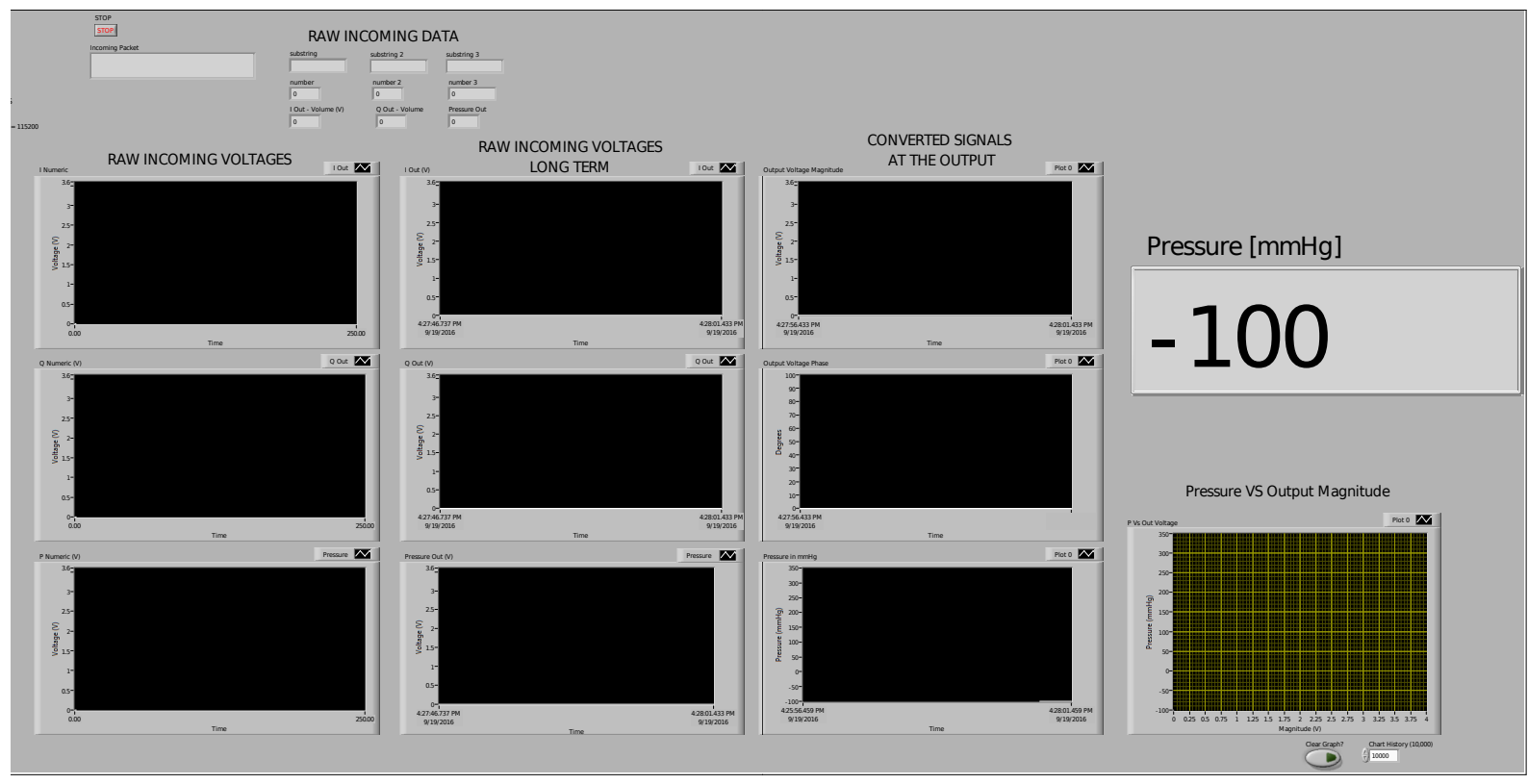

Figure 6.1: Custom application front end: Receive data interface.

ware was only able to display the raw I, Q, and P values and did not include any additional processing, calibration, or graphing capabilities. In addition, viewing the raw data in a serial communication window is not ideal in a surgical environment. As traditional pressure-volume (PV) measurement systems are directly tethered to their end communication boxes, real-time data can be viewed and captured with no impact on battery life or signal quality. In implantable devices sending a constant stream of data to display will have a great impact on battery life. Nevertheless, the data displayed is of great importance to the operating surgeons who may have to adapt their movements to the incoming data.

To overcome these challenges, a custom application is created in National Instruments LabView to further process, log, and display the implant data, Fig. 6.1. Furthermore, it is also important to send information back to the implant in order to change various aspects of the control system including transmission power, acquisition mode, etc, Fig. 6.2. The following subsections describe the serial protocol setup, reading data transferred from the implant, writing data to the implant, and finally some limitations with the initial version of software. 


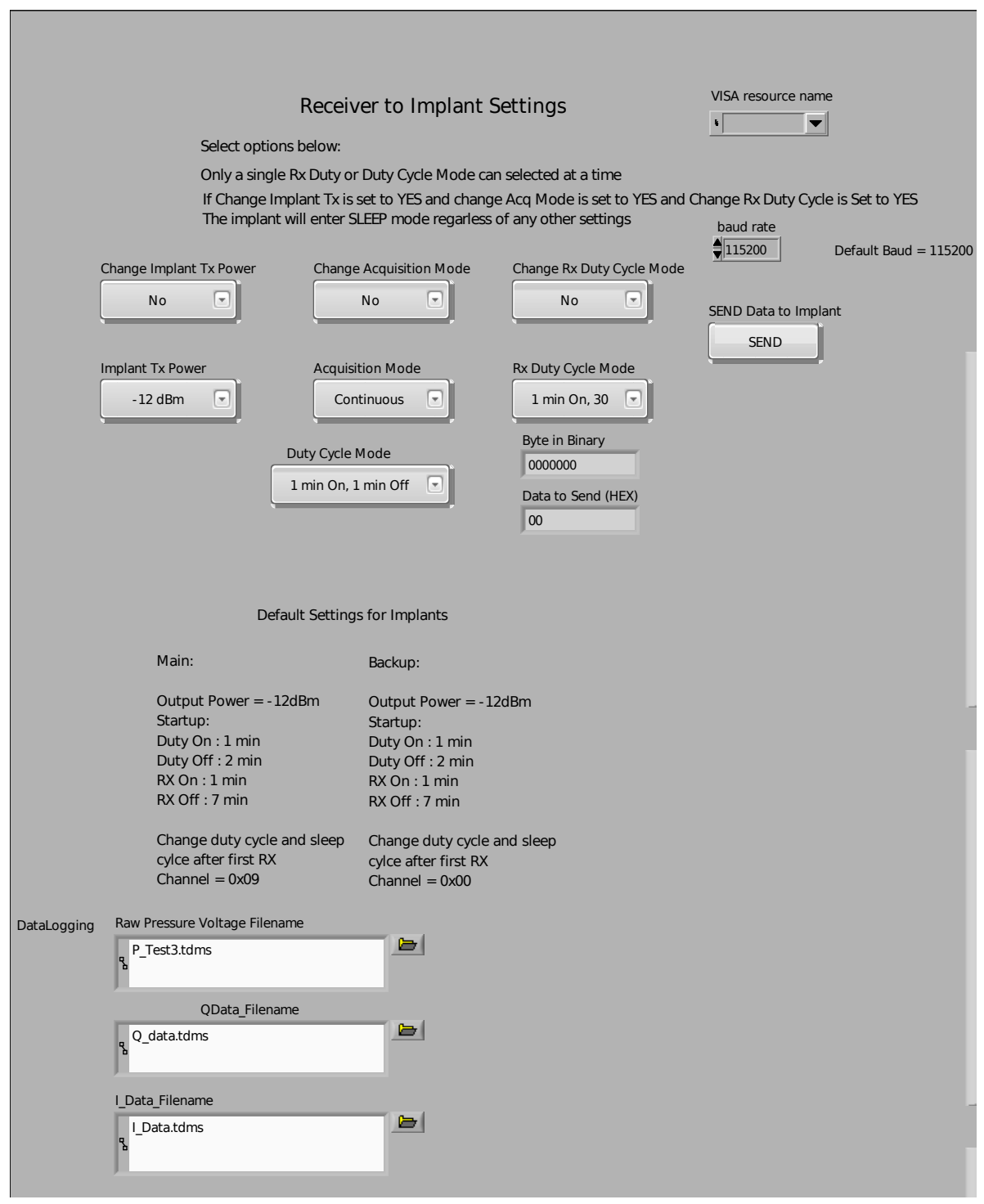

Figure 6.2: Custom application front end: Send data interface. Application can change Tx power, acquisition modes, receive and duty cycle times using a single byte transmission. 


\subsubsection{Serial Port Setup}

All serial port settings are setup through the graphical user interface (GUI) of the application. Rather than hardcode these into the application, it is more appropriate to have these settings change by the user. The main information that the user must provide to receive data are the baud rate and the COM port used. This allows different hardware receivers with different baud rates to be used. In addition, the COM port will change based on which USB port is used, thus it is important the user be able to change the COM port used. The serial port is setup to receive data at a baud rate of 115200 with no hardware handshaking. If the setup fails or the receiver is disconnected at any point during the programs opperation, an error flag is presented asking the user to reconnect or check the serial settings.

An array is used to buffer the incoming data before it is processed by the internal program. This is to ensure any delays caused by the graphing or data logging in the internal program will not cause dropped packets or a loss of information. All data will be received unless the user chooses to send information to the implant via the send button.

\subsubsection{Data Read}

The incoming data from the wireless receive unit is processed in five steps: 1) string splitting, 2) covert integers to voltages, 3) log data, 4) convert to real and imaginary voltages, ) and graphing end data, Fig. 6.3

Recall from Chapter 4, that the incoming packet format is IIIIQQQQPPPP (12 bytes), this incoming string must be first separated into individual I, Q and P values. A separate VI (LabView sub-program) is used to complete this function. Offsets are used to separate each 4 byte ASCII representation of I, Q, P.

Following string splitting, each string is converted into their corresponding integer value. Each integer represents a register value from the ADC and thus must be converted into a voltage 


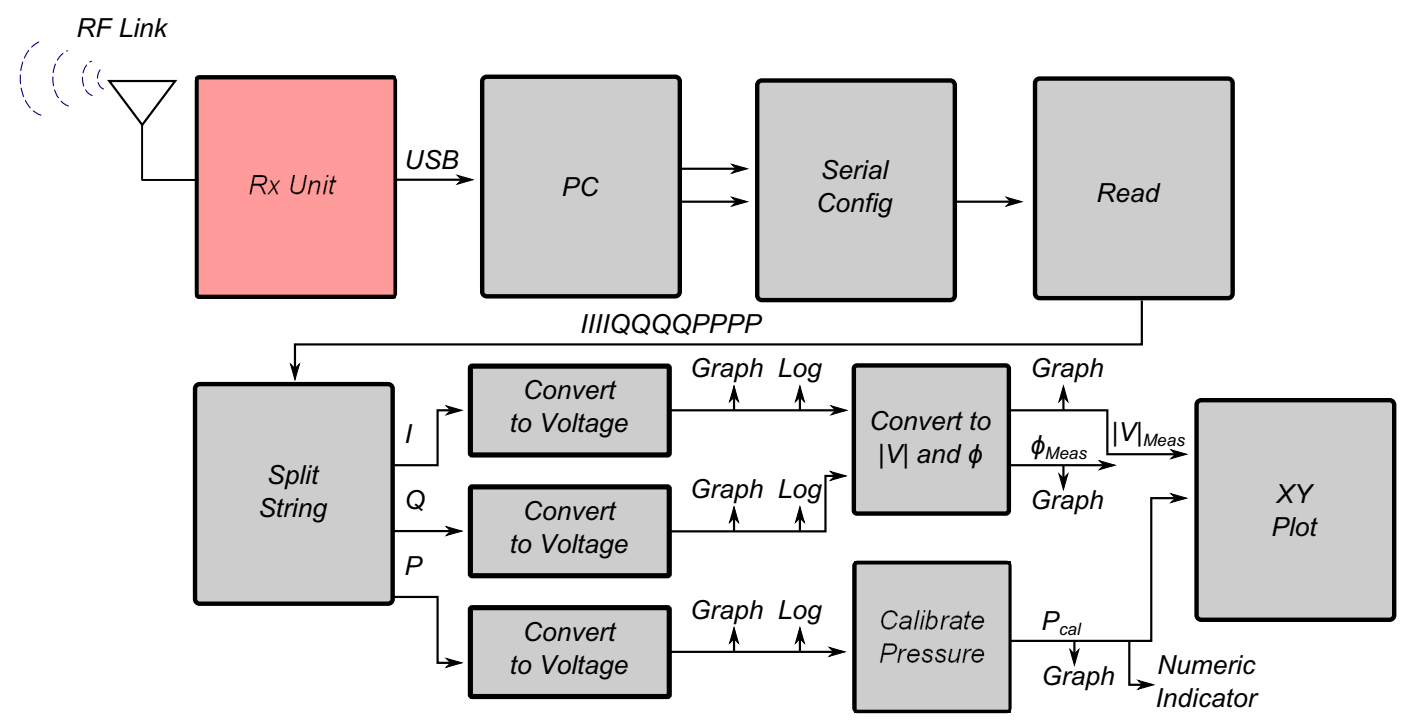

Figure 6.3: LabView application block diagram including sub-blocks: Rx Unit, serial config, read, split string, convert to voltage, convert to $|Z|_{\text {Meas }}$ and $\phi_{\text {Meas }}$, calibrate pressure, and XY plot.

using 6.1).

$$
\text { Voltage }=\frac{\text { Input }}{1023 / 3.6 \mathrm{~V}}
$$

At this stage, the raw $\mathrm{I}_{\text {Meas }}, \mathrm{Q}_{\text {Meas }}$, and pressure voltages are graphed in both short and long term graphs, Fig. 6.1. The graphs are useful, in that they enable the user to ensure that there is incoming data, however, they do not indicate any true physiological values at this point, due to lack of calibration, Section 5.3.1 and 5.3.2. Major failures or data inconsistances can be detected at this point. For example, if both I and Q values are railed at 3.6 V there is most likely an issue with the implant. Similarly, if both are grounded there also may be an issue. Viewing both the volume output channels $\mathrm{I}_{\text {Meas }}, \mathrm{Q}_{\text {Meas }}$ and Pressure output channel (voltage) can also indicate possible failures, as the measured data is from the heart and therefore periodic, all channels must match the same frequency or heart rate. If one or more channels is incorrect then most likely a failure has occurred.

All raw data is saved to disk at this point in the application before any additional changes are made to the raw data. This allows the data to be modified and fully calibrated after the experiment is complete. In initial implementations of this application, the data was saved to disk 
in Microsoft Excel format. This allowed for ease of use when reading back the information and simple processing. However, the speed of the program was greatly impacted. The delay was due to the opening and closing of Excel each time a data point is saved, rather than the application itself. Furthermore, if Excel is used, the update delay will increase over time impacting if the user needs to react to the changing signal. For example, when Excel data logging was used the delay increased to minutes after a five minute period of data logging. Therefore, it is reccommended to use Excel data logging only for very slow signals where signals do not need to be reacted to. Alternatively, LabView's proprietary format, TDMS is used. The main reason to use the TDMS format over the Excel format is that a TDMS file can be left open throughout the whole data logging process. The files are created when the application begins and all write operations are done on these files without opening or closing the main file. The files are all closed when the application is ended by the stop button. Another advantage is that if there is a connection failure midway through the data logging process, all data is still saved based on the TDMS format architecture. Date and time information is saved as header information in the file as well. A disadvantage of using this format is that it requires a special plugin from National Instruments in order to read the files in Excel. Once opened, each file can be saved in any format (CSV, Excel, Txt, etc) required by the user.

At this stage, $\mathrm{I}_{\text {Meas }}, \mathrm{Q}_{\text {Meas }}$ are converted to $|Z|_{\text {Meas }}$ and $\phi_{\text {Meas }}$ for display purposes only. They are displayed in graphs for the user to view, Fig. 6.1. Although not calibrated, the measured signals will display patterns in the measured signal including phase increases. For example, the phase will greatly increase if the catheter is close to the left ventricle wall regardless if the system is fully calibrated. The pressure calibration (6.2) is hard coded into the application as it calibrated separately and not computationally intensive unlike the volume calibration.

$$
\text { Pressure }=\frac{\text { Voltage Output }}{8 \mathrm{mV} / \mathrm{mmHg}}-135 \mathrm{mmHg}
$$

The calibrated pressure is displayed in both a graph and in a large numeric indicator, Fig. 6.1 
The numeric indicator's purpose is to provide a straightforward view of the pressure value in $m m H g$. This value will indicate to the surgeon if they have guided the catheter into the LV, based on the expected pressures..

The calibrated pressure signal is plotted against $|Z|_{\text {Meas }}$ to create a loop, Fig. 6.1. Although, this is not a pressure vs volume loop, the size and shape are still useful information for surgeon. The volume and admittance are not shown in this version of the application as calibration is done offline (after the experiment). Furthermore, this version's main use is to indicate the implant is operational and that the catheter has entered the LV. Data quality and accuracy of the system is determined after the calibration and data analysis are completed.

\subsubsection{Remote Implant Data Write}

The LabView application also provides the ability to send commands back to the implant to change various aspects of the system including three main categories: transmission power, acquisition mode, and receive mode. The user selects which commands are to be sent from the application GUI, Fig. 6.2. The user is able to select if any of the three main categories should be changed in the implant, each with its own sub-settings to send to the implant. The commands are encoded into a single byte packet in order to conserve battery and limit the amount of transmissions required. Thus, only a limited amount of combinations and commands can be sent. In some cases, bits will be multiplexed, more on this below. Transmissions can be sent at any time during the program by configuring the drop down menus and hitting the send button on the application. However, they will only be received when the implant is switched into receive mode, i.e., implant transceiver is in Rx. Described in Section 4.7, the implant will enter receive mode at set time intervals determined by the user before implantation. As a result, it is very important to keep track of the time when the implant will enter receive mode for the first and subsequent times.

The packet format is $\mathrm{X}_{7} \mathrm{X}_{6} \mathrm{X}_{5} \mathrm{X}_{4} \mathrm{X}_{3} \mathrm{X}_{2} \mathrm{X}_{1} \mathrm{X}_{0}$ with each bit's function described in Table 6.1

The acquisition mode type is determined by the bit $\mathrm{X}_{0}$. If this bit is 0 the implant will enter 
Table 6.1: Rx to Implant Packet Format

\begin{tabular}{l|l}
\hline Bits & Function \\
\hline $\mathrm{X}_{0}$ & Acquisition Mode Type \\
\hline $\mathrm{X}_{2} \mathrm{X}_{1}$ & $\begin{array}{l}\text { if } \mathrm{X}_{0}=1-\text { Duty cycle mode times } \\
\text { if } \mathrm{X}_{0}=0-\text { Rx duty cycle times }\end{array}$ \\
\hline $\mathrm{X}_{3}$ & Change implant Tx power \\
\hline $\mathrm{X}_{4}$ & Change Rx Duty Cycle \\
\hline $\mathrm{X}_{5}$ & Change Acquisition Mode \\
\hline $\mathrm{X}_{7} \mathrm{X}_{6}$ & Implant Tx Power Level \\
\hline
\end{tabular}

continuous mode, i.e., sample and transmit continuously until a set Rx timer, or if 1 the implant will enter duty cycle mode. Bits $\mathrm{X}_{4}$ and $\mathrm{X}_{5}$ are used as control switches to change Rx Duty Cycle and Acquisition Mode, respectively. These bits must be 1 in order for $\mathrm{X}_{0}$ and $\mathrm{X}_{2} \mathrm{X}$ to be valid.

Bits $\mathrm{X}_{2} \mathrm{X}_{1}$ are used to control the specific time intervals (on and off) in the Rx and Duty Cycle modes. Rather than only four time options available (2 bit) and used for both $\mathrm{Rx}$ and Duty Cycle modes, the bits are multiplexed to allow for different options for each with only 2 bits. A combinational logic switch is used to ensure only 1 mode is valid at a time and if both (change Rx and change Duty Cycle) are set to high, Rx duty cycle changes will take priority, Fig. 6.4. The truth table is shown in Table 6.2. If both modes required to be changed, two transmissions are required. The LabView application does not directly send timing information as it only sends a 2 bit combination that the implant will decode into a specific on and off time. These on and off times must be hard corded into the implant before use. A wide variety of on and off times are chosen for the in-vivo study, Table 6.3, providing the user with flexibility in acquisition modes.

The implant transmission power is changed when $\mathrm{X}_{3}$ is set to 1 . Bits $\mathrm{X}_{7} \mathrm{X}_{6}$ are used to control the specific Tx output power level used. Power levels are chosen based on the results of the phantom tissue study, Section 5.1.3, and range from a minimum of $-12 \mathrm{dBm}$ to a maximum of $1 \mathrm{dBm}$. As with the Rx and Duty cycle modes, the Tx output power levels are hardcoded into the implant's control code before implantation. 


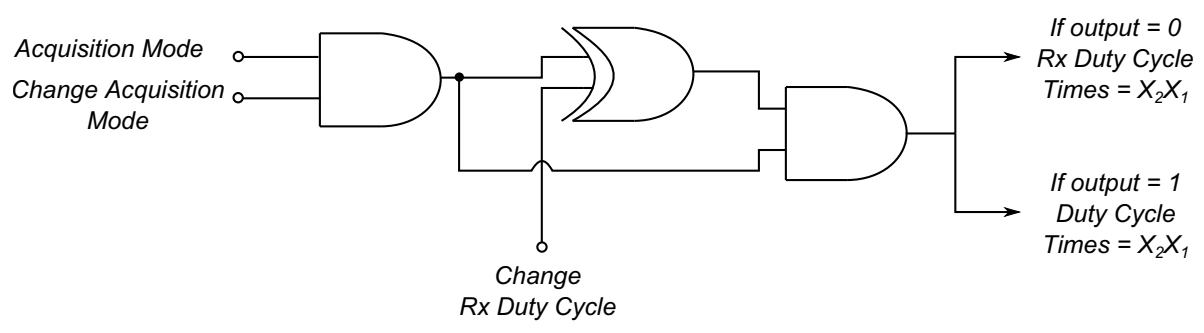

Figure 6.4: Combinational logic used such $\mathrm{Rx}$ changes take precedence over Duty cycle changes for bits $\mathrm{X}_{2} \mathrm{X}_{1}$.

Table 6.2: Combinational Logic Truth Table

\begin{tabular}{l|l|l|l}
\hline Acquisition Mode & Change Acquisition Mode & Change Rx Duty Cycle & Output \\
\hline 0 & 0 & 0 & 0 \\
\hline 0 & 0 & 1 & 0 \\
\hline 0 & 1 & 0 & 0 \\
\hline 0 & 1 & 1 & 0 \\
\hline 1 & 0 & 0 & 0 \\
\hline 1 & 0 & 1 & 0 \\
\hline 1 & 1 & 0 & 1 \\
\hline & 1 & 1 & 0 \\
\hline
\end{tabular}

Table 6.3: Duty Cycle and Rx On and Off times used in in-vivo experiment

\begin{tabular}{l|l|l}
\hline Bits & Duty Times & Rx Times \\
\hline 00 & 1 min Sampling, 9 min off & 1 min on in Rx, 30 min off \\
\hline 01 & $30 \mathrm{sec}$ Sampling, 9.5 min off & 2 on, 1 hr off \\
\hline 10 & 5 min Sampling, 5 min off & 5 min on, 2 hr off \\
\hline 11 & 1 min Sampling, 59 min off & $30 \mathrm{sec}$ on, 2 min off \\
\hline
\end{tabular}


When the implant receives this data, the packet is decoded and each option is processed separately. The implant will remain in receive mode until the Rx timer expires, enabling multiple commands to be sent and received from the external unit. This implementation of the custom LabView application is used in the in-vivo study.

\subsubsection{Limitations of Application V1}

This initial application, although much improved over the basic raw data viewer, still has several limitations. First, as all the calibration is completed offline, there is no built in calibration into the program. This prevents real time calibrated admittance and volume to be displayed. Furthermore, true PV loops cannot be displayed without the volume being converted in real time. A major limitation of using LabView to create a custom application is its lack of resolution scaling. It is very difficult to view all graphical data, implant settings and file settings all in a single window, hence, the requirement of tabbed windows. Moreover, additional graphs and data logging points should be added to provide the user with more beneficial information. In terms of receive unit to implant communications, it is not ideal for the user/surgeon to know ahead of time the possible measurement cycles or power levels required. Thus, it would be better to directly send the value rather than selection flags, i.e sending the register value for $1 \mathrm{dBm}$. However, by using this method, the Rx unit to implant packet size would increase.

\subsection{In-Vivo Study}

The PV system designed in Chapter 4 and characterized in Chapter 5 is evaluated in-vivo using a swine also known as a pig. An initial experiment involving a pig was completed, however it was unsuccessful due to medical complications with the animal unrelated to the experiment. The animal was euthanized in accordance to the ethics protocol. All procedures and results of described in this chapter refer to the second in-vivo implantation with a similar sized pig.

This section presents all aspects of the study including implant preparation, data collection, 
tissue analysis, etc. A detailed discussion is provided explaining the issues encountered in the study along with future recommendations. A comparison to previous published works is also provided in the discussion.

\subsubsection{Experiment Overview}

The implant study was performed at Western University Veterinary Services by a trained surgical and technical team. This team consisted of Filip Konecny (Transonic Scisense Inc.), Alexander El-Warrak (Veterinary Services, Western University), Heather Cadieux-Pitre (Veterinary Services, Western University), Tracy Hill (Veterinary Services, Western University), and Chad Hodgson (Transonic Scisense Inc.). All surgical procedures were performed by Filip and Alexander with assistance provided by Heather and Tracy. Additional technical support was provided by Chad Hodgson. The medical procedure and monitoring was performed in accordance with the approved ethics protocol AUP Number: 2014-003. The study was designed to evaluate the PV system in a non-anaesthetised test subject, i.e., freely moving animal. In addition, the study was completed to determine initial catheter-heart interaction.

\subsubsection{Implant Preparation}

To plan for any possible hardware failures, two implants were prepared for the in-vivo surgery. Each implant was identical and calibrated using the process described in Section 5.3. The catheter is inserted into the enclosure and the 7 sensor wires are attached to the top pads on the telemetry system, Fig. 6.5. The catheter opening is sealed inside and out to prevent the catheter from moving when in use. Prior to full encapsulation, the system is tested inside the volume pulser and various sized cuvettes to ensure no hardware faults are observed.

The implant is placed in a custom power up routine where the system will enter duty cycle ( $1 \mathrm{~min}$ on and $2 \mathrm{~min}$ off) mode for $7 \mathrm{~min}$ at which time the implant enters receive mode to receive instructions from the $\mathrm{Rx}$ unit. This routine includes multiple data collection cycles to ensure the implant powers on properly after battery connection and that no hardware faults oc- 


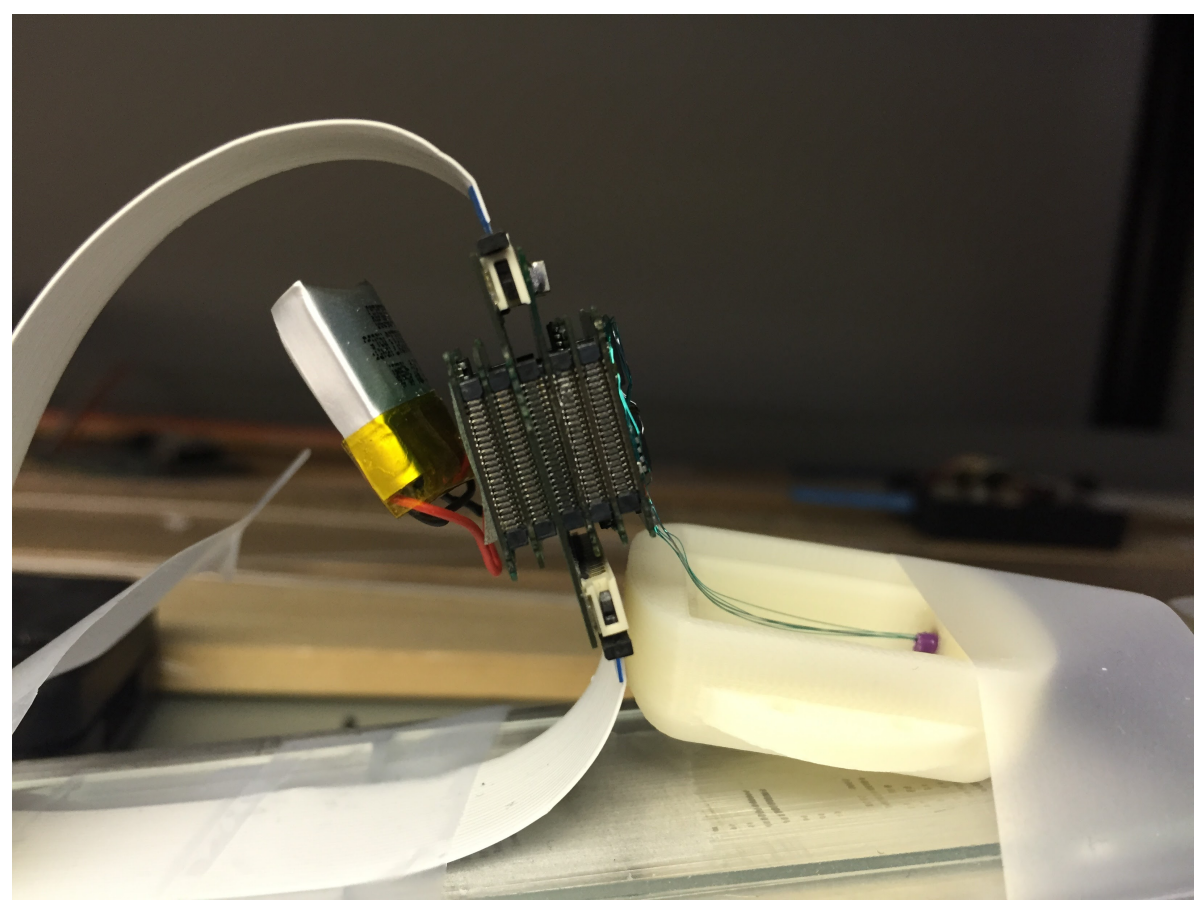

Figure 6.5: Seven sensor wires fed through the catheter into the enclosure attached to the telemetry system.

curred when reassembling the PV system. Entering receive mode during the start up sequence enables the user to choose the measurement cycles at later time rather than hard code them into the control architecture before encapsulation. More importantly, this receive window allows the user to change the $\mathrm{Rx}_{\text {time off }}$ to a longer interval to reduce power consumption, i.e. $1 \mathrm{~h}$, and the user is able to place the implant in sleep mode. This is important as the implant is fully encapsulated typically $24 \mathrm{~h}$ before implantation, the implant must be in sleep state to prevent excessive and unnecessary power loss during non-operational times. An $\mathrm{Rx}_{\text {time off }}$ interval of 1 $\mathrm{h}$ and $\mathrm{Rx}_{\mathrm{time}}$ on $1 \mathrm{~min}$ are used in this experiment while the implant is cured. Both implants are sealed and encapsulated as per the process described in Section 5.2.2 and allowed to cure for $24 \mathrm{~h}$. Once cured the implants are placed in sealed packages for transport, Fig. 6.6.

\subsubsection{Telemetry System Implantation Procedure}

A $65 \mathrm{~kg}$ Yorkshire farm pig was placed under general anesthesia on a dorsal side position. The animal was kept heparinized to achieve an active clotting time of more than 250 seconds. The 


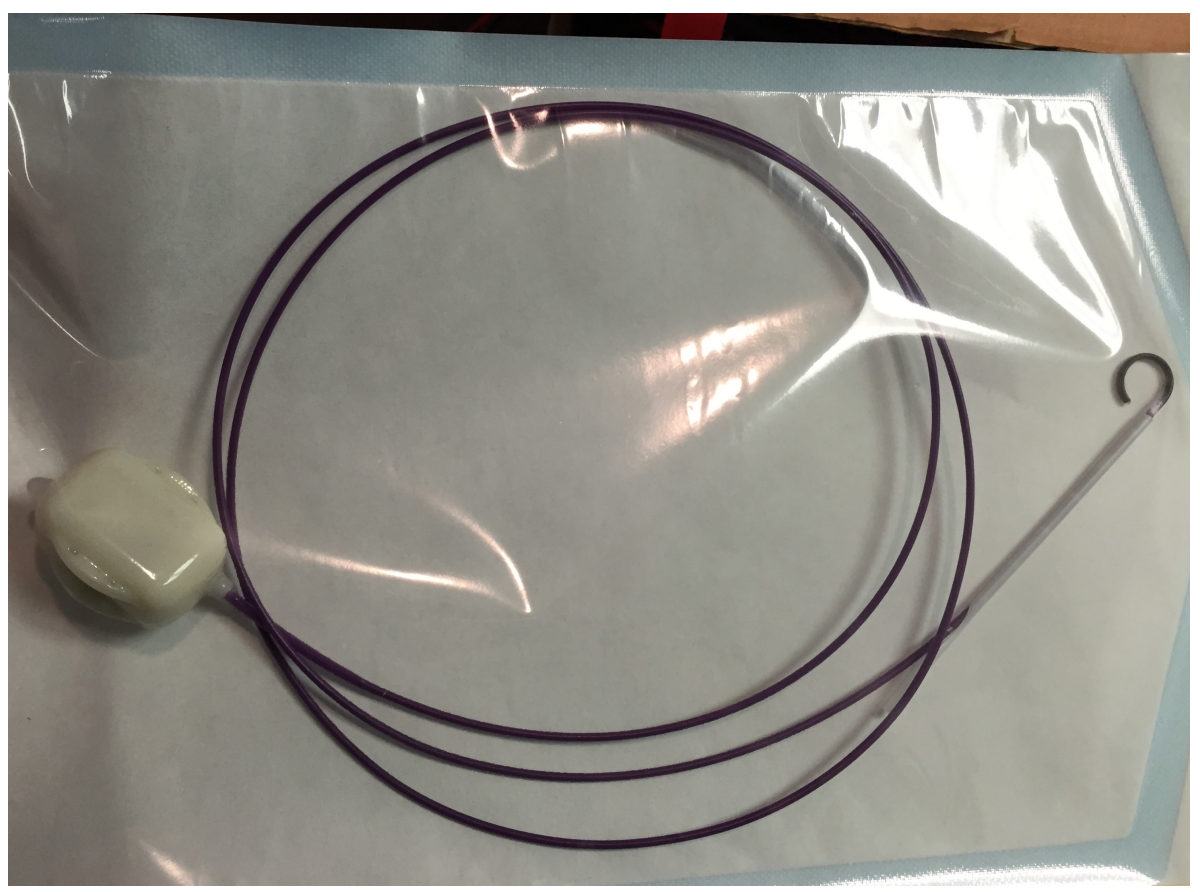

Figure 6.6: PV telemetry system fully enclosed in ABS-M30i package and dipped in silicone. Package used for transport.

right carotid artery (RCA) was exposed through a 7-9 $\mathrm{cm}$ long standard ventral neck surgical approach. The artery was isolated using stay sutures to allow the insertion of a 18-gauge, 2 3/4-inch Seldinger needle. Round tipped 0.038-inch Seldinger guidewire was inserted through the needle's lumen into the RCA. The needle was withdrawn and a guidewire used to direct the ClassicSheath $^{\mathrm{TM}}$ splittable haemostatic introducer (Merit Medical Systems Inc. UT, USA), of the luminal size $7 \mathrm{~F}$ to accommodate the $5 \mathrm{~F}$ catheter. Prior to insertion into the $\mathrm{LV}$, the implant and PV catheter were sterilized with Cidex disinfectant solution (Advanced Sterilization Products, USA). The catheter was secured in place with multiple sutures. The catheter, Fig. 4.4.1, was fluoroscopically guided into the LV via the carotid artery. The position of the catheter sensors was achieved by fluoroscopy and by obtaining an optimal pressure, I, Q values via the RF link, Fig. 6.7. It was noticed on fluoroscopy that the tip of the sensing electrode was fractured. Based on the readings it was decided at this point to secure the catheter to the muscle layer using multiple anchoring sutures to prevent the catheter from exiting the LV. The telemetry system and the remaining excess catheter tubing was coiled and placed in the neck region and 


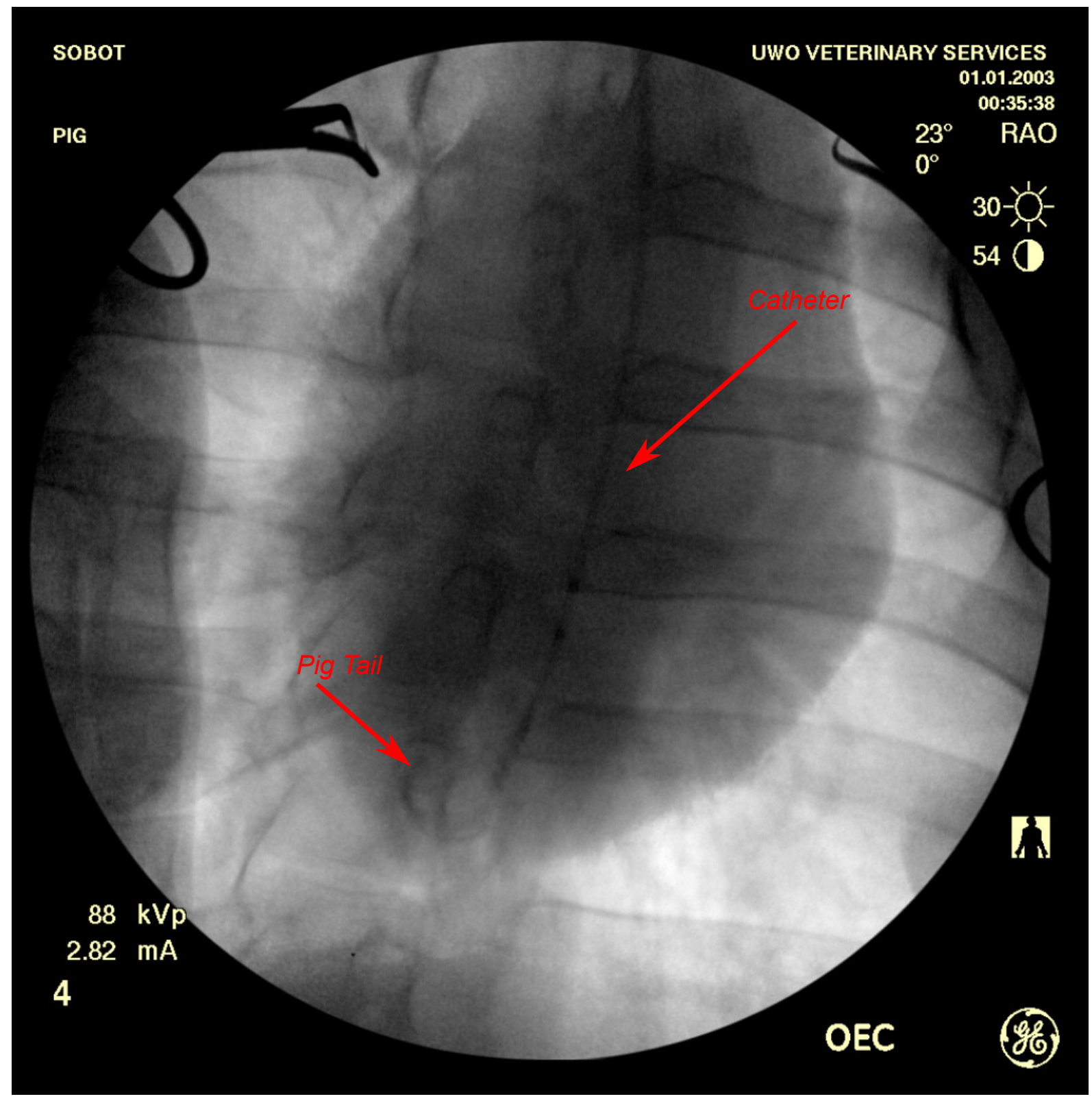

Figure 6.7: Fluoroscope image of 5F PV catheter inserted in the left ventricle. 


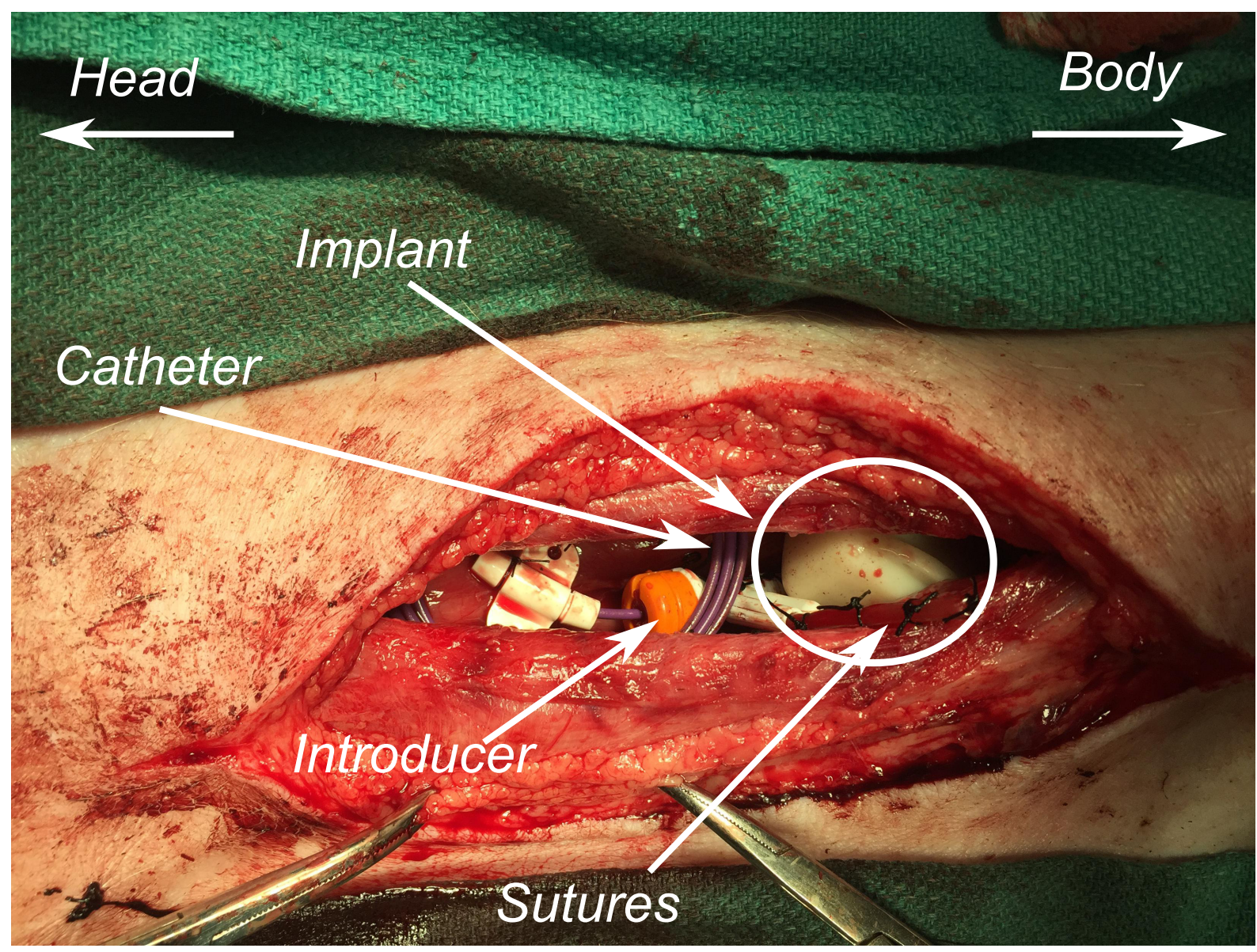

Figure 6.8: PV system implanted in neck region of test subject PV catheter inserted into LV via carotid artery.

anchored with simple interrupted sutures. The surgical site was closed using simple continuous patterns on the muscle and subcutaneous layers using a 3-0 Vicryl, Fig. 6.8, where 3-0 is the size of the suture fiber and Vicryl is the type of suture.

The skin was closed using a subcutilar pattern using 4-0 Vicryl and tissue glue. The final implant location of the implanted telemetry system was confirmed with fluoroscopy, Fig. 6.9.

The neck region was selected for the implant location due to its proximity to the carotid artery. Alternative options were explored which included locating the implant in the backside of the animal with a tunneled catheter to access the carotid artery, however, this can cause unnecessary trauma to the animal and was not used. An alternative approach to access the LV is through the apex of the heart. Open heart surgery is required for this method and is preferred 


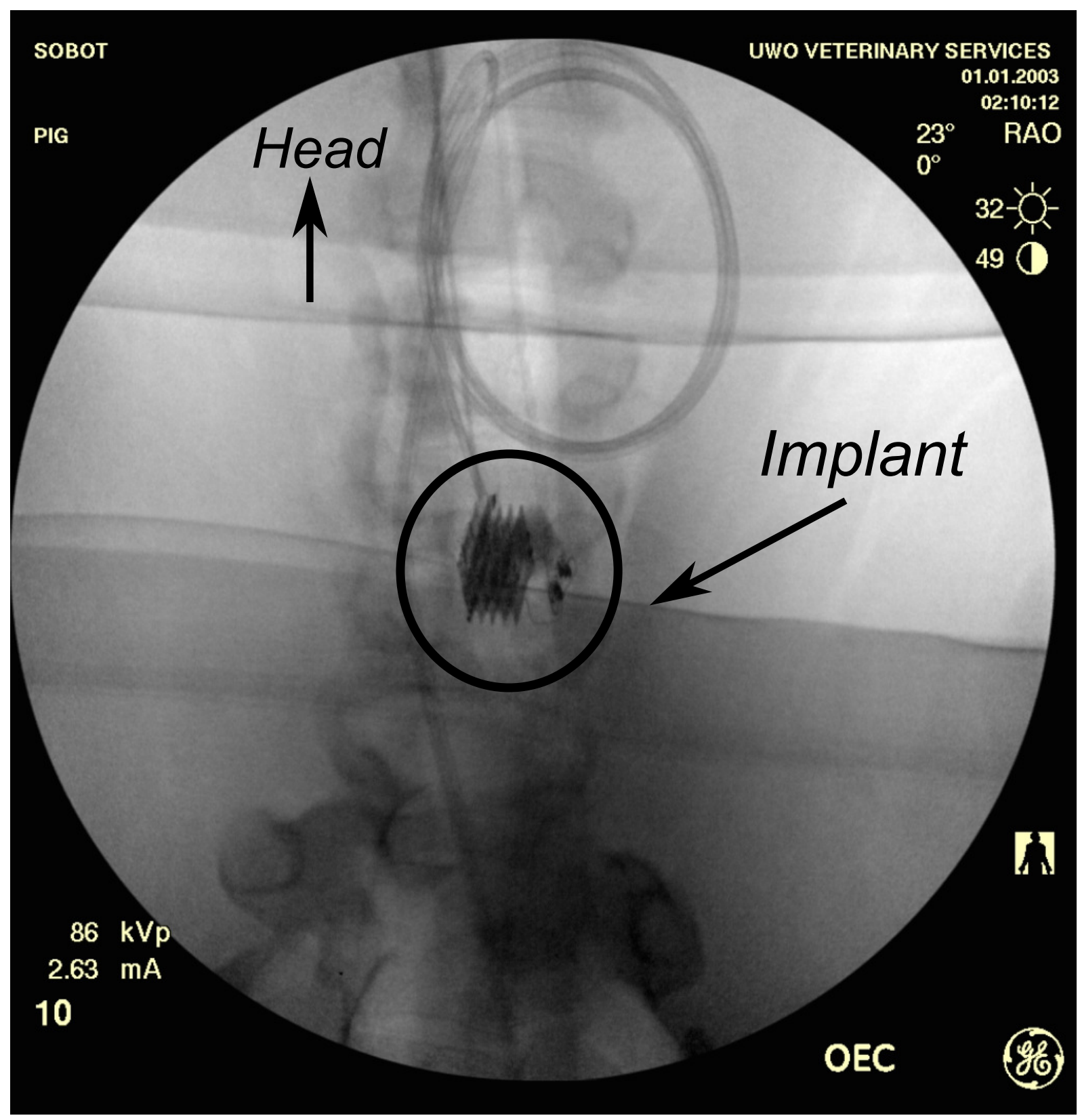

Figure 6.9: Fluoroscope image of wireless pressure-volume system implanted inside swine model. 
Table 6.4: Physiological and Physical Paramaters used in Volume Conversion Using Wei's Equation

\begin{tabular}{l|l|l|l}
\hline$\rho[\Omega-\mathrm{m}]$ & $\sigma / \epsilon$ & Segmentation Length $[\mathrm{mm}]$ & Stroke Volume $(\mathrm{SV})[\mathrm{ml}]$ \\
\hline 1.3 & $800 \mathrm{k}$ & 30 & 54 \\
\hline
\end{tabular}

by some surgeons. If the apex of the heart is chosen as a access route to the $\mathrm{LV}$, an alternative implant location should be explored. Ultimately, it is the decision of the surgeon of where the implant will be placed and how the left ventricle is accessed.

\subsubsection{Implant Data Collection}

Data collection is separated into two observation periods: a) acute and b) freely moving where a) represents data collection while the animal was under anaesthesia and b) represents live data collection from the freely moving swine model. During both observational periods, no abnormal clinical nor behavioural changes were detected. The subject was monitored with accordance to the approved ethics protocol.

In both observation periods, raw data was collected and converted to volumes offline. Pressure was converted in real-time as described in Section 5.3.1. Raw values were calibrated and converted to impedance using the method described in Section 5.3.2. Following calibration, the impedance was converted into admittance and finally into a volume using Wei's equation [228, 229,231], Section 3.1.2. In this study, the parameters: electrical conductivity and permittivity of the myocardium and blood resistivity are determined based on previous research [33, 231]. These along with the physical catheter measurements are used to the conversion from admittance to volume using Wei's equation (3.6), Table 6.4.

\section{Acute Observation}

The acute observation period represents the typical data collection environment utilized by commercial PV systems where the animal is anesthetized and a trained surgeon operates and controls the catheter placement within the LV. The implant was placed in duty cycle mode (2 
Table 6.5: Extracted Physiological Data From PV Loops

\begin{tabular}{l|l|l|l|l|l|l|l}
\hline $\begin{array}{l}\text { Observation } \\
\text { Period }\end{array}$ & $\begin{array}{l}\text { SV } \\
{[\mathrm{mL}]}\end{array}$ & $\begin{array}{l}\text { EDP } \\
{[\mathrm{mmHg}]}\end{array}$ & $\begin{array}{l}\text { ESP } \\
{[\mathrm{mmHg}]}\end{array}$ & $\begin{array}{l}\text { EDV } \\
{[\mathrm{mL}]}\end{array}$ & $\begin{array}{l}\text { ESV } \\
{[\mathrm{mL}]}\end{array}$ & $\begin{array}{l}\text { EF } \\
{[\%]}\end{array}$ & $\begin{array}{l}\text { HR } \\
{[\mathrm{bpm}]}\end{array}$ \\
\hline Acute & 50.9 & 10.42 & 118.30 & 77.32 & 26.44 & 65.8 & 156 \\
\hline Free Moving & 46.0 & 4.90 & 111.08 & 71.30 & 25.70 & 64.1 & 174 \\
\hline Distorted & 9.71 & 4.42 & 128.85 & 56.72 & 47.01 & 17.1 & 209 \\
\hline
\end{tabular}

min on and 2 min off) while the surgeon guided the catheter into the $\mathrm{LV}$, data was displayed in the custom LabView application on an external laptop. Data was observed prior to surgical closure to ensure proper data quality and catheter location within the LV, Fig. 6.10.

As noted above, the telemetry system was secured in the neck region, this was due to surgical recommendations. Although the telemetry system was more secure in this location, the additional tissue in this region impacted the default transmission power. This was not a major issue during initial measurement periods while the surgical opening was exposed allowing the antenna to radiate freely. However, once closed, the additional tissue greatly degraded the signal strength, and in turn reduced the receive range from meters to a few centimeters. Therefore, the output transmission level had to be increased to from $-12 \mathrm{dBm}$ to $1 \mathrm{dBm}$ to ensure a high quality data link.

Furthermore, various physiological events were monitored including the animals breath held. Average stroke volume (SV), End Diastolic Pressure (EDP), End Systolic Pressure (ESP), ejection fraction $(\mathrm{EF})$, and heart rate $(\mathrm{HR})$ during the acute observation period are shown in Table 6.5.

The goal of this study is to evaluate the PV system in a freely moving animal and not to perform drug based studies. Thus, there were no drug infusions in this study, only baseline measurements are made while the animal is anaesthetised. Furthermore, multiple animal studies are required for a more comprehensive physiological study. In this study, aspects of the PV telemetry system are evaluated with respect to its overall performance and not the specific physiological changes in the animal. However, it is still greatly important to evaluate the efficacy of the PV telemetry system versus traditional tethered approaches. It is only valid to 


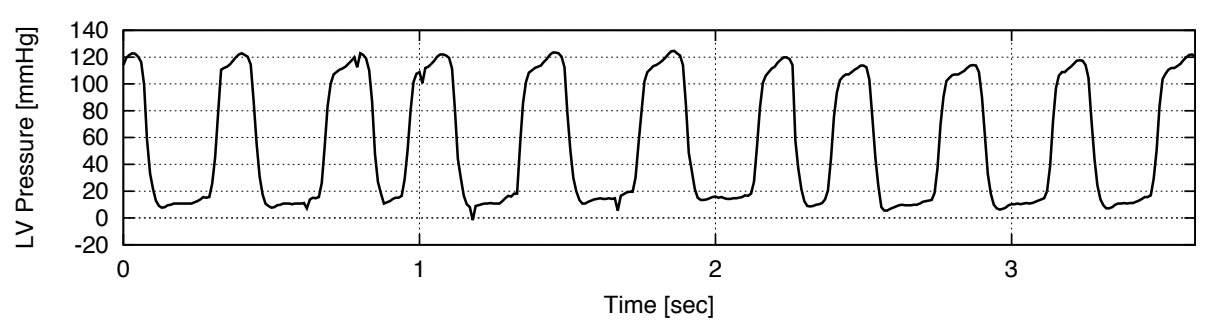

(a)

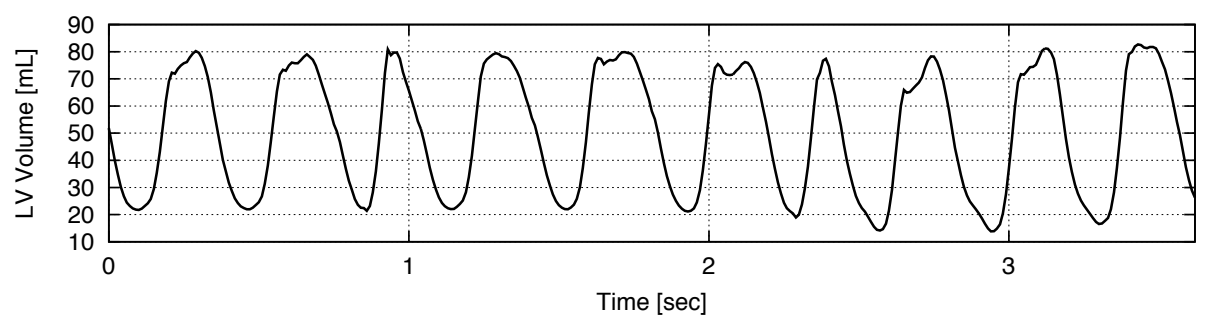

(b)

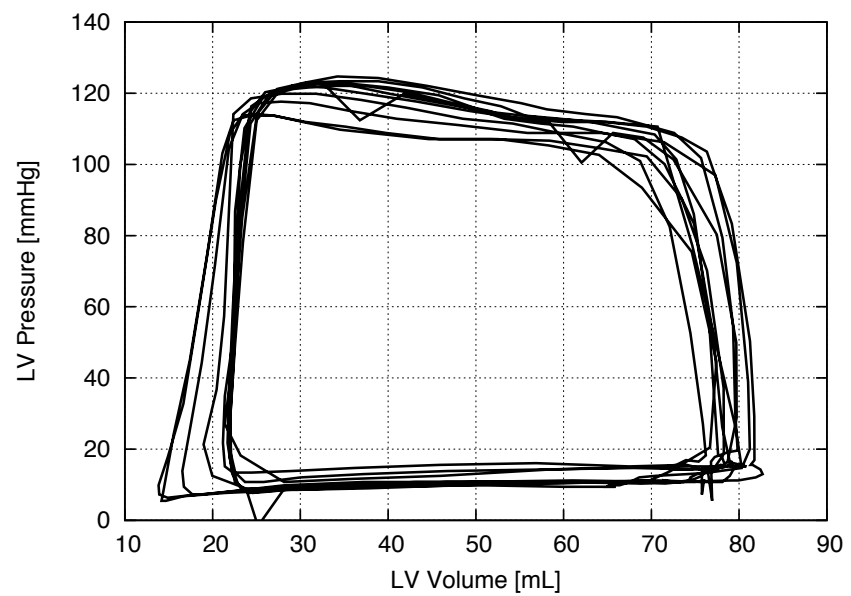

(c)

Figure 6.10: A sample of data collection during acute measurement phase where a) LV pressure; b) LV Volume; and c) PV loop. 
Table 6.6: Comparison of Physiological Paramaters with those from [33]

\begin{tabular}{l|l|l|l}
\hline Works & $\begin{array}{l}\text { EDV } \\
{[\mathrm{mm}]}\end{array}$ & $\begin{array}{l}\text { ESV } \\
{[\mathrm{mm}]}\end{array}$ & $\begin{array}{l}\text { EF } \\
{[\%]}\end{array}$ \\
\hline$[33]$ & 72.7 & 39.8 & 47.13 \\
\hline $\begin{array}{l}\text { This work } \\
\text { (Acute) }\end{array}$ & 77.32 & 26.43 & 65.8 \\
\hline
\end{tabular}

compare approaches in the typical measurement environment of an anaesthetised subject with a fully open wound. Ideally, the most suitable method of comparison would be to insert a traditional catheter into the LV that is tethered to an external Advantage ADV500 processing unit (Transonic Scisense Inc.), Fig. 1.4][3], and perform baseline measurements, Chapter 1. This setup is used in most measurement environments. However, by doing this, it would introduce additional risk to the animal and would jeopardize the main goal of the in-vivo study. Instead, the implantable PV system's measurement values, more specifically, volumes are compared to ones found in [33]. Kutty et. al. [33] presented a detailed study comparing the LV volumes of 8 pigs measured using the admittance technique (same catheter technology used in this thesis) and using real-time three-dimensional echocardiography. This paper is chosen as a comparison because similar catheters and equipment are used as what is used in this in-vivo study. Furthermore, similar sized pigs are used in [33] as is in this study. Kutty et al. demonstrates that admittance measurements have acceptable correlation with real-time three-dimensional echocardiography. Thus, it is valid to compare our measured data with the baseline data for admittance found in [33] for initial validation only. A comparison of End-diastolic volume (EDV), End-systolic volume (ESV) and EF values from [33] and measured data from the acute observation period is shown in Table 6.6. Note, the values from [33] used in Table 6.6 are averaged values taken from 8 pig measurements during baseline, Table 1 in [33]. The volume measurements from the PV telemetry system correspond with those measured using the traditional admittance catheter approach.

The variation in the ESV and EF is most likely due to animal to animal variability and does correspond with individual pig measurements found in [33]. It is expected that our PV 
telemetry system would exhibit similar results over a larger animal study. The comparison is not valid for infusion as our study does not involve additional drugs. It should be noted that, as every animal is slightly different, each will exhibit slightly different physiological parameters. Furthermore, depending on the specific surgery performed, it may impact measurement results. A larger study involving multiple animal subjects is planned in future work to further evaluate the PV system and to do a full comparison with other measurement methods. However, it can be shown that our implant achieves the same data quality as traditional methods while unders similar measurement and surgical conditions.

A similar study published by Van Hout et al. [236], compared admittance based pressurevolume loops vs magnetic resonance imaging in 14 pigs. The results of this study indicate that admittance based measurements moderately overestimates LV volumes but is very accurate for ejection fraction when compared to MRI. A different measurement protocol was used in [236], thus it cannot be compared directly with this work. All animals underwent a procedure to simulate a myocardial infraction followed by a 8 week recovery time. No baseline measurements are provided. However, this represents a valid resource when comparing admittance based PV loops vs MRI.

\section{Free Moving Observation}

The animal was allowed to recovered fully before data collection to occur. This was to ensure that any effect of the anaesthesia left in the animal's system did not impact measurement results. Furthermore, it was critical during this time to monitor the animal closely by veterinarian technicians to ensure no health complications occurred. This period lasted approximately 2 hours and no significant health issues were observed during recovery. The pig was paced in a $(206 \mathrm{~cm} \times 107 \mathrm{~cm} \times 187 \mathrm{~cm})(\mathrm{LWH})$ steel cage for further health and data monitoring.

Data was continuously observed for 24 hours in the postoperative period via the RF link to the external unit, Fig 6.11. The implant was placed in various duty cycles to ensure battery life was extended to maximum. Initial data quality and PV loops while the animal was freely 


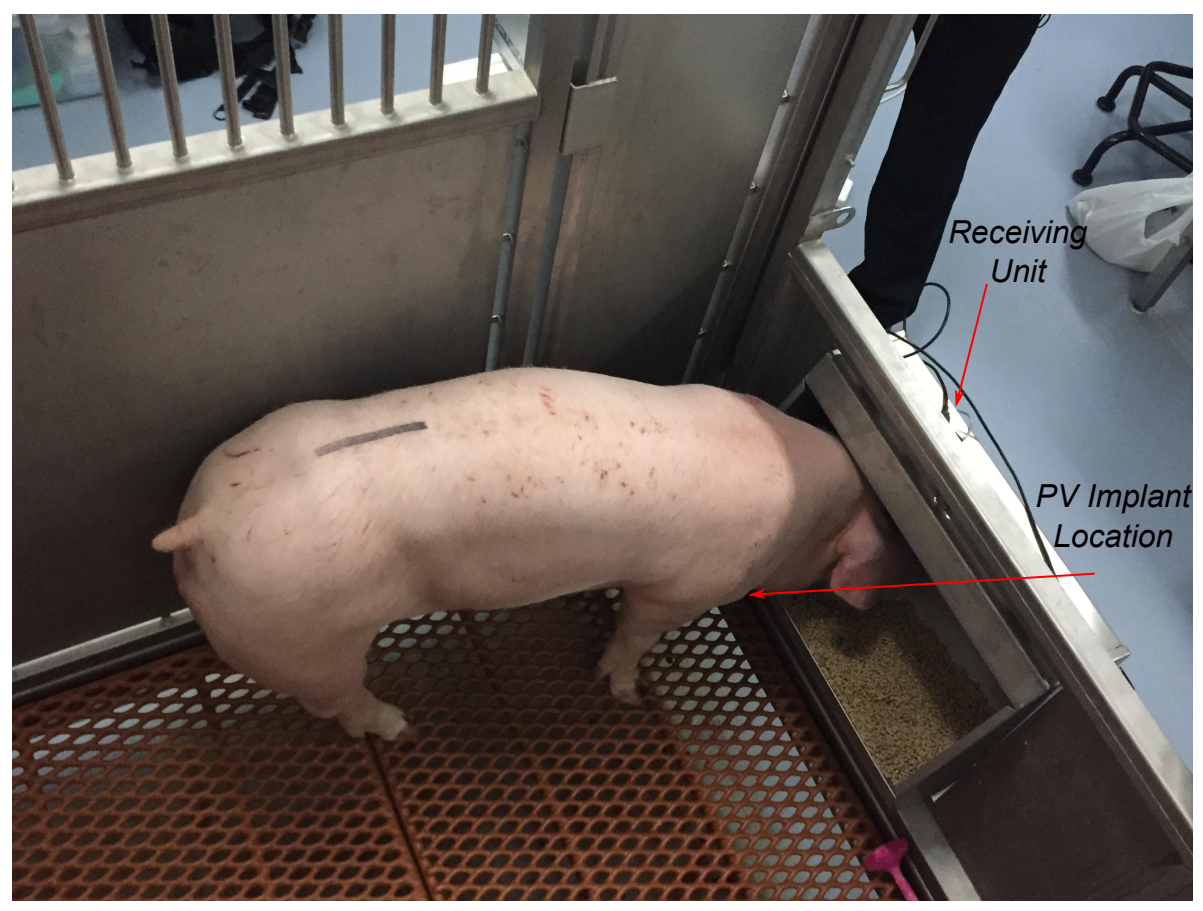

Figure 6.11: Live data collection of left-ventricle pressure-volume signals from a freely moving swine model.

moving around inside its cage corresponded to that of the acute study. For example, measured LV Pressure and Volume signals are shown along with the corresponding PV loops while the subject was walking and eating for a 12 second period, Fig. 6.12. Similar measurements were recorded throughout this period. A slight reduction in measured stroke volume, EDV, ESV and EF was detected while monitoring the freely moving animal, Table 6.5. The variation in physiological signals is most likely attributed to the animal still feeling the effects of of the surgical procedure, including general pain and the effects of a catheter inserted in its cardiovascular system. All medication delivered during this period is in accordance with the approved ethics protocol.

Distortions in the volume signal were observed while the subject would perform neck movements and when abruptly sitting down. This movement was consistently observed throughout this period and believed to have occurred due to the volume catheter rings contacting the LV walls during movement. The consistent movement causes the catheter to move within the LV and either become fixed on the LV wall or not centered within the LV. This directly effects 


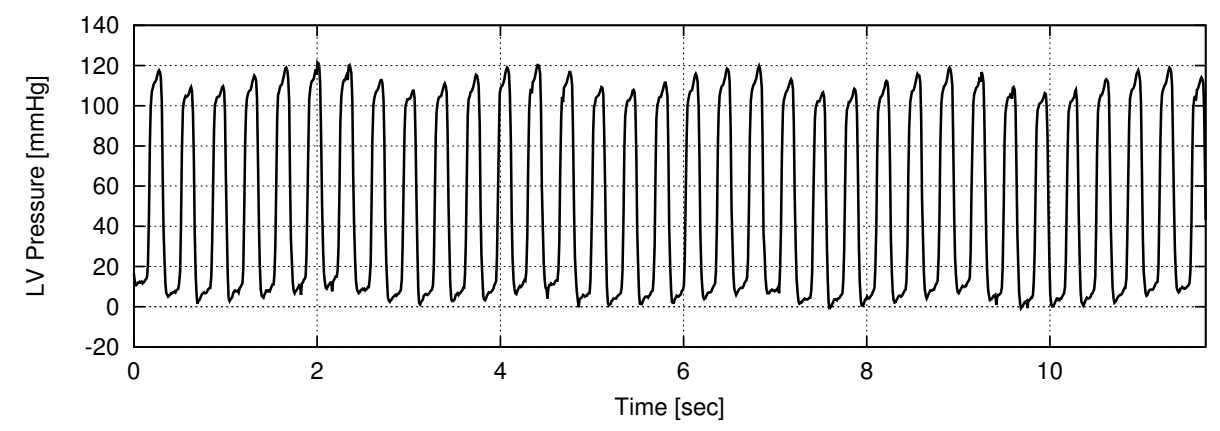

(a)

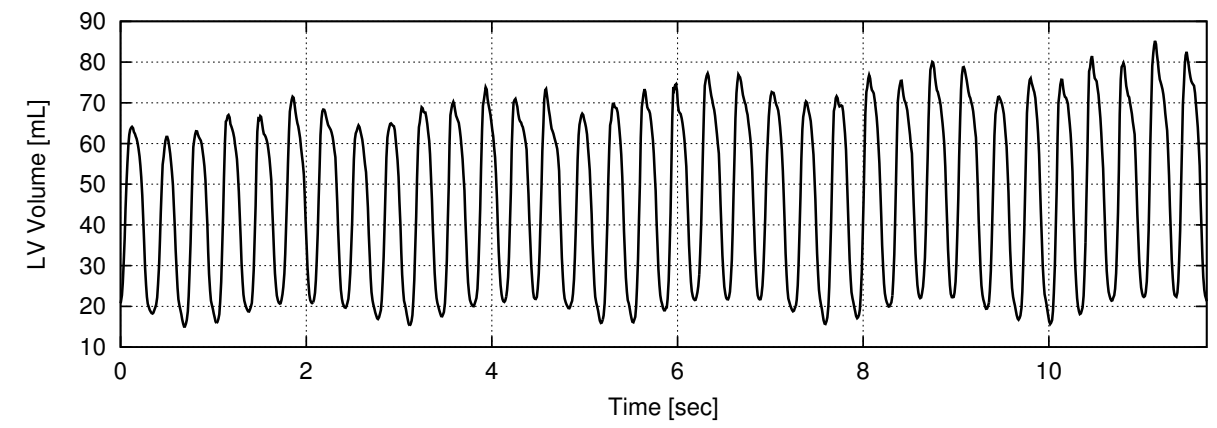

(b)

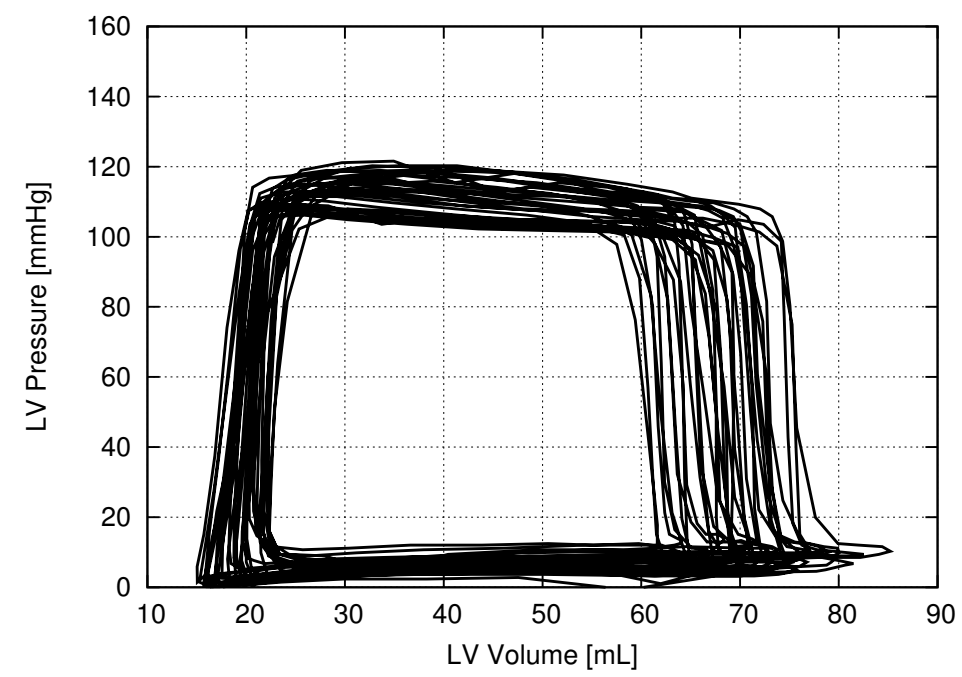

(c)

Figure 6.12: A sample of 12 seconds of data collection during free movement where a) LV pressure; b) LV Volume; and c) PV loop. 


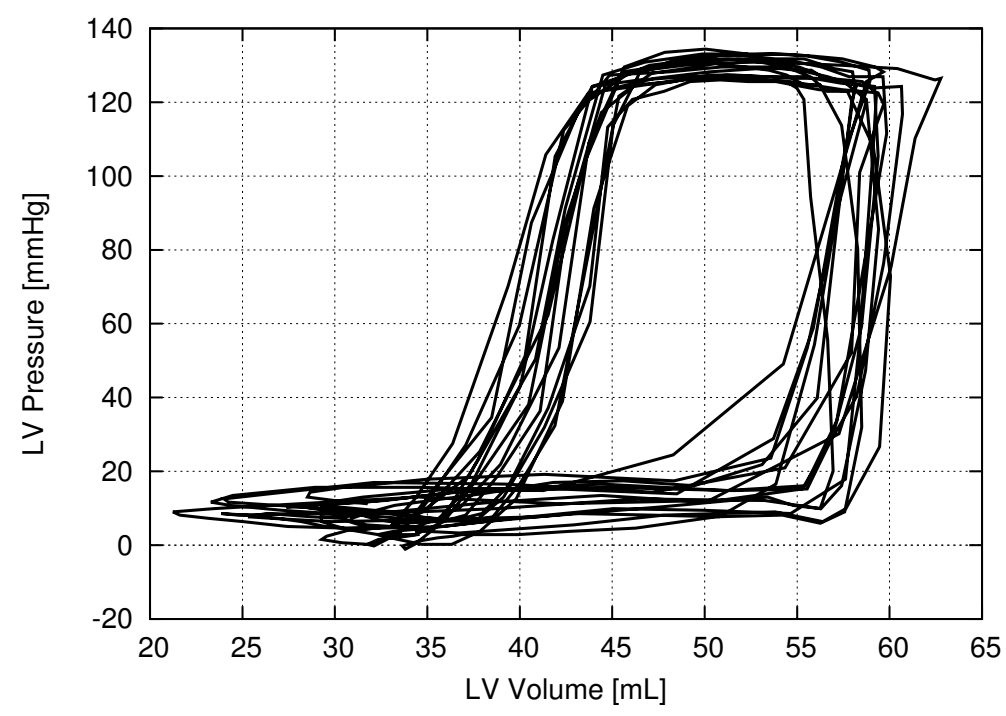

(a)

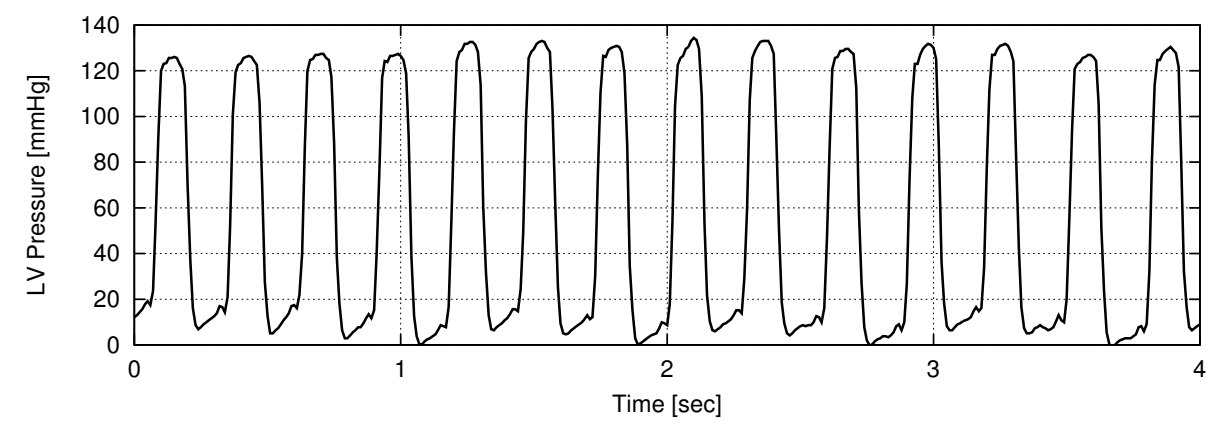

(b)

Figure 6.13: A four second sample demonstrating a) distorted PV loops; b) LV pressure.

the measured volume signal by significantly distorting the signal until not being measured at all. However, the pressure signal is unaffected by the catheter movement as it is a MEMS based pressure sensor and not reliant on an electric field. Furthermore, the increased movement of the catheter within the LV created an increase in HR from the additional stress on the animal. An example of the distorted PV loops are shown in Fig.6.13 along with the measured LV pressure. The extracted physiological parameters for the distorted PV loops demonstrate that once the catheter becomes dislodged from the center of the LV, the extracted parameters that rely on volume are no longer accurate, heart rate can be determined by pressure only, Table 6.5 .

The study lasted for a total $48 \mathrm{~h}$ which included $24 \mathrm{~h}$ of data collection and a combination of $24 \mathrm{~h}$ of additional recovery and monitoring time before and after data collection. The 
approved ethics protocol dictated the total number of days of the study, along with approved measurement equipment and drugs that were administered. Thus, additional live Fluoroscopy, Computed Tomography (CT), or other scans following the surgery and during the monitoring period were not allowed. This resulted in the lack of a full internal view of implant and catheter system after a 2 day monitoring period.

Due to the length of the study it was determined to optimize battery life and measurement data. For the duration of the monitoring period the implant remained in duty cycle mode measuring data every 2 min and off for 18 min with a receive cycle occurring every $1 \mathrm{~h}$. This lead to a total battery life of approximately $48 \mathrm{~h}$ including initial implant configuration, encapsulation, receive cycles, higher Tx power $(+1 \mathrm{dBm})$, and measurement time of the study.

\subsubsection{Implant and Catheter Tissue Impact}

Another goal of this study was to determine the effect that the implant had on the surrounding tissue as well as the effects of the catheter in the left ventricle and surrounding areas. A macroscopic observation of the tissue was completed following euthanasia, and a full histopathology study was completed on select areas of tissue.

\section{Macroscopic observation}

After the euthanasia (based on ethics protocol), a partial necropsy focusing on the heart and immediate large vessels was performed. Initial observations of the implant location indicated clotting around the implant and coiled catheter, Fig. 6.14. The implant, heart, and portions of the carotid artery were separated from the rest of the body for further analysis, Fig. 6.15. The heart, mainly the left ventricle, was cut open to inspect damage caused by catheter movement throughout the study, Fig. 6.16. Areas of bruising and superficial hemorrhage; clot adhesion in the heart; and large vessels were detected, Fig. 6.16. Furthermore, by visually inspecting the catheter, it was confirmed that the catheter tip (pig tail) did break off during surgery, Fig. 6.16.

Additional areas of clotting were determined in the carotid artery next to the area where the 


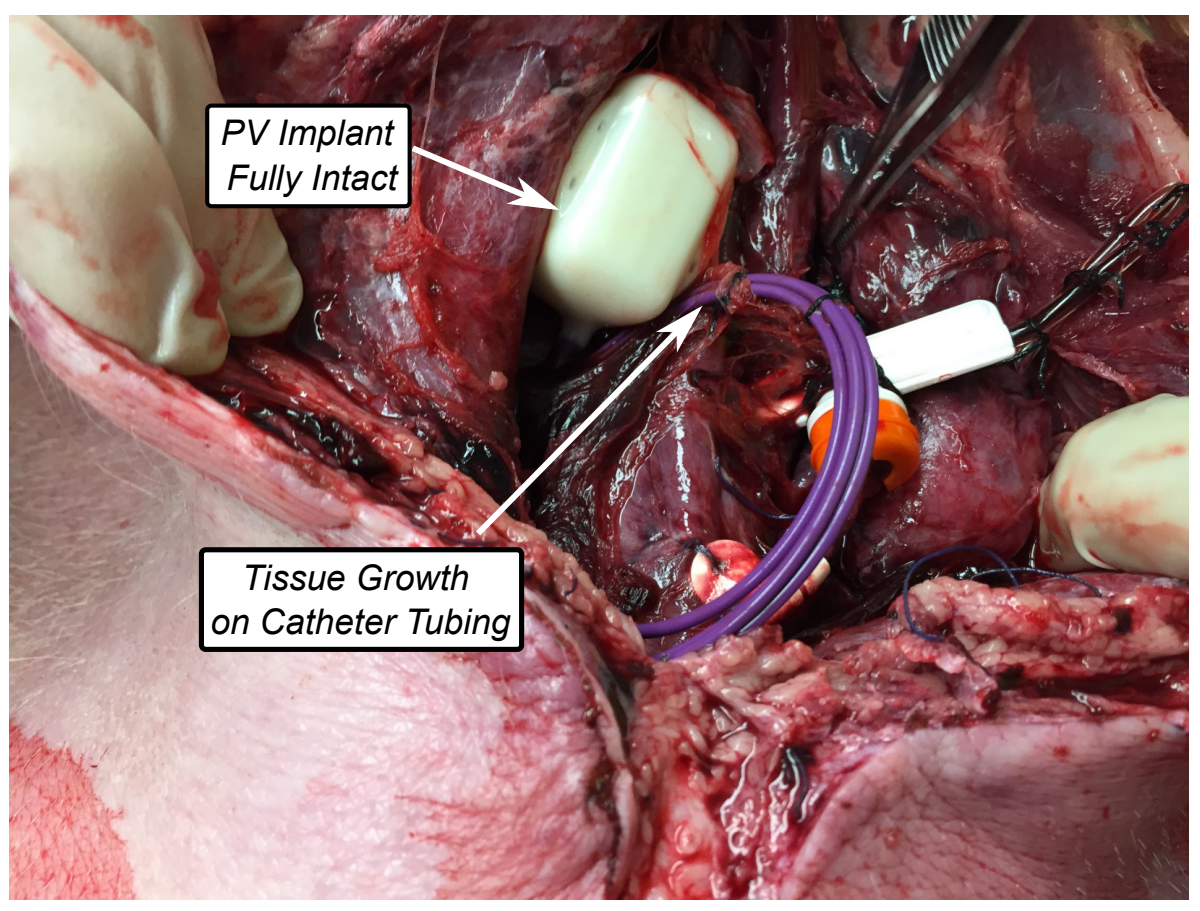

Figure 6.14: Initial implant explant. Tissue growth observed on catheter coil.

catheter was inserted, Fig. 6.17. Tissues were collected from areas that macroscopic lesions were identified. Tissue samples of the left cardiac ventricle, aortic valve, and carotid artery were processed for microscopic evaluation.

\section{Tissue sample preparation for microscopic evaluation:}

Tissue preparation was completed by Veterinary Services technicians at Western University. All tissues were placed in tissue cassettes in 10\% Neutral Buffered Formalin (Fisher Scientific Company, Kalamazoo, USA). After 5 days, the tissues were taken for tissue processing using an automated tissue processor (Leica ASP 300 Tissue Processor). The cassettes were loaded into the tissue processor (Leica ASP 300). Dehydration and paraffin infiltration were performed. Within 1 hour of completion of this procedure, the tissues were embedded in paraffin using the embedding console system (THERMO Shandon HISTOCENTRE 3) and assorted base molds (Fisher HistoPrep Stainless-Steel). Once the paraffin solidified, the embedded tissue blocks were removed from the base molds and any excess wax was scraped from the 


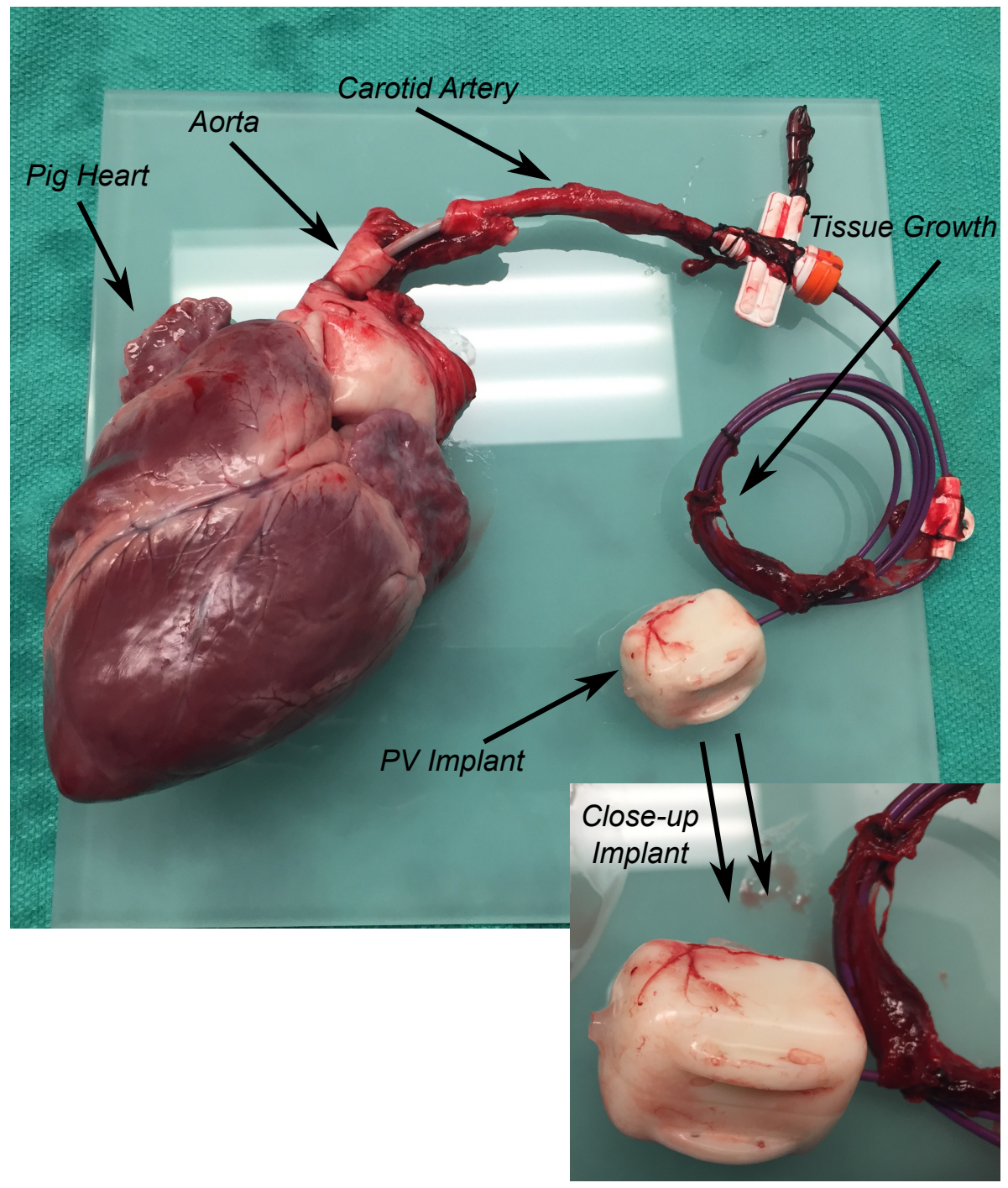

Figure 6.15: Explanted implant, heart and portions of the carotid artery. Close-up of implant and tissue growth is shown. 


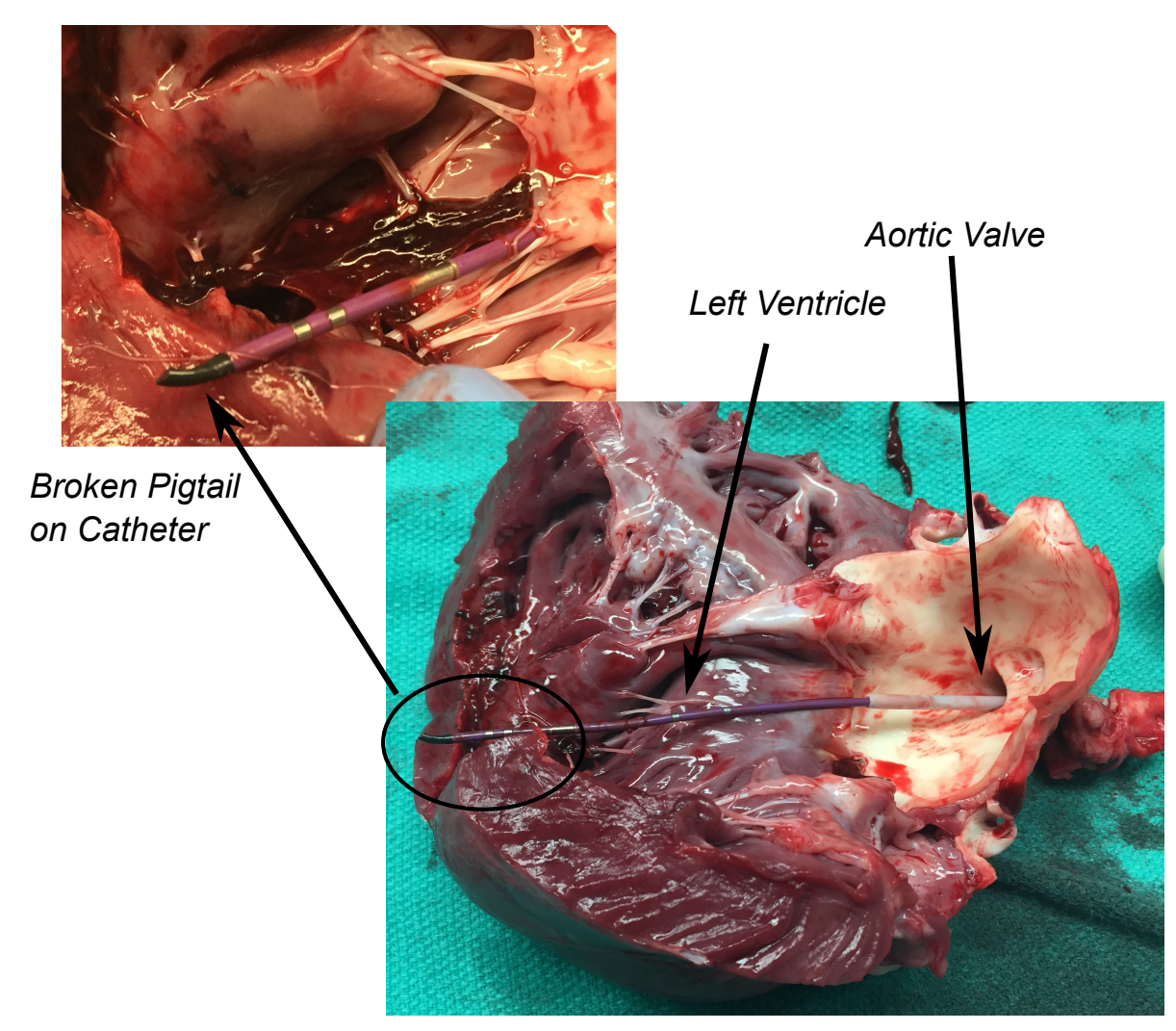

Figure 6.16: Dissection of left ventricle displaying broken catheter tip and aortic valve.

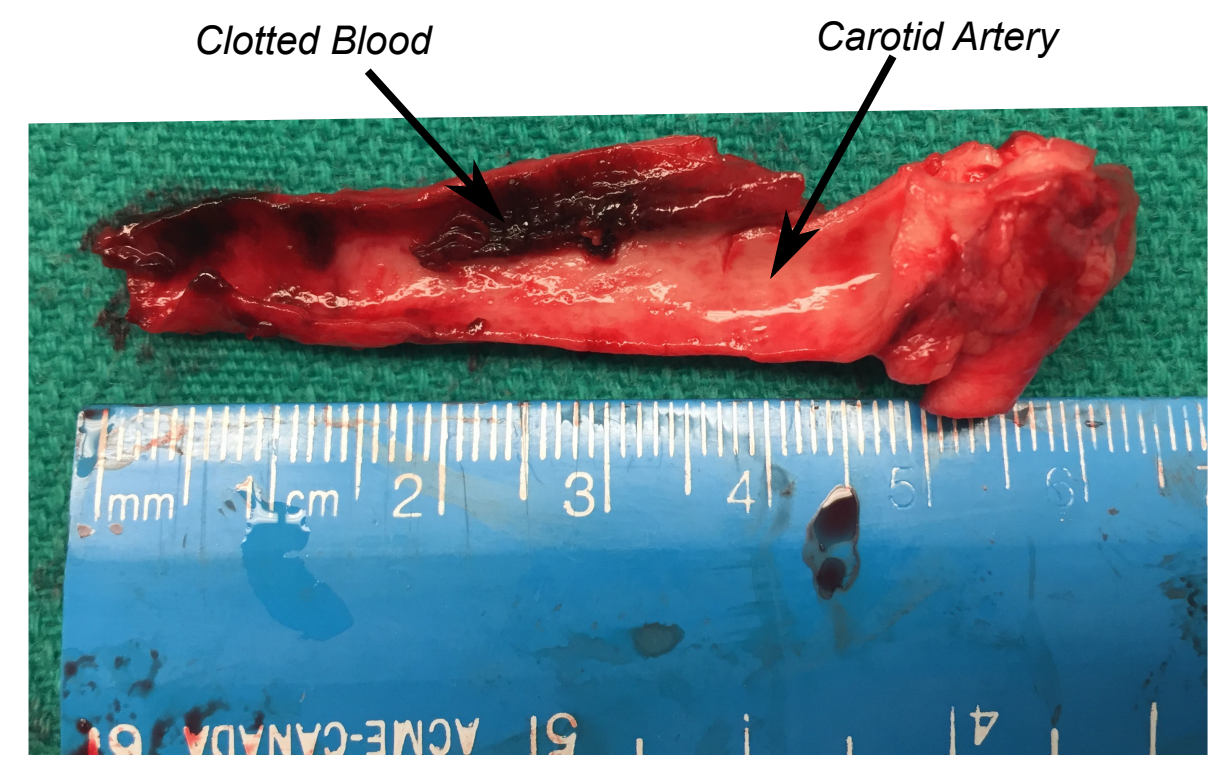

Figure 6.17: Carotid artery with clotted blood. Tissue sample used in microscopic evaluation. 


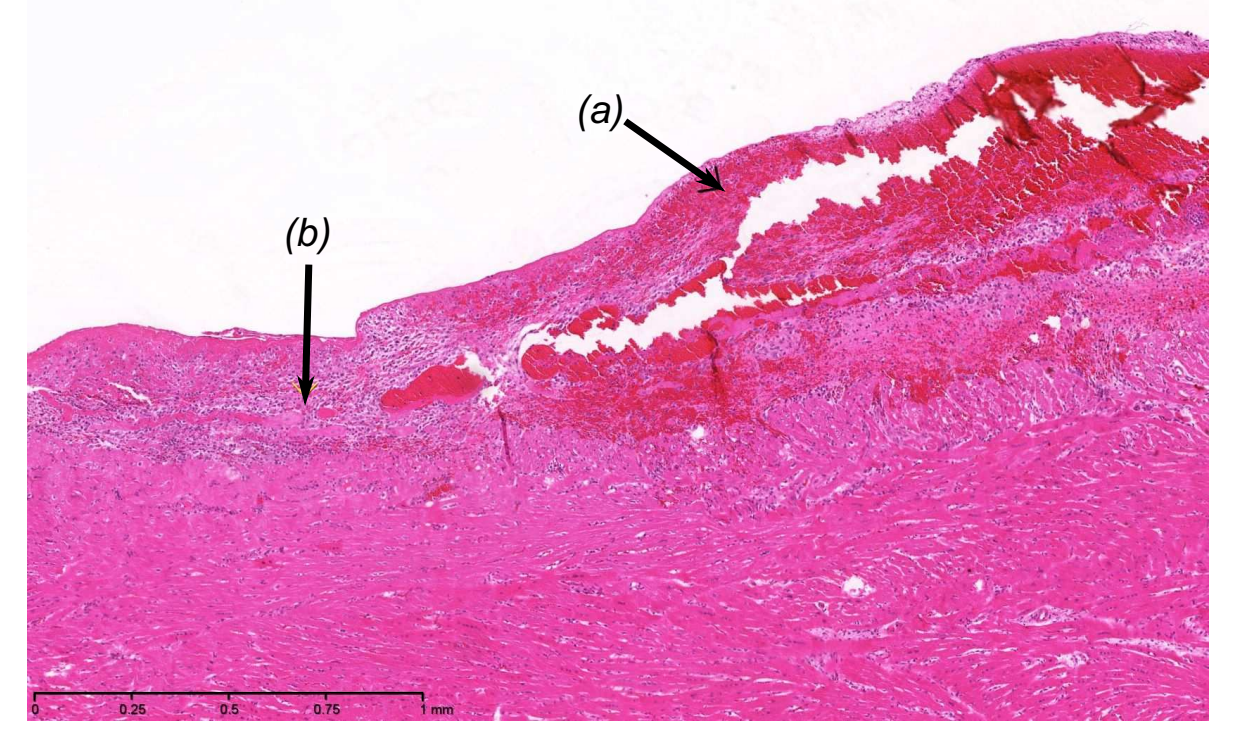

Figure 6.18: An example of Left ventricle: a) ulceration and hemorrhage with b) early granulation tissue as noted by a pathologist.

edges. Tissue blocks were cooled on ice. Sectioning of the blocks was performed on a rotary microtome (Leica RM2255). Following trimming, $5 \mu \mathrm{m}$ sections of each of the tissue blocks were taken and mounted on positively-charged glass slides (Fisher Scientific). Slides were heat-fixed in an incubator overnight and stained the following morning in an automatic stainer (Leica Autostainer XL) using hematoxylin and eosin Y solutions. This work was completed in partenership with The Toronto Centre for Phenogenomics (TCP) - CMHD Pathology Core and Dr. Hilbert Adissu (Pathologist) and Dr. Alex El-Warrak (Surgical Team). All work was completed by trained technicians.

\section{Histopathology Results}

A total of thirteen slides were provided by TCP with a full detailed report and analysis for the LV (6 slides), aortic valve (4 slides) and carotid artery (3 slides). The results of the microscopic evaluation corroborated with the macroscopic observations. It was found that segmental ulceration and hemorrhage occurred in the left ventricle endocardium, aortic valve, and the carotid artery, Fig. 6.18, Fig. 6.19 Fig. 6.20.

The results of the histopathology study indicate that the lesions are consistent with a super- 


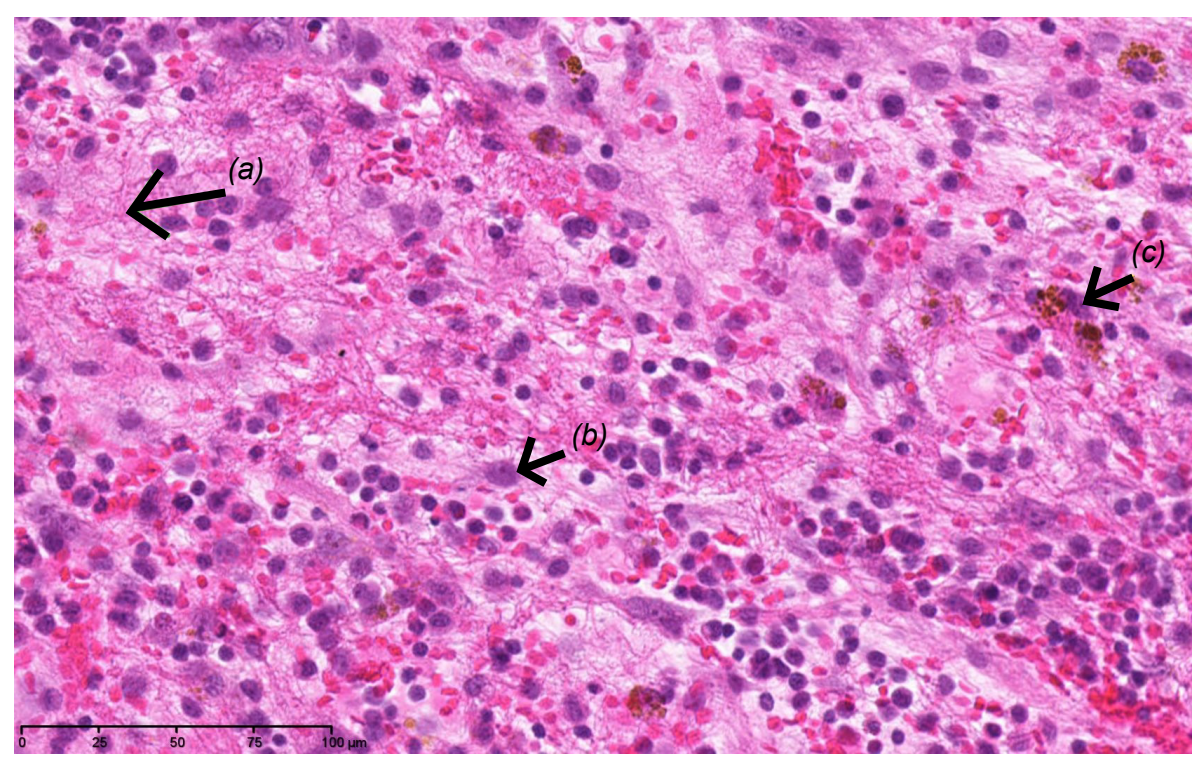

Figure 6.19: An example of Aortic Valve with ulveration and hemorrhage with early granulation tissue. Issues of concern as noted by a pathologist: (a) fibrin, (b) activated fibroblast, and c) hemosiderin.

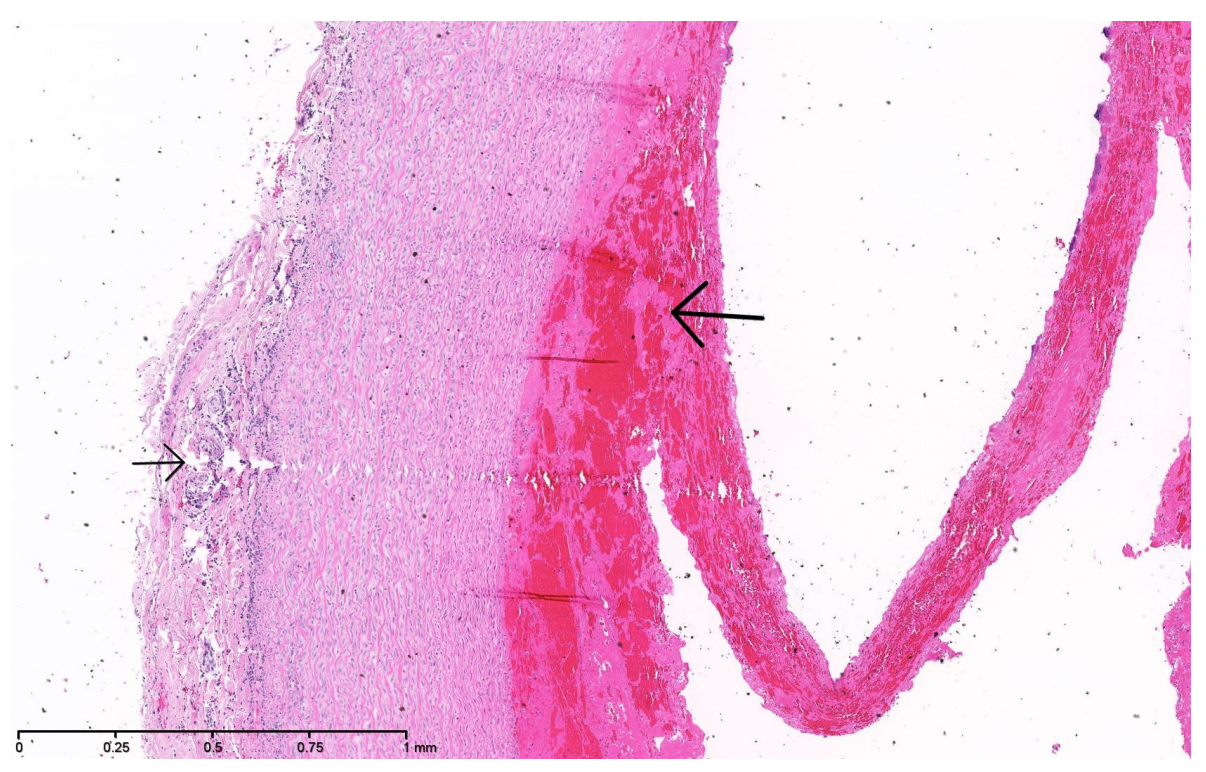

Figure 6.20: An example of Carotid Artery with ulceration and hemorrhage, necrosis of intima, disruption and inflammation of the tunica adventitia as noted by a pathologist. 
ficial traumatic injury/laceration of the endothelium and endocardium by a foreign body/device (catheter / implant). The superficial myocardium is minimally involved from inflammatory and reparative processes (granulation). These results also indicate that the lesion was recent, i.e., from this experiment and not a pre-existing condition due to the presence of early granulation tissue and hemosiderin, Fig. 6.19.

\subsubsection{In-Vivo Study Discussion}

The histopathological findings corroborates with the assumption that the lesions (tissue damage) may have been caused by the catheter insertion and also by the tip of the electrode that broke while insertion. Further analysis of the damaged catheter, Fig. 6.16 was completed postmortem. The initial damage to the catheter tip occurred when the smaller $5 \mathrm{~F}$ pigtail became entrapped inside the introducer. Furthermore, the forces of the LV caused further damage to incur on the catheter tip. The catheter could have been replaced to minimize the foreign body inflammatory and reparative processes (granulation). However the decision to not do so was made based on the limited availability of telemetry system implants and the positioning achieved while insertion and the optimal initial readings obtained. To prevent catheter failure, better mating of the introducer and the outer diameter of the pigtail is recommended.

Although the catheter utilized in this study performed correctly throughout the acute and free-moving observational periods, over time the movement of the subject created significant impact on the measurement quality of the volume signals, Fig. 6.13. Furthermore, the catheter itself is designed for acute studies and not designed for free-movement of the subject. Therefore, a catheter that is designed and optimized to be used with an implanted PV telemetry system is required for long-term chronic use within the LV. Future studies should be undertaken to determine full effect of catheter-heart interaction. These studies should include multiple animal subjects (large and small) as well as a longer term study i.e., 1 month.

In general, the PV telemetry system performed quite well. There were however, limitations and issues throughout the measurement period. As previously mentioned, the transmission 
power had to be increased due to the additional tissue. Furthermore, the location of the antenna within the enclosure created its own set of challenges. As the antenna radiated outwards, it was essential that the implant remain facing out toward the exterior of the body. It was unknown if the implant rotated within the cavity further degrading the transmission power. It is important to create a marker to indicate antenna direction on the enclosure. Another issue which impacted the battery life was the start up sequence. The implant requires the battery to be connected at time of enclosure, thus consuming power possibly days in advance of a surgery. This directly effects the total time of a study. To overcome this issue, a magnetic switch should be used to control the power delevery to the implant. In this version, of the implant, the battery size was kept at a minimum to conserve space. If a longer study is required a much larger battery should be used or large reductions in power consumption must be made. Another issue is the lack of on board calibration and full detailed PV loops within the custom measurement application. These issues among others should be addressed in future versions of the PV telemetry system to improve data quality and measurement time.

A comparison of the presented PV system to published works is summarized in Table 6.7 . The PV telemetry system designed and tested in this thesis achieved significant reduction in the overall size and weight along with reported power consumption. For instance, in comparison with the latest reported research [25], this design is $58 \%$ smaller in volume, $44 \%$ in weight and $55 \%$ reduction in sampling power. In addition, future versions of the device will utilize sub-GHz bands to decrease tissue absorption and increase transmission range. Subsequently, with the addition of a custom designed PV catheter suited for implantable applications the PV system will be further improved. The successful data obtained with this initial experiment and the uniqueness of being the one of the first real-time recordings of a conscious swine subject in its natural environment confirms the need for longer term observational periods. 


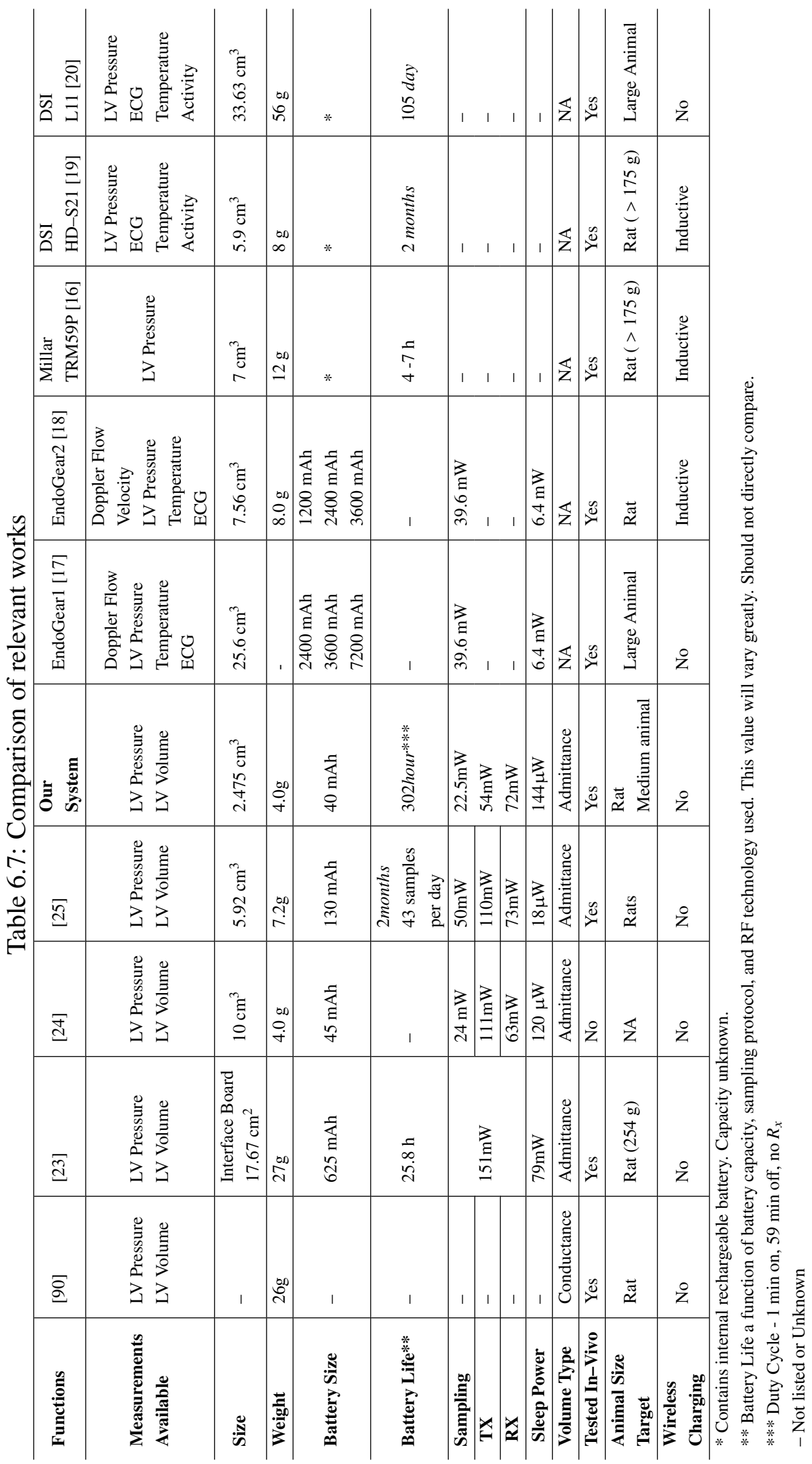




\subsection{Conclusion}

In this chapter the custom measurement application built in Labview was presented. In-vivo results are presented that demonstrate the validity of our RF pressure-volume telemetry system. The PV sensor was implanted into a pig's left ventricle while the encapsulated unit was secured in the neck region. Live data was captured in both an acute and a freely moving setting over a 24 hour period. Further improvements in catheter design as well as improvements in the implantation methodology will improve the data quality and safety of the subject. This real-time implantable technology allows for researchers to quantify cardiac pathologies by extracting real-time pressure-volume loops, wirelessly from within freely moving subjects. 


\section{Chapter 7}

\section{Conclusion}

The aim of this thesis is to develop a miniature, low-power wireless implantable telemetry system that is designed to measure left ventricular pressure and volume physiological data. In correspondence to this goal, a fully implantable telemetry system capable of measuring leftventricular blood pressure and volume (PV) was created. This chapter provides a summary of the main contributions made by this thesis, plus suggestions on future work.

\subsection{The Contribution of the Thesis}

In this thesis, the following achievements are made:

- In this thesis a comparison study of the three conductance-to-volume equations is presented, that revealed Baan's linear equation (3.1) is too simplistic and significantly overestimates volume and is not recommend. Dubois' equation (3.8) is based on the analytical equation (3.4) and is therefore very inaccurate. It consistently underestimates volumes and its use is also not recommended. Wei's equation (3.6) is the most accurate out of the three and is therefore the only currently viable equation for conductanceto-volume calculations. Overall, it is experimentally shown that there is an average of $26.25 \%$ error in volume measurements when using Wei's equation (3.6) using a sample 
set of commercial $1.2 \mathrm{~F}$ catheters, which is much greater than the industry accepted value of $15 \%$. Measured data is used to develop a calibration equation 3.11 that can be used to decrease measurement error to $5 \%$ when using Wei's equation (3.6).

- To overcome the challenges and limitations that existing commercial PV systems have, a fully implantable telemetry system was developed. The PV telemetry system architecture consists of four system level blocks (modules) that share a common data and DC power bus: power regulating electronics (PWR), signal processing and control unit $(\mu \mathrm{C})$, RF transceiver ( $\mathrm{Rx}-\mathrm{Tx})$, and PV sensor interface [239]. A PV interface was designed to provide stimuli and reference signals to the pressure and volume sensors. The pressure sensor is based on a MEMS Wheatstone bridge structure that is excited by a DC voltage reference. The voltage reference and measurement are completed through a commercial IC for pressure sensing applications. Similarly, the volumetric section of interface uses the admittance methodology to measure the blood volume in the LV, that is, both magnitude and phase of the measured voltage are extracted. The volumetric sensor itself is excited by an $\mathrm{AC}$ current reference. Phase sensitive detectors along with a quadrature oscillator are used to extract DC In Phase and Quadrature voltages that are proportional to the raw in phase and quadrature voltage signals at the PV sensor. This approach allows for the direct extraction of the phase signal where as traditional approaches require additional hardware and pulse width analysis to determine the phase. Data capture and processing is completed by a Texas Instruments (TI) MSP430 with the analog data from the PV sensor interface digitized by the internal 10-bit A/D converter. RF transmission from the implant to the external unit is completed by a low-power TI CC2500 radio transceiver. The RF data is transmitted at $2.45 \mathrm{GHz}$, thus, allowing the system to work in the Industrial, Scientific and Medical (ISM) band. Furthermore, the control architecture is presented indicating an average current consumption of $40 \mu \mathrm{A}$ (over 1 sample) and $5.59 \mathrm{~mA}$ in continuous sampling mode. In addition, various measurement cycles were explored. The physical implementation of the PV implant was designed with the pri- 
mary aim to explore the physical limitations of the state-of-the-art discrete components technology in respect to the minimum possible physical size, and the system's power consumption requirements. The size of the complete wireless PV telemetry system, including the Li-Poly battery, is $15 \mathrm{~mm} \times 15 \mathrm{~mm} \times 11 \mathrm{~mm}$ (i.e., $2.475 \mathrm{~cm}^{3}$ ) and it weighs $4.0 \mathrm{~g}[239]$.

- A phantom tissue study was performed to model the effects of implant conditions and to determine the appropriate RF transmit power from the PV prototype [257]. The conductivity and relative permittivity of the phantom tissue are modelled to match that of muscle with a conductivity $\left(\sigma_{m}\right)$ of $2.21 \mathrm{~S} / \mathrm{m}$ and relative permittivity $\left(\epsilon_{r}\right)$ of 47 at $2.45 \mathrm{GHz}$ [148]. The phantom tissue was cut into a $4 \mathrm{~cm} \times 4 \mathrm{~cm} \times 3 \mathrm{~cm}$ structure, where the antenna is inserted. This model emulates the tissue through which the transmitted RF signal must pass through while being transmitted from within the body. The outcome of this study helped to determine the minimum RF output power that the transmitter $\left(\mathrm{T}_{x}\right)$ needs to provide to still receive data from the implant while having minimum power consumption in a typical medical measurement environment, i.e., instrument noise, non ideal antenna matching, spectrum interference, etc.

- Implant capsules were designed to accommodate the manufactured implantable PV telemetry system, they consist of a front port hole for catheter access, a side antenna access hole, and are split into top and bottom halves, where the battery and the implant system are placed in the bottom half. A custom SolidWorks package model was 3D printed in four different plastic and one metal packages using the following materials: 316L stainless steel, ABS, RDG720 Transparent Acrylic, and ABS-M30i. Each material was evaluated for leaks by a 98 day water immersion study [258]. The stainless steel capsule provided the highest precision among the four materials however, additional post-processing time is required. For the in-vivo experiment, we used a package with suture holes so that the implant capsule is secured directly to the animal. This package was printed in ABS- 
M30i plastic to ensure bio-compatability with the subject's tissue.

- Complete calibration of the pressure and volume sensing front-end was presented. The MEMS absolute pressure sensor was calibrated inside a fluid-filled pressure chamber that allows for variations in temperature and pressure. Four point calibration was used to calibrate for the variation of the Wheatstone bridge offset over temperature and variation of the bridge gain over temperature thereby providing $1^{s t}$ order temperature compensation. The sensor was calibrated over a $450 \mathrm{mmHg}$ pressure range $(-135 \mathrm{mmHg}$ to 315 $\mathrm{mmHg})$ and over a $20.4^{\circ} \mathrm{C}$ temperature range $\left(22.8^{\circ} \mathrm{C}-43.2^{\circ} \mathrm{C}\right)$. Two calibration methods for the volume electronics were presented. The average errors across the calibration range for the impedance magnitude and phase for each method are: 1) $0.211 \Omega$ and $0.2^{\circ}$; and 2) $0.2139 \Omega$ and $0.2263^{\circ}$. It can be seen that the second method is slightly less accurate in producing calibrated values however, it is less computational intensive thus faster to implement. For the experiment described in this thesis, the first calibration method was used as all data processing was done offline thus computation complexity was not an issue and data accuracy was of great importance however, for on-chip calibration implementation the second method should be used.

- To receive the transmitted data signals from the implanted telemetry system an external receiving unit (Rx Unit) that consists of a microcontroller, wireless transceiver, and hardware UART board was designed. The data transmitted from the implant is received via the hardware wireless receive block and sent to UART module to be transmitted to a PC. In this thesis, a Windows based system was used and can display this data in a traditional terminal window or a custom based application like LabView. Both options were explored and discussed in this work. The initial software solution was only able to display the raw I, Q, and $\mathrm{P}$ values and did not include any additional processing, calibration, or graphing capabilities. In addition, viewing the raw data in a terminal is not ideal in a surgical environment. To overcome these challenges, a custom application was created 
in National Instruments LabView to further process, log, and display the implant data. Complete protocol setup, reading data transferred from the implant, writing data to the implant, and finally some limitations with the initial version of software were discussed in this thesis.

- The PV system designed and characterized in this thesis was evaluated in-vivo using a pig. The implant study was performed at Western University Veterinary Services by a trained surgical and technical team. The medical procedure and monitoring was performed in accordance with the approved ethics protocol AUP Number: 2014-003. The study was designed to evaluate the PV system in a non-anaesthetised test subject, i.e., freely moving animal. In addition, the study was completed to determine initial catheterheart interaction. In-vivo results were presented that demonstrate the validity of the RF pressure-volume telemetry system. The PV sensor was implanted into a pig's left ventricle while the encapsulated system was secured in the neck region. Live data was captured in both an acute and a freely moving setting over a 24 hour period. Another goal of this study was to determine the effect that the implant had on the surrounding tissue as well as the effects of the catheter in the left ventricle and surrounding areas. A macroscopic observation of the tissue was completed following euthanasia, and a full histopathology study was completed on select areas of tissue. The results of the histopathology study indicated that lesions were found on the heart and carotid artery. These lesions may have been caused by the catheter insertion and also by the tip of the electrode that broke while insertion. Furthermore, the forces of the LV caused further damage to incur on the catheter tip. To prevent catheter failure, better mating of the introducer and the outer diameter of the pigtail is recommended. Further improvements in catheter design as well as improvements in the implantation methodology will improve the data quality and safety of the subject.

This thesis presented the complete development of a fully implantable pressure-volume measurement telemetry system. All aspects of the design were presented culminating in an 
in-vivo study. In its present form, the telemetry system has a total volume of $2.475 \mathrm{~cm}^{3}$ with a weight of $4.0 \mathrm{~g}$ and is suitable for commercial application in medium sized subjects (large rats, rabbits, pigs, etc). The designed system is $58 \%$ smaller in volume, $44 \%$ in weight and has a $55 \%$ reduction in sampling power over the last reported research in PV telemetry [25, 26]. The achievements in this work advances the field of implantable medical devices forward with the intention to be eventually used Safety Pharmacology drug studies to detect real-time changes in contractility using PV loop analysis. Furthermore, this thesis has created a benchmark to enable further advancement of micro-wireless telemetry systems through the use of a variety of sensors, not limited to cardiac monitoring, as well as allowing for future benefits in eHealth monitoring systems for humans.

\subsection{Future Work}

- Additional in-vivo studies should be undertaken to investigate the full potential of this system. These studies should include multiple animal subjects (large and small) to study the animal to animal variability in free moving animals as well as a longer term study i.e., 1-3 months (protocol dependent). In addition, the full effect of catheter-heart interaction should be studied.

- To achieve long-term in-vivo studies, an updated intermediate implant overcoming the limitations of the existing system should be developed and optimized for these types of studies. Application software should also be improved for the additional studies. Initial designs of this implant have been undertaken and include the following design changes: lower transmission frequency of $915 \mathrm{MHz}$ to reduce power consumption, improved temperature compensation to mitigate possible drift within the animal, an improved magnetic activation switch to reduce startup errors, and improved calibration methods to increase the accuracy of measurements. The current implementation of this longer-term implant is designed on a single PCB and is shown in Fig. 7.1. The physical prototype is de- 


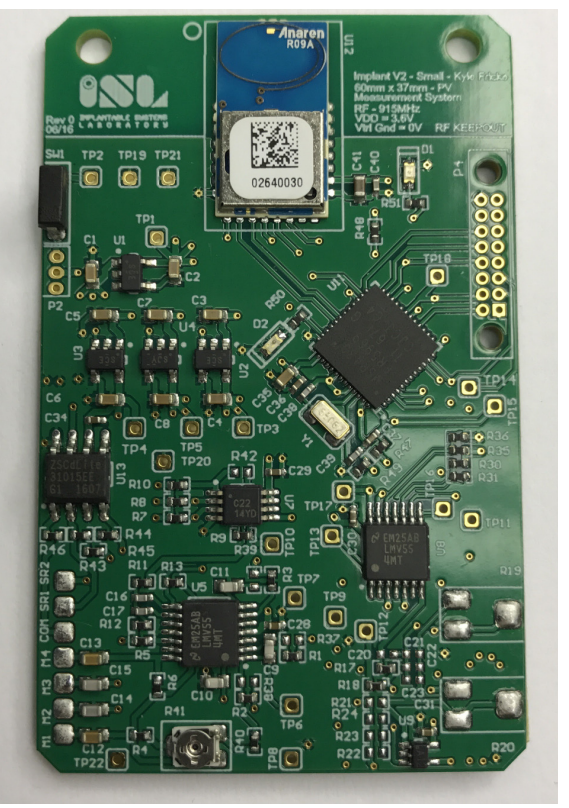

Figure 7.1: Improved version of PV telemetry system designed on a single PCB optimized for long-term studies.

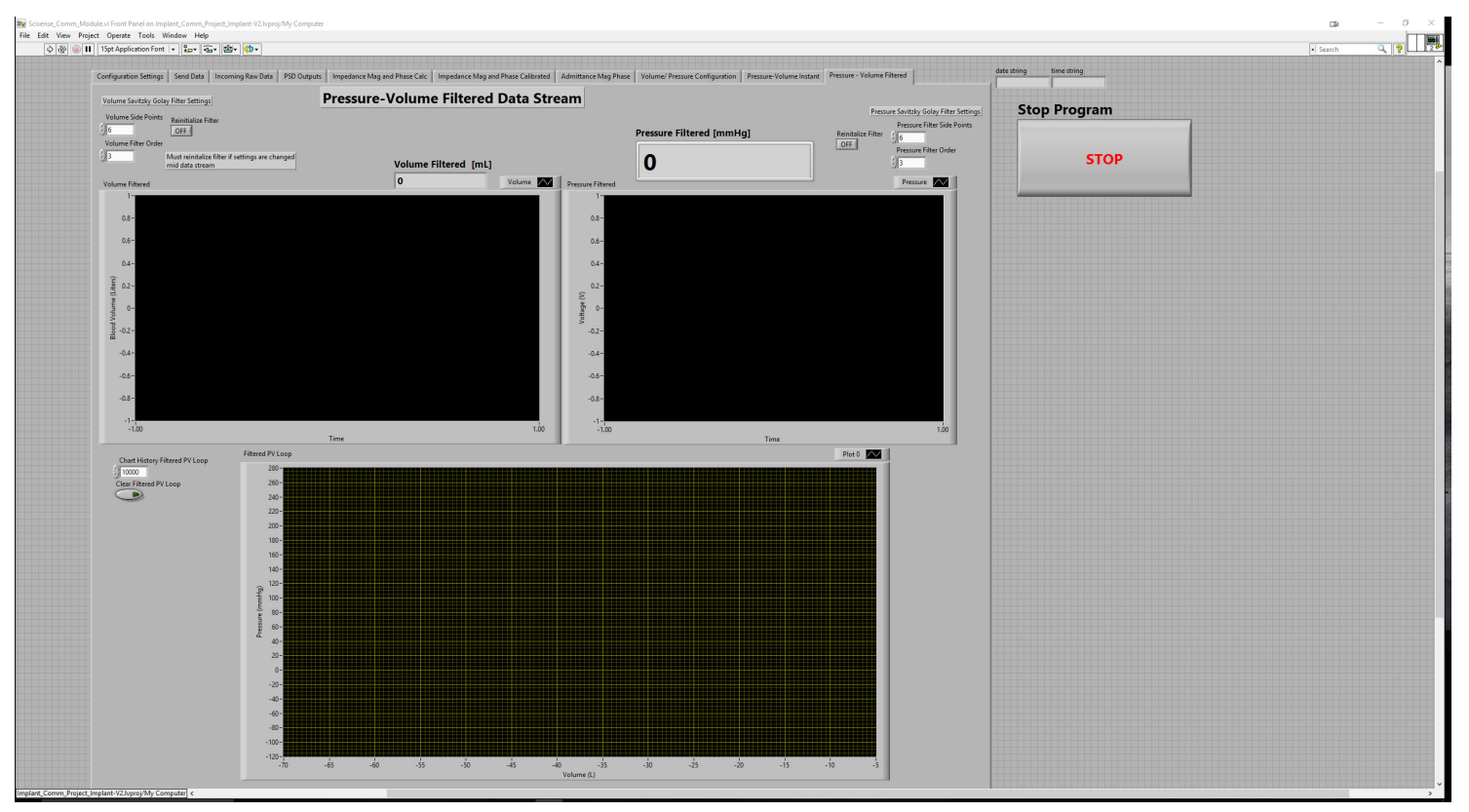

Figure 7.2: Improve LabView measurement application interface. 
signed around a much larger battery to facilitate the long-term tests, requiring a larger enclosure to be designed. This enclosure contains improvements such as visible antenna orientation markers. In addition, an updated labView application has been created that includes built in calibration and volume conversion as well as an improved user interface, Fig. 7.2. This version, following long-term tests, should be reduced down in size including all improvements to achieve a volume similar to the one outlined in this thesis.

- Application measurement software should be developed outside of LabView to ensure a more professional experience. For example, one could develop the application in Java, $\mathrm{C}++$, or Qt. This would remove delays and graphical issues found in LabView software.

- A wireless power solution should be developed to remove the battery. The first implementation would be to use inductive wireless power transfer. Future versions would utilize a full cage solution based on Helmholtz coils and magnetic resonance energy transfer, currently being explored by other members of this research group [136]. Furthermore, design and testing of custom power management circuits (LDO, Bandgap, and Rectifier) are also underway [130,271], which will be fully integrated with a wireless power solution, Fig. 7.3 .

- Future hybrid implant capsules should be designed. This would include Titanium and plastic to create an RF integrated implant package solution. Furthermore, different materials should be explored to determine optimal mechanical properties for an implantable device.

- A custom integrated circuit of the RF transceiver should be developed. The power consumption will be optimized specifically for implantable devices, further reducing the systems overall power consumption. Additionally, an RF startup switch should be developed to reduce power consumption and ensure that the implant is not activated until required by the user. This switch would be integrated into a full RF front-end solution. Initial work on the RF switch has been published in [272]. 


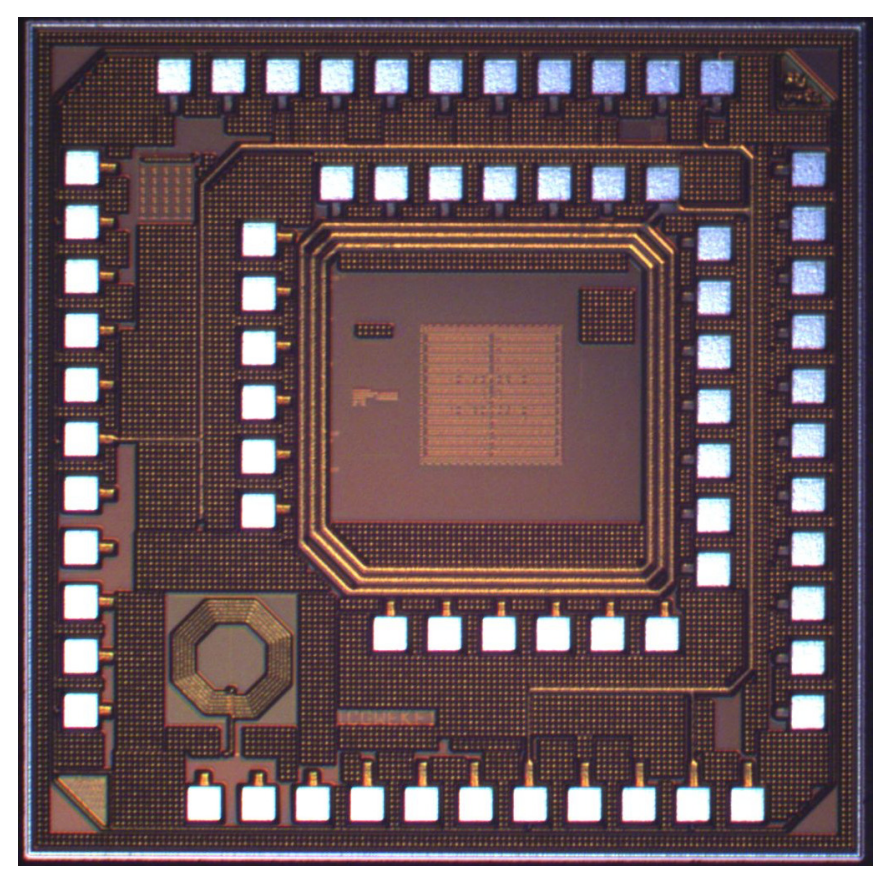

Figure 7.3: Custom test IC with Wireless power sub-blocks and ADC test blocks.

- The microcontroller should be replaced with a custom low power application specific integrated circuit. This processor should be designed only to perform the tasks needed by this application, allowing power consumption to be optimized specifically for the lowpower requirements of this implantable system. Initial work on this custom controller has been published in [273].

- A integrated circuit (IC) of the sensor interface should be developed and tested. This would greatly reduce the size and weight of the implant enabling further miniaturization of the complete system. This IC should be developed in conjunction with the custom controller IC. Additional blocks should be created including analog-to-digital converters(ADC) and other interfaces. A low-power 9-bit ADC has been developed in collaboration with The University of Zagreb that has been fabricated and tested, Fig. 7.3, Results have been published in [274]. This serves as an initial building block in the development of a custom sensor interface IC.

- Finally, all subsystems should be integrated together using state-of-the-art semiconduc- 
tor and packaging technologies. This would enable significant reductions in weight and volume over the implant presented in this thesis. This device would only contain a single IC along with an antenna and sensor connections, thus enabling the encapsulation of the system into a single biocompatible implantable package. The culmination of these advancements would enable a long-term data monitoring solution for small animals in Safety Pharmacology drug testing. 


\section{Bibliography}

[1] Wikipedia, "Wiggers diagram," Wikipedia, Tech. Rep., 2016. [Online]. Available: https://en.wikipedia.org/wiki/Wiggers_diagram

[2] J. R. Mitchell and J.-J. Wang, "Expanding application of the wiggers diagram to teach cardiovascular physiology," Advances in physiology education, vol. 38, no. 2, pp. 170$175,2014$.

[3] T. S. Inc., "Transonic scisense advantage adv500 pv control unit," Transonic Scisense Inc., Tech. Rep., 2016. [Online]. Available: http://www.transonic.com/product/ adv500-pv-system/

[4] R. Sobot, "Implantable rf telemetry for cardiac monitoring in the murine heart: a tutorial review," EURASIP Journal on Embedded Systems, vol. 2013, no. DOI: 10.1186/16873963-2013-1, 2013.

[5] K. G. Fricke, "Wireless telemetry system for implantable sensors," Master's thesis, The University of Western Ontario, 2012.

[6] E. Greenwald, M. Mollazadeh, C. Hu, W. Tang, E. Culurciello, and N. Thakor, "A vlsi neural monitoring system with ultra-wideband telemetry for awake behaving subjects," IEEE transactions on biomedical circuits and systems, vol. 5, no. 2, pp. 112-119, 2011.

[7] G. Gagnon-Turcotte, Y. LeChasseur, C. Bories, Y. Messaddeq, Y. De Koninck, and B. Gosselin, "A wireless headstage for combined optogenetics and multichannel elec- 
trophysiological recording," IEEE Transactions on Biomedical Circuits and Systems, 2016.

[8] X. Liu, M. Zhang, T. Xiong, A. G. Richardson, T. H. Lucas, P. S. Chin, R. EtienneCummings, T. D. Tran, and J. V. der Spiegel, "A fully integrated wireless compressed sensing neural signal acquisition system for chronic recording and brain machine interface," IEEE Transactions on Biomedical Circuits and Systems, vol. 10, no. 4, pp. 874-883, Aug 2016.

[9] M. Azin, D. J. Guggenmos, S. Barbay, R. J. Nudo, and P. Mohseni, "A miniaturized system for spike-triggered intracortical microstimulation in an ambulatory rat," IEEE Transactions on Biomedical Engineering, vol. 58, no. 9, pp. 2589-2597, 2011.

[10] P. Herrero, M. E. Sharkawy, P. Pesl, M. Reddy, N. Oliver, D. Johnston, C. Toumazou, and P. Georgiou, "Live demonstration: A handheld bio-inspired artificial pancreas for treatment of diabetes," in 2014 IEEE Biomedical Circuits and Systems Conference (BioCAS) Proceedings, Oct 2014, pp. 172-172.

[11] C. Baj-Rossi, A. Cavallini, E. G. Kilinc, F. Stradolini, T. R. Jost, M. Proietti, G. De Micheli, F. Grassi, C. Dehollain, and S. Carrara, "In-vivo validation of fully implantable multi-panel devices for remote monitoring of metabolism," IEEE Transactions on Biomedical Circuits and Systems, 2016.

[12] S.-Y. Lee, M. Y. Su, M.-C. Liang, Y.-Y. Chen, C.-H. Hsieh, C.-M. Yang, H.-Y. Lai, J.W. Lin, and Q. Fang, “A programmable implantable microstimulator soc with wireless telemetry: Application in closed-loop endocardial stimulation for cardiac pacemaker," IEEE transactions on biomedical circuits and systems, vol. 5, no. 6, pp. 511-522, 2011.

[13] J. B. van Gaalen, A. L. Trejos, H. N. Nikolov, T. G. Ivanov, S. I. Pollman, and D. W. Holdsworth, "Versatile smart hip implant technology using 3d metal printing," in 2016 
IEEE International Symposium on Circuits and Systems (ISCAS), May 2016, pp. 27312734.

[14] B. P. Brockway, P. A. Mills, and J. T. Miller, "Device for chronic measurement of internal body pressure,’ July 11 1989, uS Patent 4,846,191.

[15] R. D. Sarazan and K. T. Schweitz, "Standing on the shoulders of giants: Dean franklin and his remarkable contributions to physiological measurements in animals," Advances in physiology education, vol. 33, no. 3, pp. 144-156, 2009.

[16] M. I. Inc., “Trm54p pressure telemeter," Millar Instruments Inc., Tech. Rep., 2016. [Online]. Available: http://millar.com/products/telemetry/telemeters/ TRM54P-pressure-telemeter

[17] T. S. Inc., "Endogear 1 telemetry system,” Transonic Scisense Inc., Tech. Rep., 2016. [Online]. Available: http://www.transonic.com/product/endogear1/

[18] —_, "Endogear 3 telemetry system," Transonic Scisense Inc., Tech. Rep., 2016. [Online]. Available: http://www.transonic.com/product/endogear3/

[19] D. S. I. (DSI), "Hd-s21 dual-pressure, single biopotential implant," Data Science International (DSI), Tech. Rep., 2016. [Online]. Available: http://www.datasci.com/ products/implantable-telemetry/small-animal/hd-s21

[20] —_, "Physiotel digital 1 series," Data Science International (DSI), Tech. Rep., 2016. [Online]. Available: http://www.datasci.com/products/implantable-telemetry/ large-animal/physiotel-digital-1

[21] P. Cong, W. Ko, and D. Young, "Wireless batteryless implantable blood pressure monitoring microsystem for small laboratory animals," Sensors Journal, IEEE, vol. 10, no. 2, pp. 243-254, 2010. 
[22] T. Volk, S. Gorbey, M. Bhattacharyya, W. Gruenwald, B. Lemmer, L. M. Reindl, T. Stieglitz, and D. Jansen, "Rfid technology for continuous monitoring of physiological signals in small animals," Biomedical Engineering, IEEE Transactions on, vol. 62, no. 2, pp. 618-626, 2015.

[23] K. Raghavan, M. Feldman, J. Porterfield, E. Larson, J. Jenkins, D. Escobedo, J. Pearce, and J. Valvano, "A bio-telemetric device for measurement of left ventricular pressurevolume loops using the admittance technique in conscious, ambulatory rats," Physiological Measurement, vol. 32, p. 701, 2011.

[24] L. M. Shuhatovich, "Design and implementation of a low-power implantable cardiac monitoring device," Master's thesis, The University of Texas at Austin, 2010.

[25] K. Loeffler, J. E. Porterfield, E. R. Larson, D. Escobedo, G. P. Escobar, M. Feldman, and J. Valvano, "Embedded medical devices: Pressure volume loops in rodents," Potentials, IEEE, vol. 32, no. 1, pp. 18-22, 2013.

[26] K. R. Loeffler, "Development of an implantable system to measure the pressure-volume relationship in ambulatory rodent hearts," Master's thesis, The University of Texas at Austin, 2012.

[27] L. Holt, "Implantable intracardiac bioimpedance system," Master's thesis, The University of Texas at Austin, 2014.

[28] ZMDI, “Zmdi zsc 31015 datasheet,” ZMDI, Tech. Rep., 2016. [Online]. Available: http://www.idt.com/document/dst/zsc31015-datasheet

[29] T. Instruments, “Texas instruments ez430-rf2500," Texas Instruments, Tech. Rep., 2016. [Online]. Available: http://www.ti.com/tool/ez430-rf2500 
[30] K. Fricke, A. Dounavis, and R. Sobot, "Wireless telemetry system for implantable cardiac monitoring in small animal subjects using pressure-volume sensors," in IEEE 11th International NEWCAS 2013 Conference, June 16-19 2013, Paris, France, 2013.

[31] K. Fricke and R. Sobot, "Miniature implantable telemetry system for pressure-volume cardiac monitoring," in Biomedical Circuits and Systems Conference (BioCAS), 2013 IEEE. IEEE, 2013, pp. 282-285.

[32] K. Ito, K. Furuya, Y. Okano, and L. Hamada, "Development and characteristics of a biological tissue-equivalent phantom for microwaves," Electronics and Communications in Japan (Part I: Communications), vol. 84, no. 4, pp. 67-77, 2001.

[33] S. Kutty, A. T. Kottam, A. Padiyath, K. R. Bidasee, L. Li, S. Gao, J. Wu, J. Lof, D. A. Danford, and T. Kuehne, "Validation of admittance computed left ventricular volumes against real-time three-dimensional echocardiography in the porcine heart," Experimental physiology, vol. 98, no. 6, pp. 1092-1101, 2013.

[34] R. D. Sarazan, "Cardiovascular pressure measurement in safety assessment studies: technology requirements and potential errors," Journal of pharmacological and toxicological methods, vol. 70, no. 3, pp. 210-223, 2014.

[35] T. S. Inc., "Cardiac hemodynamic assessment using pressure volume (pv) loops in safety pharmacology," Transonic Scisense Inc., Tech. Rep., 2016. [Online]. Available: http://www.transonic.com/resources/research/ hemodynamic-measurement-of-safety-pharmacology/

[36] B. P. Monahan, C. L. Ferguson, E. S. Killeavy, B. K. Lloyd, J. Troy, and L. R. Cantilena, “Torsades de pointes occurring in association with terfenadine use," Jama, vol. 264, no. 21 , pp. $2788-2790,1990$.

[37] J. Baan, E. Van Der Velde, H. De Bruin, G. Smeenk, J. Koops, A. Van Dijk, D. Temmerman, J. Senden, and B. Buis, "Continuous measurement of left ventricular volume in 
animals and humans by conductance catheter," Circulation, vol. 70, no. 5, pp. 812-823, 1984.

[38] J. Francis, R. Weiss, S. Wei, A. Johnson, and R. Felder, "Progression of heart failure after myocardial infarction in the rat," American Journal of Physiology-Regulatory, Integrative and Comparative Physiology, vol. 281, no. 5, pp. R1734-R1745, 2001.

[39] A. Katz, Physiology of the Heart. Lippincott Williams \& Wilkins, 2010.

[40] P. Nardinocchi, L. Teresi, and V. Varano, "Myocardial contractions and the ventricular pressure-volume relationship," arXiv preprint arXiv:1005.5292, 2010.

[41] D. Burkhoff, I. Mirsky, and H. Suga, "Assessment of systolic and diastolic ventricular properties via pressure-volume analysis: a guide for clinical, translational, and basic researchers," American Journal of Physiology-Heart and Circulatory Physiology, vol. 289, no. 2, pp. H501-H512, 2005.

[42] R. Klabunde, Cardiovascular physiology concepts. Lippincott Williams \& Wilkins, 2011.

[43] Wikipedia, "Pressure-volume loop analysis in cardiology," Wikipedia, Tech. Rep., 2016. [Online]. Available: https://en.wikipedia.org/wiki/Pressure-volume_loop_ analysis_in_cardiology

[44] J. M. BROWN, "Anesthesia and the contractile force of the heart: A review." Anesthesia $\mathcal{E}$ Analgesia, vol. 39, no. 6, pp. 487-498, 1960.

[45] N. Buckley, Z. Penefsky, and R. Litwak, "Comparative force-frequency relationships in human and other mammalian ventricular myocardium," Pflügers Archiv, vol. 332, no. 4, pp. 259-270, 1972.

[46] K. Sagawa, "The ventricular pressure-volume diagram revisited.” Circulation Research, vol. 43 , no. 5, pp. 677-687, 1978. 
[47] R. L. Van Citters, D. L. Franklin, and R. F. Rushmer, "Left ventricular dynamics in dogs during anesthesia with alpha-chloralose and sodium pentobarbital," The american journal of cardiology, vol. 13, no. 3, pp. 349-354, 1964.

[48] L. A. Geddes, Handbook of blood pressure measurement. Springer Science \& Business Media, 1991.

[49] M. Krenz, "Conductance, admittance, and hypertonic saline: should we take ventricular volume measurements with a grain of salt?" Journal of Applied Physiology, vol. 107, no. 6, pp. 1683-1684, 2009.

[50] D. Kass, T. Yamazaki, D. Burkhoff, W. Maughan, and K. Sagawa, "Determination of left ventricular end-systolic pressure-volume relationships by the conductance (volume) catheter technique." Circulation, vol. 73, no. 3, pp. 586-595, 1986.

[51] B. Hoit, S. Kiatchoosakun, J. Restivo, D. Kirkpatrick, K. Olszens, H. Shao, Y. Pao, and J. Nadeau, "Naturally occurring variation in cardiovascular traits among inbred mouse strains," Genomics, vol. 79, no. 5, pp. 679-685, 2002.

[52] K. Sagawa, H. Suga, and W. Maugham, Cardiac contraction and the pressure-volume relationship. Oxford University Press New York:, 1988, vol. 480.

[53] H. Haber, C. Simek, L. Gimple, J. Bergin, K. Subbiah, A. Jayaweera, E. Powers, and M. Feldman, "Why do patients with congestive heart failure tolerate the initiation of beta-blocker therapy?" Circulation, vol. 88, no. 4, pp. 1610-1619, 1993.

[54] H. Haber, E. Powers, L. Gimple, C. Wu, K. Subbiah, W. Johnson, and M. Feldman, "Intracoronary angiotensin-converting enzyme inhibition improves diastolic function in patients with hypertensive left ventricular hypertrophy," Circulation, vol. 89, no. 6, pp. 2616-2625, 1994. 
[55] M. Feldman, P. Pak, C. Wu, H. Haber, C. Heesch, J. Bergin, E. Powers, T. Cowart, W. Johnson, A. Feldman, et al., "Acute cardiovascular effects of opc-18790 in patients with congestive heart failure: time-and dose-dependence analysis based on pressurevolume relations," Circulation, vol. 93, no. 3, pp. 474-483, 1996.

[56] T. S. Inc., "Understanding contractility: Cardiac intropy," Transonic Scisense Inc., Tech. Rep., 2016. [Online]. Available: http://www.transonic.com/resources/research/ understanding-contractility-cardiac-intropy/

[57] D. S. I. (DSI), "Left ventricular pressure," Data Science International (DSI), Tech. Rep., 2016. [Online]. Available: https://www.datasci.com/solutions/cardiovascular/ left-ventricular-pressure

[58] U. S. Food and D. A. (FDA), "S7a safety pharmacology studies for human pharmaceuticals - fda," United States Food and Drug Administration (FDA), Tech. Rep., 2016. [Online]. Available: http://www.fda.gov/downloads/ Drugs/GuidanceComplianceRegulatoryInformation/Guidances/UCM074959.pdf

[59] I. C. on Harmonisation (ICH), "S7a safety pharmacology studies for human pharmaceuticals," International Conference on Harmonisation (ICH), Tech. Rep. [Online]. Available: http://www.ich.org/products/guidelines/safety/safety-single/article/ safety-pharmacology-studies-for-human-pharmaceuticals.html

[60] U. S. Food and D. A. (FDA), "S7b nonclinical evaluation of the potential for delayed ventricular repolarization (qt interval prolongation) by human pharmaceuticals," United States Food and Drug Administration (FDA), Tech. Rep., 2016. [Online]. Available: http://www.fda.gov/downloads/drugs/guidancecomplianceregulatoryinformation/ guidances/ucm074963.pdf

[61] I. C. on Harmonisation (ICH), "S7b nonclinical evaluation of the potential for delayed ventricular repolarization (qt interval prolongation) by 
human pharmaceuticals," International Conference on Harmonisation (ICH), Tech. Rep., 2016. [Online]. Available: http://www.ich.org/products/guidelines/ safety/safety-single/article/the-non-clinical-evaluation-of-the-potential-for-delayed/ -ventricular-repolarization-qt-interval-pro.html

[62] L. Mooney, L. Marks, K. L. Philp, M. Skinner, S. J. Coker, and S. Currie, “Optimising conditions for studying the acute effects of drugs on indices of cardiac contractility and on haemodynamics in anaesthetized guinea pigs," Journal of pharmacological and toxicological methods, vol. 66, no. 1, pp. 43-51, 2012.

[63] D. M. Johnson, R. Geys, J. Lissens, and P. Guns, "Drug-induced effects on cardiovascular function in pentobarbital anesthetized guinea-pigs: Invasive lvp measurements versus the qa interval," Journal of pharmacological and toxicological methods, vol. 66, no. 2, pp. 152-159, 2012.

[64] K. Norton, G. Iacono, and M. Vezina, "Assessment of the pharmacological effects of inotropic drugs on left ventricular pressure and contractility: an evaluation of the qa interval as an indirect indicator of cardiac inotropism," Journal of pharmacological and toxicological methods, vol. 60, no. 2, pp. 193-197, 2009.

[65] M. Markert, T. Trautmann, M. Groß, A. Ege, K. Mayer, and B. Guth, "Evaluation of a method to correct the contractility index lvdp/dt max for changes in heart rate," Journal of pharmacological and toxicological methods, vol. 66, no. 2, pp. 98-105, 2012.

[66] T. Bombardini, "Myocardial contractility in the echo lab: molecular, cellular and pathophysiological basis," Cardiovascular ultrasound, vol. 3, no. 1, p. 1, 2005.

[67] L. Marks, S. Borland, K. Philp, L. Ewart, P. Lainée, M. Skinner, S. Kirk, and J.-P. Valentin, "The role of the anaesthetised guinea-pig in the preclinical cardiac safety evaluation of drug candidate compounds," Toxicology and applied pharmacology, vol. 263, no. 2 , pp. 171-183, 2012. 
[68] J. D. Violin, S. M. DeWire, D. Yamashita, D. H. Rominger, L. Nguyen, K. Schiller, E. J. Whalen, M. Gowen, and M. W. Lark, "Selectively engaging $\beta$-arrestins at the angiotensin ii type 1 receptor reduces blood pressure and increases cardiac performance," Journal of Pharmacology and Experimental Therapeutics, vol. 335, no. 3, pp. 572-579, 2010.

[69] J. James, T. Hewett, and J. Robbins, “Cardiac physiology in transgenic mice," Circulation research, vol. 82, no. 4, pp. 407-415, 1998.

[70] M. Feldman, J. Erikson, Y. Mao, C. Korcarz, R. Lang, and G. Freeman, "Validation of a mouse conductance system to determine lv volume: comparison to echocardiography and crystals," American Journal of Physiology-Heart and Circulatory Physiology, vol. 279, no. 4, pp. H1698-H1707, 2000.

[71] Y. Zhou, F. Foster, B. Nieman, L. Davidson, X. Chen, and R. Henkelman, “Comprehensive transthoracic cardiac imaging in mice using ultrasound biomicroscopy with anatomical confirmation by magnetic resonance imaging," Physiological genomics, vol. 18, no. 2, pp. 232-244, 2004.

[72] M. Drangova, N. Ford, S. Detombe, A. Wheatley, and D. Holdsworth, "Fast retrospectively gated quantitative four-dimensional (4d) cardiac micro computed tomography imaging of free-breathing mice," Investigative radiology, vol. 42, no. 2, pp. 85-94, 2007.

[73] C. Jacoby, A. Molojavyi, U. Flögel, M. Merx, Z. Ding, and J. Schrader, "Direct comparison of magnetic resonance imaging and conductance microcatheter in the evaluation of left ventricular function in mice," Basic research in cardiology, vol. 101, no. 1, pp. 87-95, 2006.

[74] E. Winter, R. Grauss, D. Atsma, B. Hogers, R. Poelmann, R. Van Der Geest, C. Tschöpe, M. Schalij, A. Gittenberger-de Groot, and P. Steendijk, "Left ventricular function in 
the post-infarct failing mouse heart by magnetic resonance imaging and conductance catheter: a comparative analysis," Acta Physiologica, vol. 194, no. 2, pp. 111-122, 2008.

[75] F. Franco, S. Dubois, R. Peshock, and R. Shohet, "Magnetic resonance imaging accurately estimates lv mass in a transgenic mouse model of cardiac hypertrophy," American Journal of Physiology-Heart and Circulatory Physiology, vol. 274, no. 2, pp. H679H683, 1998.

[76] G. Esposito, L. Santana, K. Dilly, J. Cruz, L. Mao, W. Lederer, and H. Rockman, “Cellular and functional defects in a mouse model of heart failure," American Journal of Physiology-Heart and Circulatory Physiology, vol. 279, no. 6, pp. H3101-H3112, 2000.

[77] J. Baan, T. Jong, P. Kerkhof, R. Moene, A. D Van Dijk, E. T Van Der Velde, and J. Koops, "Continuous stroke volume and cardiac output from intra-ventricular dimensions obtained with impedance catheter," Cardiovascular Research, vol. 15, no. 6, pp. 328-334, 1981.

[78] A. Kottam, J. Porterfield, K. Raghavan, D. Fernandez, M. Feldman, J. Valvano, and J. Pearce, "Real time pressure-volume loops in mice using complex admittance: measurement and implications," in Engineering in Medicine and Biology Society, 2006. EMBS'06. 28th Annual International Conference of the IEEE. IEEE, 2006, pp. 43364339.

[79] P. Cong, N. Chaimanonart, W. Ko, and D. Young, "A wireless and batteryless 10-bit implantable blood pressure sensing microsystem with adaptive rf powering for real-time laboratory mice monitoring," Solid-State Circuits, IEEE Journal of, vol. 44, no. 12, pp. 3631-3644, 2009.

[80] A. Kottam, J. Dubois, A. McElligott, and K. K. Henderson, "Novel approach to admittance to volume conversion for ventricular volume measurement," in Engineering 
in Medicine and Biology Society, EMBC, 2011 Annual International Conference of the IEEE. IEEE, 2011, pp. 2514-2517.

[81] Y. Gao, Y. Zheng, S. Diao, W. Toh, C. Ang, M. Je, and C. Heng, "Low-power ultrawideband wireless telemetry transceiver for medical sensor applications," Biomedical Engineering, IEEE Transactions on, vol. 58, no. 3, pp. 768-772, 2011.

[82] P. Dario, M. Carrozza, A. Benvenuto, and A. Menciassi, "Micro-systems in biomedical applications," Journal of Micromechanics and Microengineering, vol. 10, no. 2, p. 235, 2000.

[83] S. Lee, H. Lee, M. Kiani, U. Jow, and M. Ghovanloo, "An inductively powered scalable 32-channel wireless neural recording system-on-a-chip for neuroscience applications," Biomedical Circuits and Systems, IEEE Transactions on, vol. 4, no. 6, pp. 360-371, 2010.

[84] R. Häusler, C. Stieger, H. Bernhard, and M. Kompis, "A novel implantable hearing system with direct acoustic cochlear stimulation," Audiology and Neurotology, vol. 13, no. 4, pp. 247-256, 2008.

[85] E. Chow, A. Chlebowski, S. Chakraborty, W. Chappell, and P. Irazoqui, "Fully wireless implantable cardiovascular pressure monitor integrated with a medical stent," Biomedical Engineering, IEEE Transactions on, vol. 57, no. 6, pp. 1487-1496, 2010.

[86] N. Neihart and R. Harrison, "Micropower circuits for bidirectional wireless telemetry in neural recording applications," Biomedical Engineering, IEEE Transactions on, vol. 52, no. 11, pp. 1950-1959, 2005.

[87] Y. Shih, T. Shen, and B. Otis, "A 2.3 $\mu \mathrm{w}$ wireless intraocular pressure/temperature monitor," in Solid State Circuits Conference (A-SSCC), 2010 IEEE Asian. IEEE, 2010, pp. 1-4. 
[88] C. Baj-Rossi, E. Kilinc, S. Ghoreishizadeh, D. Casarino, T. Jost, C. Dehollain, F. Grassi, L. Pastorino, G. De Micheli, and S. Carrara, "Fabrication and packaging of a fully implantable biosensor array," in Biomedical Circuits and Systems Conference (BioCAS), 2013 IEEE, Oct 2013, pp. 166-169.

[89] N. J. Cleven, P. Isfort, T. Penzkofer, A. Woitok, B. Hermanns-Sachweh, U. Steinseifer, and T. Schmitz-Rode, "Wireless blood pressure monitoring with a novel implantable device: long-term in vivo results," Cardiovascular and interventional radiology, vol. 37, no. 6, pp. 1580-1588, 2014.

[90] K. Uemura, T. Kawada, M. Sugimachi, C. Zheng, K. Kashihara, T. Sato, and K. Sunagawa, "A self-calibrating telemetry system for measurement of ventricular pressurevolume relations in conscious, freely moving rats," American Journal of PhysiologyHeart and Circulatory Physiology, vol. 287, no. 6, pp. H2906-H2913, 2004.

[91] R. Receveur, F. Lindemans, and N. de Rooij, "Microsystem technologies for implantable applications," Journal of Micromechanics and Microengineering, vol. 17, no. 5, p. R50, 2007.

[92] T. Stieglitz, "Implantable microsystems for monitoring and neural rehabilitation, part i," Med Device Technol, vol. 12, pp. 16-18, 2001.

[93] W. Mokwa and U. Schnakenberg, "On-chip microsystems for medical applications," in Proc. Microsystem Symp.(Delft), 1998, pp. 69-75.

[94] Y.-C. Shih, T. Shen, and B. P. Otis, “A $2.3 \mu \mathrm{w}$ wireless intraocular pressure/temperature monitor," Solid-State Circuits, IEEE Journal of, vol. 46, no. 11, pp. 2592-2601, 2011.

[95] J. Goosen, D. Tanase, and P. French, "Silicon sensors for use in catheters," in Microtechnologies in Medicine and Biology, 1st Annual International, Conference On. 2000. IEEE, 2000, pp. 152-155. 
[96] A. Schneider, T. Stieglitz, W. Haberer, H. Beutel, and J. Meyer, "Flexible interconnects for biomedical microsystems assembly," in 34th Int. Symp. Microelectronics IMAPS, Baltimore, MD, 2001.

[97] A. Sivard, P. Bradley, P. Chadwick, and H. Higgins, “The challenge of designing in-body communications," Embedded Systems Programming, vol. 17, no. 11, pp. 20-28, 2004.

[98] Z. Yang, W. Liu, and E. Basham, "Inductor modeling in wireless links for implantable electronics," Magnetics, IEEE Transactions on, vol. 43, no. 10, pp. 3851-3860, 2007.

[99] M. Ghovanloo and K. Najafi, "A wireless implantable multichannel microstimulating system-on-a-chip with modular architecture," Neural Systems and Rehabilitation Engineering, IEEE Transactions on, vol. 15, no. 3, pp. 449-457, 2007.

[100] D. Zhang, Y. Dong, M. Li, and H. Wang, "A radio-telemetry system for navigation and recording neuronal activity in free-roaming rats," Journal of Bionic Engineering, vol. 9, no. 4, pp. 402-410, 2012.

[101] A. Khalifa, J. Zhang, M. Leistner, and R. Etienne-Cummings, "A compact, low-power, fully analog implantable microstimulator,' in 2016 IEEE International Symposium on Circuits and Systems (ISCAS), May 2016, pp. 2435-2438.

[102] H. Bahrami, S. A. Mirbozorgi, L. A. Rusch, and B. Gosselin, "Biological channel modeling and implantable uwb antenna design for neural recording systems," IEEE Transactions on Biomedical Engineering, vol. 62, no. 1, pp. 88-98, 2015.

[103] C. Zuo, X. Yang, Y. Wang, C. E. Hagains, A.-L. Li, Y. B. Peng, and J. Chiao, "A digital wireless system for closed-loop inhibition of nociceptive signalsgroups 1, 2 and 3 contributed equally to this project." Journal of neural engineering, vol. 9, no. 5, p. 056010 , 2012. 
[104] D. Fan, D. Rich, T. Holtzman, P. Ruther, J. W. Dalley, A. Lopez, M. A. Rossi, J. W. Barter, D. Salas-Meza, S. Herwik, et al., “A wireless multi-channel recording system for freely behaving mice and rats," PloS one, vol. 6, no. 7, p. e22033, 2011.

[105] M. Sawan, Y. Hu, and J. Coulombe, "Wireless smart implants dedicated to multichannel monitoring and microstimulation," IEEE Circuits and Systems Magazine, vol. 5, no. 1, pp. 21-39, 2005.

[106] M. Ahmadi and G. Jullien, “A wireless-implantable microsystem for continuous blood glucose monitoring," Biomedical Circuits and Systems, IEEE Transactions on, vol. 3, no. 3, pp. 169-180, 2009.

[107] S. Smith, T. Tang, J. Stevenson, B. Flynn, H. Reekie, A. Murray, A. Gundlach, D. Renshaw, B. Dhillon, A. Ohtori, et al., "Miniaturised drug delivery system with wireless power transfer and communication," in MEMS Sensors and Actuators, 2006. The Institution of Engineering and Technology Seminar on. IET, 2006, pp. 155-162.

[108] P. Basappa Khannur, K. L. Chan, J. H. Cheong, K. Kang, A. Astuti Lee, X. Liu, H. J. Lim, K. Ramakrishna, and M. Je, “A $21.6 \mu w$ inductively powered implantable ic for blood flow measurement," in Solid State Circuits Conference (A-SSCC), 2010 IEEE Asian, nov. 2010, pp. $1-4$.

[109] I. Pagkalos, P. Herrero, C. Toumazou, and P. Georgiou, "Bio-inspired glucose control in diabetes based on an analogue implementation of a $\beta$-cell model," IEEE Transactions on Biomedical Circuits and Systems, vol. 8, no. 2, pp. 186-195, April 2014.

[110] R. Turner, R. Holman, C. Cull, I. Stratton, D. Matthews, V. Frighi, S. Manley, A. Neil, K. Mcelroy, D. Wright, et al., "Intensive blood-glucose control with sulphonylureas or insulin compared with conventional treatment and risk of complications in patients with type 2 diabetes (ukpds 33)," lancet, vol. 352, no. 9131, pp. 837-853, 1998. 
[111] S. S. Ghoreishizadeh, C. Baj-Rossi, A. Cavallini, S. Carrara, and G. De Micheli, "An integrated control and readout circuit for implantable multi-target electrochemical biosensing," IEEE transactions on biomedical circuits and systems, vol. 8, no. 6, pp. 891-898, 2014.

[112] C. N. Kotanen, F. G. Moussy, S. Carrara, and A. Guiseppi-Elie, "Implantable enzyme amperometric biosensors," Biosensors and Bioelectronics, vol. 35, no. 1, pp. 14-26, 2012.

[113] J. Olivo, S. Carrara, and G. De Micheli, "Energy harvesting and remote powering for implantable biosensors," IEEE Sensors Journal, vol. 11, no. 7, pp. 1573-1586, 2011.

[114] C. Stagni, C. Guiducci, L. Benini, B. Riccò, S. Carrara, B. Samorí, C. Paulus, M. Schienle, M. Augustyniak, and R. Thewes, "Cmos dna sensor array with integrated a/d conversion based on label-free capacitance measurement," IEEE Journal of SolidState Circuits, vol. 41, no. 12, pp. 2956-2964, 2006.

[115] S. Carrara, S. Ghoreishizadeh, J. Olivo, I. Taurino, C. Baj-Rossi, A. Cavallini, M. Op de Beeck, C. Dehollain, W. Burleson, F. G. Moussy, et al., "Fully integrated biochip platforms for advanced healthcare," Sensors, vol. 12, no. 8, pp. 11 013-11 060, 2012.

[116] W. Yu, R. Rahimi, M. Ochoa, R. Pinal, and B. Ziaie, "A smart capsule with gitract-location-specific payload release," Biomedical Engineering, IEEE Transactions on, vol. 62, no. 9, pp. 2289-2295, 2015.

[117] S. Hached, A. Trigui, O. Loutochin, A. Garon, J. Corcos, and M. Sawan, "Novel electromechanic artificial urinary sphincter," IEEE/ASME Transactions on Mechatronics, vol. 21, no. 2, pp. 945-955, 2016.

[118] A. Kim, C. Powell, and B. Ziaie, "An implantable pressure sensing system with electromechanical interrogation scheme," Biomedical Engineering, IEEE Transactions on, vol. 61, no. 7, pp. 2209-2217, July 2014. 
[119] T. Karacolak, A. Hood, and E. Topsakal, "Design of a dual-band implantable antenna and development of skin mimicking gels for continuous glucose monitoring," $\mathrm{Mi}$ crowave Theory and Techniques, IEEE Transactions on, vol. 56, no. 4, pp. 1001-1008, 2008.

[120] T. Karacolak, R. Cooper, and E. Topsakal, "Electrical properties of rat skin and design of implantable antennas for medical wireless telemetry," Antennas and Propagation, IEEE Transactions on, vol. 57, no. 9, pp. 2806-2812, 2009.

[121] B. Gosselin, M. Sawan, and C. A. Chapman, "A low-power integrated bioamplifier with active low-frequency suppression," IEEE Transactions on Biomedical Circuits and Systems, vol. 1, no. 3, pp. 184-192, 2007.

[122] R. Ritter, J. G. Kauffman, J. Becker, and M. Ortmanns, "A 10 mhz bandwidth, 70 db sndr continuous time delta-sigma modulator with digitally improved reconfigurable blocker rejection,' IEEE Journal of Solid-State Circuits, vol. 51, no. 3, pp. 660-670, March 2016.

[123] M. Baker and R. Sarpeshkar, "Feedback analysis and design of rf power links for lowpower bionic systems," Biomedical Circuits and Systems, IEEE Transactions on, vol. 1, no. 1, pp. 28-38, 2007.

[124] A. Kumar, S. Mirabbasi, and M. Chiao, "Resonance-based wireless power delivery for implantable devices," in Biomedical Circuits and Systems Conference, 2009. BioCAS 2009. IEEE. IEEE, 2009, pp. 25-28.

[125] E. K. Lee, "An inside body power and bidirectional data transfer ic module pair," IEEE Journal of Solid-State Circuits, vol. 46, no. 8, pp. 1820-1831, 2011.

[126] E. Kilinc, M. Ghanad, F. Maloberti, and C. Dehollain, "A remotely powered implantable biomedical system with location detector," Biomedical Circuits and Systems, IEEE Transactions on, vol. PP, no. 99, pp. 1-1, 2014. 
[127] E. G. Kilinc, C. Baj-Rossi, S. Ghoreishizadeh, S. Riario, F. Stradolini, C. Boero, G. De Micheli, F. Maloberti, S. Carrara, and C. Dehollain, "A system for wireless power transfer and data communication of long-term bio-monitoring," IEEE Sensors Journal, vol. 15 , no. 11 , pp. 6559-6569, 2015.

[128] E. G. Kilinc, C. Dehollain, and F. Maloberti, Remote powering and Data Communication for implanted biomedical systems. S Springer, 2016.

[129] H. Xu, J. Handwerker, and M. Ortmanns, “Telemetry for implantable medical devices: Part 2 - power telemetry," IEEE Solid-State Circuits Magazine, vol. 6, no. 3, pp. 60-63, Summer 2014.

[130] K. Fricke, M. Cai, and R. Sobot, "Cmos voltage regulator for rf energy harvester," in IEEE 13th International NEWCAS 2015 Conference, June 7-10 2015, Grenoble, France, 2015.

[131] A. RamRakhyani, S. Mirabbasi, and M. Chiao, "Design and Optimization of ResonanceBased Efficient Wireless Power Delivery Systems for Biomedical Implants,” Biomedical Circuits and Systems, IEEE Transactions on, vol. 5, no. 1, pp. 48-63, 2011.

[132] K. M. Silay, C. Dehollaini, and M. Declercq, "Inductive power link for a wireless cortical implant with two-body packaging," Sensors Journal, IEEE, vol. 11, no. 11, pp. 2825$2833,2011$.

[133] S. Kim, J. S. Ho, and A. S. Poon, "Wireless power transfer to miniature implants: transmitter optimization," Antennas and Propagation, IEEE Transactions on, vol. 60, no. 10, pp. $4838-4845,2012$.

[134] A. Sanni, A. Vilches, and C. Toumazou, "Inductive and ultrasonic multi-tier interface for low-power, deeply implantable medical devices," IEEE transactions on biomedical circuits and systems, vol. 6, no. 4, pp. 297-308, 2012. 
[135] F. Mazzilli, P. E. Thoppay, V. Praplan, and C. Dehollain, "Ultrasound energy harvesting system for deep implanted-medical-devices (imds)," in 2012 IEEE International Symposium on Circuits and Systems. IEEE, 2012, pp. 2865-2868.

[136] S. Senjuti, K. Fricke, A. Dounavis, and R. Sobot, "Misalignment analysis of resonancebased wireless power transfer to biomedical implants," in Electrical Computer Engineering (CCECE), 2012 25th IEEE Canadian Conference on, 29 2012-may 2 2012, pp. $1-5$.

[137] E. G. Kilinc, A. C. Moya, H. Van Lintel, P. Renaud, F. Maloberti, Q. Wang, and C. Dehollain, "Remotely powered implantable heart monitoring system for freely moving animals," in Advances in Sensors and Interfaces (IWASI), 2013 5th IEEE International Workshop on. IEEE, 2013, pp. 10-13.

[138] S. Ayazian and A. Hassibi, "Delivering optical power to subcutaneous implanted devices," in 2011 Annual International Conference of the IEEE Engineering in Medicine and Biology Society. IEEE, 2011, pp. 2874-2877.

[139] A. Zebda, S. Cosnier, J.-P. Alcaraz, M. Holzinger, A. Le Goff, C. Gondran, F. Boucher, F. Giroud, K. Gorgy, H. Lamraoui, et al., "Single glucose biofuel cells implanted in rats power electronic devices," Scientific reports, vol. 3, p. 1516, 2013.

[140] Y. Zhang, F. Zhang, Y. Shakhsheer, J. D. Silver, A. Klinefelter, M. Nagaraju, J. Boley, J. Pandey, A. Shrivastava, E. J. Carlson, et al., "A batteryless 19 w mics/ism-band energy harvesting body sensor node soc for exg applications," IEEE Journal of Solid-State Circuits, vol. 48, no. 1, pp. 199-213, 2013.

[141] S. R. Platt, S. Farritor, K. Garvin, and H. Haider, "The use of piezoelectric ceramics for electric power generation within orthopedic implants," IEEE/ASME Transactions on Mechatronics, vol. 10, no. 4, pp. 455-461, 2005. 
[142] S. J. Medical. (2016) Cardiomems hf system. [Online]. Available: https://www.sjm. com/sitecore/content/professional/therapies/cardiomems/home

[143] M. Rasouli and L. S. J. Phee, "Energy sources and their development for application in medical devices," Expert review of medical devices, vol. 7, no. 5, pp. 693-709, 2010.

[144] S. A. Mirbozorgi, H. Bahrami, M. Sawan, and B. Gosselin, "A smart cage with uniform wireless power distribution in $3 \mathrm{~d}$ for enabling long-term experiments with freely moving animals," IEEE transactions on biomedical circuits and systems, vol. 10, no. 2, pp. 424434, 2016.

[145] B. Lee, M. Kiani, and M. Ghovanloo, "A triple-loop inductive power transmission system for biomedical applications," IEEE transactions on biomedical circuits and systems, vol. 10, no. 1, pp. 138-148, 2016.

[146] D. Ahn and M. Ghovanloo, "Optimal design of wireless power transmission links for millimeter-sized biomedical implants," IEEE transactions on biomedical circuits and systems, vol. 10, no. 1, pp. 125-137, 2016.

[147] C. Gabriel, S. Gabriel, and E. Corthout, "The dielectric properties of biological tissues: I. literature survey," Physics in medicine and biology, vol. 41, p. 2231, 1996.

[148] S. Gabriel, R. Lau, and C. Gabriel, "The dielectric properties of biological tissues: Ii. measurements in the frequency range $10 \mathrm{hz}$ to $20 \mathrm{ghz}$,' Physics in medicine and biology, vol. 41, p. 2251, 1996.

[149] — - "The dielectric properties of biological tissues: Iii. parametric models for the dielectric spectrum of tissues," Physics in medicine and biology, vol. 41, no. 11, p. $2271,1996$. 
[150] R. Ritter, J. Handwerker, T. Liu, and M. Ortmanns, “Telemetry for implantable medical devices: Part 1 - media properties and standards," Solid-State Circuits Magazine, IEEE, vol. 6, no. 2, pp. 47-51, Spring 2014.

[151] F. C. C. (FCC), "Medradioband fcc," Federal Communications Commission (FCC), Tech. Rep., 2015. [Online]. Available: http://www.fcc.gov/encyclopedia/ medical-device-radiocommunications-service-medradio

[152] _ _ "15-radio frequency devices," Federal Communications Commission (FCC), Tech. Rep., 2006. [Online]. Available: http://www.fcc.gov/oet/info/rules/part15/ part15-2-16-06.pdf

[153] M. Asili, R. Green, S. Seran, and E. Topsakal, "A small implantable antenna for medradio and ism bands," IEEE Antennas and Wireless Propagation Letters, no. 11, pp. 1683$1685,2012$.

[154] J. Ung and T. Karacolak, "A wideband implantable antenna for continuous health monitoring in the medradio and ism bands," IEEE Antennas and Wireless Propagation Letters, vol. 11, pp. 1642-1645, 2012.

[155] J. Sametinger, J. Rozenblit, R. Lysecky, and P. Ott, "Security challenges for medical devices," Communications of the ACM, vol. 58, no. 4, pp. 74-82, 2015.

[156] N. Du, N. Manjunath, Y. Shuai, D. Bürger, I. Skorupa, R. Schüffny, C. Mayr, D. N. Basov, M. Di Ventra, O. G. Schmidt, et al., "Novel implementation of memristive systems for data encryption and obfuscation," Journal of Applied Physics, vol. 115, no. 12, p. $124501,2014$.

[157] A. Ascoli, V. Senger, R. Tetzlaff, N. Du, O. G. Schmidt, and H. Schmidt, "Bifeo3 memristor-based encryption of medical data," in 2016 IEEE International Symposium on Circuits and Systems (ISCAS), May 2016, pp. 1602-1605. 
[158] L. Bu, P. Cong, H.-I. Kuo, X. Ye, and W. Ko, "Micro package of short term wireless implantable microfabricated systems," in Engineering in Medicine and Biology Society, 2009. EMBC 2009. Annual International Conference of the IEEE. IEEE, 2009, pp. 6395-6399.

[159] M. L. Scarpello, D. Kurup, H. Rogier, D. Vande Ginste, F. Axisa, J. Vanfleteren, W. Joseph, L. Martens, and G. Vermeeren, "Design of an implantable slot dipole conformal flexible antenna for biomedical applications," Antennas and Propagation, IEEE Transactions on, vol. 59, no. 10, pp. 3556-3564, 2011.

[160] H. Park, M. Kiani, H. M. Lee, J. Kim, J. Block, B. Gosselin, and M. Ghovanloo, “A wireless magnetoresistive sensing system for an intraoral tongue-computer interface," IEEE Transactions on Biomedical Circuits and Systems, vol. 6, no. 6, pp. 571-585, Dec 2012.

[161] T. Broten, S. Kivlighn, C. Harvey, A. Scott, T. Schorn, and P. Siegl, "Techniques for the measurement of arterial blood pressure," Measurement of cardiovascular function, 1997.

[162] C. Caro, The Mechanics of the circulation. Oxford University Press (Oxford and New York), 1978.

[163] W. Ko, J. Hynecek, and S. Boettcher, "Implantable pressure transducer for biomedical applications," in 27th Electronic Component Conf., Arlington, Virginia, May, vol. 16, 1959.

[164] M. K. PARK and W. G. GUNTHEROTH, "Direct blood pressure measurements in brachial and femoral arteries in children," Circulation, vol. 41, no. 2, pp. 231-237, 1970.

[165] A. Ginggen, Y. Tardy, R. Crivelli, T. Bork, and P. Renaud, "A telemetric pressure sensor system for biomedical applications," IEEE Transactions on Biomedical Engineering, vol. 55, no. 4, pp. 1374-1381, 2008. 
[166] K. Ouriel, "Peripheral arterial disease," The lancet, vol. 358, no. 9289, pp. 1257-1264, 2001.

[167] A. H. Association, "What is peripheral vascular disease?" American Heart Association, Tech. Rep., 2015. [Online]. Available: https://www.heart.org/idc/groups/heart-public/ @wcm/@hcm/documents/downloadable/ucm_300323.pdf

[168] D. S. I. (DSI), "Selecting the most appropriate blood pressure measurement method for preclinical research: Aha recommendations then and now,” DSI, Tech. Rep., 2015.

[169] S. Hales, "Statical essays haemostaticks vol. 2," London, W. Innys and Manby, 1738.

[170] W. H. Ko, J. Hynecek, and S. Boettcher, "Development of a miniature pressure transducer for biomedical applications," Electron Devices, IEEE Transactions on, vol. 26, no. 12 , pp. $1896-1905$, dec 1979.

[171] F. W. Casadei, M. Gerold, and E. Baldinger, "Implantable blood pressure telemetry system," IEEE Transactions on Biomedical Engineering, no. 5, pp. 334-341, 1972.

[172] T. W. Kurtz, K. A. Griffin, A. K. Bidani, R. L. Davisson, and J. E. Hall, "Recommendations for blood pressure measurement in humans and experimental animals part 2: blood pressure measurement in experimental animals. a statement for professionals from the subcommittee of professional and public education of the american heart association council on high blood pressure research," Arteriosclerosis, Thrombosis, and Vascular Biology, vol. 25, no. 3, pp. e22-e33, 2005.

[173] E. G. Brennan and N. J. O’Hare, "Calibration and assessment of a fluid-filled cathetertransducer system for the measurement of ventricular diastolic pressures," Physiological measurement, vol. 19, no. 3, p. 405, 1998. 
[174] B. Brockway, P. Mills, and S. Azar, "A new method for continuous chronic measurement and recording of blood pressure, heart rate and activity in the rat via radio-telemetry," Clinical and Experimental Hypertension, vol. 13, no. 5, pp. 885-895, 1991.

[175] S. Whitesall, J. Hoff, A. Vollmer, and L. D'Alecy, “Comparison of simultaneous measurement of mouse systolic arterial blood pressure by radiotelemetry and tail-cuff methods," American Journal of Physiology-Heart and Circulatory Physiology, vol. 286, no. 6, pp. H2408-H2415, 2004.

[176] E. Martel, B. Egner, S. A. Brown, J. N. King, A. Laveissiere, P. Champeroux, and S. Richard, "Comparison of high-definition oscillometry-a non-invasive technology for arterial blood pressure measurement-with a direct invasive method using radiotelemetry in awake healthy cats," Journal of feline medicine and surgery, vol. 15, no. 12, pp. 1104-1113, 2013.

[177] J. Tünsmeyer, K. Hopster, K. Feige, and S. B. Kästner, "Agreement of high definition oscillometry with direct arterial blood pressure measurement at different blood pressure ranges in horses under general anaesthesia," Veterinary anaesthesia and analgesia, vol. 42, no. 3, pp. 286-291, 2015.

[178] H. Stegall, M. Kardon, and W. Kemmerer, "Indirect measurment of arterial blood pressure by doppler ultrasonic sphygmomanometry," Journal of applied physiology, vol. 25, no. 6, pp. 793-798, 1968.

[179] T.-Y. Hsiang, Y.-H. Lien, and H.-P. Huang, "Indirect measurement of systemic blood pressure in conscious dogs in a clinical setting," Journal of Veterinary Medical Science, vol. 70, no. 5, pp. 449-453, 2008.

[180] C. F. Babbs, "Oscillometric measurement of systolic and diastolic blood pressures validated in a physiologic mathematical model," Biomedical engineering online, vol. 11, no. 1, p. 1, 2012. 
[181] L. Geddes, M. Voelz, C. Combs, D. Reiner, and C. Babbs, "Characterization of the oscillometric method for measuring indirect blood pressure," Annals of Biomedical Engineering, vol. 10, no. 6, pp. 271-280, 1982.

[182] A. Bodey, A. Michell, K. Bovee, C. Buranakurl, and T. Garg, "Comparison of direct and indirect (oscillometric) measurements of arterial blood pressure in conscious dogs," Research in veterinary science, vol. 61, no. 1, pp. 17-21, 1996.

[183] K. M. Pedersen, M. A. Butler, A. K. Ersbøll, and H. D. Pedersen, "Evaluation of an oscillometric blood pressure monitor for use in anesthetized cats," Journal of the American Veterinary Medical Association, vol. 221, no. 5, pp. 646-650, 2002.

[184] G. A. Van Montfrans, "Oscillometric blood pressure measurement: progress and problems," Blood pressure monitoring, vol. 6, no. 6, pp. 287-290, 2001.

[185] R. L. Stepien, G. S. Rapoport, R. A. Henik, L. Wenholz, and C. B. Thomas, "Comparative diagnostic test characteristics of oscillometric and doppler ultrasonographic methods in the detection of systolic hypertension in dogs," Journal of veterinary internal medicine, vol. 17, no. 1, pp. 65-72, 2003.

[186] K. M. Meurs, M. W. Miller, and M. R. Slater, "Comparison of the indirect oscillometric and direct arterial methods for blood pressure measurements in anesthetized dogs." Journal of the American Animal Hospital Association, vol. 32, no. 6, pp. 471-475, 1995.

[187] A.-M. Betat, J.-G. Napoleoni, G. Froget, and A. Simonnard, "Non-invasive blood pressure monitoring in ambulatory beagle dogs," Journal of Pharmacological and Toxicological Methods, vol. 62, no. 2, pp. e42-e43, 2010.

[188] A. Z. Mitchell, C. McMahon, T. W. Beck, and R. D. Sarazan, "Sensitivity of two noninvasive blood pressure measurement techniques compared to telemetry in cynomolgus monkeys and beagle dogs," Journal of pharmacological and toxicological methods, vol. 62, no. 1, pp. 54-63, 2010. 
[189] B. Schmelting, M. Niehoff, B. Egner, S. Korte, and G. Weinbauer, "High definition oscillometry: a novel technique for non-invasive blood pressure monitoring in the cynomolgus monkey (macaca fascicularis)," Journal of medical primatology, vol. 38, no. 5, pp. 293-301, 2009.

[190] O. Meyer, R. Jenni, A. Greiter-Wilke, A. Breidenbach, and H. H. Holzgrefe, "Comparison of telemetry and high-definition oscillometry for blood pressure measurements in conscious dogs: effects of torcetrapib," Journal of the American Association for Laboratory Animal Science, vol. 49, no. 4, pp. 464-471, 2010.

[191] X. Zhao, D. Ho, S. Gao, C. Hong, D. E. Vatner, and S. F. Vatner, "Arterial pressure monitoring in mice," Current protocols in mouse biology, pp. 105-122, 2011.

[192] N. J. Cleven, J. A. Müntjes, H. Fassbender, U. Urban, M. Görtz, H. Vogt, M. Gräfe, T. Göttsche, T. Penzkofer, T. Schmitz-Rode, et al., "A novel fully implantable wireless sensor system for monitoring hypertension patients," IEEE Transactions on Biomedical Engineering, vol. 59, no. 11, pp. 3124-3130, 2012.

[193] R. Van Citters and D. Franklin, "Telemetry of blood pressure in free-ranging animals via an intravascular gauge.” Journal of applied physiology, vol. 21, no. 5, pp. 1633-1636, 1966.

[194] T. Shinozaki, R. S. Deane, and J. E. Mazuzan, "The dynamic responses of liquid-filled catheter systems for direct measurements of blood pressure.” Anesthesiology, vol. 53, no. 6, pp. 498-504, 1980.

[195] A. K. Reddy, G. E. Taffet, S. Madala, L. H. Michael, M. L. Entman, and C. J. Hartley, "Noninvasive blood pressure measurement in mice using pulsed doppler ultrasound," Ultrasound in medicine $\mathcal{E}$ biology, vol. 29, no. 3, pp. 379-385, 2003.

[196] T. S. Inc., “Rodent pressure catheters,” Transonic Scisense Inc., Tech. Rep., 2016. [Online]. Available: http://www.transonic.com/product/rodent-pressure-catheters/ 
[197] S. Chatzandroulis, D. Tsoukalas, and P. Neukomm, "A miniature pressure system with a capacitive sensor and a passive telemetry link for use in implantable applications," Microelectromechanical Systems, Journal of, vol. 9, no. 1, pp. 18-23, 2000.

[198] J. Potkay, "Long term, implantable blood pressure monitoring systems," Biomedical microdevices, vol. 10, no. 3, pp. 379-392, 2008.

[199] T. Bowdle et al., "Complications of invasive monitoring." Anesthesiology Clinics of North America, vol. 20, no. 3, p. 571, 2002.

[200] B. Scheer, A. Perel, and U. Pfeiffer, "Clinical review: complications and risk factors of peripheral arterial catheters used for haemodynamic monitoring in anaesthesia and intensive care medicine," Critical Care, vol. 6, no. 3, p. 199, 2002.

[201] E. Frezza, H. Mezghebe, et al., "Indications and complications of arterial catheter use in surgical or medical intensive care units: analysis of 4932 patients." The American Surgeon, vol. 64, no. 2, p. 127, 1998.

[202] R. Wilkins, "Radial artery cannulation and ishaemic damage: a review," Anaesthesia, vol. 40, no. 9, pp. 896-899, 2007.

[203] M. I. Inc., "Millar pressure,” Millar Instruments Inc., Tech. Rep., 2016. [Online]. Available: http://millar.com/products/research/pressure

[204] T. B. Fryer, H. Sandler, S. Freund, E. P. McCutcheon, and E. L. Carlson, "A multichannel implantable telemetry system for flow, pressure, and ecg measurements," Journal of applied physiology, vol. 39, no. 2, pp. 318-326, 1975.

[205] A. M. Leung, W. H. Ko, T. M. Spear, and J. A. Bettice, "Intracranial pressure telemetry system using semicustom integrated circuits," IEEE transactions on biomedical engineering, no. 4, pp. 386-395, 1986. 
[206] S. Gschwend, J. Knutti, H. Allen, and J. Meindl, “A general-purpose implantable multichannel telemetry system for physiological research." Biotelemetry and patient monitoring, vol. 6, no. 3, pp. 107-117, 1978.

[207] R. Armentano, E. Cabrera-Fischer, G. Breitbart, R. Pichel, J. Levenson, and N. Chau, "Telemetry of aortic pressure in unrestrained animals: validation of the method over a wide range of blood pressure (from 40 to $200 \mathrm{mmhg}$ )." Medical progress through technology, vol. 16, no. 3, pp. 125-129, 1989.

[208] P. Valdastri, A. Menciassi, A. Arena, C. Caccamo, and P. Dario, "An implantable telemetry platform system for in vivo monitoring of physiological parameters," IEEE Transactions on Information Technology in Biomedicine, vol. 8, no. 3, pp. 271-278, 2004.

[209] C. McMahon, A. Z. Mitchell, J. L. Klein, A. C. Jenkins, and R. D. Sarazan, "Evaluation of blood pressure measurement using a miniature blood pressure transmitter with jacketed external telemetry in cynomolgus monkeys," Journal of pharmacological and toxicological methods, vol. 62, no. 2, pp. 127-135, 2010.

[210] A. Mitchell, "Continuous blood pressure measurement in conscious, unrestrained monkeys using an implanted mouse transmitter," Journal of Pharmacological and Toxicological Methods, vol. 60, no. 2, p. 257, 2009.

[211] M. Niehoff, J. Sternberg, B. Niggemann, R. Sarazan, and M. Holbrook, "Pimobendan, etilefrine, moxifloxacine and esketamine as reference compounds to validate the dsi physiotel $®$ system in cynomolgus monkeys," Journal of Pharmacological and Toxicological Methods, vol. 70, p. 338, 2014.

[212] M. I. Inc., “Mouse biopotential telemeter,” Millar Intruments Inc., Tech. Rep., 2016. [Online]. Available: http://millar.com/research/products/mouse-telemetry-system/ biopotential-telemeter 
[213] —-, "Telemetry system rat telemeters," Telemetry System Rat Telemeters, Tech. Rep., 2016. [Online]. Available: http://millar.com/research/products/ implantable-rat-telemetry-system

[214] D. S. I. (DSI), "Dsi implantable telemetry," Data Science International (DSI), Tech. Rep., 2016. [Online]. Available: http://www.datasci.com/products/ implantable-telemetry

[215] O. H. Murphy, M. R. Bahmanyar, A. Borghi, C. N. McLeod, M. Navaratnarajah, M. H. Yacoub, and C. Toumazou, "Continuous in vivo blood pressure measurements using a fully implantable wireless saw sensor," Biomedical microdevices, vol. 15, no. 5, pp. 737-749, 2013.

[216] H. J. Knot and D. Lee, "A novel freely moving animal based blood pressure, ecg, and dual body temperature telemetry system for group housed mice in social context," The FASEB Journal, vol. 30, no. 1 Supplement, pp. lb595-1b595, 2016.

[217] H. Fassbender, W. Mokwa, M. Gortz, K. Trieu, U. Urban, T. Schmitz-Rode, T. Gottsche, and P. Osypka, "Fully implantable blood pressure sensor for hypertonic patients," in Sensors, 2008 IEEE. IEEE, 2008, pp. 1226-1229.

[218] J. Fiala, P. Bingger, D. Ruh, K. Foerster, C. Heilmann, F. Beyersdorf, H. Zappe, and A. Seifert, "An implantable optical blood pressure sensor based on pulse transit time," Biomedical microdevices, vol. 15, no. 1, pp. 73-81, 2013.

[219] L. Y. Chen, B. C.-K. Tee, A. L. Chortos, G. Schwartz, V. Tse, D. J. Lipomi, H.-S. P. Wong, M. V. McConnell, and Z. Bao, "Continuous wireless pressure monitoring and mapping with ultra-small passive sensors for health monitoring and critical care," Nature communications, vol. 5, 2014.

[220] R. Pais, A. Duttaroy, M. Dobbs, and E. Pastalkova, "Implantable blood pressure monitoring cuff for small laboratory animal," $N A, 2010$. 
[221] C. Wu, T. Skalak, T. Schwenk, C. Mahler, A. Anne, P. Finnerty, H. Haber, R. Weikle, M. Feldman, et al., "Accuracy of the conductance catheter for measurement of ventricular volumes seen clinically: effects of electric field homogeneity and parallel conductance," Biomedical Engineering, IEEE Transactions on, vol. 44, no. 4, pp. 266-277, 1997.

[222] E. Lankford, D. Kass, W. Maughan, and A. Shoukas, "Does volume catheter parallel conductance vary during a cardiac cycle?" American Journal of Physiology-Heart and Circulatory Physiology, vol. 258, no. 6, pp. H1933-H1942, 1990.

[223] D. Georgakopoulos, W. Mitzner, C. Chen, B. Byrne, H. Millar, J. Hare, and D. Kass, "In vivo murine left ventricular pressure-volume relations by miniaturized conductance micromanometry," American Journal of Physiology-Heart and Circulatory Physiology, vol. 274, no. 4, pp. H1416-H1422, 1998.

[224] J. Nielsen, S. Kristiansen, S. Ringgaard, T. Nielsen, A. Flyvbjerg, A. Redington, and H. Bøtker, "Left ventricular volume measurement in mice by conductance catheter: evaluation and optimization of calibration," American Journal of Physiology-Heart and Circulatory Physiology, vol. 293, no. 1, pp. H534-H540, 2007.

[225] P. White, C. Brookes, H. Ravn, E. Stenbøg, T. Christensen, R. Chaturvedi, K. Sorensen, V. Hjortdal, and A. Redington, "The effect of changing excitation frequency on parallel conductance in different sized hearts," Cardiovascular research, vol. 38, no. 3, pp. 668$675,1998$.

[226] T. J. Gawne, K. S. Gray, and R. E. Goldstein, "Estimating left ventricular offset volume using dual-frequency conductance catheters," Journal of Applied Physiology, vol. 63, no. 2, pp. 872-876, 1987. [Online]. Available: http: //jap.physiology.org/content/63/2/872.abstract 
[227] D. Georgakopoulos and D. Kass, "Estimation of parallel conductance by dual-frequency conductance catheter in mice," American Journal of Physiology-Heart and Circulatory Physiology, vol. 279, no. 1, pp. H443-H450, 2000.

[228] C. Wei, J. Valvano, M. Feldman, and J. Pearce, "Nonlinear conductance-volume relationship for murine conductance catheter measurement system," Biomedical Engineering, IEEE Transactions on, vol. 52, no. 10, pp. 1654-1661, 2005.

[229] C. Wei, J. Valvano, M. Feldman, M. Nahrendorf, R. Peshock, and J. Pearce, "Volume catheter parallel conductance varies between end-systole and end-diastole," Biomedical Engineering, IEEE Transactions on, vol. 54, no. 8, pp. 1480-1489, 2007.

[230] J. Porterfield, A. Kottam, K. Raghavan, D. Escobedo, J. Jenkins, E. Larson, R. Treviño, J. Valvano, J. Pearce, and M. Feldman, "Dynamic correction for parallel conductance, gp, and gain factor, $\alpha$, in invasive murine left ventricular volume measurements," Journal of Applied Physiology, vol. 107, no. 6, pp. 1693-1703, 2009.

[231] K. Raghavan, J. Porterfield, A. Kottam, M. Feldman, D. Escobedo, J. Valvano, and J. Pearce, "Electrical conductivity and permittivity of murine myocardium," Biomedical Engineering, IEEE Transactions on, vol. 56, no. 8, pp. 2044-2053, 2009.

[232] M. Reyes, M. Steinhelper, J. Alvarez, D. Escobedo, J. Pearce, J. Valvano, B. Pollock, C. Wei, A. Kottam, D. Altman, et al., "Impact of physiological variables and genetic background on myocardial frequency-resistivity relations in the intact beating murine heart," American Journal of Physiology-Heart and Circulatory Physiology, vol. 291, no. 4, pp. H1659-H1669, 2006.

[233] M. I. Inc., “Complete pv loop analysis,” Millar Instruments Inc., Tech. Rep., 2016. [Online]. Available: http://millar.com/products/research/pressure-volume

[234] K. Le, "High performance wireless bio-impedance measurement system," Master's thesis, The University of Texas at Austin, 2014. 
[235] S. Popa, K. Fricke, J. Dubois, A. Kottam, and R. Sobot, "Murine heart volume: Numerical comparison and calibration of conductance catheter models," Biomedical Engineering, IEEE Transactions on, vol. 61, no. 9, pp. 2396-2405, 2014.

[236] G. P. van Hout, S. J. Jansen, J. M. Gho, P. A. Doevendans, W. W. van Solinge, G. Pasterkamp, S. A. Chamuleau, and I. E. Hoefer, “Admittance-based pressure-volume loops versus gold standard cardiac magnetic resonance imaging in a porcine model of myocardial infarction," Physiological reports, vol. 2, no. 4, p. e00287, 2014.

[237] J. Porterfield and J. Pearce, "Comparison of conductance to volume equations: The gain coefficient $\alpha$," in Engineering in Medicine and Biology Society, 2009. EMBC 2009. Annual International Conference of the IEEE. IEEE, 2009, pp. 3043-3046.

[238] C. L. Wei and M. H. Shih, "Calibration Capacity of the Conductance-to-Volume Conversion Equations for the Mouse Conductance Catheter Measurement System," IEEE Transactions Biomedical Engineering, vol. 56, no. 6, pp. 1627-1634, 2009.

[239] K. Fricke, A. Dounavis, and R. Sobot, "Wireless telemetry system for implantable cardiac monitoring in small animal subjects using pressure-volume sensors," in IEEE 11th International NEWCAS 2013 Conference, June 16-19 2013, Paris, France, 2013.

[240] K. Fricke, F. Konecny, A. El-Warrak, C. Hodgson, H. Cadieux-Pitre, T. Hill, and R. Sobot, "In-vivo characterization of left-ventricle pressure-volume telemetry system in swine model," Biomedical microdevices, vol. 18, no. 5, p. 75, 2016.

[241] T. Instruments, MSP430X22X4 Mixed Signal Microcontroller, SLAS504D, July 2006.

[242] — MSP430X2XX Family User's Guide, SLAU144e, 2008.

[243] N. K. Nithya Subramanian, Anaren 0404 balun optimized for TI CC2500 Transceiver, Anaren, April 2008. 
[244] T. Electronics, UMCC - Ultraminiatrue Coax Connector \& Cable Assembly Series, 2007.

[245] Molex, Molex SlimStack 0.4mm Pitch Board-to-Board Connectors, July 2012.

[246] GE, GE P161 3F Gauge Pressure Sensor Die, General Electric, 2012.

[247] S. R. Systems, About Lock-In Amplfiers-Application Note 3.

[248] J. Scofield, "Frequency-domain description of a lock-in amplifier," American Journal of Physics, vol. 62, no. 2, pp. 129-132, 1994.

[249] M. Meade, "Lock-in amplifiers: principles and applications," Majalah LAPAN, vol. 1, 1983.

[250] A. De Marcellis, G. Ferri, A. D’Amico, C. Di Natale, and E. Martinelli, “A fully-analog lock-in amplifier with automatic phase alignment for accurate measurements of ppb gas concentrations," Sensors Journal, IEEE, vol. 12, no. 5, pp. 1377-1383, 2012.

[251] M. Gabal, N. Medrano, B. Calvo, and S. Celma, "A low-voltage single-supply analog lock-in amplifier for wireless embedded applications," in Smart Objects: Systems, Technologies and Applications (RFID Sys Tech), 2010 European Workshop on. VDE, 2010, pp. 1-6.

[252] J. Aguirre, N. Medrano, B. Calvo, and S. Celma, "Lock-in amplifier for portable sensing systems," Electronics letters, vol. 47, no. 21, pp. 1172-1173, 2011.

[253] G. de Graaf and R. Wolffenbuttel, "Lock-in amplifier techniques for low-frequency modulated sensor applications," in Instrumentation and Measurement Technology Conference (I2MTC), 2012 IEEE International. IEEE, 2012, pp. 1745-1749.

[254] R. Mancini, Op amps for everyone. Newnes, 2003.

[255] Molex, 0.50mm Pitch SMT Right-Angle FFC/FPC Connector, March 2012. 
[256] F. T. D. I. Ltd., "Ftdi chip drivers,” Future Technology Devices International Ltd., Tech. Rep., 2016. [Online]. Available: http://www.ftdichip.com/FTDrivers.htm

[257] K. Fricke, Z. Wang, and R. Sobot, "In-vitro rf characterization of implantable telemetry system," Analog Integrated Circuits and Signal Processing, vol. 81, no. 3, pp. 635-644, 2014.

[258] K. Fricke, R. Sobot, and C. Hodgson, "Design and evaluation of a 3d printed biocompatible capsule for implantable telemetry systems," in Biomedical Circuits and Systems Conference (BioCAS), 2014 IEEE. IEEE, 2014, pp. 368-371.

[259] E. Chow, M. Morris, and P. Irazoqui, "Implantable rf medical devices: The benefits of high-speed communication and much greater communication distances in biomedical applications," Microwave Magazine, IEEE, vol. 14, no. 4, pp. 64-73, June 2013.

[260] A. Poon, S. O’Driscoll, and T. Meng, “Optimal frequency for wireless power transmission into dispersive tissue," Antennas and Propagation, IEEE Transactions on, vol. 58, no. 5, pp. 1739-1750, May 2010.

[261] E. Y. Chow, Y. Ouyang, B. Beier, W. J. Chappell, and P. P. Irazoqui, "Evaluation of cardiovascular stents as antennas for implantable wireless applications," Microwave Theory and Techniques, IEEE Transactions on, vol. 57, no. 10, pp. 2523-2532, 2009.

[262] U.-M. Jow and M. Ghovanloo, "Modeling and optimization of printed spiral coils in air, saline, and muscle tissue environments," IEEE transactions on biomedical circuits and systems, vol. 3, no. 5, pp. 339-347, 2009.

[263] S. Mirbozorgi, B. Gosselin, and M. Sawan, "A transcutaneous power transfer interface based on a multicoil inductive link," in 2012 Annual International Conference of the IEEE Engineering in Medicine and Biology Society. IEEE, 2012, pp. 1659-1662. 
[264] C. C. Johnson and A. W. Guy, "Nonionizing electromagnetic wave effects in biological materials and systems," Proceedings of the IEEE, vol. 60, no. 6, pp. 692-718, 1972.

[265] K. Bordjih, J.-Y. Jouzeau, D. Mainard, E. Payan, J.-P. Delagoutte, and P. Netter, "Evaluation of the effect of three surface treatments on the biocompatibility of 3161 stainless steel using human differentiated cells," Biomaterials, vol. 17, no. 5, pp. 491-500, 1996.

[266] M. Assad, N. Lemieux, C. Rivard, and L. Yahia, "Comparative in vitro biocompatibility of nickel-titanium, pure nickel, pure titanium, and stainless steel: genotoxicity and atomic absorption evaluation," Bio-medical materials and engineering, vol. 9, no. 1, pp. $1-12,1999$.

[267] ZMDI, “Zmdi zsc 31015 evaluation datasheet," ZMDI, Tech. Rep., 2016. [Online]. Available: http://www.idt.com/document/man/ zsc31010zsc31015zssc3015-zacwire-ssc-modular-evaluation-kit-description

[268] I. W. Labscribe, "Labscribe software,” I Worx Labscribe, Tech. Rep., 2016. [Online]. Available: https://www.iworx.com/research/software/labscribe-software-2/

[269] V. Labs, "Vivitro labs - pulse duplicator," Vivitro Labs, Tech. Rep., 2016. [Online]. Available: http://vivitrolabs.com/product/pulse-duplicator/

[270] K. Fricke, F. Konecny, A. El-Warrak, C. Hodgson, H. Cadieux-Pitre, T. Hill, and R. Sobot, "Real-time telemetric physiologic recordings of ventricle pressure-volume in an awake swine model with histopathological evaluation," in Biomedical Circuits and Systems Conference (BioCAS), 2015 IEEE. IEEE, 2015.

[271] L. Luo, K. Gannes, K. Fricke, S. Senjuti, and R. Sobot, "Low-power cmos voltage regulator architecture for implantable rf circuits," in RFID Technology (EURASIP RFID), 2012 Fourth International EURASIP Workshop on. IEEE, 2012, pp. 99-106. 
[272] M. Cai, K. Fricke, and R. Sobot, "Embedded rf switch for implantable telemetry systems designed in 130nm cmos," in Circuits and Systems (ISCAS), 2016 IEEE International Symposium on. IEEE, 2016, pp. 2735-2738.

[273] N. Modir, K. Fricke, A. Zine-Eddine, and R. Sobot, "Controlling and signal processing core for wireless implantable telemetry system," in Electronics, Circuits and Systems (ICECS), The 23rd IEEE International Conference on. IEEE, 2016.

[274] N. Bako, K. Fricke, R. Sobot, and A. Baric, "A $11 \mu \mathrm{W}$, 9-bit fully differential, cyclic/algorithmic adc in $0.13 \mu \mathrm{m}$ cmos," International Journal of Circuit Theory and Applications, 2016. 


\section{Curriculum Vitae}

Name: $\quad$ Kyle Fricke

Post-Secondary The University of Western Ontario

Education and London, Ontario, Canada

Degrees: $\quad$ PhD - Candidate (2012 - Present)

The University of Western Ontario

London, Ontario, Canada

2010 - 2012 M.E.Sc.

2006 - 2010 B.E.Sc.

Honours and Doctoral Excellence Research Award

Awards: $\quad 2016$

Graduate Student Award for Excellence in Research

2016

Engineering Implants for Better Health - Media publication

http://www.giving.westernu.ca/your-impact/publications/

impact-western/fall-2015/engineering-implants-for.html

2015 
Outstanding Presentation in Graduate Symposium

2012, 2014, 2016

Best Student Paper Award IEEE CCECE 2015

2015

National Science and Engineering Research Council Canada Graduate Scholarship $2015-2017$

Western University USC Teaching Honour Roll - Instructor 2014,2015

Ontario Graduate Scholarship

2012, 2014, 2015

MacKay-Lassonde Scholarship in Computer Engineering 2010

$\begin{array}{ll}\text { Related Work } & \text { Lecturer - ECE 3349A - Introduction to VLSI } \\ \text { Experience: } & \text { The University of Western Ontario } \\ & 2014-2016\end{array}$

Teaching Assistant

The University of Western Ontario

$2010-2016$ 


\section{Publications:}

\section{Refereed Journals}

[1] N. Bako, K. Fricke, A. Baric, R. Sobot, "A $11 \mu \mathrm{W}$, 9-Bit fully differential, cyclic/algorithmic ADC in $0.13 \mu \mathrm{m}$ CMOS, International Journal of Circuit Theory and Applications, Wiley, 2016 [2] K. Fricke, C. Hodgson, F. Konecny, A. El-Warrak, H. Cadieux-Pitre, T. Hill, R. Sobot, "Invivo Characterization of Left-Ventricle Pressure-Volume Telemetry System in Swine Model, Biomedical Microdevices, vol. 18, no. 5, pp 1-17, Springer, 2016

[3] K. Fricke, Z. Wang, R. Sobot, "In-Vitro RF Characterization of Implantable Telemetry System, Analog Integrated Circuits and Signal Processing, vol. 81, no. 3, pp 635-644, Springer, 2014

[4] S. Popa, K. Fricke, J. Dubois, A. Kottom, R. Sobot. "Murine Heart Volume: Numerical Comparison and Calibration of Conductance Catheter Models, Biomedical Engineering, IEEE Transactions on, 2014, DOI: 10.1109/TBME.2014.2313621

[5] K. Fricke, A. Dounavis, R. Sobot, "Analogue Portable Electrooculogram Real-Time Signal Processor, International Journal of Circuit Theory and Applications, Wiley, 2012

\section{Refereed Conference Proceedings}

[1] N. Modir, K. Fricke, Z. Abid, R. Sobot, “Controlling and Signal Processing Core for Wireless Implantable Telemetry System, IEEE International Conference on Electronics Circuits and Systems (ICECS), December 11-14, 2016

[2] M. Cai, K. Fricke, R. Sobot, "Embedded RF Switch for Implantable Telemetry Systems Designed in 130nm CMOS, IEEE ISCAS 2016 Conference, May 22-24, 2016, Montreal, Canada

[3] K. Fricke, C. Hodgson, F. Konecny, A. El-Warrak, Chad Hodgson, H. Cadieux-Pitre, T. Hill, R. Sobot, "Real-Time Telemetric Physiologic Recordings of Ventricle Pressure-Volume and Rate of Contraction in an Awake Swine Model, IEEE BIOCAS 2015 Conference, October 22-24, 2015, Atlanta, USA

[4] K. Fricke, C. Hodgson, F. Konecny, A. El-Warrak, H. Cadieux-Pitre, T. Hill, R. Sobot, "In- 
Vivo Study of Cardiac PressureVolume Curves using Implantable RF Telemetry, 37th Annual International Conference of the IEEE Engineering in Medicine and Biology Society, August 25-29, 2015, Milan, Italy

[5] K. Fricke, M. Cai, R. Sobot, "CMOS Voltage Regulator for RF Energy Harvester, IEEE NEWCAS 2015 Conference, June 7-10, 2015, Grenoble, France

[6] R. Ganta, M. Cai, K. Fricke, R. Sobot, "Design of a 50nW, 0.5VDD Sub-Threshold OTA Amplifier with Indirect Compensation Technique and Class AB Output Stage, CCECE 2015 Conference, May 3-6, 2015, Halifax, Nova Scotia, Canada

[7] M. Cai, K. Fricke, R. Sobot, "Design of a low-power 2.4GHz Current Reuse VCO for Biomedical Implantable Applications, CCECE 2015 Conference, May 3-6, 2015, Halifax, Nova Scotia, Canada

[8] K. Fricke, C. Hodgson, R. Sobot. "Design and Evaluation of a 3D Printed Bio-Compatible Capsule for Implantable Telemetry Systems, IEEE BioCAS 2014 Conference, Oct 22-24 2014, Lausanne, Switzerland

[9] K. Fricke, R. Sobot, "Live Demonstration: Wireless Telemetry System for Implantable Pressure-Volume Cardiac Monitoring, IEEE BioCAS 2014 Conference, Oct 22-24 2014, Lausanne, Switzerland

[10] K. Fricke, R. Sobot, "Miniature Implantable Telemetry System for Pressure-Volume Cardiac Monitoring, IEEE BioCAS 2013 Conference, Oct 31-Nov. 2 2013, Rotterdam, Netherlands

[11] K. Fricke, A. Dounavis, R. Sobot, "Wireless Telemetry System for Implantable Cardiac Monitoring in Small Animal Subjects Using Pressure-Volume Sensors, IEEE 11th International NEWCAS 2013 Conference, June 16-19 2013, Paris, France

[12] L. Luo, K. De Gannes, K. Fricke, S. Senjuti, R. Sobot, "LowPower CMOS Voltage Regulator Architecture for Implantable RF Circuits, The 4th International EURASIP Workshop on RFID Technology, RFID 2012, September 27-28, 2012, Torino, Italy

[13] S. Popa, K. Fricke, R. Sobot, "Calibration and Finite Element Numerical Analysis of an 
Admittance Based Volume Sensor for the Murine Heart, IEEE International Conference for Upcoming Engineers, ICUE 2012, August 2, 2012, Toronto, ON, Canada

[14] S. Senjuti, K. Fricke, A. Dounavis, R. Sobot, "Misalignment Analysis for ResonanceBased Wireless Power Transfer to Biomedical Implants, 2012 IEEE Canadian Conference on Electrical and Computer Engineering, CCECE2012, April 29 - May 2, 2012, Montreal, QC, Canada 